\title{
LA-8213-MS
}

DR. 885

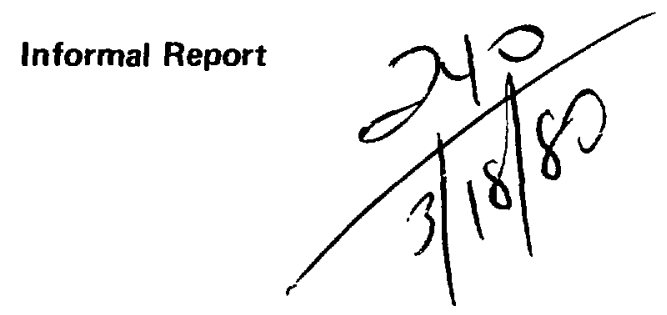

\section{Documentation of TRU Biological Transport Model}

(BIOTRAN)

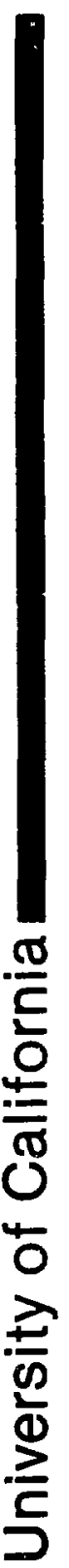

\section{MASTER}

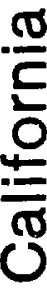




\title{
DOCUMENTATION OF TRU BIOLOGICAL TRANSPORT MODEL (BIOTRAN)
}

\author{
by

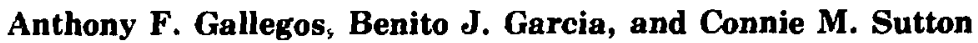

\begin{abstract}
Inclusive of Appendices, this document describes the purpose, rationale, construction, and operation of a biological transport model (BIOTRAN). This model is used to predict the flow of transuranic elements (TRU) through specified plant and animal environments using biomass as a vector. The appendices are:

A) Flows of moisture, biomass, and TRU

B) Intermediate variables affecting flows

C) Mnemonic equivalents (code) for variables

D) Variable library (code)

E) BIOTRAN code (Fortran)

F) Plants simulated

G) BIOTRAN code documentation

H) Operating instructions for BIOTRAN code

The main text is presented with a specific format which uses a minimum of space, yet is adequate for tracking most relationships from their first appearance to their formulation in the code. Because relationships are treated individually in this manner, and rely heavily on Appendix material for understanding, it is advised that the reader familiarize himself with these melterials before proceeding with the main text.
\end{abstract}

\section{INTRODUCTION}

Several documents ${ }^{1,2}$ have been completed which provide and/or describe capabilities, operating instructions, and code documentation of the TRU Biological Transport Model (BIOTRAN) developed for LASL. Previous LASL Progress reports, ${ }^{3,4,6}$ one other LASL report, ${ }^{\circ}$ and LASL presentations ${ }^{7,8,9}$ have provided limited preliminary documentation of model aspects including validation, stability, utilization capability, and sensitivity analysis. This report will provide the rational used in the development and utilization of the model itself. Data obtained from field studies, or other data bases used in validating certain aspects of the mode! will be presented in another report. Hopefully, this report will put into perspective model capabilities, limitations, and usefulness of the model in predicting the movement of TRU and other radionuclides and nutrients through ecosystems using biomass as a vector. 


\section{BACKGROUND ON MODEL DEVELOPMENT AND UTILIZATION}

A generalized description of BIOTRAN is presented in Figure 1 as it was generally conceived for application to a given grid of the stucly area, ${ }^{3}$ and was used as the basis for model development allowing some modification. The model was constrained to permit exchanges and transports of TRU between what were , onsidered the most significant pathways. A modular approach was decided upon for realization of transport processes as indicated, however, some modifications, deletions, and conso!idation of modules became necessary or more expedient as model development proceeded." All of the major components (Atmosphere, Lithosphere, Plants-ie) were included as part of the present model with exception of (MAN) which was left for possible future interphasing vith modules of this type. ${ }^{10}$ 'The aquatic module of Hydrosphere was never initiated due to time constraints and other priorities. Other portions of Hydrosphere including transport of TRU through runoff and soil erosior were aiso not considered. The modeling efforts on Atmosphere ${ }^{3,4}$ with respect to predicting TRU air concentrations over a given grid of the study area were not included as part of the model in lieu of providing "scenario" capability for both chronic and acute TRU releases.'

Efforts were also directed toward formulating inter-regional (grid) transport routes for TRU as described when an overall status report of module development was presented (Fig. 2); ${ }^{4}$ although inter-grid transport is not part of the present model, the drscription does indicate how the original conception of the model was changing to meet pivject needs, and can be used to compare with the final conceptual version of the model (Fig. 3). Hence, the present model provides ouiput for modeling TRU transport to humans, but does not include the latter as such. In addition, other needs (modules) required for $m$ del operation, and module "lumping" into subroutines for codification has complicated the relationship between model components and coding arrangement as shown in Fig. 4. ${ }^{2}$ The main program (PLANTS) carries all elements previously included in Lithosphere, Hydrosphere, Atmosphere, and most elements of Plants of the earliest model. ${ }^{3}$ The Plant component may require as many as three subroutines, depending on user options, ${ }^{1}$ while all elements for Animal are located in one subroutine. ${ }^{1}$ For this reason must modules developed are not separate entities, but are imbedded within larger groupings as submodules with varying degrees of dependence on each other for proper operation as will be shown later in this report.

BIOTRAN was constr'deted primarily to fulfill several project objectives: ${ }^{3}$

1. to model the moverient of Transuranics (TRU) through ecosystems within the study area.

2. to provide enough ficuerality for extending its use to other areas.

3. to be capable of predicting both short and long range cycling of TRU in a number of climatic regimes.

4. to provide output which could be used in evaluating potential hazards to man from Transuranics (TRU) as a result of waste storage and accidental relcases.

Early in the modeling phase it was decided to model concomitantly:

1. flow of biomass

2. flow of water

3. flow of TRU

and to construct a mathematical model of intermediate complexity consisting primarily of:

1. State variables-model elements that comprise the system structure (compartments).

2. Driving variables-model elements which vary with time, affect flow of pool material, but are largely independent of the system.

3. Intermediate variables-model elements which control or affect flow of pool material from one state (compartment) to another. 


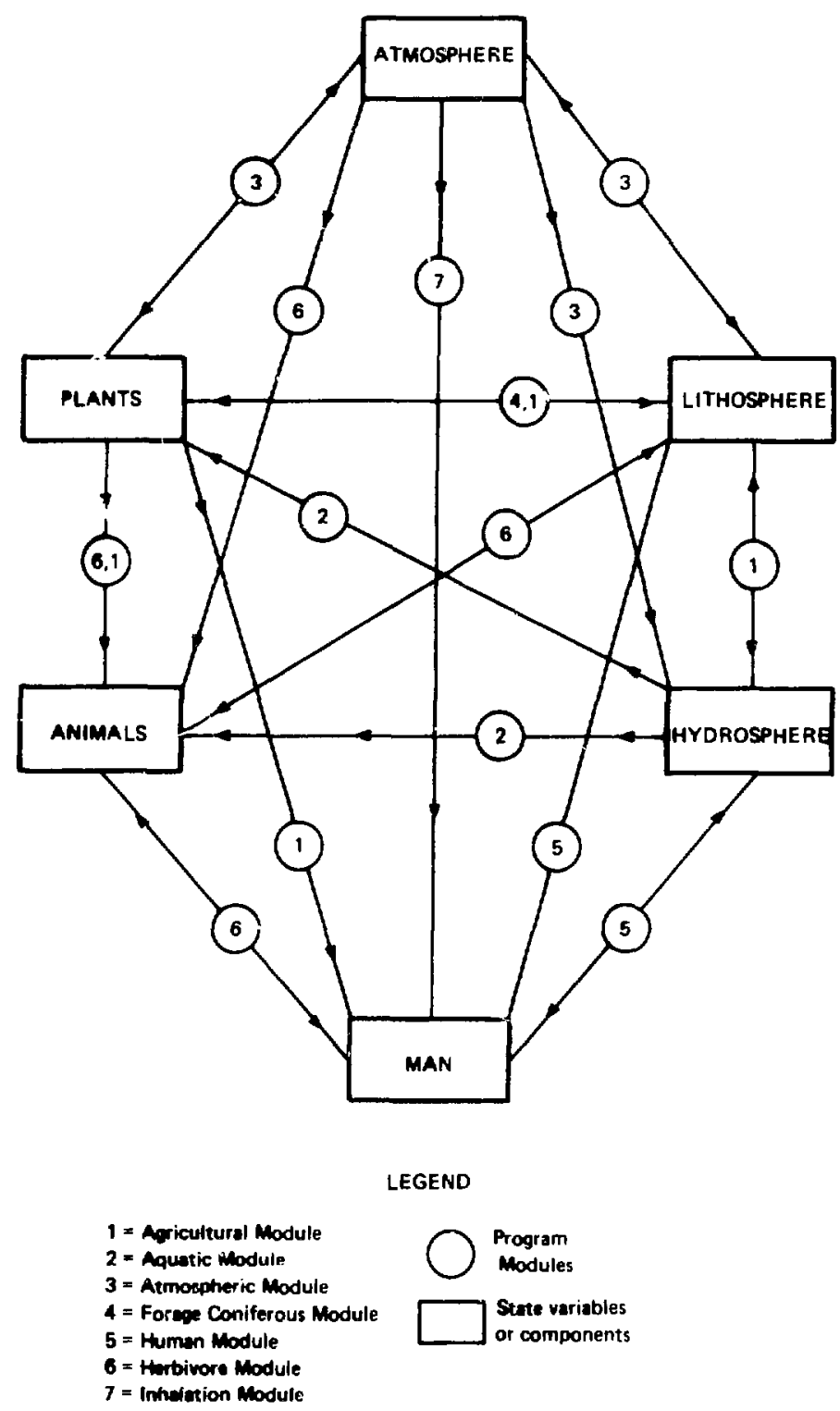

Fig. 1.

TRU biological transport model.

Furthermore, it was decided that the flow structure of the model would best be represented mathematically as a set of difference equations based on daily iterations which would govern input and output of pool material within and among states involving all three types of variables mentioned. An illustration of such a strategy was reported on earlier, and illustrated (Fig. 5) for forage plants ${ }^{3}$ where the identification of principal flow effectors was described and given preliminary documentation. Subsequent development has followed this strategy as will be shown in the next section of this report; however, generalization, refinement, and sensitivity techniques were extended to all portions of the model at all times during the course of development. 

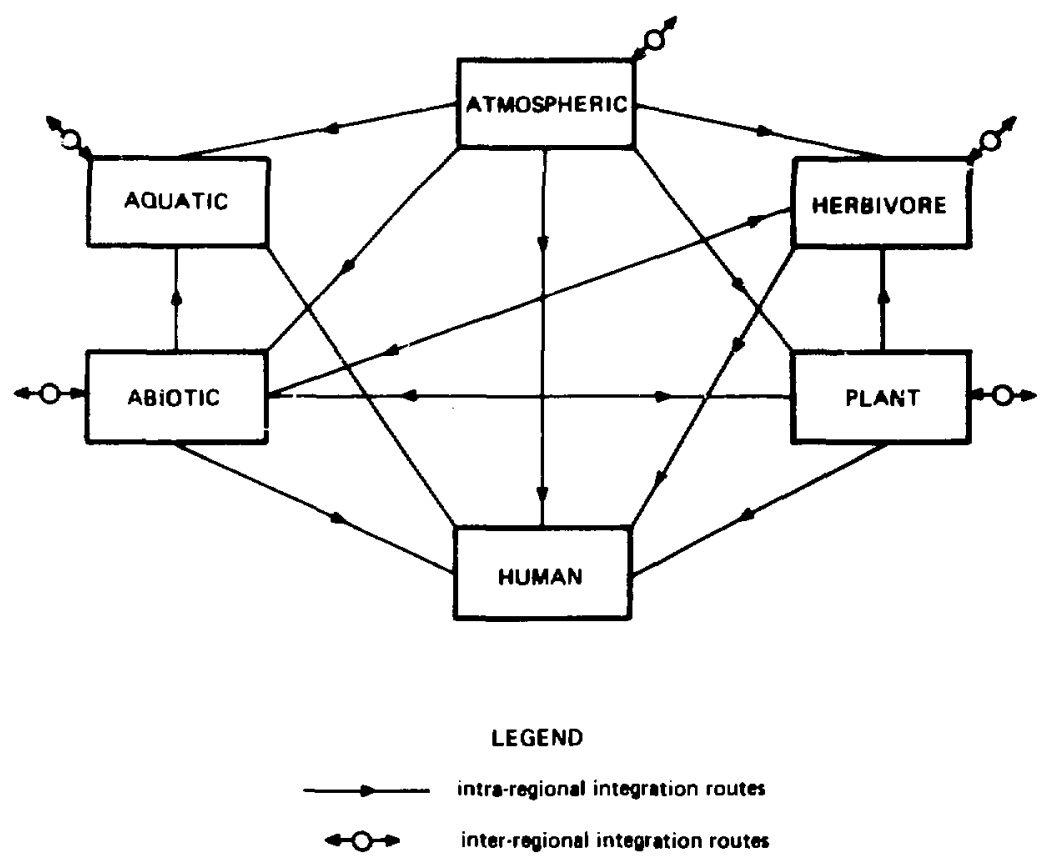

Fig. 2.

Intraregional and interregional integration routes of TRU.

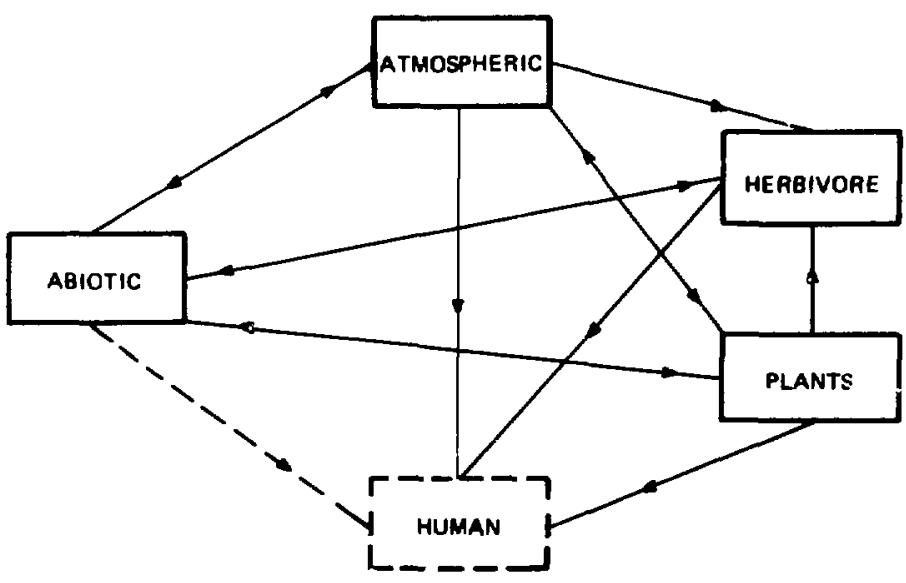

LEGEND

modeled transports and components

- _ - hypothetical or potential trensports and components

Fig. 3.

Present status of model development for single grid TRU transport. 


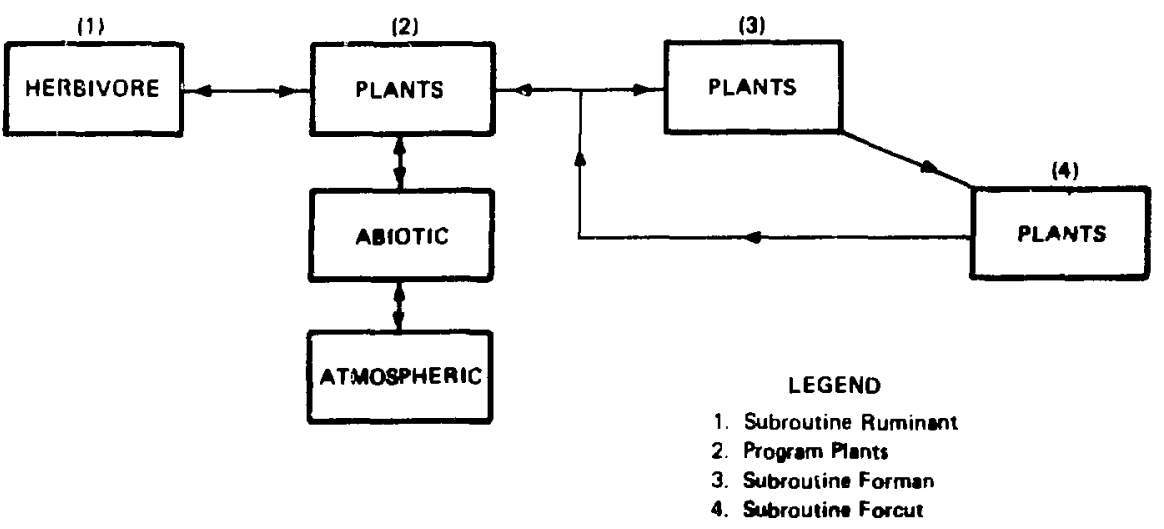

Fig. 4.

Relationship between model components and model code.

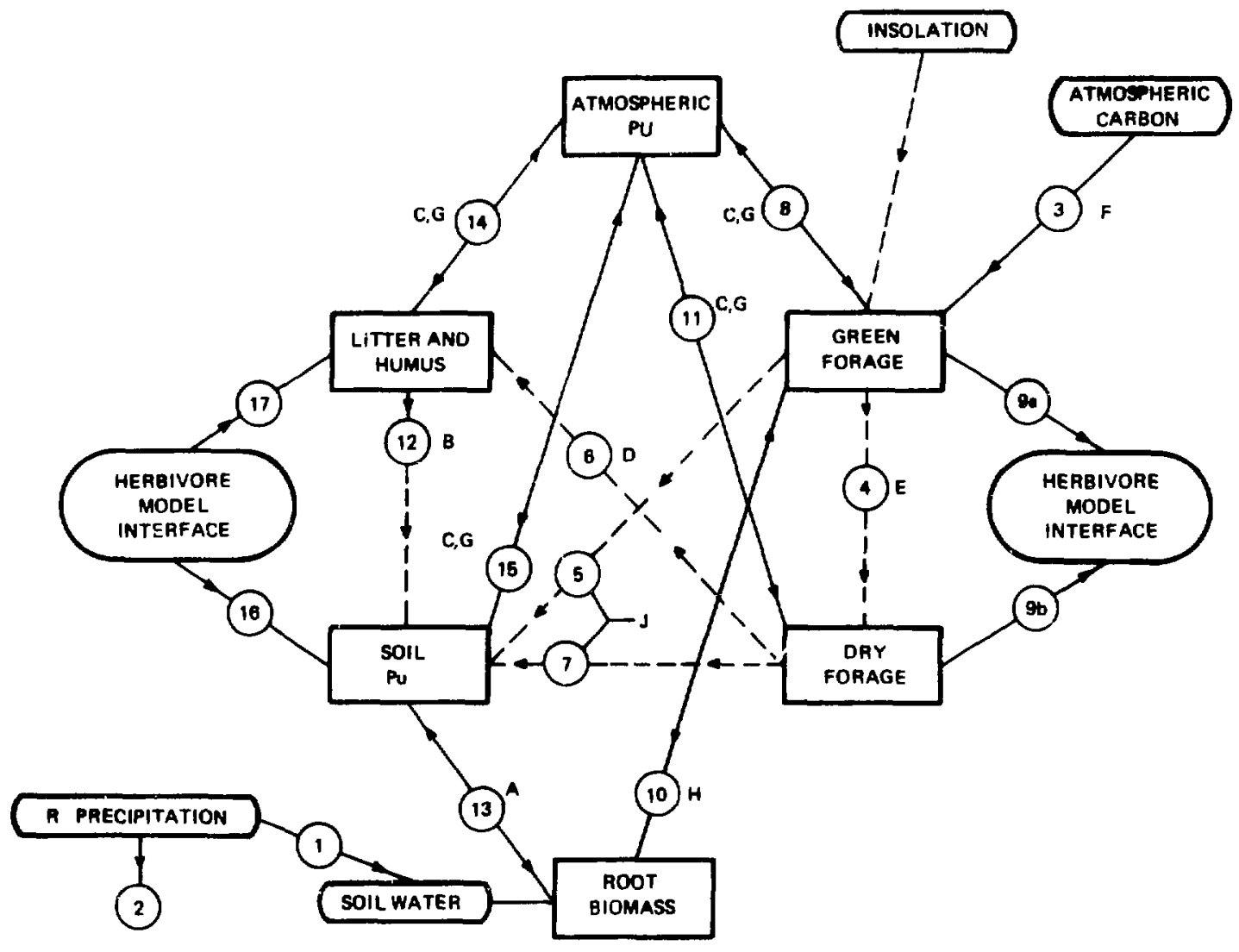

Fig. 5.

Letters indicate physiochemical and biological processes. Numbers indicate transport rate; processes are listed in accompanying table. 
Transport Rate
No.

1

2

3

4

5

6

7

8

$9 a, b$

10

11

12

13

14

15

16-18
Description of Process

Precipitation to soil storage

Surface run-off from soil

Biomass production from

photosynthesis

Death of live above ground biomass

Loss of Pu from above ground biomass (surface)

Production of litter and humus from standing dead

Loss of Pu from above ground dead biomass (surface)

Loss of $\mathrm{Pu}$ from above ground standing biomass (surface), and deposition from atmosphere

Loss of above ground standing biomass to grazers

Exchange of Pu and biomass between roots and above ground standing biomass

Loss of Pu from above ground dead standing biomass, and deposition from atmosphere

Transport of Pu to soil from litter and humus

Transport of $\mathrm{Pu}$ from soil to roots and roots to soil

Exchange of Pu between litter and humus (surface)

Exchange of Pu between soil and atmosphere

Grazing model interfacing
Controlling Variables Used in Determining the Specific Transport Rate

Precipitation regulation, soil water

Soil water, temperat ure, live above ground biomass

Soil water, live above ground biomiss, temperature, insolation

Soil water, live above ground biomass. temperature

Precipitation regulstion, time

Above ground dead st anding biomass, temperature, soil water precipitation regulation, above ground st anding biomass

Live ahove ground hiomass, atmospheric $\mathrm{Pu}$. cime, precipitation regulation

Description in grazing module

Live above ground biomass, root biomass. temperature

Dead above ground standing biomass, atmospheric $\mathrm{Pu}$, time, precipitation regulation

Litter and humus, temperature, soil water

Root biomass, soil Pu, temperature (root death)

Litter and humus. al mospheric $\mathrm{Pu}$, precipitation regulation time

Soil $\mathrm{Pu}$, atmospheric $\mathrm{Pu}$, precipitation regulation, time

Description of variables in grazing model

a'Time enters as variable as a removal decay constant based on dry deposition or resuspension of $\mathrm{Pu}$. 


\section{STRATEGY USED FOR MODEL ANALYSIS}

This document is written with the view that there is not a single analysis strategy which can be used to document a model. The work of IBP investigators ${ }^{11}$ in this regard suggests a mathematical approach allured to earlier which identifies flows, and catalogues variables of different types that affect transport mechanisms. However, not all model structure is easily amenable to such a strategy, and may even lead to confusion when operations other than flow are integral parts of a model such as this one. Yet a strict analysis of relationships utilized in a model without emphasis on system construction also may fall short of putting into perspective interrelationships between component parts. Hence, both types of strategies have been adopted for this report although the mathematical approach has been modified to integrate the two strategies more closely. Box-and-arrow diagrams indicating component arrangements will be decomposed into small, more manageable portions as necessary, rather than presenting the total model all at once. Certain conventions will be adopted; compartments (state variables) will be identified $\left(X_{1}, i=1, n\right)$ for a given pool $(P)$; the units of flow will be specified. Flow diagrams will be presenied such as:

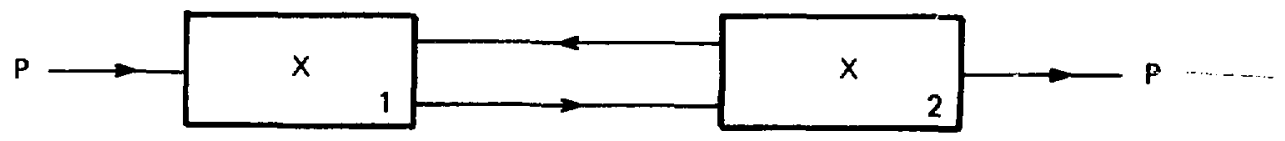

where

$\left(X_{1}\right)=$ names for a specific compartment (system)

$(1,2)=$ compartment index: $X_{1}, X_{2}$

$\mathrm{P} \quad=$ gain or loss of exchanged material frum the system

Several types of flows can be constructed from this example:

$F(P, 1)=$ transport into $X_{1}$ from outside system (input)

$F(1,2)=$ transport from $X_{1}$ to $X_{2}$

$F(2,1)=$ transport from $X_{2}$ to $X_{1}$

$\mathrm{F}(2, \mathrm{P})=$ transport from $\mathrm{X}_{2}$ to outside system (sink)

Factors affecting flow can be indicated in functional form such as:

$F(I, K)=f\left(X_{1}, I_{j}, D_{k}\right)$

where

$\mathrm{X}_{1}=$ ith state variable

$I_{\mathrm{J}}=\mathrm{jth}$ intermediate variable

$D_{k}=D_{k}$ driving variable 
These variables will be identified, catalogued, and related to the model code variable library (Appendix D), and code documentation (Appendix $\mathrm{H}$ ). In addition, justification for the use of any or all factors affecting a flow of pooled material will be presented as required throughout the analysis. The other variables or their mnemonic equivalents:

$\mathrm{C}_{1}=$ Lth constant

$\mathbf{O}_{\mathbf{m}}=\mathrm{mth}$ option or user decisions variable

$\mathrm{L}_{\mathrm{n}}=\mathrm{nth}$ logic or boundary variable

$S_{0}=$ oth stochastic variable,

will complete the set of variables required for model documentation and are present in Appendix C. They include the location address in the code. Finally, ins antaneous transport or exchange between compartments is represented as:

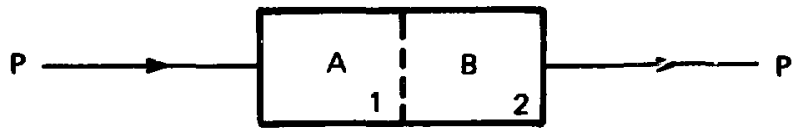

where the vertical dashed line indicates this type of transport, and $F(1 \cdot 2)$ in.stead of $F(1,2)$ is used to represent the function for transport $0^{\text {: }}$ material from $X_{1}$ to $X_{2}$.. ie. Other conventions used will be discussed as they arise. Mnemonic symbols will not be ried in describing the flows as they are considered in this fashion in the code documentatic.. (Appendix $G$ ).

The analysis will be performed first by identifying flows of pool material, and proceed to document the relationships as required. Water, biomass, and TRU flows will be considered in that order although parallel relationships between flows will be establisheci as they occur; the order of documentation will follow as closely as possible that established in the code document (Appendix G). Variables which are functions of other variables (secondary variables) will be developed within the text as necessery.

The appendices included in this document are as follows:

Appendix A. Flows

Appendix B. Equations Involving Secondary Intermediate Variables

Aprendix C. Variables and Their Mnemonic Equivalents

Appendix D. Variable Library

Appendix E. TRU Biological Transport Model Code (BIOTRAN)

Appenclix F. Plant Number Positions

Appendix G. Code Documentation

Appendix H. Input Requirements

As an example of how the appendices may be used, consider the first flow in the Water Flow Model. In parentheses there will be a reference made to the appendix where the flow is located and the number in the appendix, i.e., (App. A, No. 1). The Flow F(P,1) can then be found in Appendix $A$, number 1 . For a secondary intermediate variable the same strategy will be used except that these variables will be found in Appendix B. In Appendices A and B the equation involving a flow or secondary intermediate variable will be stated, and the variables used will be defined for user convenience. Mnemonic names will not be used.

Appendix $\mathrm{C}$ contains variables discussed in this document and their mnemonic equivalents in the code are given as well as statement numbers where the mnemonic equivalent is defined.

Appendix D, Variable Library, contains only mnemonic names used in the actual code of the model. They have been defined for user convenience. 
Appendix $\overline{\mathrm{E}}$ contains the code of the TRU Biological Transport Model to be used with Appendices $\mathrm{C}, \mathrm{D}, \mathrm{F}, \mathrm{G}$, and $\mathrm{H}$ as well as having the actual prograin to reference.

Appendix $\mathrm{F}$ contains Plant Number Positions. In the program plants are simulated by punching a particular code in a position of an array that has 22 members. Positions 1 through 21 are listed with the particular plant that is being simulated.

Appendix $G$ is the Code Documentation. This contains numbers of statements in order and what is being done within those statements.

Appendix $\mathrm{H}$ is the Input Requirements for running the program. Input cards are listed in the proper order, and input that is required or that is optional is indicated. Mnemonic names are used.

\section{WATER FLOW MODEL}

The flow of water in the model is illustrated in Fig. 6 . The fiows $F(P, 1)$ and $F(P, 2)$ are dependent on several variable types:

$F(P, 1)=F(P, 2)=f\left(D_{1}, I_{1}, S_{1}, I_{6}, S_{2}, S_{3}, I_{4}\right)$

(App. A, No. 1)

These flows represent transport of water from precipitation events to soil and snow compartments during a given calendrical day $\left(I_{5}\right)$ :

$$
\begin{array}{rlrl}
F(P, 1) & =D_{1} I_{1} S_{1}\left(1+I_{8} S_{3}\right) & & I_{6}=\left|S_{2}\right| \\
& =0 & I_{8} \neq\left|S_{2}\right|
\end{array}
$$

(App. A, No. 2)

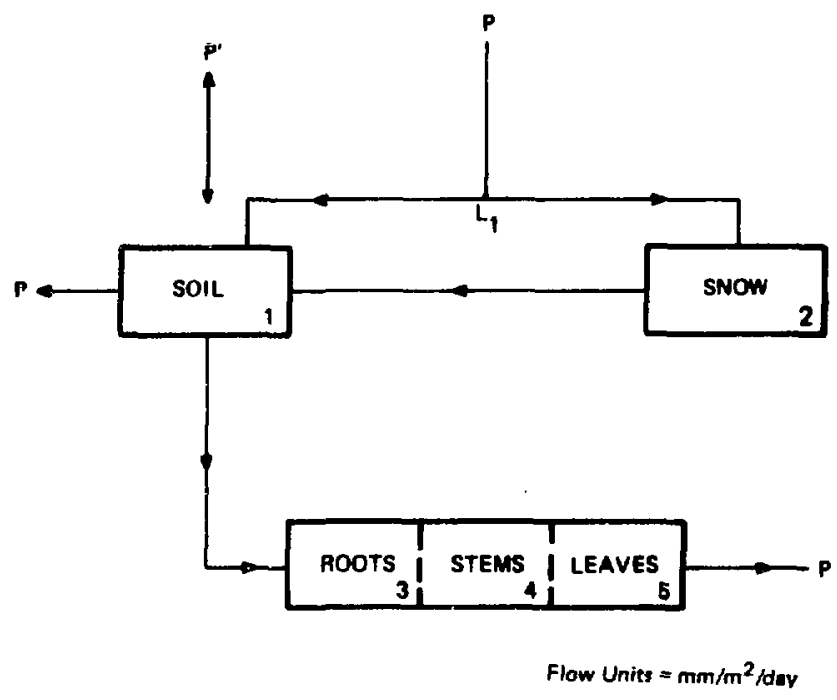

Fig. 6.

Water flow niodel. 
The decision $\left(L_{1}\right)$ which allocates precipitation as water or snow is based on daily temperature:

$$
\begin{aligned}
\mathrm{L}_{1} & =\mathrm{F}(\mathrm{P}, 1) & \mathrm{D}_{2}>0 \\
& =\mathrm{F}(\mathrm{P}, 2) & \mathrm{D}_{2}=0
\end{aligned}
$$

(App. B, No. 1) (Sec. III)

where the flows indicated are identical once $\left(L_{1}\right)$ has been executed.

The flow of precipitation to soil $\left(\mathrm{X}_{1}\right)$, and snow $\left(\mathrm{X}_{2}\right)$ compartments is based on the total number of precipitation events occurring within a given area that exceeds 0.1 inch $\left(\mathrm{I}_{4}\right),{ }^{12}$ a rainfall distribution fraction by month $\left(\mathrm{I}_{1}\right),{ }^{19}$ and an estimated coefficient of variation ${ }^{14}$ for the study area.

The second intermediate variable

$$
S_{1}=\frac{S_{1}}{\sum_{j=1}^{I_{4}} S_{j}}
$$

where each $S_{1}$ is a random number from an even distribution. The driving variable $D_{2}$ is based on a sinusoidal function: ${ }^{16.18}$

$\left.\mathrm{D}_{2}=\frac{\left(\mathrm{I}_{7}-\mathrm{I}_{8}\right)}{2} * \operatorname{Sin}\left(2 \pi^{*}\left(\mathrm{I}_{6}-\mathrm{I}_{8}\right) / 365\right)+1\right)+\mathrm{I}_{8}$

(App. B, No. 1) (Sec. II)

The temperature is corrected for elevation and latitudinal differences

$D_{2}=D_{2} I_{10}^{*}\left(I_{11}-I_{13}\right)$

(App. B, No. 2)

$\mathrm{D}_{2}$ is converted to centigrade and varied stoichastically on a monthly basis. ${ }^{3}$

$D_{2}=\left(D_{2}-32\right) * 5 / 9+S_{3} * I_{14}$

(App. B, No. 3) (Sec. II)

The decision to represent temperature trends on a monthly basis was based on observation of weather reports, although this analysis was not exhaustive; other trend lengths greater than one day could have been used but not as easily incorporated into the model code. The flow $F(2,1)$ is based on a snow melt function: ${ }^{17}$

$F(2,1)=C_{1} * X_{2}$

(App. A, No. 3)

The flow $F(1, P)$ is related to soil moisture status during any $F(P, 1)$ and $F(1,2)$

$$
\begin{aligned}
F(1, P) & =X_{1}+P-I_{15} & X_{1}+P>I_{15} \\
& =0 & X_{1}+P \leq I_{16}
\end{aligned}
$$

(App. A, No. 4) 
that is the moisture above the holding capacity in the top $250 \mathrm{~mm}$ of soil is lost to a runoff sink and not utilized in the model; adjustment for soli depth is considered in the next flow.

The flow $F(1,3 \cdot 4 \cdot 5)$ is related to soil depth, soil moisture, and ambient air temperature

$$
\begin{aligned}
F(1.3 \cdot 4 \cdot 5) & =I_{18} & & X_{1} \geq I_{18} \\
& =I_{18} * S_{1} / I_{18} & & I_{17} \leq X_{1} \leq I_{18} \\
& =0 & & X_{1} \leq I_{17}
\end{aligned}
$$

(App. A, No. 5)

where

$\mathrm{I}_{18}=\mathrm{C}_{2}+\mathrm{C}_{3} * \mathrm{D}_{2} \cdot{ }^{15}$

(App. B, No. 2)

The flow $F\left(P^{\prime}, 1\right)$ is an "apparent" flow of moisture from lower soil depths (greater than $250 \mathrm{~mm}$ ) to the upper $250 \mathrm{~mm}$ depth:

$F\left(P^{\prime}, 1\right)=\left(1-I_{3}\right)^{*} I_{20}$,

(App. A, No. 6)

v:here

$I_{3}=\left(\cdot / 1-\operatorname{EXP}\left(-C_{5} * I_{18}\right)\right)$.

(App. B, No. 1)

The latter factor was estimated from evapotranspiration studies of conifers in the Cascade Range, ${ }^{10}$ and is based on root distribution pattern and $\mathrm{O}_{2}$ utilization strategies by these plants. The relationship has been extended to include all plants in the model. Finally, the flow $F(5, P)$ or evapotranspiration is numerically identical to root absorption: $F(1,3 \cdot 4 \cdot 5)=F(5, P)$. As indicated by the model, only those flows pertinent to biomass flow or nutrient uptake were considered in the water flow model.

\section{BIOMASS FLOW}

The flow of biomass in the model is central to all other model flows. Also, biomass attributes which may or may not affect biomass flow are also interphased with this model such as management, ruminant herd; i.e., biomass flow generally parallels TRU flow which occurs concomitantly with it. A diagram of biomass flow is presented in Fig. 7. Internal flows of biomass occur during program operation, whereas peripheral biomass flow requires user option. Attribute interphasing may affect flow for various competing plant types, but not for a single type itself. The different strategies for plant growth requires that internal flow adapt simultaneously to any group of plants under simulation. This will become apparent as flow characteristics are described and both intermediate variables (I), and different constants (C) are employed in specific situations.

The flow $F(P, 5)$ or potential gross photosynthesis is represented by

$$
\begin{aligned}
F(P, 5) & =X_{6} * I_{21} * I_{22} * I_{23} & L_{2}=1 \\
& =0 & L_{2}=0
\end{aligned}
$$

(App. A, No. 7) 


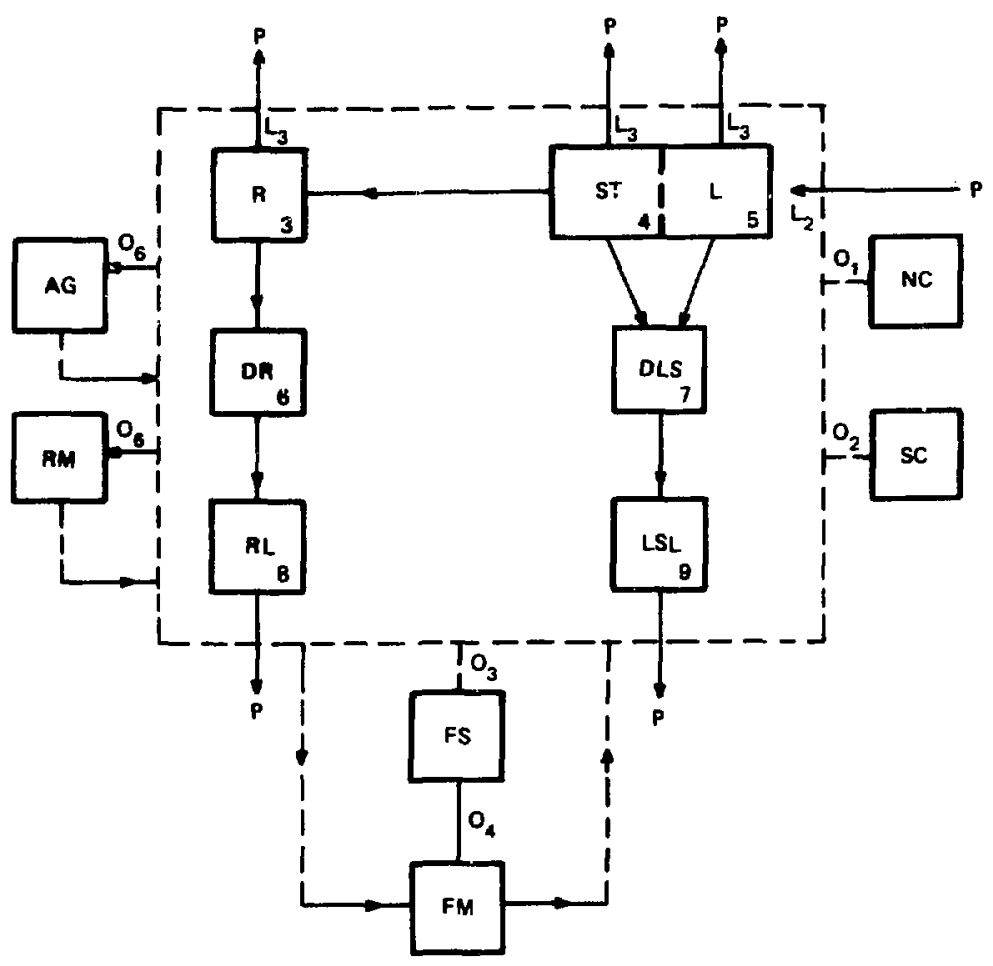

LEGEND

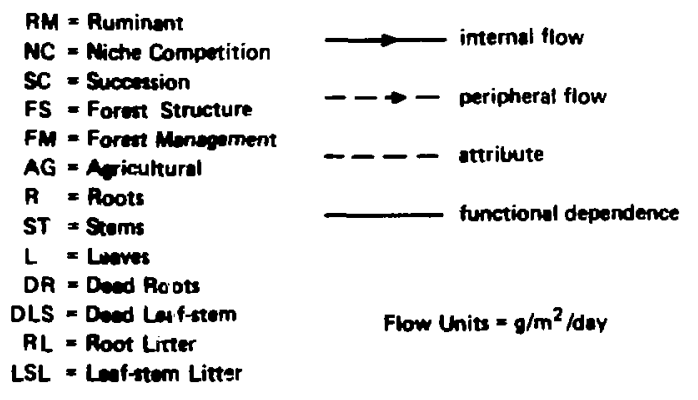

Fig. 7.

Biomass flow and peripheral attributes.

where

$$
\begin{array}{rlrl}
\mathrm{I}_{21} & =1-\left(\mathrm{D}_{2}-\mathrm{C}_{6}\right)^{2} / \mathrm{C}_{7} & & \mathrm{D}_{2}>\mathrm{C}_{8} \\
& =1-\left(\mathrm{D}_{2}-\mathrm{C}_{6}\right)^{2} / \mathrm{C}_{8} & \mathrm{D}_{2} \leq \mathrm{C}_{8}
\end{array}
$$

(App. B, No. 3) (Sec. II)

represents estimates of temperature effect on photosynthesis for different plant types within the model. ${ }^{16,19}$ 
The effect of soil moisture on photosynthesis is determined as

$$
\left.I_{22}=1-\operatorname{EXP}\left(-\left(C_{8} / I_{16}-I_{17}\right)\right) *\left(X_{1}-I_{17}\right)\right)+0.01 \quad \text { (App. B, No. 4) }
$$

where $\mathrm{X}_{1}$ refers in this case tu available soil moisture of the water flow model. This relation ${ }^{16}$ was adapted to plants other than perennial grasses by assessing their soil water dependence through tuning of the model, although more exhaustive investigations of this type are probably advisable for fine tuning of plants not directly comparable to those in the study area.

The effect of insolation on photosynthesis is described as follows:

$I_{23}=C_{10} * D_{3}$

(App. B, No. 5),

where the daily insolation

$D_{3}=\left(I_{24}-I_{26}\right) *\left(\left(\operatorname{Sin} *\left(2 *\left(I_{b}-I_{26}\right) / 365\right)\right)+I_{26} \quad\right.$ (App. B, No. 4) (Sec. II)

However, $D_{3} \leq 450.0$, or is set to that value if greater in calculating $\left(I_{23}\right)$ in agreement with IBP studies for light saturation of photosynthesis with Calvin type plants ${ }^{20}$ which have maximum efficiencies of about $0.45 \mathrm{~g} \mathrm{CH}_{2} \mathrm{O} / \mathrm{g}$ leaf biomass in temperature zone. ${ }^{13}$ Plant respiration is represented by:

$$
\begin{aligned}
\mathrm{F}(3, \mathrm{P})+\mathrm{F}(4, \mathrm{P})+\mathrm{F}(5, \mathrm{P}) & =\left(\mathrm{X}_{6}+\mathrm{C}_{11}{ }^{*} \mathrm{X}_{\mathrm{b}}\right) * \mathrm{C}_{12} * \operatorname{EXP}\left(\mathrm{C}_{18}{ }^{*} \mathrm{D}_{2}\right) * \mathrm{C}_{14} & \mathbf{L}_{\mathbf{3}}=1 \\
& =0 & \mathbf{L}_{\mathbf{3}}=0
\end{aligned}
$$

(App. A, No. 8)

The gate $\left(\mathrm{L}_{3}\right)$ is used in conjunction with agricultural crops to simulate planting and harvesting periods. Also, if $\left(C_{11}=0.0\right)$ as it is for perennial grasses and other forage crops, then root respiration $F(3, P)$ is uncoupled from above ground biomass:

$\mathrm{F}(3, \mathrm{P})=0.3^{*} \mathrm{X}_{3} * \mathrm{C}_{12} * \operatorname{EXP}\left(\mathrm{C}_{1 \mathrm{~s}} * \mathrm{D}_{2}\right) * \mathrm{C}_{14}$.

(App. A, No. 9)

For agricultural crops $\left(C_{11}=0.0\right)$ root and stem respiration biomass components are all grouped under $\left(X_{b}\right)$ or the leaf biomass itself. The maintenance biomass $\left(X_{5}+C_{11}{ }^{*} X_{8}\right)$ is observed to be equal to some multiple of leaf biomass $\left(\mathrm{X}_{\mathrm{b}}\right)$. The decision to adopt this strategy for woody plants, and all plants in general was based on experimentation with other strategies relating sapwood thickness among conifers ${ }^{4,21}$ and other structural studies. ${ }^{2}$ The most easily tuned strategy was adopted for simulating growth of Ponderosa pine $\left(C_{11}=3.0\right)$ and Pinon pine $\left(C_{11}=5.0\right)$ which indicated that maintenance biomass relative to leaf biomass increased with increasing adaptation to xeric environments as expected. Limited simulation experiments with Douglas-Fir $\left(C_{11}=2.0\right)$, and deciduous trees $\left(C_{11}=1.5\right)$ further support this strategy. The model thus functions by limiting growth rate and overall equilibrium biomass in tree species which have more water storage and absorbing potential, and therefore greater adaptability to xeric environments. More experimentation with the model in this regard is necessary.

The determination of leaf biomass in the model is not based on flow processes, but on a relationship depending on total above ground biomass for nonwoody plants:

$X_{b}=0.928 * I_{27}^{*} \operatorname{EXP}\left(-3.11 * I_{27}\right)+0.072 * I_{27}$ (App. B, No. 2) 
where

$I_{27}=\left(X_{4}+X_{8}\right) \quad($ App. B, No. 6);

that is leaf biomass is calculated on a daily basis. The ratio of total leaf biomass to total above ground biomass $\left(\mathrm{X}_{6} / I_{27}\right)$ with increasing biomass density produces a reversed sigmoid type curve which becomes constant $(0.072)$ at about $2000 \mathrm{~g} / \mathrm{m} .{ }^{2}$ It is intended to simulate the transformation of an herbaceous type plant into a woody or pithy structure. Quantitative information of this type was not found in the literature although numerous information was available on related root/shoot biomass ratios. However, proper uperation of the model for all types of plants does require a strategy of this type. Investigation of $\left(\mathrm{X}_{8} / I_{27}\right)$ ratios in selected plant types would be of great value in further verifying this strategy.

For woody perennial plants leaf biomass is limited by bud formation during the previous year

$$
\begin{aligned}
X_{5} & =C_{15} * I_{28} \\
& =X_{5} \text { (previous calculation) }
\end{aligned}
$$$$
\mathrm{X}_{6} \geq \mathrm{C}_{15} * \mathrm{I}_{28}
$$

$$
\mathrm{X}_{6}<\mathrm{C}_{15} * \mathrm{I}_{28}
$$

(App. B, No. 3) (Sec. V)

where $I_{28}$ equals the running average annual productivity

$i_{2 B}=\sum_{i=i}^{I_{s 0}} I_{2 g I} / I_{30}$

(App. B, No. 7)

and the current year productivity $\left(I_{28}\right)$ is related to actual net daily photosynthesis to be described later in this report. Whereas needle biomass in conifers is related to running av erage annual productivity in the model, leaf biomass in deciduous trees appears to be related to (1/2) of this quantity as judged by simulation experiments. Support for the conifer strategy comes from measurement of needle biomass density in different aged stands of Douglas Fir, ${ }^{29}$ and an extensive study of a Ponderosa Pine forest; ${ }^{28}$ the deciduous tree strategy is supported by leaf biomass estimates in a deciduous forest. ${ }^{24,28}$ In both cases the needle and leaf densities as well as productivities tend to reach or oscillate around an equilibrium value. A further control on leaf biomass for deciduous trees and shrubs is leaf drop:

$$
\begin{array}{rlrl}
\mathrm{X}_{5} & =\mathrm{X}_{3} & \mathrm{~L}_{4}=1 & \\
= & \mathrm{X}_{5} & \mathrm{~L}_{4}=0 ; \mathrm{I}_{31}+1<\mathrm{D}_{2}<\mathrm{I}_{32}+1 & \text { (App. B, No. 4) (Sec. V) } \\
& =1.0 & \mathrm{~L}_{4}=0 ; 1_{31}+1>\mathrm{D}_{2}>\mathrm{I}_{32}+1
\end{array}
$$

where leaf break and drop is simulated as an abrupt event at the beginning and end of frost-free period rather than a more gradual transition. This strategy was adopted to coincide with an average frost-free period or integral average growth activity period.

Actual net photosynthesis is estimated from potential net photosynthesis

$$
I_{34}=F(P, 5)-F(3, P)-F(4, P)-F(5, P)
$$

(App. B, No. 10) 
after a correction for water utilization efficiency for different plants at different times during the year. The amount $(\mathrm{m} \ell)$ of water required for each gram of photosynthate prod lced is estimated on a daily basis as $I_{3 b}=\left(C_{10}-C_{17}\right) /\left(I_{21}^{*} I_{22}\right)$ (App. B, No. 9), where the denominator is not allowed to fall below 0.01 . Hence, the actual net photosyrithesis is

$$
\begin{aligned}
I_{35} & =I_{34} * I_{36} * I_{37} & I_{33} * I_{34} \leq I_{20} * 10^{3} * I_{30} * I_{37} \\
& =I_{34} * I_{36}{ }^{2 *} I_{37}{ }^{2 *} I_{20} * 10^{3} /\left(I_{33} * I_{34}\right) & I_{33} * I_{34}>I_{20} * 10^{3} * I_{36} * I_{37}
\end{aligned}
$$

(App. E, No. 11)

where

$$
\begin{aligned}
\mathrm{I}_{38} & =\mathrm{I}_{36} & \mathrm{O}_{2}=1.0 \\
& =1.0 & \mathrm{O}_{2}=0.0
\end{aligned}
$$

and

$$
\begin{aligned}
I_{37} & =I_{37} & & O_{1}=1.0 \\
& =1.0 & & O_{1}=0.0
\end{aligned}
$$

(App. B, No. 16)

The intermediate variables $\left(I_{\mathbf{3 6}}, I_{37}\right)$ will be discussed when peripheral biomass flows are considered. The limitation of net photosynthesis by available water, and the utilization efficiency of the plant itself was observed to follow phenological development in range plants: ${ }^{27}$ a sinusoidal function was adopted in a grassland simulation study based on this yearly event. ${ }^{15}$ However, for modeling purposes it was reasoned that since both ambient temperature and available soil moisture affect overall photosynthesis, then they also affect water utilization efficiency as well as phenological development. Substitution of the interaction term $\left(I_{21} * I_{22}\right)$ into the model as described together with the maximum utilization efficiency given for range grasses yielded results which were in good agreement with the sinusoidal function previously adopted ${ }^{15}$ Consequently, the relationship was extended to include other plant types as well. However, maximum utilization efficiencies for plants other than range grasses were found by tuning. Again the experiments yielded a trend which showed decreasing water utilization efficiencies as one proceeded from xeric to less xeric plants. Actual evapotranspiration studies on plants of these types are required for further verification. The new strategy eliminates phenological development relationships which follow soil-moisture temperature markers. Finally, current year productivity $\left(I_{29}\right)$ mentioned earlier is summed on a daily basis

$$
I_{2 \theta}=\sum_{i=1}^{365} I_{s 61}
$$

for each day of a calendrical year. The latter includes both positive and negative (respiratory) estimates of $\left(I_{s b}\right)$. The second condition is present during the early growth phase of woody plants.

The flow $\mathrm{F}(4,3)$ or transport of photosynthate to roots from above ground parts depends on the sign of net photosynthesis 


$$
\begin{aligned}
F(4,3) & =C_{18} * I_{36} & & I_{36} \geq 0 \\
& =I_{36} *\left(X_{3} /\left(X_{3}+I_{27}\right)\right) & & I_{35}<0
\end{aligned}
$$

Photosynthate allocation of roots of grasses and trees ${ }^{16,24}$ were assigned according to what was estimated as the most probable allocation for the mature plant. All other allocations were determined from tuning exp rriments using grass and tree type plants as guides, and balancing with mortality losses to be $c^{\prime}$ insidered. More information of this type is required for further verification.

The mortality flows $F(4,7)$ and $F(5,7)$ representing stem and leaf biomass mortality, respectively, follow different strategies depending on whether:

1) the plant type is evergreen or deciduous,

2) an annual or perennial grass,

3) a harvested crop other thar grasses.

The combined flow:

$$
\begin{array}{ll}
\mathrm{F}(4,7)+\mathrm{F}(5,7)= & \\
0.693 * \mathrm{X}_{8} / 730+\mathrm{I}_{27} * \mathrm{I}_{38} & \text { a. } \mathrm{O}_{8}=0, \mathrm{~L}_{5}=0, \mathrm{~L}_{4}=1 \\
\mathrm{I}_{98} * \mathrm{I}_{27} & \text { b. } \mathrm{O}_{8}, \mathrm{~L}_{6}=0, \mathrm{~L}_{4}=0 \\
\mathrm{I}_{88} * \mathrm{I}_{27}+\mathrm{X}_{6} & \text { c. } \mathrm{O}_{8}=0, \mathrm{~L}_{6}=0, \mathrm{~L}_{4}=0, \mathrm{D}_{2}=\mathrm{I}_{32} \\
0.005 * \mathrm{I}_{27} & \text { d. } \mathrm{O}_{8}=0, \mathrm{~L}_{5}=1, \mathrm{~L}_{4}=0, \mathrm{C}_{28} \leq \mathrm{D}_{2} \leq \mathrm{C}_{21} \\
\mathrm{I}_{27} *\left(\mathrm{C}_{20} *\left(\mathrm{D}_{2}-\mathrm{C}_{21}\right)+\mathrm{C}_{22} * 0.05\right. & \text { e. } \mathrm{O}_{8}=0, \mathrm{~L}_{5}=1, \mathrm{~L}_{4}=0, \mathrm{D}_{2}>\mathrm{C}_{31} \\
\mathrm{I}_{27} *\left(\mathrm{C}_{29}-\mathrm{C}_{24} * \mathrm{D}_{2}\right) * 0.05 & \text { f. } \mathrm{O}_{6}=0, \mathrm{~L}_{5}=1, \mathrm{~L}_{4}=0, \mathrm{D}_{2}<\mathrm{C}_{28} \\
0.1 * \mathrm{I}_{27} & \text { g. } \mathrm{O}_{6}=1, \mathrm{~L}_{6}=1, \mathrm{~L}_{4}=0, \mathrm{I}_{5}=\mathrm{I}_{38}
\end{array}
$$

(App. A, No. 14)

The variable $\left(I_{38}\right)$ is estimated as:

$I_{s 8}=2 /\left(365 * C_{27}\right)$

(App. B, No. 18)

or the reciprocal of the mean average life span of the plant in question. The variable $\left(I_{38}\right.$ is applicable only to harvested crops with the exception of pasture grasses and alfalfa. The remaining relationships account for mortality losses at extremes of temperature,$^{18}$ or operate on a continuous basis as with conifers and evergreen shrubs. The conifer needles are estimated to have a half-life of two years, ${ }^{26}$ while shrub evergreen leaves are assigned one year half-lives. The total leaf biomass of deciduous trees is added to litter yearly at the end of the frost-free period $\left(\mathrm{I}_{32}\right)$; one-tenth of above ground biomass $\left(\mathrm{I}_{\mathbf{n}}\right)$ is converted to leaf plant material at harvest $\left(\mathrm{I}_{\mathrm{ga}}\right)$ for specific crops. 
The flow $F(3.6)$ is patterned after mortality losses of above ground biomass with some exceptions:

$$
\begin{array}{rlr}
F(3,6) & =\{F(4,7)+F(5,7)\} *\left[X_{3} / I_{27}\right] & \text { a. } \\
& \left.=\{F(4,7)+F(5,7)\}^{*}\left[X_{3} / I_{27}\right\rfloor+\right]_{38}^{*} X_{3} & \mathrm{~b} \\
& =X_{3} * I_{38} \\
& =X_{3}
\end{array}
$$

where conditions:

a. Plants $(1,2,4,5,6,9,10,12,14,15,16,17,20,21)$, see App. F.

b. Plants $(7,19)$

c. Plants $(3,8,11,13,18)$

d. Plants $(1,2,12), I_{5}=I_{30}$

With the exception of annual and perennial grasses and several harvested crops, most of the plants have been modeled with the same root mortality strategy: proportional to above ground mortality losses; the entire root biomass is converved to dead roots at harvest. The differences between annual and perennial plants were determined by simulation experiments, although the latter was based on mortality losses of above ground parts. ${ }^{15}$

The flows $F(7,9)$ and $F(6,8)$ were modeled to be boih temperature and soil moisture dependent:

$F(7,9)=I_{40} * I_{41} * X_{7}$

(App. A, No. 16), and

$\mathrm{F}(6,8)=\mathrm{I}_{\mathbf{4 0}} * \mathrm{I}_{\mathbf{4 1}} * \mathrm{X}_{8}$

(App. A, No. 17), where

$\mathrm{I}_{40}=0.004+0.0032 * \mathrm{D}_{2}$

(App. B, No. 19), and

$I_{11}=1.0+\left(1 /\left(I_{10}-I_{17}\right)\right) *\left(X_{1}-I_{17}\right)$

(App. B. No. 20)

Both types of flows were modeled after deromposition studies of range grasses ${ }^{15}$ and extended to all types of plants including conifers. Although they would be expected to over-estimate pine needle decomposition rates, simulation experiments did not bear this out sufficiently to modify the relationship for these plants. This could possibly be due to the rather infrequent occurrence of precipitation combined with rather cold winter months which could be masking different decomposition rates among plants. More information and testing is required in this regard.

The decomposition rate of both root and above ground humus are also modeled similarly:

$$
\begin{aligned}
& \mathrm{F}(8, \mathrm{P})=\mathrm{X}_{\mathrm{\theta}} * 0.3 * \mathrm{C}_{12} * \operatorname{EXP}\left(\mathrm{C}_{13} * \mathrm{D}_{2}\right) * \mathrm{C}_{14} \\
& \mathrm{~F}(9, \mathrm{P})=\mathrm{X}_{9} * 0.3 * \mathrm{C}_{12} * \operatorname{EXP}\left(\mathrm{C}_{13} * \mathrm{D}_{2}\right) * \mathrm{C}_{14}
\end{aligned}
$$$$
\text { (App. A, No. 18) }
$$ 
They are identical to the strategy used for determining root respiration rate in perennial grasses described earlier. Such a strategy assumes that humus decomposition or mineralization rate is equal to root cell respiration at the same temperature. Further experiments are required to fine tune this strategy with field observations of this type.

\section{TRU FLOW}

The flow TRU in this rnodel has been firmly coupled tc both water and biomass flows discussed earlier. So little is known of the behavior of TRU in ecosystems that it was assumed advantageous to vector it with flows which were more easily modeled and understood, provided that the coupling was reasonable and accurate. In addition to concomitant flow of TRU with water and biomass flow, other cycling behavior involving the surface of soil and plants, and the atmosphere itself was incorporated into the model (Fig. 8). However, the latter component was modeled in concentration units, and has other constraints to be described.

The flow of TRU into the system is largely dependent on user options of several types:

$$
\begin{array}{rlrl}
\mathrm{F}(\mathrm{P}, 12) & =\mathrm{I}_{43} & & \mathrm{~L}_{6}>0 \\
& =I_{44} & \mathrm{~L}_{7}>0 \\
& =\mathrm{I}_{45} & & \mathrm{O}_{4}=1, \mathrm{O}_{3}>1, \mathrm{O}_{4}=3
\end{array}
$$

where $I_{49}=T^{2} R U$ activity $/ \mathrm{m}^{3}$ from card input for year $\left(I_{40}\right)$ and day $\left(I_{6}\right)^{1}$ and $I_{44}=F u n c t i o n$ AIRAC $\left(C_{28}, I_{46}, I_{6}\right){ }^{1,2}$ The last option $\left(I_{45}\right)$ involves peripheral flow and will be discussed later in this report.

The flow $F(12, P)$ is considered to be instantaneous on a daily basis:

$F(12, P)=X_{12}=F(P, 12)$.

(App. A. No. 21)

Air concentrations are not preserved nor partly preserved from one iteration to the next, but are generated daily. This includes TRU contributions to $\mathrm{X}_{12}$ from all other sources which will now be considered. The flow of TRU from the soil surface (upper $2 \mathrm{~cm}$ ) is based on resuspension estimates for different fractional plant covers:

$$
\begin{array}{rlrl}
\mathbf{F}(10,12) & =\left(3 \times 10^{4 *}\left(\mathrm{I}_{\mathbf{4 2}} * \mathrm{X}_{10}\right)\right. & & \mathbf{I}_{\mathbf{4 8}}=0 \\
& =0 & \mathbf{L}_{\mathbf{8 8}}>0
\end{array}
$$

where $\left(I_{46}\right)$ indicates precipitation amounts, the constant $\left(3 \times 10^{4} \mathrm{~g}\right)$ is the mass of $1 \mathrm{~m}^{2} \mathrm{soil}, 2 \mathrm{~cm}$ deep, and

$I_{42}=2.5^{*} \operatorname{EXP}\left(-4.6^{*} I_{47}\right) \times 10^{-8}$

(App. B, No. 21)

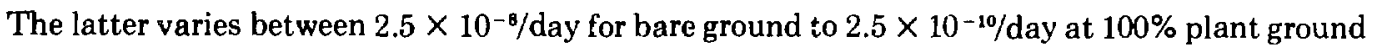
cover. The value at $16 \%$ ground cover corresponds to the resuspension rate estimated under desert conditions. ${ }^{29}$ The value at $100 \%$ ground cover compares with estimates in deciduous forests; ${ }^{30}$ resuspension is omitted during days when precipitation occurs. 


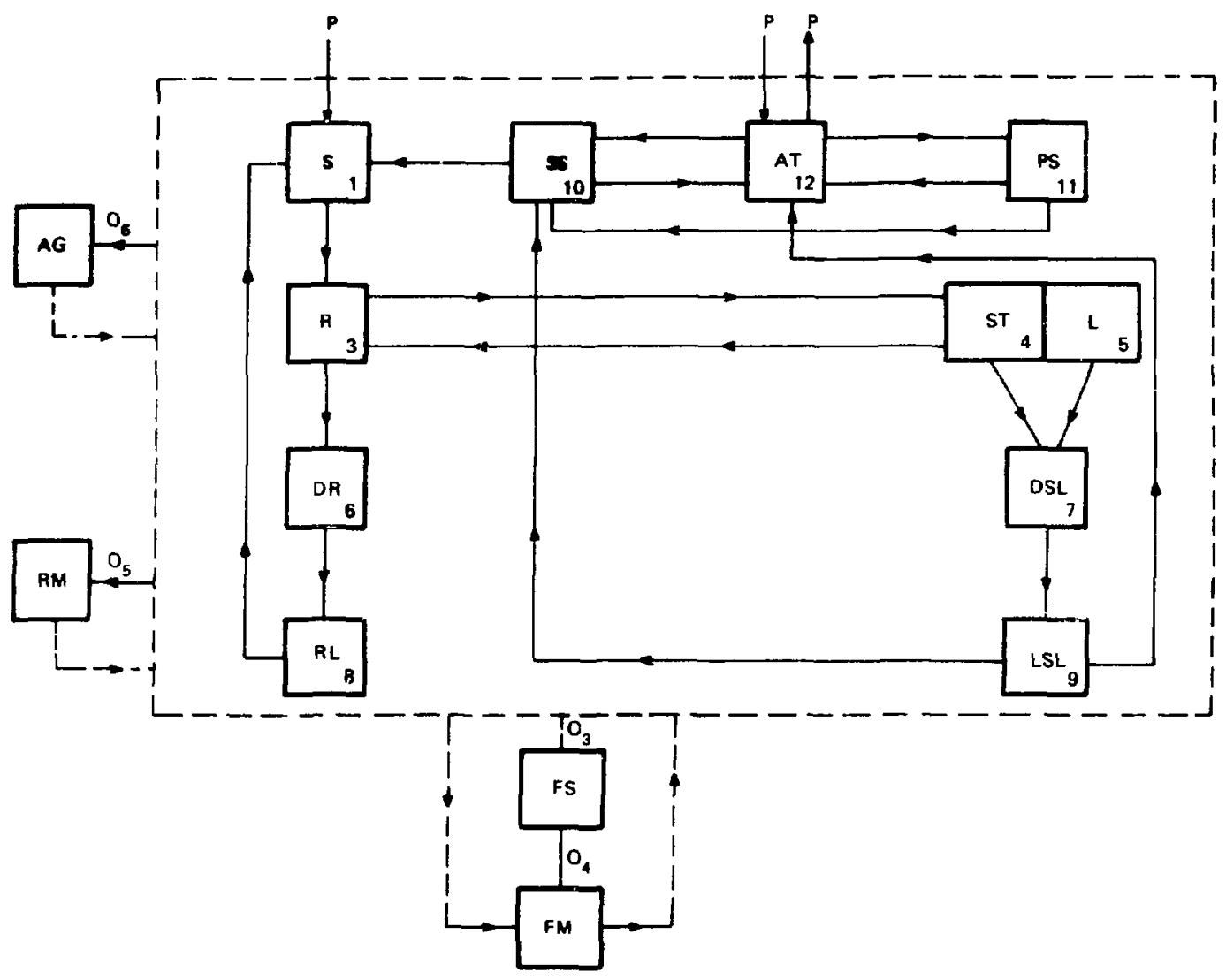

LEGEND

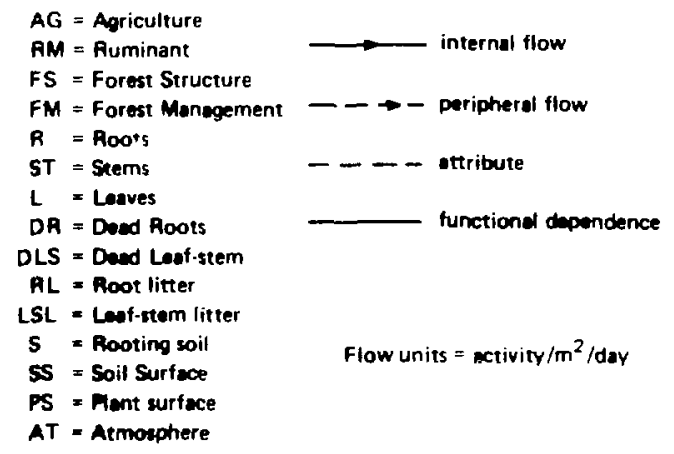

Fig. 8.

TRU flow and peripheral attributes. 
The estimate of plant cover $\left(\mathbf{I}_{47}\right)$ is obtained as follows:

$I_{17}=\left(1-\operatorname{EXP}\left(-1.54 * I_{26}\right) * I_{141}\right.$

(App. B, No. 22)

This estimate was made on the basis of tuning experiments with range grasses and conifers, and extended to other plants. More precise cover estimates for plant groupings, and for structural development of tree stands will be considered when peripheral flows are described.

The resuspension of TRU from plant surfaces is considered in an analogous fashion to soil resuspension:

$$
\begin{aligned}
\mathrm{F}(11,12) & =\mathrm{I}_{42} * \mathrm{X}_{11} & & \mathrm{I}_{48}=0 \\
& =0 & & \mathrm{I}_{48}>0
\end{aligned}
$$

The depositional flow $F(12,10)$ is based on a wind deposition velocity of $450 \mathrm{~m} /$ day - one-half the national average 'jecause of the low annual precipitation in the study area.

$$
\begin{aligned}
\mathrm{F}(12,10) & =0.0021^{*} \mathrm{X}_{12} / 3 \times 10^{4} & \mathrm{I}_{48}=0 & \\
& =0.7^{*} \mathrm{X}_{12}^{*}\left(1-\operatorname{EXP}\left(-0.6518^{*} \mathrm{I}_{48}\right)\right) / 3 \times 10^{4} & \mathrm{I}_{48}>0 & (\text { App. A, No. 24) }
\end{aligned}
$$

However, on days when precipitation occurs, up to $70 \%$ is deposited, ${ }^{31}$ depending on the amount of precipitation received $\left(\mathrm{I}_{48}\right)$. The ingrowth type factor in the relationship is based on an estimated optimum size rain $(8 \mathrm{~mm})$ which produces the maximum deposition noted, and where larger precipitation events do not increase deposition appreciably.

The deposition of particulates containing TRU on plant surfaces is related to the leaf area index for the given plant $\left(I_{48}\right)$ :

$$
\begin{aligned}
\mathrm{F}(12,11 ; & =0.0021 * \mathrm{X}_{12} * \mathrm{I}_{48} * 0.67 & \mathrm{I}_{48}=0 \\
& =0.7 * \mathrm{X}_{12} *\left(1-\operatorname{EXP}\left(-0.6518^{*} \mathrm{I}_{48}\right)\right) * \mathrm{I}_{48}, & \mathrm{I}_{48}>0
\end{aligned}
$$

The leaf index $I_{49}$ is estimated from a relationship established for conifer needles: ${ }^{3}$

$\mathbf{I}_{\mathbf{4}}=0.013 * \mathbf{I}_{\mathbf{B} 0}$

(App. B, No. 23)

where

$$
\begin{aligned}
& I_{50}=X_{6}+X_{7} \quad \text { a. Plants }(1,2,3,7,8,11,12,13,18,19) \text {, see App. } F
\end{aligned}
$$

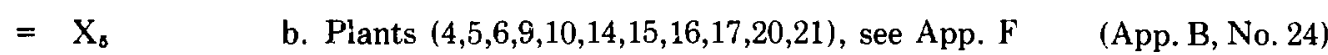

The plants considered under condition (a) are annual and perennial grasses, and crop plants where dead standing plant material may become appreciable; the deposition of TRU on leaf surfaces also assumes that about $67 \%$ of the particulates are retained under dry global fallout conditions. ${ }^{\text {ss }}$ 
The flow of TRU from plant to soil surfaces or washoff is partitioned into two strategies:

$$
\begin{aligned}
\mathrm{F}(11,10) & =(0.693 / 30) * \mathrm{X}_{11} / 3 \times 10^{4} & \mathrm{I}_{48}=0 \\
& =0.3 * \mathrm{X}_{11} *\left(1-\operatorname{EXP}\left(-0.6518^{*} \mathrm{I}_{48}\right) / 3 \times 10^{4}\right. & \mathrm{I}_{48}>0
\end{aligned}
$$

Dry fallout is estimated to have a 30-day half-time residence on plant surfaces, ${ }^{28}$ while during precipitation events, up to $30 \%$ of fallout may be removed.

Another source of resuspendable material is fine litter particles containing TRU in the flow:

$$
\begin{aligned}
\mathrm{F}(9,12) & =\mathrm{I}_{\mathbf{4 2}} * \mathrm{X}_{8} & \mathrm{I}_{48}=0 \\
& =0 & \mathrm{I}_{48}>0
\end{aligned}
$$

(App. A, No. 27)

The fine litter-humus compartment also transports TRU into the soil surface $\left(\mathrm{X}_{10}\right)$;

$\mathrm{F}(9,10)=0.3 * \mathrm{X}_{9} * \mathrm{C}_{12} * \operatorname{EXP}\left(\mathrm{C}_{19} * \mathrm{D}_{2}\right) * \mathrm{C}_{1} / 3 \times 10^{4}$

(App. A, No. 28)

The flow of TRU from the resuspendable horizon $\left(X_{10}\right)$ to the next $250 \mathrm{~mm}$ soil horizon below is formulated as:

$$
\begin{aligned}
\mathrm{F}(10,1) & =(0.693 / 700) * \mathrm{X}_{10} *\left(3 \times 10^{4} / 3 \times 10^{5}\right) & \mathrm{L}_{68}=0 \\
& =0.01 *\left(1-\operatorname{EXP}\left(-0.6518^{*} \mathrm{I}_{48}\right)\right)^{*} \mathrm{X}_{10} *\left(3 \times 10^{4} / 3.75 \times 10^{6}\right) & \mathrm{I}_{48}>0
\end{aligned}
$$

(App. A, No. 29)

Under dry conditions the flow of TRU from the resuspendable zone is estimated to occur with a 700-day half-life, although in earlier versions the model used a 70-day half-life. ${ }^{34}$ The former value is assumed to compromise discrepancies in reported flow rates. However, the more rapid flow in the presence of precipitation (by as much as a factor of 10 ) does allow some adjustment based on rainfall events.

The flow $F(P, 1)$ is not modeled; however, the presence of buried waste in various soil horizons or the discharge of TRU bearing solutions into soils would make this an important source of contamination. As with atmospheric inputs, TRU inputs into different soil levels $F(P, 1)$ is provided by user input option. ${ }^{1}$ The soil compartment has been partitioned into 31 sub-compartments below the $20 \mathrm{~mm}$ soil surface compartment $\left(\mathrm{X}_{10}\right)$; they consist of $250 \mathrm{~mm}$ deep horizons. The flow of TRU from these horizons to other compartments in the model is illustrated in Fig. 9. The determination of soil depth $\left(I_{54}\right)$ has been specified. In addition. several other soil characteristics are supplied by the user: the TRU activity in the ith soil horizon $\left(\mathrm{I}_{61,1}\right)$ TRU uptake coefficient $\left(I_{52,1}\right)$ and the root fraction in the ith soil horizon $\left(I_{53.1}\right)$; they can also be given a homogeneous value throughout the soil horizons it desired. ${ }^{2}$

The flow of TRU to roots from soil is thus a summation of level contributions:

$$
\begin{aligned}
\mathrm{F}(1,3) & =\sum_{\mathrm{i}=1}^{\mathrm{I}_{64}} \mathrm{I}_{61,1} * \mathrm{I}_{62,1} * \mathrm{I}_{58,1} * \mathrm{X}_{5} * \mathrm{I}_{20} * 10^{3} & & \mathrm{i} \leq \mathrm{I}_{65} \\
& =0 & & \mathrm{i}>\mathrm{I}_{65}
\end{aligned}
$$




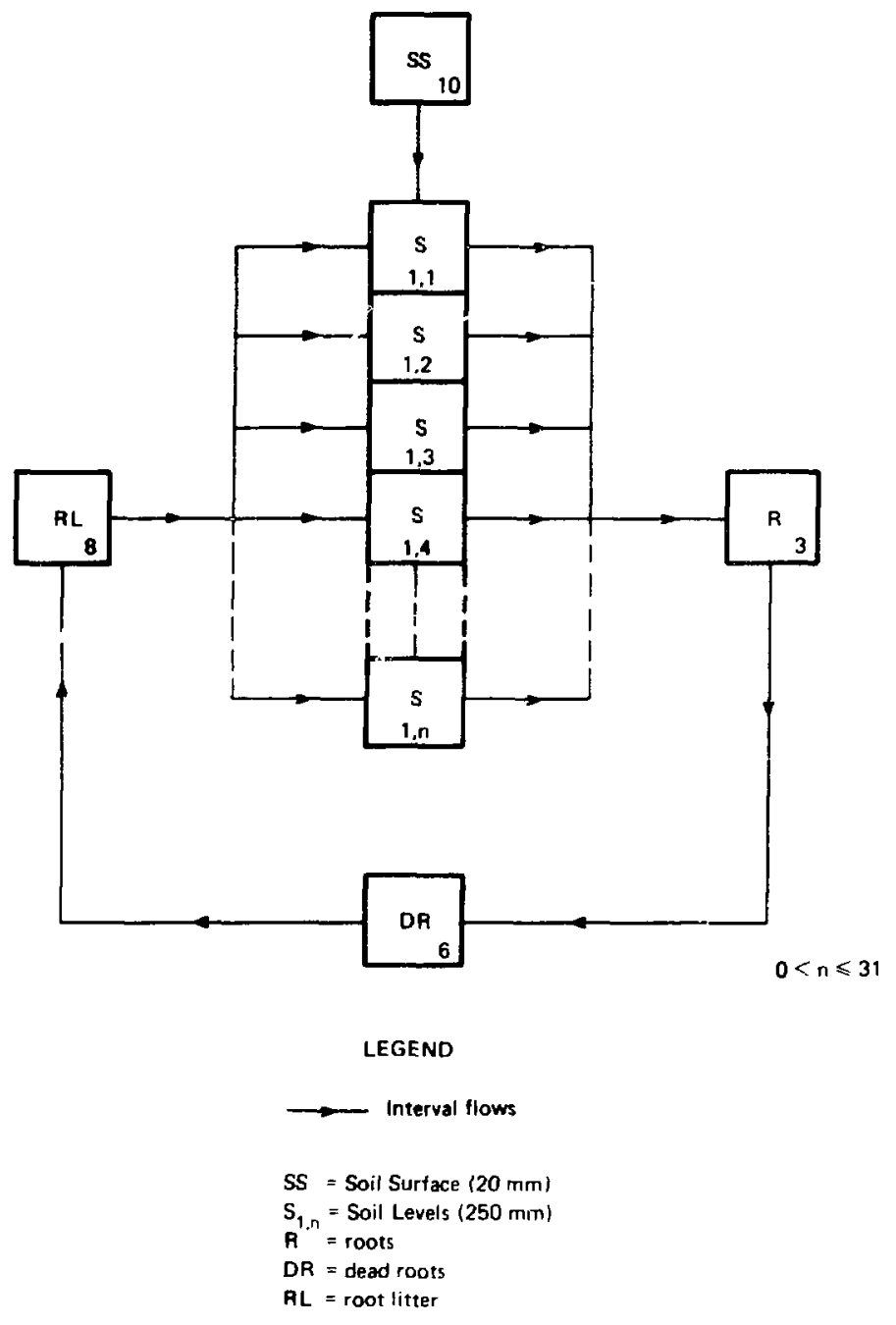

Fig. 9.

TRU flow through soil horizorts.

where the extent of root penetration $\left(I_{B S}\right)$ is calculated as:

$$
\begin{aligned}
\mathrm{I}_{65} & =\left(1-\operatorname{EXP}\left(-0.001 *\left(\mathrm{I}_{46}-1\right) * 365+\mathrm{I}_{5}\right)\right) * 9144 & \mathrm{C}_{27}>9.0 & \\
& =\left(1-\operatorname{EXP}\left(-0.001 * \mathrm{I}_{\delta^{\prime}}\right) * 9144\right. & \mathrm{C}_{27} \leq 9.0 & \text { (App. B, No. 25) }
\end{aligned}
$$

The rate of root penetration into the soil is an estimate based on exponential decrease of root biomass with depth in response to moisture and oxygen gradients. ${ }^{36}$ The relationship used for specifying root fraction distribution in soils at different depths is estimated by the following equation when not otherwise specified by the user:

$$
I_{57}=[\operatorname{EXP}(-0.004 * 250 *(i-1))-\operatorname{EXP}(-0.004 * 250 \mathrm{i})] / \operatorname{EXP}\left(-0.004 * I_{68}\right)
$$


where

$\left(2 \leq \mathrm{i} \leq \mathrm{I}_{\mathrm{si}}\right)$

(App. B, No. 27)

Investigation of this equation reveals that over $80 \%$ of tree roots are located in the upper meter of soil; $\left(I_{56}\right)$ limits the penetration of annual plants to the upper $2 / 3$ meter of soil for the most part.

The uptake of TRU by roots is controlled largely by actual evapotranspiration rate and current leaf biomass or leaf area index as described earlier; the uptake coefficient itself was estimased from tuning experiments involving clover cropping. ${ }^{36}$ Further testing of this hypothetical strategy would be helpful in verifying of TRU uptake from soil via water flux.

There are two outflows of TRU from the roots: the flow into above ground parts $F(3,4 \cdot 5)$, and the mortality flow $F(3,6)$. The latter is parallel to biomass flow:

$F\left(\cdots, \cdots=X_{3}^{*}(F 3,6) / \mathrm{X}_{6}\right)_{\mathrm{b}}$

(App. A, No. 31)

where the term $\left(\mathrm{F}(3,6) / \mathrm{X}_{6}\right)$ is a biomass flow parameter described earlier.

By the same analogy the decompositon of dead roots and flow of TRU to humus is:

$F(6,8)=X_{6} * I_{40} * I_{41}$

(App. A, No. 32)

The flow of TRU from root humus to soil, however, is partitioned to different soil levels in proportion to root fraction at any given level:

$\mathrm{F}\left(8, \mathrm{X}_{11}\right)=\mathrm{X}_{8} * \mathrm{I}_{52,1} * 3 * \mathrm{C}_{12} * \operatorname{EXP}\left(\mathrm{C}_{13} * \mathrm{D}_{2}\right) * \mathrm{C}_{14}, \quad 2 \leq \mathrm{i} \leq \mathrm{I}_{54}$

(App. A, No. 33)

as determined by $\left(I_{B b}\right)$. This relationship allows the accumulation of TRU in specific soil levels although it may not be present initially in given root horizons.

The exchange of TRU from below ground to above ground components has been modeled as a hypothetical transport system involving photosynthate production, maintenance biomass and root/shoot ratio:

$\mathrm{F}(3,4 \cdot 5)=\mathrm{X}_{3} * \mathrm{I}_{5 \mathrm{~B}} * \mathrm{I}_{27} / \mathrm{I}_{6 \mathrm{~B}}$

(App. A, No. 35)

where the exchange factor

$$
\begin{array}{rlrl}
I_{68} & =\left[\left\lfloor I_{60} /\left(X_{b}+C_{11} * X_{5}\right)\right] *\left[1-I_{60} /\left(X_{5}+C_{11} * X_{b}\right)\right]\right]_{b} & & I_{27}>1.0 \\
& =0 & I_{27} \leq 1.0
\end{array}
$$

and the subscript (b) indicates biomass flow parameters under consideration as described earlier. The translocate term is conditional:

$$
\begin{aligned}
\mathrm{I}_{60} & =|\mathrm{F}(4 \cdot 5,3)| & |\mathrm{F}(4 \cdot 5,3)| \leq\left(\mathrm{X}_{\mathrm{b}}+\mathrm{C}_{11} * \mathrm{X}_{\mathrm{b}}\right)_{\mathrm{b}}-1.0 \\
& =\left(\mathrm{X}_{6}+\mathrm{C}_{11} * \mathrm{X}_{6}\right)_{\mathrm{b}} & |\mathrm{F}(4 \cdot 5,3)|>\left(\mathrm{X}_{b}+\mathrm{C}_{11} * \mathrm{X}_{b}\right)_{\mathrm{b}}
\end{aligned}
$$


Thie flow of TRU is dependent on the root translocation under the assumption that both positive and negative values of net photosynthesis cause a flow of phloem into roots either in response to respiratory or storage conditions. The flow is conditioned by the relative storage capacity of roots with respect to above ground parts; the latter is made inversely proportional to root/shoot ratios as shown. Furthermore, it is assumed that in their counter-current flows Xylem, and phloem elements exchange TRU as they do many nuirients ${ }^{37}$ along their entire length; and therefore, flow is also somewhat dependent, although indirectly, to water flux or evapotranspiration process-etc., involved in plant growth rates. Another mechanism which is simulated by this strategy is the shunting of nutrients into roots during senescence or death of above ground biomass which occurs with perennial grasses. This process is mediated considerably in woody plants where appreciable storage occurs in the bole. Finally, the exchange is regulated during the early growth phases of plants when the root translocate/respiratory biomass ratio becomes significant or possibly greater than unity.

The flow of TRU from above ground parts as a result of mortality losses parallels biomass flow

$$
\left.F(4,7)+F(5,7)=\left(X_{4}+X_{5}\right) *((F 4,7)+F(5,7)) / I_{27}\right)_{b}
$$

where (b) indicates biomass flow processes.

Also, the flow of TRU from litter to humus is modeled parallel to biomass flow:

$F(7,9)=X_{7} * I_{40} * I_{41}$

(App. A, No. 37)

It is of importance to note that mortality but not respiratory processes cause a flow of TRU in contrast to biomass flow which occurs as a result of both. Hence, the increase in TRU concentrations in a plant tissue is due to respiratory as well as flow process. Also, the ratio of TRU/biomass resulting from both flows can be used to give estimates of TRU concentrations for any given compartment where TRU and biomass occur concomitantly.

\section{PERIPHERAL FLOWS OF WATER, BIOMASS, AND TRU}

The flow of TRU, biomass, and water through portions of the model which require user option to enter are termed peripheral flows. Within such processes one may increase the capabilities of flow prediction by formulating other attributes of plant biomass, or create other flows which are dependent on plant biomass as a driving variable. In addition, the capability of the model to simulate agricultural crops is dependent on this type of flow. Finally, peripheral flows interact with internal flows in a direct or indirect manner as will be described; the relationship between flows is illustrated in Figures 7 and 8 . The means by which a user may access peripheral flows have already been described, ${ }^{1}$ and some of the mechanics involved have been explained." This section of the report will consider the rationale used in the development of these modules.

\section{AGRICULTURAL PERIPHERAL FLOW}

Some interactions involving agricultural flow have already been mentioned: it interacts with water and biomass flow directly. For each crop plant simulated the module requires:

a) irrigation schedules $\left(I_{o 11}, i=1, j ; j \leq 16\right)$ 
b) harvesting schedules $\left(I_{a 21}, i=2, k ; k \leq 8\right)$

c) water application $\left(\mathrm{I}_{631}, \mathrm{i}=1, \mathrm{l} ; 1 \leq 16\right)$

where $\left(\mathrm{I}_{6 \mathrm{~B} 1}\right)$, and $\left(\mathrm{I}_{641}\right)$ represent planting and final harvesting dates for annual crops, respectively.

Irrigation schedules and concomitant water application interact with water flow by periodically augmenting soil moisture:

$$
\begin{aligned}
\mathrm{X}_{1} & =\mathrm{X}_{1}+25.4 * \mathrm{I}_{\theta 31} & \mathrm{I}_{5}=\mathrm{I}_{62 \mathrm{k}} \\
& =\mathrm{X}_{1} & \mathrm{I}_{5} \neq \mathrm{I}_{\mathrm{\theta 2k}}
\end{aligned}
$$

(App. B, No. 1) (Sec. V)

where $\mathrm{i}=\mathrm{ith}$ irrigation and water application. Planting dates if required and harvesting schedules interact specifically with biomass and TRU flow. The following biomass flow interactions are modeled ( $\mathrm{k}$ corresponds to the number of harvests in one year:
a) $F(4,7)+F(5,7)=0.1^{*} I_{27}$
b) $F(3,6)=X_{3}$
$k=2, I_{\sigma}=I_{62,1}$
c) $\mathrm{I}_{27}=1.0$
d) $X_{3}=1.0$
e) $F(P, 4 \cdot 5)=0$
$\mathrm{k}=2 ; \mathrm{I}_{\mathrm{BS1}}>\mathrm{I}_{6}>\mathrm{I}_{041}$
f) $F(4,7)+F(5,7)=0$
g) $\mathrm{I}_{27}=20.0$
$\mathrm{X}_{7}=\mathrm{X}_{7} *\left(20 / \mathrm{I}_{27}\right)$

$$
\mathrm{I}_{5}=\mathrm{I}_{\mathrm{B2k}} ; 2<\mathrm{k} \leq 8 ; \mathrm{I}_{27}>20.0
$$

(App. A, No. 38)

Analogous effects on $T R U$ flow are:
a) $\left(X_{4}+X_{6}\right)=0.0$
b) $\mathrm{X}_{3}=0.0$
$\mathrm{k}=2 ; \mathrm{I}_{\mathrm{s}}=\mathrm{I}_{64 \mathrm{k}}$
c) $\left(X_{4}+X_{5}\right)=\left(X_{4}+X_{5}\right) *\left(20 / I_{27}\right)$
d) $X_{3}=X_{3}^{*}\left(20 / I_{27}\right)$
$\mathrm{I}_{\mathrm{s}}=\mathrm{I}_{62, \mathrm{k}} ; 2 \leq \mathrm{k} \leq \mathrm{\sigma}, \mathrm{I}_{27}>20.0$

(App. B, No. 10)

The interaction of the agricultural module with all the types of flows used in the model are observed to be mostly mechanical in nature where materials balance is preserved as a result of human intervention in crop growth. 


\section{NICHE COMPETITION PERIPHERAL FLOW}

This module allows interaction of plants which are competing for the same resources with a similar strategy such as warm and cool season grasses; i.e., the interaction affects overall photosynthate production on a daily basis. Its effect on TRU flow is indirect, and has no effect on water flow as it now developed. Its effect on net photosynthesis for a given plant was estimated earlier as

$I_{96}=I_{35} * I_{36} * I_{37}$

(App. B, No. 12)

The competition factor involves a relative photosynthetic efficiency factor based on leaf biomass and other factors

$$
\begin{array}{rlrl}
\mathrm{I}_{861} & \left.\left.=\left[\left(\mathrm{I}_{88 \mathrm{~V}} /\left(\mathrm{C}_{271} * \mathrm{X}_{\mathrm{b}}\right)\right)\right] / \sum_{\mathrm{k}=1}^{\mathrm{n}} \mathrm{I}_{35 \mathrm{k}} / \mathrm{C}_{27 \mathrm{k}}: \mathrm{X}_{6 \mathrm{k}}\right)\right) & \mathrm{I}_{35}>0 & \text { (App. B, No. 15) } \\
& =1.0 & \mathrm{I}_{35} \leq 0
\end{array}
$$

where $\mathrm{n}=$ total number of plants in competition. ${ }^{1}$ The successional life-spans $\left(\mathrm{C}_{27}\right)$ have been found by tuning to be meaningful in simulating succession and will be discussed later in this report. The competition module has not jeen tested for plant groupings other than for warm and cool season grasses. ${ }^{\circ}$ Successional half-life terms have been equal for both types of plants. Validation experiments of this type are being planned to test this module with other plant groupings.

\section{SUCCESSION PERIPHERAL FLOW}

When accessed this module affects biomass flow directly on an annual basis through the production of a succession factor $\left(\mathrm{I}_{37}\right)$ which is used to adjust net daily photosynthesis:

$\mathrm{I}_{951}=\mathrm{I}_{351} * \mathrm{I}_{361} * \mathrm{I}_{971}$

The factor is related to total biomass, and successional life-span as a first approximation, and is the basis upon which the plants within a group are ranked according to successional advantage: the first ranking plants in the series have the maximum value of the annual running average ranking factor:

$I_{501}=\sum_{k=1}^{I_{46}}\left[\left(I_{271}+X_{91}+X_{6}+X_{7}+X_{8}+X_{8}\right) / C_{271}\right] / \sum_{k=1}^{I_{48}} k$

the first ranking plant has the second largest value of $\mathrm{I}_{50}$-etc., for $(\mathrm{K})$ plant groups. After obtaining a ranking $1,2,3-k$, a minimum cover factor is obtained for each group as follows, depending on rank: 


$$
\begin{aligned}
& I_{671}=I_{471} \\
& I_{671}=I_{471} *\left(1-I_{671}\right) \\
& I_{671}=I_{471} *\left(1-67 i-I_{673}\right) \\
& \cdot \\
& I_{87 \mathrm{~m}}=I_{47 \mathrm{~m}} *\left(1-I_{871}-I_{871}-\ldots I_{87 \mathrm{~m}}\right)
\end{aligned}
$$

m

(App. B, No. 31)

A potential succession factor is obtained for each plant by taking the product:

$I_{681}=\left(I_{671} / I_{471}\right) *\left(I_{681} / I_{69}\right)$

(App. B, No. 32)

The actual succession factor is estimated as:

$I_{37}=I_{68 i} \sum_{k=1}^{m} I_{68 k}$

(App. B, No. 17)

The strategy for estimating a succession factor is thus observed to involve:

1) a ranking of plants with a factor which is directly proportional to structural biomass, and inversely proportional to life-span

2) estimation of potential cover which could be occupied by all plants specified with those plants having the highest rank receiving first priority,

3) the calculation of a succession factor based on the ratio between the occupied cover and the potential cover which a plant would have without competition,

4) adjustment of the succession factor by a term which allows less successful types to occupy residual area not occupied by the dominants.

This strategy was developed and found satisfactory for simulating succession in ponderosa and pinon-juniper woodlands. It is currently being verified, and more validation is planned in the future for this hypothetical scheme.

\section{HERBIVORE PERIPHERAL FLOW}

This module affects both biomass and TRU flow strategies involved in the simulation of a ruminant herd. The module is divided into several different strategies for discussion purposes:

a) Food preference and feeding strategy

b) Herd and grazing characteristics 
c) Rumen function and animal growth

d) TRU uptake characteristics

The food preference scheme involves the classification of forage plants into groups depending on dietary choice:

$$
\begin{aligned}
\mathrm{I}_{711} & =0.7, \mathrm{i}=1 & \mathrm{I}_{70 \mathrm{j}, \mathrm{k}}=1 \\
& =0.2, \mathrm{j}=2 & \mathrm{I}_{70 \mathrm{j,k}}=2 \\
& =0.1, \mathrm{i}=3 & \mathrm{I}_{70 \mathrm{j}, \mathrm{k}}=3
\end{aligned}
$$

(App. B, No. 33)

where $(1 \leq \mathrm{i} \leq 3),(1 \leq \mathrm{j} \leq 21),(1 \leq \mathrm{k} \leq 4)$, and when $\mathrm{I}_{30 \jmath, \mathrm{k}}=4$, it refers to supplementary feed plants that will be considered later in this report. The preference fractions were estimated from reviews of food habits of wildlife. ${ }^{38,39}$ The total amount of forage available within each classification is estimated assuming a 3:1 preference of green to dry forage by the given animal: ${ }^{15}$

$I_{721}=\sum_{j=1}^{I_{731}}\left[\left(I_{74 j}\right) * X_{8 j}+\left(I-I_{74 j}\right) * X_{7 j} * X_{8 j} / I_{27 j}\right] * I_{78 j}$

(App. B, No. 36)

for all plants (j) included within the ith preference scheme, and where,

$I_{74 j}=3 * X_{5 j} /\left(3 * X_{8 j}+X_{7 j} * X_{8} / I_{271}\right)$

$I_{75 j}=\left(1-\operatorname{EXP}\left(-0.167\left(X_{8 j}+X_{7 j} * X_{8 j} / I_{27 j}\right)\right)\right)$

(App. B, No. 38

It should be noted that up to three preference classes are possible for native forage $\left(\mathrm{I}_{73}\right)$; however, it is possible that only one or two of these preferences are required. The preference fractions are then recalculated as:

$I_{711}=0.78$

$\mathrm{I}_{7 \mathbf{1}_{2}}=0.22 \quad \mathrm{I}_{7 \mathrm{~s}_{3}}=0$

(App. B, No. 34)

$\mathrm{I}_{\mathbf{7 1}_{1}}=1$

$$
\mathrm{I}_{73_{2}}=0.0, \mathrm{I}_{7 \mathrm{a}_{\mathrm{g}}}=0.0
$$

(App. B, No. 35)

Both $\left(I_{74}\right)$ and $\left(I_{78}\right)$ for different plants are estimated from grassland forage; ${ }^{16}$ the utilization factor does not allow foraging of plants to the point of extinction of biomass density for any given plant species considered in the simulation. Having estimated the total biomass density available for foraging in three preference classes $\left(I_{72}\right)$, other characteristics of the forage "mix" are estimated as weighted averages. These include:
a) nitrogen content of consumed forage
b) fraction of green biomass consumed
c) TRU specific activity in forage
d) TRU specific activity on forage surfaces
e) supplementary feed

These characteristics must be determined individually for all plants considered as forage prior to obtaining the weighted averages required. 
The nitrogen content for each plant is related to the growth rate of edible portions; ${ }^{18}$ in this model it is concidered the ratio of above ground translocate to the leaf biomass on a daily basis:

$$
\begin{aligned}
I_{781} & =C_{19} * I_{36} / X_{6} & & I_{701}>-0.05 \\
& =0.0 & & I_{701} \leq-0.05
\end{aligned}
$$

The nitrogen content of current photosynthate is estimated as:

$$
\begin{array}{rlrl}
\mathrm{I}_{771} & =3.0 & & \mathrm{I}_{781}>0.142 \\
& =(10.9) * \mathrm{I}_{781}+1.436 & \mathrm{I}_{701} \leq 0.142
\end{array}
$$

The nitrogen content of standing green biomass is estimated as

$I_{781}=I_{781} *\left(I_{79} / X_{61}\right)+\left(1-I_{79} / X_{51}\right)+I_{771}$

(App. B., No. 41)

where previous day leaf biomass is traisformed as

$$
\begin{aligned}
\mathrm{I}_{791} & =\mathrm{I}_{791} & \mathrm{I}_{791}<\mathrm{X}_{81} \\
& =\left(\mathrm{X}_{51}\right)^{2} / \mathrm{I}_{791} & \mathrm{X}_{701} \geq \mathrm{X}_{81}
\end{aligned}
$$

(App. B., No, 42)

for use in the relationship, and allows the proper symmetry of the equation to prevail. The nitrogen content of consumed forage is thus

$$
\begin{aligned}
\mathrm{I}_{881} & =\mathrm{I}_{741} * \mathrm{I}_{791}+\left(\mathrm{l}-\mathrm{I}_{741}\right) * \mathrm{C}_{31} & & \mathrm{I}_{781}>\mathrm{C}_{81} \\
& =\mathrm{C}_{31} & & \mathrm{I}_{781} \leq \mathrm{C}_{81}
\end{aligned}
$$

where the content is not allowed to fall below that of dry forage. The water content of the forage may also be estimated: ${ }^{15}$

$\mathrm{I}_{1621}=30^{*} \mathrm{I}_{\mathbf{8 1}}$

(App. B, No. 96)

The weighted fraction of green biomass in the ingesta of a given ruminant may be estimated if sufficient forage in each preference class is available. The amount of forage consumed from each preference group is estimated as

$I_{801}=I_{711} * I_{811}$

(App. B, No. 43)

where $I_{811}$ refers to intake by an animal of a given age category within the herd to be described later in this report.

The weighted nitrogen content of ingested forage over all preference groups for each age group of a given animal type is: 
$I_{82 k}=\sum_{i=1}^{n}\left(I_{801} / I_{81 k}\right) * \sum_{j=1}^{I_{n}}\left(I_{181}\right) / I_{72}$

(App. B, No. 44)

where,

$I_{151 j}=I_{86 j} *\left(X_{6 j} * I_{78 j}+\left(1-I_{78 j}\right) * X_{7 j} * X_{8 j} / I_{27 j}\right) * I_{78 J}$

(App. B, No. 95)

where the jth plant is in the ith food preference.

In similar fashion the fraction of ingesta as green biomass is estimated as

$\mathrm{I}_{88 \mathrm{k}}=\sum_{\mathrm{i}=1}^{\mathrm{n}}\left(\mathrm{I}_{80 \mathrm{j}} / \mathrm{I}_{\mathrm{BLk}}\right) * \sum_{\mathrm{j}=1}^{\mathrm{I}_{781}} \mathrm{I}_{\mathrm{Bdj}} ; \quad(1 \leq \mathrm{n} \leq 3)$

(App. B, No, 4h)

and

$I_{84 j}=I_{74 j} * X_{8 j} * I_{78} / I_{72}$

(App. B, No. 47)

for all plants ( $\mathrm{j}$ ) within the ith preference scheme.

Parallel types estimated for TRU specific activity of external and interna' origin are given as

$I_{85 k}=\sum_{i=1}^{n}\left(I_{90} / I_{81 k}\right) * \sum_{j=1}^{I_{73}} I_{80 j}$

(App. B, No. 48)

$I_{87 \mathrm{k}}=\sum_{\mathrm{i}=1}^{\mathrm{n}}\left(\mathrm{I}_{801} / \mathrm{I}_{81 \mathrm{k}}\right) * \sum_{\mathrm{j}=1}^{\mathrm{I}_{79}} \mathrm{I}_{88 \mathrm{~s}}$

(App. B, No. 50)

respectively, where

$I_{86 j}=I_{78 j} * I_{74 j} *\left(\left(X_{6} *\left(X_{6 j}+X_{6 j}\right)_{p}+\left(1-I_{74 j}\right) * X_{7} * X_{6} *\left(X_{7}\right)_{p} / I_{27}\right) I_{72} \quad(A p p . B\right.$, No. 49)

$I_{88 j}=I_{70 j} * I_{71 j} *\left(\left(X_{s} * X_{11 j}\right)_{p}+\left(1-I_{74 j}\right) * X_{7} * X_{b} *\left(X_{11}\right)_{p} / I_{27} / I_{721}\right.$

(App. B, No. 51)

and (p) subscript indicates TRU pool; others refer to biomass pool. The variables $\left(\mathrm{I}_{b 1}, \mathrm{I}_{B 2}, \mathrm{I}_{88}, \mathrm{I}_{B 8}\right.$, and $I_{87}$ ) are required for the simulation of rumen function to be described later in this report. ${ }^{16}$

Another source of forage included in the model is that of supplementary feed crops which are also simulated. Harvesting scheclules are used to gate accumulation of supplementary feed of various kinds throughout the year for a given animal type:

$I_{88: 77}=\sum_{i=1}^{I_{78}}\left(I_{27}-20+\left(\left(I_{27}-20\right) / I_{27}\right) * I_{801} * 4047\right.$

(App. B, No. 52) 
for all plants. In addition, the specific activity of TRU in supplementary feed is estimated as

$I_{01 \mathrm{~m}}=\sum_{\mathrm{i}=1}^{\mathrm{I}_{73}}\left(\left(\mathrm{X}_{61}+\mathrm{X}_{61}+\mathrm{X}_{71}\right)_{\mathrm{p}} /\left(\mathrm{I}_{271}+\mathrm{X}_{71}\right) *\left(\mathrm{I}_{211}-20\right) / \mathrm{I}_{271}\right) * \mathrm{X}_{71} * \mathrm{X}_{901} * 4047$

(App. B, No. 53)

and on supplementary feed as

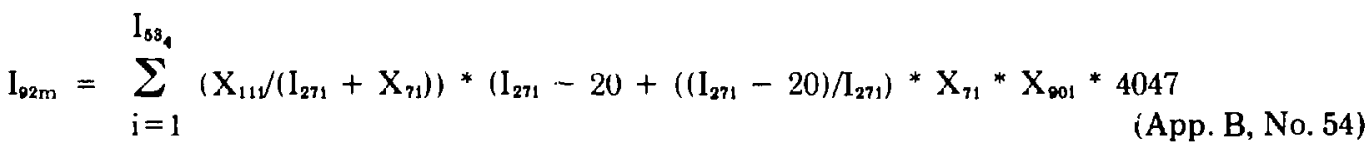

Previous year harvests and specific activities are preserved as $\left(I_{03}\right),\left(I_{04}\right)$, and $\left(I_{28}\right)$ for total biomass, TRU specific activity in plant, and TRU specific activity on the surface of the plant, respectively. These and current year values are used to estimate the TRU specific activities in and on piants on a daily basis for consumption:

$I_{90 m}=\left(I_{01 m} * I_{80 m}+I_{04 m} * I_{98 m}\right) /\left(I_{89 m}+I_{93 m}\right)$

(App. B, No. 55)

$I_{97 m}=\left(I_{92 m} * I_{80 m}+I_{8 b m} * I_{98 m}\right) /\left(I_{98 m}+I_{98 m}\right)$

(App. B, No. 56)

The amount of supplementary feed required for domestic animals on a daily basis will be discussed later in this report.

The relationship between forage related intermediate variables and flow of biomass and TRU into a simulated ruminant herd is shown in Fig. 10 for one animal from a specific age group. Whereas biomass type input $\left(\mathrm{I}_{\mathbf{B} 1}, \mathrm{I}_{\boldsymbol{8 0}}\right)$ is required for simulating biomass flow, other attributes of forage including nitrogen content and green biomass fraction also affect biomass flow into the herd. TRU flow largely parallels biomass; however, additional compartments are required to model TRU transport from soil and the atmosphere $\left(\mathrm{I}_{98}\right)$.

The simulated herd is characterized ${ }^{1.2}$ by

a) herd size

b) population structure

c) biomass structure

d) metabolic strategy

Herd size $\left(I_{1001}\right)$ is variable depending on yearly $\left(L_{a}\right)$ and daily $\left(L_{0}\right)$ gates supplied by the user. ${ }^{1,2}$ The population structure is based on an estimated livestock structure or wild ruminant structure $\left(C_{32}\right)$. Hence, the number of animal units of a given age group

$\mathrm{I}_{101 \mathrm{~J}}=\mathrm{C}_{32 \mathrm{~J}} * \mathrm{I}_{100 \mathrm{~J}}$

(App. B, No. 57)

where $\mathrm{j}$ refers to the $\mathrm{jth}$ age group within the herd. The biomass of an animal unit within each age group is estimated initially by the relationship, $\mathrm{C}_{s \mathrm{sj}}{ }^{*} \mathrm{I}_{101 \mathrm{j}}$, where $\mathrm{j}$ refers to the jth age category.

The metabolic strategy used in this model is to simulate rumen function ${ }^{16}$ in one animal unit of each age group and to apply the result to all other animal units within the age group. Hence, all animal units within a given age group are considered identical, and materials balances of TRU and biomass are based on multiples of a single estimate in each case. 

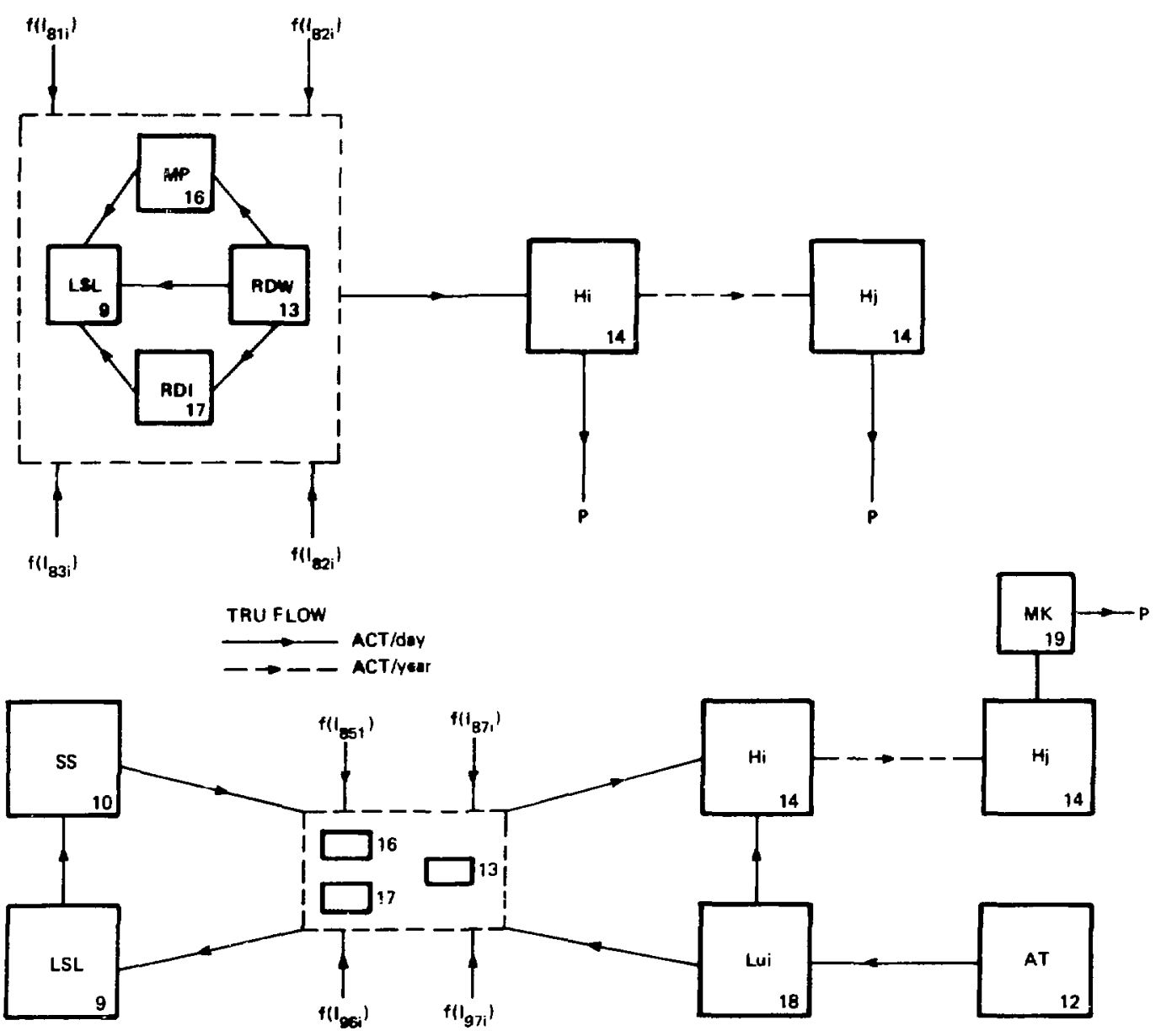

LEGEND

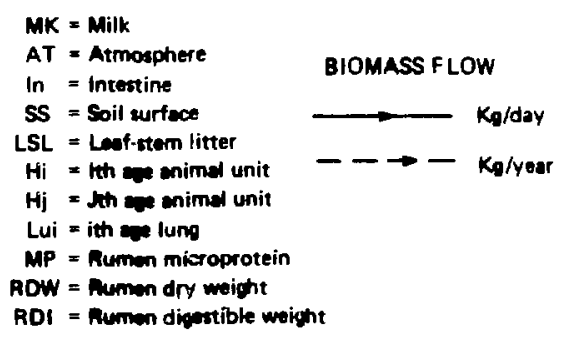

Fig. 10.

Biomass and TRU flow in a ruminant herd animal. 
Rumen function itself operates on a daily basis and is highly dependent on forage attributes described previously. The rumen may be described as compartment which has a certain capacity for forage that increases with animal unit biomass increase:

$\mathrm{I}_{1041}=0.16 * \mathrm{I}_{1031}$

where initially,

$I_{1031}=\left\lfloor C_{334} * I_{1021}\right]^{0.76}$,

and thereafter,

$I_{1031}=\left\lfloor X_{141}\right\rfloor^{0.75}$

(App. B, No. 58)

for the ith age group.

The amount of forage consumed by the animal unit on a given day is:

$F(P, 13)=I_{81}=\left(I_{104 k}-I_{110 k}\right)\left(1.0-0.3 * I_{82}\right)$

(App. A, No. 39)

$I_{1101}=X_{181}+X_{181}+0.001 * X_{101}$

(App. B, No. 63)

The digestible portion of intake is related to green-dry fractions in the forage:

$F(13,17)=I_{105}=\left(1-I_{831}\right) * 0.45+I_{831} * 0.7$

(App. A, No. 40)

The total amount of energy derived from this fraction is:

$\mathrm{I}_{108}=\mathrm{I}_{105} * \mathrm{I}_{81} * 4300$

(App. B, No. 60)

The microprotein derived fromthe intake is related to the nitrogen content $\left(\mathrm{I}_{82}\right)$, and the energy derived from the digestible fraction:

$\mathrm{F}(13,16)=\mathrm{I}_{107}=\mathrm{I}_{108} * \mathrm{I}_{\mathrm{i} 69}$

where

$$
\begin{aligned}
l_{15 s} & =0.456 & & I_{821} \geq 1.8 \\
& =0.023 *\left(I_{821}-0.6\right)+0.0186 & & 0.6 \leq I_{821}<1.8 \\
& =0.031 * I_{821} & & 0 \leq I_{821}<0.6
\end{aligned}
$$

and

$$
\begin{array}{rlrl}
\mathrm{I}_{821} & =\mathrm{I}_{821} & \mathrm{I}_{821}>\mathrm{C}_{31}, \mathrm{i}>1 \\
& =3.0 & \mathrm{i}=1 \\
& =\mathrm{C}_{31} & & \mathrm{I}_{821} \leq \mathrm{C}_{31}, \mathrm{i}>1
\end{array}
$$


The nitrogen level in forage is not allowed to drop below that in dry forage $\left(\mathrm{C}_{31}\right)$; also the nitrogen content of forage for the first age group is maintained at a level estimated from consumption of milk during weaning. The total amount of microprotein in the rumen initially is set at:

$\mathrm{X}_{1 \mathrm{es}}=200 * \mathrm{C}_{\mathbf{2 s} 1}$

The flow of microprotein from the rumen to the humus compartment is:

$F(16,9)=\left(0.52 * X_{18}\right) * 0.75$

(App. A, No. 42)

The fermentation digestion rate is related to microprotein/metabolic weight ratio:

$I_{1001}=\left(X_{101} / I_{1001}\right) * 0.04$

(App. B, No.61)

The rumen exit rate is thus:

$$
\begin{array}{rlrl}
I_{100} & =0.25+I_{108} & & I_{108} \leq 0.95 \\
& =0.95 & I_{: 08}>0.95
\end{array}
$$

(App. B, No. 62)

where, however, a certain fraction of the psssed food is fermented and is considered potentially digestible. The net flow of digestible material is thus incremented as:

$F(13,17)=F(13,17)+I_{1081} * I_{1081} * F(13,17)$

(App. A, No. 43)

The exit of the digestible forage portion from the rumen is then estimated as:

$\mathrm{F}(17,9)=\left(\mathrm{I}_{1001} * \mathrm{X}_{17}\right) * 0.75$

(App. A, No. 44)

The flow of undigested food from the rumen is estimated as:

$F(13,9)=\left(I_{1081} * X_{18}\right) * 0.75$

(App. A, No. 45)

The fraction of fermentation products as fatty acids is a function of the nitrogen content of the forage intake:

$$
\begin{aligned}
\mathrm{I}_{112} & =0.747-0.15^{*}\left(\mathrm{I}_{821}-0.6\right) & & 0.6 \leq \mathrm{I}_{821} \leq 1.8 \\
& =0.747 & & \mathrm{I}_{821}<0.6 \\
& =0.591 & & \mathrm{I}_{821}>1.8
\end{aligned}
$$

(App. B, No. 64)

The total energy derived from fatty acid metabolism is estimated as:

$I_{112}=I_{108} * I_{1081} * I_{111}$

(App. B, No.65)

The total energy derived from passed food is estimated as:

$$
I_{118}=[0.25 *(F(13,9)+F(17,9))] * 4300
$$


The total energy derived from microprotein is:

$I_{114}=0.65 * X_{10} * 4.3 * 0.52$

(App. B, No.67)

The total amount of energy available for metabolism is thus:

$I_{113}=I_{112}+I_{113}+I_{114}-125 * I_{1091}$

(App. B, No. 68)

The present strategy for supplementary feeding is made applicable while an animal is actively grazing in the simulation or is on supplementary feed. It may interact with gains or losses ty an animal unit and affect TRU flow. The amount of supplementary feed is averaged over the grazing periods, and is set equal to the basal respiratory term in mass equivalents on a daily basis for animals not on range:

$I_{1101}=125 * I_{1031}$

or

$I_{1101}=I_{118}$

(App. B, No. 69)

for animals on range when respiration exceeds caloric intake.

The weight gain by an animal unit depends on its biomass relative to the biomass of a mature weight animal unit. The additional maintenance factor is estimated as:

$$
\begin{array}{rlrl}
\mathrm{I}_{1171} & =\infty & \mathrm{I}_{120} \geq 1.0 \\
& =3.74+10.52^{*} \mathrm{I}_{120} & & 0.75 \leq \mathrm{I}_{120}<1.0 \\
& =3.74+5.26 * \mathrm{I}_{120} & & 0.5 \leq \mathrm{I}_{120}<0.75 \\
& =1.5 & & 0.0 \leq \mathrm{I}_{120}<0.5
\end{array}
$$

(App. B, No. 70)

where,

$\mathrm{I}_{118}=\mathrm{X}_{4} /\left(\mathrm{C}_{98_{8}} * \mathrm{I}_{1021}\right)$

(App. B, No. 71)

The actual weight gain by an animal unit on a daily basis is:

$$
\begin{aligned}
\mathrm{F}(13 \cdot 16 \cdot 17,14) & =0.0 & & \mathrm{I}_{1171}=\infty \\
& =\mathrm{I}_{1183} / 1700 & & \mathrm{I}_{1171} \leq 0.0 \\
& =\mathrm{I}_{1161} / \mathrm{I}_{1171} * 1700 & & 0 \leq \mathrm{I}_{1171}<1.0
\end{aligned}
$$

The total amount of forage consumed by an animal in a given age group may now be estimated as:

$$
I_{1181}=I_{s 8 k} * I_{s 1 k} * 1000+\left(1-I_{8 s k}\right) * 1000
$$


and the number of meters grazed is estimated as:

$I_{1191}=I_{1181} \sum_{j=1}^{3} I_{725}$

(App. B, No. 73)

and the total number of $\mathrm{m}^{2}$ grazed by all animals units of a given age class is:

$\mathrm{I}_{120\}}=\mathrm{I}_{1201} * \mathrm{C}_{821} * \mathrm{I}_{1011}$

(App. B, p. 13, No. 74)

The number of animal units/grazed area for an age group (i) is:

$I_{1211}=I_{1011} /\left(I_{1221} * C_{34}\right)$

(App. B, No.75)

The latter is used to estimate the amount of biomass removed from each plant type in the forage scheme:

$\mathrm{F}(4 \cdot 5,13 \cdot 16 \cdot 17)=\mathrm{I}_{83 \mathrm{k}} *\left(\mathrm{I}_{1231} / \mathrm{I}_{72 \mathrm{~m}}\right) *\left(\mathrm{I}_{80 \mathrm{~m}} / \mathrm{I}_{80 \mathrm{k}}\right) * \mathrm{I}_{121 \mathrm{k}}$

(App. A, No. 47)

where

$I_{1291}=\left(I_{741} * X_{81}+\left(1-I_{741}\right) * X_{71} * X_{81} / I_{271}\right) * I_{781}$

(App. B, No. 76)

Also:

$\mathrm{F}(7,13 \cdot 16 \cdot 17)=\left(1-\mathrm{I}_{88 \mathrm{~K}}\right) *\left(\mathrm{I}_{12 \mathrm{~g} /} / \mathrm{I}_{72 \mathrm{~m}}\right) *\left(\mathrm{I}_{80 \mathrm{~m}} / \mathrm{I}_{\mathrm{Bok}}\right) * \mathrm{I}_{121 \mathrm{k}}$

(App. A, No. 48)

TRU flow in the module is concomitant with biomass flow and is concerned primarily with the transport across the rumen wall. As described earlier TRU uptake is divided into supplementary and native forage pathways. The total amount of TRU ingested from native forage is estimated as:

$I_{134 k}=\left(I_{100 k} / I_{120 k}\right) *\left(I_{120 k}\right) *\left(I_{83 k}+I_{87 k}\right)+\left(\left(I_{128 k}-I_{1001}\right) / I_{128 k}\right) *\left(I_{110 k}\right) *\left(I_{91 k}+I_{02 k}\right) * I_{120}$

(App. B, No. 77)

If respiration exceeds caloric intake, then the TRU uptake is incremented by:

$I_{1241}=I_{1241}+\left|I_{1101} / 4.3\right| * C_{821}^{*}\left(I_{91 \mathrm{~K}}+I_{82 k}\right) * I_{128}$

(App. B, No. 78)

The total amount of supplementary feed consumed by a given age group is:

$I_{128 k}=I_{110 k} *\left(\left(I_{198 k}-I_{1001} / I_{125 k}\right) * C_{821}\right.$

(App. B, No. 79)

If respiration exceeds caloric intake, then consumed feed is incremented as:

$I_{1261}=I_{1281}+I_{1005} *\left|I_{1181} / 4.3\right|^{*} C_{82 k}$

(App. B, No. 80) 
The TRU flow from green biomass into the rumen is:

$\mathrm{F}(4 \cdot 5,13 \cdot 16-1 \mathrm{i})\left(\mathrm{X}_{4}+\mathrm{X}_{6}\right) *(\mathrm{~F}(4 \cdot 5,13 \cdot 16 \cdot 17))_{6} / \mathrm{I}_{27}$

(App. A, No. 49)

where the (b) subscript indicated biomass flow. Similarly, TRU flow from dry biomass is:

$\mathrm{F}(7,13 \cdot 16 \cdot 17)=\left(\mathrm{X}_{7}\right) *\left(\mathrm{~F}(7,13 \cdot 16 \cdot 17) / \mathrm{X}_{7}\right)_{\mathrm{b}}$

(App. A, No. 50)

and

$F(11,13 \cdot 16 \cdot 17)=X_{11} *\left[\left(F(7,13 \cdot 16 \cdot 17)+F(4 \cdot 5,13 \cdot 16 \cdot 17) / I_{27}+X_{7}\right)\right]_{b}$

(App. A, No. 51)

which is the transport from plant surfaces into the rumen. In this module the flow of TRU into the rumen from soil depends on the type of plant being consumed. For a given plant the amount, ${ }^{40}$ of soil ingested while foraging is estimated:

$$
\begin{array}{rlrl}
\mathrm{I}_{128 \mathrm{k}} & =0 & \mathrm{~L}_{5} & =0 \\
& =\left(\mathrm{I}_{800} / \mathrm{I}_{78 \mathrm{k}}\right) * \mathrm{X}_{14 \mathrm{k}} * 3 & \mathrm{~L}_{5}=1, \mathrm{I}_{27}+\mathrm{X}_{7}<20 \\
& =\left(\mathrm{I}_{801} / \mathrm{I}_{78 \mathrm{k}}\right) * \mathrm{X}_{14 \mathrm{k}} * 20 /\left(\mathrm{I}_{27}+\mathrm{X}_{7}\right) & \mathrm{L}_{5}=1, \mathrm{I}_{27}+\mathrm{X}_{2} \geq 20
\end{array}
$$

(App. B, No. 81)

The flow of TRU into the rumen is:

$\mathrm{F}(10,13 \cdot 16 \cdot 17)=\mathrm{I}_{128 \mathrm{k}} * \mathrm{C}_{32 \mathrm{k}} * \mathrm{I}_{100 \mathrm{~s}} * \mathrm{X}_{10}$

(App. A, No. 52)

The flow of TRU from soil across the rumen wall is estimated as:

$\mathrm{F}(10 \cdot 13 \cdot 16 \cdot 17,14)=\mathrm{I}_{128 \mathrm{k}} * 0.001 * \mathrm{X}_{10}$

(App. A, p. 10, No. 53)

The flow of TRU into the lungs from the atmosphere is estimated from a human model:41

$\mathrm{F}(12,18)=0.15 * \mathrm{X}_{12} * \mathrm{I}_{1291}-4.34 \times 10^{-4} * \mathrm{X}_{18}$

(App. A, No. 54)

The flow of TRU from the pulmonary region of the lungs to the gut and across the rumen wall is:

$\mathrm{F}(18 \cdot 17 \cdot 16 \cdot 13,14)=1.38 \times 10^{-4 *} 0.001 * \mathrm{X}_{18}$

(App. A, pp. 10-11, No. 55)

The flow of TRU directly across the alveolar membrane is estimated as:

$\mathrm{F}(18,14)=3.61 \times 10^{-3} \times \mathrm{X}_{18}$

(App. A, No. 56)

Also, the flow of TRU across the rumen wall from nonpulmonary deposits is estimated as:

$\mathrm{F}(12 \cdot 17 \cdot 16 \cdot 13,14)=\mathrm{I}_{1291} * \mathrm{X}_{12} * 0.35 * 0.001$

(App. A, No. 57)

Finally, the flow of TRU across the rumen from consumed forage:

$\mathrm{F}(5 \cdot 7 \cdot 17 \cdot 16 \cdot 13,14)=0.001 * \mathrm{~F}(10,13 \cdot 16 \cdot 17)$

(App. A, No. 58) 
TRU flow which enters the rumen from all sources and is not assimilated is considered as activity associated with the humus compartment $\left(\mathrm{X}_{\mathrm{e}}\right)$ :

$$
\begin{aligned}
\mathrm{F}(13 \cdot 16 \cdot 17,9) & =\mathrm{F}(10 \cdot 13 \cdot 16 \cdot 17,14)+\mathrm{F}(18 \cdot 17 \cdot 16 \cdot 13,14) \\
& +\mathrm{F}(12 \cdot 17 \cdot 16 \cdot 13,14)+\mathrm{F}(5 \cdot 7 \cdot 17 \cdot 16 \cdot 13,14) /\left(0.999{ }^{*} \mathrm{I}_{122 !}{ }^{*} \mathrm{C}_{\mathbf{8 4 1}}\right.
\end{aligned}
$$

(App. A, No. 59)

TRU flow to milk is estimated as:

$$
\begin{aligned}
\mathrm{F}(14,15) & \left.=\mathrm{X}_{141}{ }^{*} \mathrm{C}_{321} * 0.001 / \mathrm{I}_{1801} * 0.9\right) & & \mathrm{i} \geq 3 \\
& =0 & & \mathrm{i}<3
\end{aligned}
$$

where

$\mathrm{I}_{1801}=\left(0.07 * \mathrm{X}_{16}\right)^{0 . \mathrm{s}}$

the flow of TRU from milk is set at:

$\mathrm{F}(15, \mathrm{P})=1$

(App. A, No. 61)

The concentrations of TRU in several tissues of the herd are estimated by weighting of concentrations in specific age groups. ${ }^{2}$ The tissues are:

1) TRU activity/animal unit in breeding herd (3-8 year olds)

2) TRU activity/animal unit in total herd

3) TRU activity/ $\mathrm{Kg}$ in muscle tissue of herd

4) TRU activity/Kg in liver tissue of herd

5) TRU activity $/ \mathrm{Kg}$ in bone tissue of herd

6) TRU activity/animal unit in newborn herd

The calculations assume that about $10 \%, 45 \%$, and $45 \%$ of the TRU reside in muscle, liver and bone, respectively. The concentration in newborn is assumed to be $1 / 1000$ of the TRU concentration in the breeding herd.

Biomass of some organs are power function estimates:42

Blood biomass $=0.07 * \mathrm{X}_{14}^{0.04}$

Liver biomass $=0.02 * \mathrm{X}_{14}{ }^{0.7}$

Bone biomass $=0.05 * \mathrm{X}_{14}$

Finally, the total biomass of muscle tissue available to the population on a yearly basis is estimated at $45 \%$ of the total mass of all ruminants in the herd adjusted for the fraction which comes from outside sources $\left.\left(I_{131}\right)\right)^{1.2}$ 


\section{PERIPHERAL FLOW THROUGH FOREST STRUCTURE AND FOREST MANAGEMENT MODUI.ES}

The development of strategies for estimating the flow of biomass and TRU through a forest ecosystem has been given special consideration in the overall model ${ }^{4,5}$ because of the importance of these ecosystems in the study area. The forest structure module does not affect the flow of biomass, but partitions it into subcompartments with differing attributes. Likewise, TRU flow is not affected in a direct sense, and is also partitioned concomitantly with biomass. Access to the forest management module will affect flow and is functionally dependent on the previous module as a driver. In addition, other attributes of a forest ecosystem are outputs of this module such as crown cover, bole diameter distribution, age estimates, and the TRU specific activity in the wood of trees with different diameters.

Miost of the strategies employed in the model are hypothetical, and are the result of module generalizations from specific attributes of a Ponderosa Pine type forest which was used in its development. ${ }^{4,8,28,43}$ The present module is the result of an earlier development ${ }^{2}$ which began by comparing diameter class evolution as a dynamic modified log-normal distribution 4 based on total volume of a stand in a given area. As a result of tuning of such a model, results were obtained which gave reasonable output, but it was not sensitive or capable of coping with perturbations of the type required for forest management operations; nor for negative biomass excursions from one year to the next. A later development ${ }^{b}$ attempted to couple a log-normal type generator for initial diameter classes, and to interphase the generator with a compartmental model strategy that would yield results similar to a modified log-normal distribution generator applied to the whole stand volume. Subsequent development led to complete replacement of distribution generators for all classes, and to the refinement of the compartmental approach which yields satisfactory forest structure operation for Ponderosa Pine ${ }^{45}$ and Pinon woodland forest."

A diagram of the forest structure module is presented in Fig. 11 and is a modification of an earlier version. ${ }^{s}$ The flow of biomass into the module is controlled by the gate $I_{\lrcorner_{10}}$ for a sprcific plant; where the following condition must be satisfied for access:

$\mathrm{L}_{101} \leq 733\left(\mathrm{I}_{132 \mathrm{~s}} / 2\right) *\left(1-0.5^{*} \mathrm{I}_{471}\right)=\mathrm{I}_{1393}$

(App. B, No. 2)

where $\left(I_{1801}\right)$ is an estimate of biomass density required before diameters larger than 3 diameter units can be expected. The latter is either a virtual or a real estimate of current biomass for a given plant type. Two virtual estimates are formed:

$\mathrm{I}_{1841}-0.142 * \mathrm{I}_{271} * \mathrm{C}_{281} *\left(1-\mathrm{I}_{471} / 2\right) /\left(\mathrm{I}_{371} * \mathrm{I}_{981}\right)-\mathrm{I}_{1881}$

(App. B, No. 84)

where

$I_{1981}=0.142 * I_{271} * C_{201} *\left(1-I_{473} / 2\right) /\left(I_{371} * I_{981}\right)$

(App. B, No. 85)

for the previous year; it is recalculated for the current year to compare with $\left(L_{101}\right)$ as described for gating into the module. The virtual volume estimates allow diameter classes to have differential dianeter growth rates for trees with different taper coefficients. The taper coefficient for Pinon Pine and Ponderosa Pine have been estimated as:

$\mathrm{C}_{2 \mathrm{O}_{\mathrm{g}}}=0.5 / 0.2=2.5 ; \mathrm{C}_{2 \mathrm{q}_{\mathrm{b}}}=0.41 / 0.3=1.36$ 

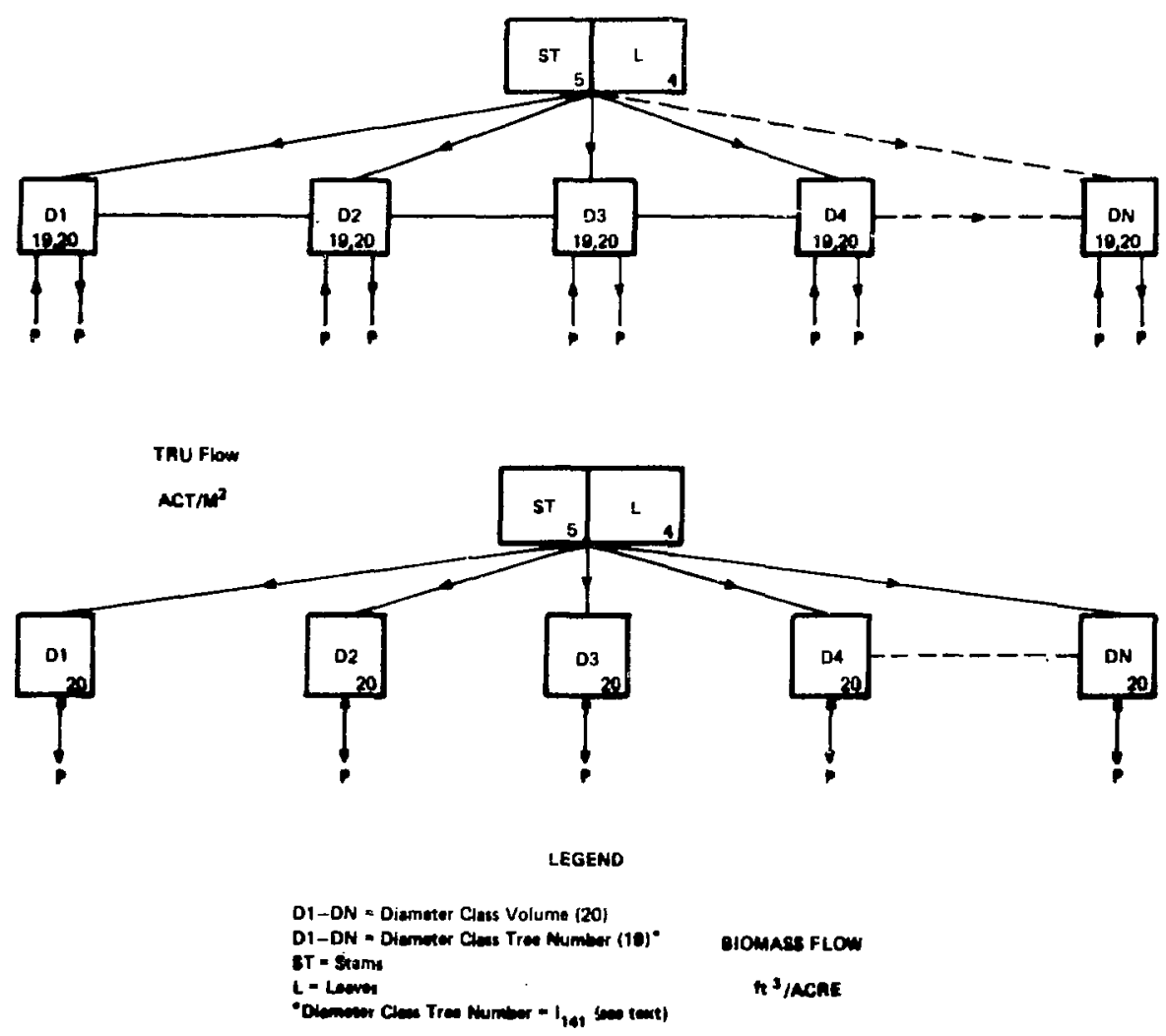

Fig. 11.

Biomass and TRU flow in forest structure module.

where

$(0.51,0.41)=$ dry wood density, $(0.2,0.3)=$ simple curvilinear regression coefficients for estimating the mass of a tree from the bole diameter for Pinon ${ }^{40}$ and for Ponderosa Pine as described later in this report. Taper coefficients for other tree and shrub types are unverified estimates since regression coefficients are not available. Also, if either succession or niche competition is accessed, the virtual estimates allow virtual tree growth, although at reduced densities as the case may be. The real volume of the stand is estimated as:

$I_{1101}=0.142 * I_{n 1}$

where

$I_{19 e 1}=$ wood volume of stand, $\mathrm{ft}^{\mathrm{s}}$

and

$I_{m 1}=$ biomass density of stand, $\mathrm{g} / \mathrm{m}^{2}$ above ground, 
The virtual volume of the smallest diameter class $\left(0-\mathrm{I}_{1821}, 1 \leq 22\right)$ or the regenerative class is a function of total above ground biomass of the stand:

$$
\begin{aligned}
\mathrm{X}_{20_{1}} & =\mathrm{I}_{1381} * \operatorname{EXP}\left(-1 / \mathrm{C}_{271}\right) *\left(\mathrm{I}_{1981}-\mathrm{I}_{1831}\right) & \mathrm{X}_{20_{1}}>\mathrm{I}_{187 \mathrm{k}} \\
& =0.0 & \mathrm{X}_{20_{1}} \leq \mathrm{I}_{197 \mathrm{k}}
\end{aligned}
$$

where

$1 \leq \mathrm{k} \leq 60$, and

$\mathrm{I}_{1 \mathrm{s7 \textrm {k }}}=\mathrm{I}_{\mathbf{1 4 4 \mathrm { k }}} * 0.3$

$\mathbf{k}=1$

$\mathrm{I}_{137 \mathrm{x}}=\mathrm{I}_{\mathrm{t} 4 \mathrm{sk}} * 77.3 / \mathrm{I}_{180 \mathrm{t}}$

$1<\mathrm{k} \leq 60$

(App. B, No. 86)

This class regenerates a forest structure at low biomass densities, and gradually declines in volume and importance as biomass density accumulates; or until another regenerative event is simulated (forest fire, harvesting, etc.) which acts to lower the above ground biomass density. The regenerative class $(k=1)$ is directly coupled to the succeeding diameter class:

$\mathrm{I}_{182_{2}}=\mathrm{k} * \mathrm{C}_{\mathrm{as1}}, \mathrm{k}=2$

(App. B, No. 83)

as are all other diameter classes $(k \leq 60)$ to each other. This allows proper coupling of the regenerative class volume $(\mathrm{k}=1)$ to the second volume class $(\mathrm{k}=2)$ :

$\mathrm{X}_{20_{2}}=\mathrm{I}_{1881}-\mathrm{X}_{20_{1}}$

(App. B, No. 7)

Succeeding intraclass transports depend on competition factors acquired by different diameter classes:

1) bole diameter area

2) crown diameter area

3) number of trees in class

A competition factor is estimated as follows:

$I_{1881}=I_{140 k} *\left(I_{1891} * I_{1411}+C_{201} / 2\right)^{2} *\left(I_{1891}\right)^{2}$

(App. B, No. 87)

This factor is used to partition biomass into smaller volume parcels which are used as allocation flows:

$$
\mathrm{I}_{142 \mathrm{k}}=\mathrm{I}_{1841} * \mathrm{I}_{18 \mathrm{~kg}} / \sum_{\mathrm{k}=1}^{\mathrm{n}} \mathrm{I}_{188 \mathrm{k}}, \quad 2 \leq \mathrm{n} \leq 60 \quad \text { (App. B, No. 89) }
$$

These parcels are then used to simulate interflow and intraflow estimates between diameter classes above the first. The intraflow strategy is modeled to make the parcel generated by the previous class diameter dependent on net wood production: 


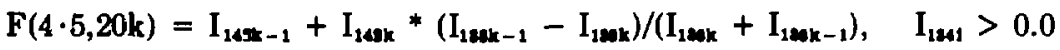

$$
\begin{aligned}
& =-\mathrm{I}_{1 \mathrm{eak},}, \quad \mathrm{I}_{12 \mathrm{sk}} \leq 0.0, \mathrm{I}_{12 \mathrm{~A} 1}>0.0
\end{aligned}
$$

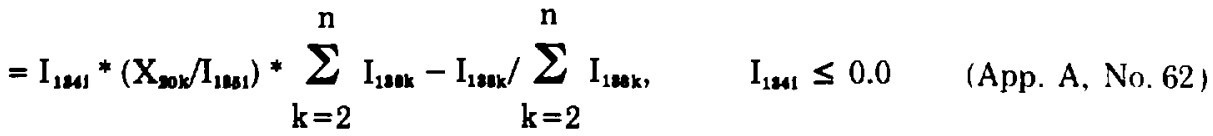

The three processes describe intraflow as a transport from a previous class if net production is positive. However, if net production is negative, a net transport of wood out of a class is modeled if the previous class is empty; otherwise a decrease inversely proportional to the competition factor $\left(\mathrm{I}_{12 u}\right)$ is established.

Interclass transport occurs only if net wood production is positive:

$\mathrm{F}(20 \mathrm{k}, 20 \mathrm{k}+1)=\mathrm{I}_{1491} *\left(\mathrm{I}_{1 \mathrm{100k}-1}\right) /\left(\mathrm{I}_{1 \mathrm{1ak}}+\mathrm{I}_{100 \mathrm{k}-1}\right), \quad 2 \leq \mathrm{k} \leq 60 . \quad$ (App. A, No. 63)

This flow is of either sign and dependent on relative values of two succeeding competition factors. A flow of this type must be adjusted for materials balance to be described later in this report.

Mortality flows are made dependent on competition factors, total plant crown cover, and wood turnover rate:

$$
\mathrm{F}(20 \mathrm{k}, \mathrm{P})=\mathrm{X}_{20 \mathrm{k}} * \mathrm{I}_{471} /\left(\left(\mathrm{C}_{271} *\left(\sum_{\mathrm{k}=1}^{n} \mathrm{I}_{1 \mathrm{agk}}\right)-\mathrm{I}_{19 \mathrm{gsk}}\right) / \sum_{\mathrm{k}=1}^{n} \mathrm{I}_{198 \mathrm{k}}\right) \text {, }
$$

$$
2 \leq n \leq 60
$$

(App. A, No. 64)

This flow is adjusted for materials balance as must be the condition:

$$
\begin{aligned}
X_{20 k} & =X_{20 k} & & I_{187 k}>I_{144 k} * 77.3 / I_{1801} \\
& =0 & I_{1871} \leq I_{140 k} * 77.3 / I_{1801} & \text { (App. A, No. 65) }
\end{aligned}
$$

which does not allow a new diameter class to be created until an estimated minimum wood volume has been attained. The volume increment difference is estimated as:

$\mathrm{I}_{149 \mathrm{i}}=\mathrm{I}_{18 \mathrm{at}}-\sum_{\mathrm{k}=1}^{\mathrm{n}} \mathrm{X}_{\underline{20 \mathrm{k}}}$

The number of trees per acre is estimated as:

$\mathrm{X}_{101}=\mathrm{X}_{\mathbf{2 0 k}} / \mathrm{I}_{14 \mathrm{k}}$

(App. A, No. 66) 
The adjusted flow into each diameter class $(k>1)$ is estimated as:

$$
\begin{aligned}
F(P, 20 k) & =I_{1491} * I_{188 j} / \sum_{k=2}^{n} I_{138 k} & I_{1431}>0.0 \\
& =I_{1431} *\left(1 / I_{188 k}\right) / \sum_{k=2}^{n} 1 / I_{188 k} & I_{1431} \leq 0.0
\end{aligned}
$$

The final adjustment on volume for all classes is:

$$
\mathrm{X}_{20 \mathrm{~K}}=\mathrm{X}_{20 \mathrm{k}} * \mathrm{I}_{18 \mathrm{~s} /} / \mathrm{I}_{\mathrm{1ss} \mathrm{I}}
$$

The number of trees/acre in each diameter class is determined first by estimating the volume of a tree in a given diameter class:

$$
\begin{aligned}
& \mathrm{I}_{144 \mathrm{k}}=0.3 * 0.035 *\left(\mathrm{I}_{1 \mathrm{483}} / \mathrm{C}_{201}\right) *(2 * 24 * 2.54)^{2} \quad \mathrm{k}=1 \\
& =(0.035) *\left(\mathrm{I}_{1881} / \mathrm{C}_{201}\right) *\left[\left(\mathrm{k} *\left(\mathrm{I}_{1821}-1 / 2\right)\right)^{2}+\left(\mathrm{k} *\left(\mathrm{I}_{1991}+1 / 2\right)\right)^{2}\right] / 2 \quad 2 \leq \mathrm{k} \leq 60
\end{aligned}
$$

(App. B, No. 91)

Volume estimates of this type have been obtained for pinon and juniper, ${ }^{46}$ and for ponderosa pine during the course of this research. They are based on curvilinear regression analysis of tree dry weight versus bole diameter.

Weight $(\mathrm{kg})=b_{1} D^{2}$, where $b_{2}=$ regression coefficient, and $D=$ bole diameter in $\mathrm{cm}$.

More information of this type is required for other trees included within the model. The number of trees/acre with each class diameter is estimated as:

$\mathrm{I}_{140 \mathrm{k}}=\mathrm{X}_{\mathrm{gak}} / \mathrm{I}_{14 \mathrm{k}}$

The flow of TRU to a given diameter class is modeled after two events:

1) addition of TRU during current year

2) loss or gain of TRU through volume increment

The addition of TRU during the current year is estimated as:

$\mathrm{F}(5 \cdot 4,20 \mathrm{k})=\left|\mathrm{I}_{1 \mathrm{Bal}}\right| * \mathrm{I}_{1 \mathrm{es1}} *\left(\mathrm{I}_{1 \mathrm{Bed}} / \mathrm{I}_{2 \mathrm{se1}}\right)$

(App. A, No. 68)

which allows activity to enter or leave a diameter class during positive and negative excursions of wood production.

Further additions or removal of TRU from a diameter class depends on net wood production:

$$
F(P, 20 k)=\left(I_{1471} / 0.142\right) *\left(X_{10 k}\right)_{p} /\left(X_{100 K} / 0.142\right) \quad I_{1471}>0.0 \quad \text { (App. A, No. 69) }
$$


$\mathrm{F}(20 \mathrm{k}, \mathrm{P})=\left(\mathrm{I}_{1471} / 0.142\right) *\left(\mathrm{X}_{20 \mathrm{k}}\right)_{\mathrm{p}} /\left(\mathrm{X}_{20 \mathrm{k}} / 0.142\right) \quad \mathrm{I}_{1471} \leq 0.0$

where (p) indicates TRU material.

All additions described are subjected to materials balance as the flow criteria applied are proportional but not equitable to total activity in the stand. Hence, the total activity of each diameter class is adjusted:

$\mathrm{X}_{20 \mathrm{k}}=\mathrm{X}_{20 \mathrm{k}} *\left(\mathrm{I}_{1401} /\left(\mathrm{X}_{0}+\mathrm{X}_{6}\right)_{1}\right.$

where

$I_{1481}=\sum_{k=1}^{n} X_{20 k}$

(App. B, No. 92)

which represents virtual total activity of the stand prior to adjustment. The specific activity of each diameter class:

$I_{180 k}=\left(X_{20 k}\right)_{p} /\left(X_{20 k} / 0.142\right)$

(App. B, No. 93)

where (p) refers to TRU pool material. Finally, each time a succeeding diameter class is created the specific activity is set to that of the previous class:

$I_{180 k}=I_{160 k-1}$

(App. B, No. 94)

In addition to the flows described above, other attributes of the forest structure are processed in this module: ${ }^{2}$ crown cover, basal area coverage, geometric mean age-etc. Also, the number of simulated years marking the creation of a new diameter class, the interval between formation of a new class and creation of the succeeding one, and an estimate of average age based on these criteria are part of the module; they are described elsewhere. ${ }^{2}$

Access to the forest management module, unlike the forest structure module, does affect both biomass and TRU through user selected events. However, the module is actually an extension of the latter, and is functionally dependent on it. Selected harvesting schemes, ${ }^{2,28}$ have been included:

1) selected diameter cuts

2) "Reverse-J" cut

3) forest fire event

A forest fire event also includes the option of complete or partial removal of timber. About 1/10 of the TRU in the wood is assumed to enter the above ground humus compartment containing TRU $\left(\mathrm{X}_{\mathrm{g}}\right)$. Most of the operations involved in the module are "bookkeeping" operations which augment or decrease pool materials; they are described in the code documentation portion of this report. ${ }^{2}$

\section{REFERENCES}

1. A. F. Gallegos, B. J. Garcia. and C. M. Sutton, "Operating Instructions and Variable Library for TRU Biological Transport Model, 1977," (this report) Appendix H.

2. A. F. Gallegos, B. J. Garcia, and C. M. Sutton, "Code Documentation of TRU Biological Transport Model, 1977," (this report) Appendix G. 
3. "Semiannual Report. Transuranic Solid Waste Management Research Programs, JulyDecember, 1974, "Los Alamos Scientific Laboratory report, LA-6100-PR (1975).

4. "Semiannual Report. Transuranic Solid Waste Management Research Programs, JanuaryJune, 1975," Los Alamos Scientific Laboratory report, LA-6264-PR (1976).

5. "Semiannual Report. Transuranic Solid Waste Management Research Programs, JulyDecember, 1975," Los Alamos Scientific Laboratory report, LA-6481-PR (1976).

6. M. L. Wheeler, W. J. Smith, and A. F. Gallegos, "A Preliminary Evaluation of the Potential for Plutonium Release from Burial Grounds at Los Alamos Scientific Laboratories," Los Alamos Scientific Laboratory report, LA-6694-MS (1977).

7. A. F. Gallegos, M. L. Wheeler, W. J. Whitty, and W. J. Smith, "Environmental Evaluation of Solid Radioactive Waste Disposal Areas," Bulletin, New Mexico Academy of Science 15 (2) (abstract), April (1975).

8. A. F. Gallegos, and L. J. Johnson, "A Simulation Study of Mineral Recycling in Ponderosa Pine Forests," Proceedings Supplement, Jour. Acad. Sci., 11, (abstract), April (1976).

9. W. J. Smith, A. F. Gallegos, and L. J. Johnson, "LASL Models for Environmental Transport of Radionuclides," Modeling and Simulation, 8, Instrument Society of America, Pittsburgh (1977).

10a. International Commission on Radiological Protection, Task Group on Lung Dynamics, "Deposition and Retention Models for Internal Dosimetry of the Human Respiratory Tract." Health Physics 12, (2) 173 (Feb. 1966) 73.

10b. International Commission on Radiological Protection, Committee II, "Report of Committee II on Permissible Dose for Internal Radiation, "ICRP Publ. 2. (Pergamon Press, N.Y., 1959).

10c. International Commission on Radiological Protection, "The Metabolism of Compounds of Plutonium and Other Actinides, "ICRP Publ. 19. (Pergamon Press, N.Y., 1972).

10d. I. S. Eve, "A Review of the Physiology of the Gastrointestinal Tract in Relation to Radiation Dose from Radioactive Materials," Health Physics 12. (2) 131 (Feb. 1966).

11. G. Swartzman and P. Sollins, "Conifer: A Model of Carbon and Water Flow Through a Coniferous Forest Documentation," Conifer Biome Bulletin 8 (1977).

12. J. L. Baldwin, Climates of the United States, U.S. Department of Commerce, Washington, D.C. (1973).

13. The National Atlas of the United States of America, U.S. Geological Survey, Department of the Interior, Washington, D.C. (1970).

14. Yi-Fu Tuan, C. E. Everard, J. G. Widdison, and Iven Bennett, The Climate of New Mexico (State Planning Office, Santa Fe, New Mexico, 1974). 
15. B. J. Gilbert, "Range IV Grassıand Simulation Model. Regional Analysis of Grassland Environmental Systems," Federal Grant No. ESR-72-03396-A02, Colorado State Univ., Ft. Collins, CO, Report No. 17-18 (1974).

16. W. E. Hale, L. J. Reiland, and J. P. Beverage, "Characteristics of the Water Supply in New Mexico," New Mexico State Engineers Office, Santa Fe, New Mexico, Tech. Rpt. No. 31 (1965).

17. Personal Communication, M. Wheeler, Group H-8, Los Alamos Scientific Laboratory, Los Alamos, New Mexico.

18. S. W. Running, R. H. Waring, and R. A. Rydell, "Physiological Control of Water Flux in Conifers," Oecologia (Berl.) 18, 1-16 (1975).

19. H. Precht, J. Christophersen, H. Hensen, and W. Larcher, Temperature and Life. SpringerVerlag, New York (1973).

20. R. L. Edmonds, Ed., "an Initial Synthesis of Results in the Coniferous Forest Biome, 19701973," Coniferous Forest Biome, Ecosystems Analysis Studies US/IBP, Bulletin No. 5, 4(1974).

21. C. C. Grier and R. H. Waring, "Conifer Foliage Mass Related to Sapwood Area," Forest Science 20, (3) pp. 205-20602- (1974).

22. M. H. Zimmerman and C. L. Brown, Trees: Structure and Function, Springer-Verlag, New York (1974).

23. R. L. Edmonds, Ed., "an Initial Synthesis of Results in the Coniferous Forest Biome, 19701973," Coniferous Forest Biome, Ecosystems Analysis Studies US/IBP, Bulletin No. 7, 40 (1974).

24. R. L. Burgess and R. V. O'Neill, Ed., Eastern Deciduous Forest Biome Progress Report. Environmental Sciences Division Publication No. 751. Oak Ridge National Laboratory, Oak Ridge, Tennessee, September (1975).

25. P. Van Voris and R. C. Dahlman, "Floodplain Data: Ecosystem Characteristics and ${ }^{137} \mathrm{Cs}$ Concentrations in Biota and Soil, Environmental Sciences Division Publication No. 938, Oak Ridge National Laboratory, Oak Ridge, Tennessee, November (1976).

26. F. R. Larson, "Simulating Growth and Management of Ponderosa Pine Stands," Ph.D. Dissertation, Colorado State University, Ft. Collins, Colorado (1975).

27. W. G. McGinnies and J. F. Arnold, "Relative Water Requirement of Arizona Range Plants," Arizona Agricultural Experiment Station Bul. 80, 167-246 (1939).

28. R. J. Preston, Rocky Mountain Trees. Dover Publications, Inc., New York (1968). 
29. W. E. Martin, S. G. Bloom, and R. J. Yarde, "NAEG Plutonium Study Modeling Program: Plutonium Transport and Dose Estimation Model. The Dynamics of Plutonium in Desert Environments," Nevada Applied Ecology Group, USAEC, Prog. Rpt. NVO-142, pp. 338 (1974).

30. C. T. Garten, R. H. Gardner, and R. C. Dahlman, "A Linear Compartment Model of Plutonium Dynamics in a Deciduous Forest Ecosystem," Environmental Sciences Division, Oak Ridge National Laboratory, Oak Ridge, Tennessee (unpublished manuscript) (1977).

31. D. W. Wilson, "A Mathematical Model of the Transport of Cs-137 from Fallout to Milk," Ph.D. Dissertation, Colorado State University, Ft. Collins, Colorado, p. 139 (1968).

32. B. B. Hicks, "Some Micrometeorological Aspects of Pollutant Deposition Rates at a Surface," Argonne National Laboratory, Argonne, Illinois, unpublished (1974).

33. D. H. Slade, "Meteorology and Atomic Energy," USAEC report TID-24190, pp. 202-203 (1968).

34. W. H. Langham, "Plutonium Distribution as a Problem in Environmental Science," Proc. of Environ. Plutonium Symp., Los Alamos Scientific Laboratory report No. LA-4756 (1971).

35. Kozlowski, Ed., "Tree Physiology," Colloquium for Foresters. University of Wisconsin, Department of Forestry, School of Natural Resources, College of Agricultural and Life Sciences and U.S. Forest Service; March 29-30 (1973).

36. E. M. Romney, H. M. Mork, and K. H. Larson, "Persistence of Plutonium in Soil, Plants, and Small Mammals," Health Phys., 19, 487-491 (1970).

37. J. F. Sutcliffe and D. A. Baker, "Plants and Mineral Nutrition," Studies in Biology No. 48, Edward Arnold, London (1974).

38. L. A. Stoddard and A. D. Smith, Range Management, McGraw-Hill Company, New York (1955).

39. W. P. Dasman, "Deer-Livestock Forage Studies on Interstate Winter Deer Range in California," Journal of Range Management 2 (4) 206-212 (1949).

40. W. E. Martin, S. G. Bloom, and R. J. Yarde, "NAEG Plutonium Study Modeling Program: Plutonium Transport and Dose Estimation Model. The Dynamics of Plutonium in Desert Environments, "Nevada Applied Ecology Group, USAEC, Prog. Report NVO-153 (1975).

41. International commission on Radiological Protection, Task Group on Lung Dynamics, "Deposition and Retention Models for Internal Dosimetry of the Human Respiratory Tract." Health Physics 12, (2) 173 (Feb. 1966) 73.

42. S. Brady, Bioenergetics and Growth. Reinholt, New York (1945).

43. W. P. Clary, Rocky Mountain Forest and Range Experimental Station, Forest Sciences Laboratory, NAU, Flagstaff, Arizona, personal communication, 1975. 
44. R. R. Irani and C. F. Calles, Particles Size: Measurement and Application (John Wiley \& Sons, New York (1963).

45. H. E. Brown, M. B. Baker, J. J. Rogers, W. P. Clarey, J. L. Kooner, F. R. Larson, C. C. Avery, and R. E. Campbell, "Opportunities for Increasing Water Yields and Other Multiple Use Values on Ponderosa Pine Forest Lands," USDA Forest Service, Research Paper RM-129 (1974).

46. B. J. Garcia, "Selected Characteristics of Pinon Pine," Masters Thesis, New Mexico Highlands University, Las Vegas, New Mexico (1977). 


\section{APPENDIX A}

\section{FLOWS}

\section{FIGURE 6 FLOWS}

1. $F(P, 1)=F(P, 2)=F\left(D_{1}, I_{1}, S_{1}, I_{B}, S_{2}, S_{8}, I_{4}\right)$

$F(P, 1)=$ transport of water from precipitation events to soil

$\mathrm{F}(\mathrm{P}, 2)=$ transport of water from snow to soil

$\mathrm{D}_{1}=$ annual precipitation, inches

$I_{1}=$ Jan. through Dec. monthly precipitation fractions

$S_{1}=$ random number drawn from a normal distribution $(\mathrm{m}=0, \sigma=1)$

$I_{B}=$ coefficient of variation for precipitation/event of reference site

$\mathrm{S}_{2}=$ integer value of product of random no. and no. of days in a month

$\mathrm{S}_{3}=$ random number drawn from a normal distribution $(\mathrm{m}=0, \sigma=1)$

$\mathrm{I}_{4}=$ total rainfall events/month totaling more than $0.1^{\prime \prime}$

2. $F(P, 1)=D_{1} I_{1} S_{1}\left(1+I_{8} S_{g}\right)$

$$
=0
$$

$$
\begin{aligned}
& I_{5}=\left|S_{2}\right| \\
& I_{5}=\left|S_{2}\right|
\end{aligned}
$$

$F(P, 1)=$ transport of water from precipitation events to soil

$\mathrm{D}_{1}=$ annual precipitation, inches

$I_{1}=$ Jan. through Dec. monthly precipitation fractions

$S_{1}=$ random number drawn from a normal distribution $(\mathrm{m}=0, \sigma=1)$

$\mathrm{I}_{b}=$ coefficient of variation fo: precipitation/event of reference site

$S_{3}=$ random number drawn from a normal distribution $(\mathrm{m}=0, \sigma=1)$

$S_{2}=$ integer value of product of random no. and no. of days in a month

$\mathrm{l}_{\mathrm{s}}=$ day number

3. $F(2,1)=C_{1} * X_{2}$

$\mathrm{F}(2,1)=$ transport of water from snow to soil

$\mathrm{C}_{1}=$ snow melt coefficient, $1.128 \mathrm{~mm}^{\circ} \mathrm{C}^{-1}$

$\mathrm{X}_{2}=$ precipitation as snow, $\mathrm{mm}$

4. $F(1, P)=X_{1}+P-I_{15}$

$X_{1}+P>I_{16}$

$$
=0
$$

$X_{1}+P<I_{1 s}$

$\mathrm{F}(1, \mathrm{P})=$ transport of moisture from soil due to runoff

$\mathrm{X}_{1}=$ current value of soil moisture availability

$\mathrm{I}_{16}=$ holding capacity of soil, $\% \mathrm{H}_{2} \mathrm{O}$ 
5. $F(1,3 \cdot 4 \cdot 5)=I_{18}$

$$
\begin{aligned}
& =\mathrm{I}_{16} * \mathrm{X}_{1} / \mathrm{I}_{16} \\
& =0
\end{aligned}
$$

$$
\mathrm{X}_{1}>\mathrm{I}_{10}
$$

$I_{17} \leq X_{1} \leq I_{16}$

$\mathrm{X}_{1}<\mathrm{I}_{17}$

$\mathbf{F}(1,3 \cdot 4 \cdot 5)=$ transport of moisture to roots, stems, and leaves from soil, $\mathrm{mm}$

$I_{18}=$ potential evapotranspiration rate

$\mathrm{X}_{1}=$ current value of soil moisture availability

$\mathrm{I}_{10}=$ field capacity of soil, $\% \mathrm{H}_{2} \mathrm{O}$

$\mathrm{I}_{17}=$ wilting point of soil, $\% \mathrm{H}_{2} \mathrm{O}$

6. $F(P, I)=\left(1-I_{3}\right) * I_{20}$

$\mathrm{F}\left(\mathrm{P}^{\prime}, 1\right)=$ "virtual flow of moisture from lower soil depths $(>250 \mathrm{~mm})$

$I_{s}=$ soil evapotranspiration adjustment for soil depth

$I_{20}=$ adjusted evapotranspiration rate, $\mathrm{mm} / 250 \mathrm{~mm}$

\section{FIGURE 7 FLOWS}

7. $\mathrm{F}(\mathrm{P}, 5)=\mathrm{X}_{5} * \mathrm{I}_{21} * \mathrm{I}_{22} * \mathrm{I}_{23}$ $\mathrm{L}_{2}=\mathrm{I}$

$=0$

$\mathrm{L}_{2}=0$

$F(P, 5)=$ potential gross photosynthesis, $\mathrm{g} / \mathrm{m}_{2}$

$\mathrm{X}_{\mathrm{s}}=$ needle or leaf biomass, $\mathrm{kg} / \mathrm{m}^{2}$

$\mathrm{I}_{21}=$ effect of temperature, ${ }^{\circ} \mathrm{C}$, on photosynthesis for plant

$\mathrm{I}_{22}=$ effect of soil moisture on photosynthesis for plant

$I_{2 s}=$ maximum photosynthetic efficiency (grams $\left(\mathrm{CH}_{2} \mathrm{O}\right) / \mathrm{m}^{2}$ per gram photosynthetic biomass $/ \mathrm{m}^{2}$ )

$\mathrm{L}_{2}=(0$ or 1$)$, determines whether photosynthesis is occurring

8. $\mathrm{F}(3, \mathrm{P})+\mathrm{F}(4, \mathrm{P})+\mathrm{F}(5, \mathrm{P})=\left(\mathrm{X}_{\mathrm{b}}+\mathrm{C}_{11} * \mathrm{X}_{\mathrm{s}}\right) * \mathrm{C}_{12} * \mathrm{EXP}\left(\mathrm{C}_{1 \mathrm{~s}} * \mathrm{D}_{2}\right) * \mathrm{C}_{14} \quad \mathrm{~L}_{3}=1$

$$
=0
$$$$
\mathrm{L}_{3}=0
$$

$\mathrm{F}(3, \mathrm{P})+\mathrm{F}(4, \mathrm{P})+\mathrm{F}(5, \mathrm{P})=$ total plant respiration, $\mathrm{g} / \mathrm{m}^{2}$

$\mathrm{X}_{\mathrm{b}}=$ needle or leaf biomass, $\mathrm{g} / \mathrm{m}^{2}$

$\mathrm{C}_{11}=$ respiratory maintenance component, see App. C

$\mathrm{C}_{12}=$ respiratory effect 1 , see App. $\mathrm{C}$ 
$\mathrm{C}_{13}=$ respiratory effect 2 , se $\mathrm{App} . \mathrm{C}$

$\mathrm{D}_{2}=$ daily temperature, ${ }^{\circ} \mathrm{C}$

$\mathrm{C}_{14}=$ respiratory effect 3 , see $A$.pp. $\mathrm{C}$

$L_{s}=(0$ or 1$)$ determines whether respiration is occurring( 1$)$

9. $\mathrm{F}(3, \mathrm{P})=0.3 * \mathrm{X}_{\mathrm{s}} * \mathrm{C}_{12} * \operatorname{EXP}\left(\mathrm{C}_{18} * \mathrm{D}_{2}\right) * \mathrm{C}_{14}$

$F(3, P)=$ root respiration, $\mathrm{g} / \mathrm{m}^{2}$

$\mathrm{X}_{3}=\mathrm{g} / \mathrm{m}^{2}$ live root biomass

$C_{12}=$ respiratory effect 1 , see $A p p . C$

$\mathrm{C}_{13}=$ respiratory effect 2 , see App. C

$\mathrm{D}_{2}=$ daily temperature, ${ }^{\circ} \mathrm{C}$

$\mathrm{C}_{14}=$ respiratory effect 3 , see App. C

10. $F(4, P)=$ respiration of stems, $g / \mathrm{m}^{2}$

11. $\mathrm{F}(5, \mathrm{P})=$ respiration of leaves, $\mathrm{g} / \mathrm{m}^{2}$

12. $F(P, 5)=$ potential gross photosynthesis, $g / \mathrm{m}^{2}$

13. $F(4,3)=C_{18} * I_{3 s}$

$$
=I_{36} *\left(X_{8} /\left(X_{8}+I_{27}\right)\right)
$$

$\mathrm{I}_{\mathbf{3 s}}<0$

$\mathrm{F}(4,3)=$ transport of photosynthate to roots from above ground parts, $\mathrm{g} / \mathrm{m}^{2}$

$\mathrm{C}_{18}=$ fraction of photosynthate transferred to roots (RTRANS), see App. C

$\mathrm{I}_{\mathrm{as}}=\mathrm{g} / \mathrm{cm}^{2}$, biomass net production on a given day

$\mathrm{X}_{5}=$ needle or leaf biomass, $\mathrm{g} / \mathrm{m}^{2}$

14. $F(4,7)+F(5,7)$

$$
\begin{aligned}
& =0.693 * \mathrm{X}_{4} / 730+\mathrm{I}_{27} * \mathrm{I}_{89} \\
& =\mathrm{I}_{80} * \mathrm{I}_{27} \\
& =\mathrm{I}_{88} * \mathrm{I}_{27}+\mathrm{X}_{8} \\
& =0.005^{*} \mathrm{I}_{27} \\
& =\mathrm{I}_{27} *\left(\mathrm{C}_{20} *\left(\mathrm{D}_{2}-\mathrm{C}_{21}\right)+\mathrm{C}_{22}\right) * 0.05 \\
& =\mathrm{I}_{27}^{*}\left(\mathrm{C}_{28}-\mathrm{C}_{24} * \mathrm{D}_{2}\right) * 0.05 \\
& =0.1 * \mathrm{I}_{27}
\end{aligned}
$$

$$
\begin{aligned}
& \mathrm{O}_{6}=0, \mathrm{~L}_{6}=0, \mathrm{~L}_{6}=1 \\
& \mathrm{O}_{8}=0, \mathrm{~L}_{6}=0, \mathrm{~L}_{4}=0 \\
& \mathrm{O}_{6}=0, \mathrm{~L}_{6}=0, \mathrm{~L}_{4}=0, \mathrm{D}_{2}=\mathrm{I}_{39} \\
& \mathrm{O}_{8}=0, \mathrm{~L}_{6}=1, \mathrm{~L}_{4}=0, \mathrm{C}_{26} \leq \mathrm{D}_{2} \leq \mathrm{C}_{21} \\
& \mathrm{O}_{6}=0, \mathrm{~L}_{8}=1, \mathrm{~L}_{4}=0, \mathrm{D}_{2}>\mathrm{C}_{21} \\
& \mathrm{O}_{6}=0, \mathrm{~L}_{8}=1, \mathrm{~L}_{4}=0, \mathrm{D}_{2}<\mathrm{C}_{24} \\
& \mathrm{O}_{6}=1, \mathrm{~L}_{8}=1, \mathrm{~L}_{4}=0, \mathrm{I}_{8}=\mathrm{I}_{34}
\end{aligned}
$$

$\mathrm{F}(4,7)+\mathrm{F}(5,7)=$ combined flow of stem mortality and leaf drop, $\mathrm{g} / \mathrm{m}^{2}$

$\mathbf{X}_{\varepsilon}=$ needle or leaf biomass, $\mathrm{g} / \mathrm{m}^{2}$

$I_{27}=\mathrm{g} / \mathrm{m}^{2}$ live above ground biomass

$I_{3 s}=$ factor to determine mortality of certain plant types

$\mathrm{D}_{2}=$ daily temperature, ${ }^{\circ} \mathrm{C}$

$\mathrm{C}_{20}=$ mortality factor 1 , see App. C

$\mathrm{C}_{21}=$ mortality temperature effect, ${ }^{\circ} \mathrm{C}$, see App. $\mathrm{C}$

$\mathrm{C}_{22}=$ mortality factor 2, see App. $\mathrm{C}$

$\mathrm{C}_{28}=$ mortality factor 3 , see App. C

$\mathrm{C}_{24}=$ mortality factor 4 , see App. C

$\mathrm{O}_{\mathrm{n}}=$ determines whether irrigation schedule for plant type will be part of input 
$\mathrm{L}_{\delta}=$ distinguishes woody plants $(0)$ from herb types(1)

$\mathrm{L}_{4}=$ determines whether leaf fall occurs at the end of frost-free period $(0)$ or if evergreen(1)

$\mathrm{I}_{\mathbf{3 2}}=$ end of frost-free period, day number

$\mathrm{C}_{20}=$ mortality temperature factor, lower, ${ }^{\circ} \mathrm{C}$, see App. $\mathrm{C}$

$\mathrm{I}_{s}=$ day number

$I_{39}=$ harvesting schedule, day of year

15. $\mathrm{F}(3,6)=(\mathrm{F}(4,7)+\mathrm{F}(5,7)) *\left(\mathrm{X}_{8} / \mathrm{I}_{27}\right)$

plants $(1,2,4,5,6,9,10,12,14,15,16,20,21)$

$$
=(F(4,7)+F(5,7)) *\left(X_{s} / I_{27}\right)+I_{s b} * X_{s} \quad \text { plants }(7,19)
$$

$=\mathrm{X}_{3}^{*} \mathrm{I}_{9 y}$

plants $(3,8,11,13,18)$

$=\mathrm{X}_{3}$

plants $(1,2,12), I_{s}=I_{s p}$

$\mathbf{F}(3,6)=$ mortality losses, roots to dead roots, $\mathrm{g} / \mathrm{m}^{2}$

$\mathrm{X}_{\mathrm{s}}=\mathrm{g} / \mathrm{m}^{2}$ live root biomass

$\mathrm{I}_{27}=\mathrm{g} / \mathrm{m}^{2}$ live above ground biomass

$I_{s a}=$ factor to determine mortaiity factor of certain plant types

$\mathrm{I}_{\mathrm{s}}=$ day number

$\mathrm{I}_{39}=$ harvesting schedule, day of year

$F(4,7)+F(5,7)=$ combined flow of stem mortality and leaf drop

16. $F(7,9)=I_{40} * I_{41} * X$,

$\mathrm{F}(7,9)=$ mortality losses from dead leaf stem to leaf stem litter, $\mathrm{g} / \mathrm{m}^{2}$

$\mathrm{I}_{40}=$ dead biomass temperature decay factor

$I_{\mathbf{4}}=$ dead biomass soil moisture decay factor

$\mathrm{X}_{7}=\mathrm{g} / \mathrm{m}^{2}$ dead standing biomass for specific plant

17. $F(6,8)=I_{40} * I_{41} * X_{0}$

$\mathrm{F}(6,8)=$ mortality losses from dead roots to root litter, $\mathrm{g} / \mathrm{m}^{2}$

$\mathrm{I}_{\mathbf{4 0}}=$ dead biomass temperature decay factor

$\mathrm{I}_{41}=$ dead biomass soil moisture decay factor

$\mathrm{X}_{\mathrm{b}}=\mathrm{g} / \mathrm{m}^{2}$ dead root biomass

18. $\mathrm{F}(8, \mathrm{P})=\mathrm{X}_{\mathrm{a}} * 0.3^{*} \mathrm{C}_{12} * \operatorname{EXP}\left(\mathrm{C}_{18} * \mathrm{D}_{2}\right) * \mathrm{C}_{14}$

$\mathrm{F}(8, \mathrm{P})=$ root decomposition rate, $\mathrm{g} / \mathrm{m}^{2}$

$\mathrm{X}_{\mathrm{a}}=\mathrm{g} / \mathrm{m}^{2}$ of root litter-humus

$\mathrm{C}_{12}=$ respiratory effect $1_{\text {, }}$ see App. C

$\mathrm{C}_{19}=$ respiratory effect 2 , see App. C

$\mathrm{D}_{2}=$ daily temperature, ${ }^{\circ} \mathrm{C}$

$\mathrm{C}_{14}=$ respiratory effect 3 , see App. C 
19. $\mathrm{F}(9, \mathrm{P})=\mathrm{X}_{9} * 0.3 * \mathrm{C}_{12} * \operatorname{EXP}\left(\mathrm{C}_{13} * \mathrm{D}_{2}\right) * \mathrm{C}_{14}$

$\mathrm{F}(9, \mathrm{P})=$ above ground humus decomposition rate, $\mathrm{g} / \mathrm{m}^{2}$

$\mathrm{X}_{9}=\mathrm{g} / \mathrm{m}^{2}$ above ground litter-humus

$\mathrm{C}_{12}=$ respiratory effect, see Anp. $\mathrm{C}$

$\mathrm{C}_{13}=$ respiratory effect 2 , see App. C

$\mathrm{D}_{2}=$ daily temperature, ${ }^{\circ} \mathrm{C}$

$\mathrm{C}_{14}=$ respiratory effect 3 , App. C

\section{II. FIGURE 8 FLOWS}

20. $F(P, 12)=I_{43}$

$$
\mathrm{I}_{44}
$$

$I_{48}$
$\mathrm{L}_{0}>0$

$\mathrm{L}_{7}>0$

$\mathrm{O}_{4}=1, \mathrm{O}_{\mathrm{s}}>1, \mathrm{O}_{\mathbf{4}}=3$

$\mathrm{F}(\mathrm{P}, 12)=$ llow of TRU to atmosphere, activity $/ \mathrm{m}^{\mathrm{s}}$

$\mathrm{I}_{43}=$ amount of atmospheric activity $/ \mathrm{m}^{8}$ on a given day for given year (64 day event possible, 16 years possible)

$\mathrm{I}_{44}=$ function Airac, see App. E

$\mathrm{I}_{\mathbf{4 6}}=$ activity $/ \mathrm{m}^{3}$ from fire increment to total air activity

$\mathrm{L}_{\theta}=$ total number of years of an acute event

$\mathrm{L}_{7}=$ year of initial contaminating chronic event

$\mathrm{O}_{3}=$ selector for specific plants to be simulated

$\mathrm{O}_{4}=$ number of managed cuttings or events simulated

21. $F(12, P)=X_{12}=F(P, 12)$

$\mathrm{X}_{12}=$ activity $/ \mathrm{m}^{\mathrm{a}}$ including fire increment to total air activity if simulated

22. $\left.F(10,12)=i 3 \times 10^{4}\right) *\left(I_{42} * X_{10}\right)$

$$
\mathrm{I}_{\mathbf{4 8}}=0
$$

$$
=0
$$

$$
I_{48}>0
$$

$\mathrm{F}(10,12)=$ flow of TRU from soil surface (upper $2 \mathrm{~cm}$ ) to atmosphere, activity $/ \mathrm{m}^{s}$ $\mathrm{I}_{\mathbf{1 2}}=$ resuspension factor, day

$\mathrm{X}_{10}=$ soil activity, activity/gram dry weight in a given soil horizon

$\mathrm{I}_{\mathbf{4 8}}=$ daily precipitation events

23. $F(11,12)=I_{42} * I_{11}$

$$
=0
$$

$$
I_{48}=0
$$

$$
\mathrm{I}_{43}>0
$$

$\mathrm{F}(11,12)=$ resuspension of TRU from plant surfaces, activity $/ \mathrm{m}^{3}$

$\mathrm{I}_{\mathbf{4} 2}=$ resuspension factor, day ${ }^{-1}$

$\mathrm{X}_{11}=$ plant surface activity, activity $/ \mathrm{m}^{2}$

$\mathrm{I}_{4 \mathrm{~B}}=$ daily precipitation events 
24. $F(12,10)=0.0021 * X_{12} / 3 \times 10^{4}$

$I_{40}=0$

$=0.7 * \mathrm{X}_{1 \mathrm{~g}} *\left(1-\operatorname{EXP}\left(-0.6518 * \mathrm{I}_{48}\right)\right) /\left(3 \times 10^{4}\right)$

$\mathrm{I}_{\text {4. }}>0$

$F(12,10)=$ depositional flow of TRU from atmosphere to soil, activity $/ \mathrm{m}^{2}$

$\mathrm{X}_{12}=$ activity $/ \mathrm{m}^{\circ}$ including fire increment to total air activity if simulated

$I_{4 s}=$ daily precipitation events

25. $\mathrm{F}(12,11)=0.0021 * \mathrm{X}_{12} * \mathrm{I}_{40} * 0.67$

$I_{4 s}=0$

$$
=0.7 * \mathrm{X}_{12} *\left(1-\operatorname{EXP}\left(-0.6518 * \mathrm{I}_{40}\right)\right) * \mathrm{I}_{49}
$$

$\mathrm{I}_{48}>0$

$\mathrm{F}(12,11)=$ deposition of particulates containing TRU on plant surfaces, activity $/ \mathrm{m}^{2}$

$\mathrm{X}_{12}=$ activity $/ \mathrm{m}^{2}$ including fire increment to total air activity when simulated

$I_{48}=$ daily precipitation events, $\mathrm{mm}$

$\mathrm{I}_{40}=$ leaf area index, $\mathrm{m}^{2} / \mathrm{m}^{2}$, for specific plant category

26. $F(11,10)=(0.693 / 30) * X_{11} / 3 \times 10^{4}$

$=0.3 * \mathrm{X}_{11}^{*}\left(1-\operatorname{EXP}\left(-0.6518 * \mathrm{I}_{48}\right)\right) / 3 \times 10^{4}$

$\mathrm{I}_{46}>0$

$F(11,10)=$ flow of TRU from plant to soil surfaces or weshoff, activity $/ \mathrm{m}^{2}$

$\mathrm{X}_{11}=$ activity $/ \mathrm{m}^{2}$ on leaf surface of specific plant

$I_{4 s}=$ daily precipitation events

27. $F(9,12)=I_{42} * X_{0}$

$I_{48}=0$

$=0$

$\mathrm{I}_{\mathbf{4 8}}>0$

$\mathrm{F}(9,12)=$ flow of fine litter particles containing TRU to resuspendable material, activity $/ \mathrm{m}^{2}$

$I_{42}=$ resuspension factor, day ${ }^{-1}$

$\mathrm{X}_{\mathrm{g}}=$ activity $/ \mathrm{m}^{2}$ above ground litter-humus

$I_{48}=$ daily precipitation events

28. $\mathrm{F}(9,10)=0.3 * \mathrm{X}_{0} * \mathrm{C}_{12} * \operatorname{EXP}\left(\mathrm{C}_{1 \mathrm{~s}} * \mathrm{D}_{2}\right) * \mathrm{C}_{1} / 3 \times 10^{4}$

$F(9,10)=$ flow of TRU from fine litter-humus compartment into soil surface, act $/ \mathrm{m}^{2}$

$\mathrm{X}_{9}=$ activity $/ \mathrm{m}^{2}$ above ground litter-humus

$\mathrm{C}_{12}=$ respiratory effect 1 , see App. $\mathrm{C}$

$\mathrm{C}_{1 \mathrm{~s}}=$ respiratory effect 2 , see App. C

$\mathrm{D}_{2}=$ daily temperature, ${ }^{\circ} \mathrm{C}$

$\mathrm{C}_{14}=$ respiratory effect 3 , see App. $\mathrm{C}$

29. $F(10,1)=(0.693 / 700) * X_{10}^{*}\left(3 \times 10^{4} / 3.75 \times 10^{5}\right)$

$I_{68}=0$

$=0.01 \times\left(1-\operatorname{EXP}\left(-0.6518 * \mathrm{I}_{48}\right)\right) * \mathrm{X}_{10} *\left(3 \times 10^{4 / 3.75} \times 10^{5}\right)$

$\mathrm{I}_{\mathbf{4 B}}>0$

$F(10 ; 1)=$ flow of TRU from the resuspendable horizon to the next $250 \mathrm{~mm}$ soil horizon below, activity $/ \mathrm{m}^{2}$

$\mathrm{X}_{10}=$ soil activity, activity/gram dry weight in given soil horizon

$I_{40}=$ daily precipitation events 
30. $F(1,3)=\sum_{i=1}^{I_{54}} I_{61,1} * I_{62,1} * I_{s 8,1} * X_{s} * I_{30} * 10^{\circ}$

$\mathbf{F}(1,3)=$ flow of TRU to roots from soil, activity $/ \mathrm{m}^{2}$

$I_{64}=$ number of soil horizons in $250 \mathrm{~mm}$ intervals

$I_{51}=$ soil activity, activity/gram dry weight in given soil horizon

$I_{52}=$ uptake coefficient for a radionuclide in given soil horizon

$I_{59}=$ root fraction in given soil horizon

$\mathrm{X}_{5}=$ needle or leaf biomass, $\mathrm{g} / \mathrm{m}^{2}$

$\mathrm{I}_{20}=$ adjusted evapotranspiration rate, $\mathrm{mm} / 250 \mathrm{~mm}$

$I_{b s}=$ root penetration, $\mathrm{mm}$, as a function of total days elapsed

31. $F(3,6)=X_{s}^{*}\left(F(3,6) / X_{a}\right)_{b}$

$F(3,6)=$ mortality flow of TRU from roots to dead roots, activity $/ \mathrm{m}^{2}$

$\mathrm{X}_{3}=$ activity $/ \mathrm{m}^{2}$ live root biomass

$\mathrm{X}_{8}=$ activity $/ \mathrm{m}^{2}$ dead root biomass

$\mathbf{b}^{\mathfrak{b}}=$ biomass flow

32. $\mathrm{F}(6,8)=\mathrm{X}_{6} * \mathrm{I}_{60} * \mathrm{I}_{41}$

$F(6,8)=$ flow of TRU from dead roots to humus, activity $/ \mathrm{m}^{2}$

$\mathrm{X}_{\mathrm{B}}=$ activity $/ \mathrm{m}^{2}$ dead root biomass

$\mathrm{I}_{40}=$ dead biomass temperature decay factor

$I_{41}=$ dead biomass soil moisture decay factor

33. $\mathrm{F}\left(8, \mathrm{X}_{11}\right)=\mathrm{X}_{8} * \mathrm{I}_{32,1} * 3 * \mathrm{C}_{12} * \operatorname{EXP}\left(\mathrm{C}_{1 \mathrm{~s}} * \mathrm{D}_{2}\right) * \mathrm{C}_{14}$

$F\left(8, X_{11}\right)=$ flow of TRU from root humus to soil, activity $/ \mathrm{m}^{2}$

$\mathrm{X}_{8}=$ activity $/ \mathrm{m}^{2}$ root litter-humus

$I_{62}=$ uptake coefficient for a radionuclide in a given soil horizon

$\mathrm{C}_{12}=$ respiratory effect 1 , see $A$ pp. $C$

$\mathrm{C}_{1 \mathrm{~s}}=$ respiratory effect 2 , see App. $\mathrm{C}$

$\mathrm{D}_{2}=$ daily temperature, ${ }^{\circ} \mathrm{C}$

$\mathrm{C}_{14}=$ respiratory effect 3 , see App. C

$I_{64}=$ number of soil horizons in $250 \mathrm{~mm}$ intervals

$I_{B b}=$ root penetration, $\mathrm{mm}$, as a function of total days elapsed

34. $F(3,4 \cdot 5)=X_{8} * I_{B 8} * I_{21} / I_{B 8}$

$F(3,4 \cdot 5)=$ flow of TRU from the roots into above ground parts

$\mathrm{X}_{\mathbf{3}}=$ activity $/ \mathrm{m}^{2}$ live root biomass

$I_{27}=\mathrm{g} / \mathrm{m}^{2}$ live above ground biomass

$I_{b 0}=$ biomass transpiration coefficient, day ${ }^{-1}$

$\mathrm{I}_{\mathrm{so}}=\mathrm{g} / \mathrm{m}^{2}$, above ground biomass $+\mathrm{g} / \mathrm{m}^{2}$ live root biomass for specific plant 
35. $F(4 \cdot 5,3)=\left(X_{4}+X_{8}\right) I_{68}^{*}\left(I_{60}-I_{27}\right) / I_{68}$

$\mathrm{F}(4 \cdot 5,3)=$ flow of TRU from above ground as a result of mortality losses

$\mathrm{X}_{4}+\mathrm{X}_{0}=$ activity $/ \mathrm{m}^{2}$ live above ground biomass

$\mathrm{I}_{27}=\mathrm{g} / \mathrm{m}^{3}$ live above ground biomass

$\mathrm{I}_{8 \theta}=$ biomass transpiration coefficient, day $^{-1}$

$I_{b \theta}=\mathrm{g} / \mathrm{m}^{2}$, above ground biomass $+\mathrm{g} / \mathrm{m}^{2}$ live root biomass

36. $F(4,7)+F(5,7)=\left(X_{4}+X_{b}\right)+\left((F(4,7)+F(5,7)) / I_{27}\right)_{b}$

$F(4,7)+F(5,7)=$ flow of TRU from above ground parts as a result of mortality losses

$\mathrm{X}_{1}+\mathrm{X}_{6}=$ activity $/ \mathrm{m}^{2}$ live above ground biomass

$I_{27}=g / m^{2}$ live above ground biomass

$\mathrm{b}=$ biomass flow processes

37. $F(7,9)=X_{7} * I_{40} * I_{41}$

$F(7,9)=$ flow of TRU from litter to humus, activity $/ \mathrm{m}^{2}$

$\mathrm{X}_{7}=$ activity $/ \mathrm{m}^{2}$ dead standing biomass

$I_{40}=$ dead biomass temperature decay factor

$I_{11}=$ dead biomass soil moisture decay factor

38. a) $F(4,7)+F(5,7)=0.1^{*} I_{27}$

b) $F(3,6)=X_{s}$

c) $\mathrm{I}_{27}=1.0$

d) $X_{s}=1.0$

e) $F(P, 4 \cdot 5)=0$

f) $F(4,7)+F(5,7)=0$

g) $\mathrm{I}_{27}=20.0$

$\mathrm{X}_{7}=\mathrm{X}_{7} *\left(20 / \mathrm{I}_{27}\right)$

$$
\mathrm{k}=2 ; \mathrm{I}_{\mathrm{B}}=\mathrm{I}_{\mathrm{a2,1}}
$$

$\mathrm{k}=2 ; \mathrm{I}_{661}>\mathrm{I}_{6}>\mathrm{I}_{641}$

$F(4,7)+F(5,7)=$ flow of TRU from above ground parts as a result of mortality losses, activity $/ \mathrm{m}^{2}$

$\mathrm{I}_{27}=\mathrm{g} / \mathrm{m}^{2}$ live above ground biomass

$\mathrm{F}(3,6)=$ mortality losses roots to dead roots

$\mathrm{X}_{3}=\mathrm{g} / \mathrm{m}^{2}$ live root biomass

$\mathrm{I}_{\mathrm{s}}=$ day number

$\mathrm{I}_{\mathrm{e2}}=$ harvesting schedules, day of year

$\mathrm{F}(\mathrm{P}, 4 \cdot 5)=$ gross photosynthesis for irrigated crop, $\mathrm{g} / \mathrm{m}^{2}$

$\mathrm{I}_{8 s}=$ irrigation schedules, day of year, when water is applied

$\mathrm{I}_{04}=$ harvesting schedules, day of year

$\mathrm{X}_{7}=\mathrm{g} / \mathrm{m}^{2}$ dead standing biomass

$k=$ harvesting schedule index, $2 \leq k \leq 8$ 


\section{FIGURE 10 FLOWS}

39. $\mathrm{F}(\mathrm{P}, 13)=\mathrm{I}_{81}=\left({ }_{104 \mathrm{k}, 1}-\mathrm{I}_{130, \mathrm{k}, 1}\right) *\left(1.0-0.3 * \mathrm{I}_{82,1}\right)$

$\mathrm{F}(\mathrm{P} .13)=$ amount of forage consumed by an animal unit on a given day, $\mathrm{kg} / \mathrm{day}$ $\mathrm{I}_{\mathrm{at}}=$ ingestion rate, $\mathrm{kg} / \mathrm{day}$

$\mathrm{I}_{104}=$ rumen capacity, $\mathrm{kg}$, for given age categroy of specific animal category

$I_{110}=$ rumen fill, $\mathrm{kg}$, for $\mathrm{kth}$ population age group, lth animal category

$I_{s 2,1}=$ weighted average $\%$ nitrogen content in ingesta of 1 th animal group

40. $F(13,17)=I_{108}=\left(1-I_{B 83}\right) * 0.45+I_{s s 1} * 0.7$

$\mathrm{F}(13,17)=$ digestible portion of intake, $\mathrm{kg} /$ day

$I_{105}=$ digestible fraction of ingested forage

$I_{83}=$ cumulative green biomass uptake for kth food preference category, $\mathrm{kg}$

41. $F(13,16)=I_{107}=I_{108} * I_{168}$

$\mathrm{F}(1,3,16)=$ energy derived from the digestible fraction, $\mathrm{kcal} / \mathrm{day}$

$l_{107}=$ microprotein in rumen, grams

$I_{106}=$ energy in digestible fraction of intake, kcal

$I_{169}=$ grams $/ \mathrm{kcal}$ of microprotein in digestible fraction

42. $\mathrm{F}(16,9)=\left(0.52 * \mathrm{X}_{10}\right) * 0.75$

$F(16,9)=$ flow of microprotein from the rumen to humus compartment, g/day

$\mathrm{X}_{16}=$ microprotein level, grams, in rumen for kth age, lth animal group

43. $F(13,17)=F(13,17)+I_{1091} * I_{1081} * F(13,17)$

$F(13,17)=$ net flow of digestible material, $\mathrm{kg} /$ day

$I_{108}=$ fermentation digestion rate, fraction $/ \mathrm{kg}$

$I_{100}=$ rumen exit rate, fraction/day

44. $F(17,9)=\left(I_{1091} * X_{17}\right) * 0.75$

$F(17,9)=$ exit of digestible forage portion from the rumen, $\mathrm{kg} /$ day

$\mathrm{X}_{17}=$ digestible dry matter, $\mathrm{kg} /$ day, intake rate for $\mathrm{kth}$ population group of lth animal category

$\mathrm{I}_{10 \mathrm{\theta}}=$ rumen exit rate, fraction/day

45. $\mathrm{F}(13,9)=\left(\mathrm{I}_{1091} * \mathrm{X}_{1 \mathrm{~s}}\right) * 0.75$

$\mathrm{F}(13,9)=$ flow of undigested food from the rumen, $\mathrm{kg} /$ day

$\mathrm{I}_{109}=$ rumen exit rate, fraction/day

$\mathrm{X}_{1 \mathrm{~s}}=$ rumen capacity, $\mathrm{kg}$, for given age of specific animal category 


$$
\begin{array}{ll}
=I_{1181} / 450^{\prime} & I_{1161}=0.0 \\
=I_{1181} /\left(I_{1171} * 4300\right) & 0 \leq I_{1171}>1.0
\end{array}
$$

$\mathrm{F}(13 \cdot 16 \cdot 17,14)=$ actual weight gain by an animal unit on a daily basis, $\mathrm{kg} /$ day $I_{116}=$ net available energy, $\mathrm{kcal}$

$I_{117}=$ fraction of available energy for growth, kcal

47. $\mathrm{F}(4 \cdot 5,13 \cdot 16 \cdot 17)=\mathrm{I}_{\mathrm{sgk}} *\left(\mathrm{I}_{1231} / \mathrm{I}_{7 \mathrm{~mm}}\right) *\left(\mathrm{I}_{\mathrm{som}} / \mathrm{I}_{\mathrm{sok}}\right) * \mathrm{I}_{121 \mathrm{k}}$

$F(4 \cdot 5,16 \cdot 16 \cdot 17)=$ amount of biomass removed from each plant category by animal unit, $\mathrm{g} /$ day

$I_{80}=$ uptake, $\mathrm{kg} /$ day, for ith preference category

$I_{B 8}=$ cumulative green biomass uptake for kth food preference category

$I_{72}=$ available forage, $\mathrm{g} / \mathrm{m}^{2}$, from all forage sources of ith preference category

$I_{121}=$ fraction of area grazed by an age group of given animal population

$I_{12 s}=\mathrm{g} / \mathrm{m}^{2}$ of green and dry standing biomass

48. $F(7,13 \cdot 16 \cdot 17)=\left(1-I_{\text {bok }}\right) *\left(I_{128 /} / I_{72 m}\right) *\left(I_{80 m m} / I_{80 k}\right) * I_{121 k}$

$\mathrm{F}(7,13 \cdot 16 \cdot 17)=$ amount of biomass uptake from dead standing biomass, $\mathrm{g} / \mathrm{m}^{2}$

$I_{38}=$ cumulative green biomass uptake for kth food preference category

$\mathrm{I}_{128}=\mathrm{g} / \mathrm{m}^{2}$ of green and dry standing biomass

$I_{72}=$ available forage, $\mathrm{g} / \mathrm{m}^{2}$, from all forage sources of ith preference category

$I_{\mathbf{s}_{0}}=$ uptake, $\mathrm{kg} /$ day, for ith preference category

$I_{121}=$ fraction of area grazed by an age group of given animal population

49. $F(4 \cdot 5,13 \cdot 16 \cdot 17)=\left(X_{4}+X_{6}\right) *(F(4 \cdot 5,13 \cdot 16 \cdot 17))_{6} I_{27}$

$\mathrm{F}(4 \cdot 5,13 \cdot 16 \cdot 17)=$ TRU flow from green biomass into the rumen, activity/day

$F(4 \cdot 5,13 \cdot 16 \cdot 17)=$ amount of bion.ass removed from each plant category by animal unit, $g /$ day

$\mathrm{X}_{4}+\mathrm{X}_{8}=$ activity $/ \mathrm{m}^{2}$ live above ground biomass

$\mathrm{I}_{27}=\mathrm{g} / \mathrm{m}^{2}$ live above ground biomass

$\mathrm{b}=$ biomass flow

50. $F(7,13 \cdot 16 \cdot 17)=X_{7}^{*}\left(F(7,13 \cdot 16 \cdot 17) / X_{7}\right)_{b}$

$\mathrm{F}(7,13 \cdot 16 \cdot 17)=$ TRU flow from dry biomass to rumen, activity $/ \mathrm{m}^{2}$

$\mathrm{X}_{7}=$ activity $/ \mathrm{m}^{2}$ of dead standing biomass

$\mathrm{b}=$ biomass flow 
51. $F(11,13 \cdot 16 \cdot 17)=X_{11} *\left[F(7,13 \cdot 15 \cdot 17)+F(4 \cdot 5,13 \cdot 16 \cdot 17) /\left(I_{27}+X_{7}\right)\right]_{b}$

$\mathrm{F}(11,13 \cdot 16 \cdot 17)=$ TRU flow from dry biomass surface to rumen, activity/day

$\mathrm{F}(7,13 \cdot 16 \cdot 17)=$ flow from dry biomass to rumen, $\mathrm{g} / \mathrm{m}^{2}$

$\mathrm{F}(4 \cdot 5,13 \cdot 16 \cdot 17)=$ flow from green biomass in to the rumen, $\mathrm{g} / \mathrm{m}^{2}$

$\mathrm{X}_{11}=$ activity $/ \mathrm{m}^{2}$ on plant surfaces

$\mathrm{I}_{27}=\mathrm{g} / \mathrm{m}^{2}$ live above ground biomass

$\mathrm{X}_{\mathrm{T}}=$ activity $/ \mathrm{m}^{2}$ of dead standing biomass

$\mathrm{b}=$ biumass flow

52. $F(10,13 \cdot 16 \cdot 17)=I_{128} * C_{32 k} * I_{100} * X_{10}$

$F(10,13 \cdot 16 \cdot 17)=$ flow of TRU from soil into the rumen, activity/day

$I_{128}=$ soil ingestion, grams/day

$\mathrm{C}_{32}=$ fraction of population by age $(Z)$, see $A$ pp. C

$\mathbf{J}_{100}=$ number of animal units on range for a given grazing interval

$\mathrm{X}_{10}=$ soil activity, activity/gram dry weight in top $2 \mathrm{~cm}$ soil horizon

53. $F(10 \cdot 13 \cdot 16 \cdot 17,14)=I_{128 \mathrm{k}} * 0.001 * \mathrm{X}_{10}$

$F(10 \cdot 13 \cdot 16 \cdot 17,14)=$ flow of TRU from soil sources across rumen wall, activity/day

$\mathrm{I}_{120}=$ soil ingestion, $\mathrm{g} /$ day

$\mathrm{X}_{10}=$ soil activity, activity/gram dry weight in top $2 \mathrm{~cm}$ soil horizon

54. $F(12,18)=0.15 * \mathrm{X}_{12} * \mathrm{I}_{12 \mathrm{oh}}-4.34 \times 10^{-4 *} \mathrm{X}_{18}$

$\mathrm{F}(12,18)=$ flow of TRU into the lungs from atmosphere, activity/day

$\mathrm{X}_{12}=$ activity $/ \mathrm{m}^{8}$ including fire increment to total air activity if simulated

$\mathrm{I}_{128}=$ inhalation rate, $\mathrm{m}^{8} / \mathrm{day}$, for $\mathrm{kth}$ population category, lth animal category

$\mathrm{X}_{10}=$ accumulated activity entering pulmonary region for $k$ th population category, 1 th animal category

55. $F(18 \cdot 16 \cdot 17 \cdot 13,14)=1.34 \times 10^{-4 * 0.001 * X_{18}}$

$F(18 \cdot 16 \cdot 17 \cdot 13,14)=$ flow of TRU from pulmonary region of lungs to the gut and across the rumen wall, activity/day

$\mathrm{X}_{10}=$ accumulated activity entering pulmonary region for lth animal category

56. $F(18,14)=3.61 \times 10^{-*} * \mathrm{X}_{10}$

$\mathrm{F}(18,14)=$ flow of TRU directly across the alveolar membrane, activity/day

$\mathrm{X}_{18}=$ accumulated activity entering pulmonary region for Ith animal category

57. $\mathrm{F}(12 \cdot 17 \cdot 16 \cdot 13,14)=\mathrm{I}_{12 \mathrm{p}} * \mathrm{X}_{12} * 0.35 * 0.001$

$\mathrm{F}(12 \cdot 17 \cdot 16 \cdot 13,14)=$ flow of TRU across the rumen wall for nonpulmonary deposits, activity/day

$\mathrm{I}_{120}=$ inhalation rate, $\mathrm{m}^{\mathrm{s}} / \mathrm{day}$, for $\mathrm{kth}$ population category, lth animal category

$\mathrm{X}_{12}=$ activity $/ \mathrm{m}^{\mathrm{s}}$ including fire increment to total air acivity if simulated 
58. $\mathrm{F}(5 \cdot 7 \cdot 17 \cdot 16 \cdot 13,14)=0.001 * \mathrm{~F}(10,13 \cdot 16 \cdot 17)$

$\mathrm{F}(5 \cdot 7 \cdot 17 \cdot 16 \cdot 13,14)=$ flow of TRU across rumen from consumed forage, activity/day $\mathrm{F}(10,13 \cdot 16 \cdot 17)=$ flow of TRU from soil into the rumen, activity/day

59. $F(13 \cdot 16 \cdot 17,9)=F(10 \cdot 13 \cdot 16 \cdot 17,14)+F(18 \cdot 17 \cdot 16 \cdot 13,14)+F(12 \cdot 17 \cdot 16 \cdot 13,14)$

$$
+\mathrm{F}(5 \cdot 7 \cdot 17 \cdot 16 \cdot 13,14) /\left(0.999 * \mathrm{I}_{1221} * \mathrm{C}_{84}\right)
$$

$\mathrm{F}(13 \cdot 16 \cdot 17,9)=\mathrm{T}$ 'RU flow entering rumen from all sources, activity/day

$F(10 \cdot 13 \cdot 16 \cdot 17,14)=$ flow of TRU from soil sources across rumen wall, activity/day

$\mathrm{F}(18 \cdot 17 \cdot 16 \cdot 13,14)=$ flow of TRU from pulmonary region of lungs to the gut and across the rumen wall, activity/day

$\mathrm{F}(12 \cdot 17 \cdot 16 \cdot 13,14)=$ flow of TRU across the rumen wall from nonpulmonary deposits

$\mathrm{F}(5 \cdot 7 \cdot 17 \cdot 16 \cdot 13,14)=$ flow of TRU across rumen from consumed forage, activity/day

$\mathrm{I}_{122}=$ fraction of region grazed by specific animal unit

$C_{94}=$ area of regional grid $(M 7)=3.73 \times 10^{8} \mathrm{~m}^{2}$

60. $\mathrm{P}(14,15)=\mathrm{X}_{14}{ }^{*} \mathrm{C}_{\mathrm{s2k}} * 0.001 /\left(\mathrm{I}_{180 \mathrm{k}} * 0.9\right)$

$$
=0
$$

$\mathrm{F}(14,15)=$ flow of TRU to milk, activity/day

$\mathrm{X}_{14}=$ biomass, $\mathrm{kg}$, for animal classes specified

$\mathrm{C}_{32}=$ fraction of population by age $(Z)$, see App. $C$

$\mathrm{l}_{130}=$ blood weight $(\mathrm{kg})$ for $\mathrm{kth}$ population category, lth animal category

61. $F(15, P)=1.0=$ flow of TRU from milk out of system, activity/day

62. $\mathrm{F}(4 \cdot 5,20 \mathrm{k})=\mathrm{I}_{1621-1}+\mathrm{I}_{1281} *\left(\mathrm{I}_{120 \mathrm{k}-\mathrm{l}}-\mathrm{I}_{188 \mathrm{k}}\right) / \mathrm{I}_{188 \mathrm{k}}+\mathrm{I}_{188 \mathrm{k}-1}$

$\mathrm{I}_{1341}>0.0$

$=\mathrm{I}_{1421}$

$\mathrm{I}_{1341}>0.0$

$=I_{184 !} *\left(X_{20 k} / I_{1881}\right) *\left[\left(\sum_{k=2}^{n} I_{t 38 k}\right)-I_{188 k}\right] / \sum_{k=2}^{n} I_{188 k}$

$I_{188 k} \leq 0.0$

$I_{1 s c k} \leq 0.0$

$\mathrm{F}(4 \cdot 5,20 \mathrm{i})=$ intraflow of biomass $\mathrm{is}$ a transport to diameter class $(\mathrm{k})$ from net photosynthesis $\mathrm{I}_{142}=$ growth allocation factor, $\mathrm{ft}^{\mathrm{s}}$

$I_{188}=$ total advantage factor for ith diameter class for biomass allocation

$\mathbf{I}_{\mathbf{1 3 4}}=$ growth increment, $\mathrm{ft}^{\mathbf{3}} / \mathrm{yr}$, for specific plant in a given year

$\mathrm{X}_{20}=$ volume, $\mathrm{ft}^{2}$, of specific plant with diameter $(\mathrm{k})$

$I_{s s}=$ virtual volume, $f^{n}$, required for setting radial growth rate of boles 
63. $\mathrm{F}(20 \mathrm{k}, 20 \mathrm{k}+1)=\mathrm{I}_{142 \mathrm{k}} *\left(\mathrm{I}_{2 \mathrm{sgk-1}-1}-\mathrm{I}_{120 \mathrm{k}}\right) /\left(\mathrm{I}_{198 \mathrm{k}}+\mathrm{I}_{28 \mathrm{~kg}-1}\right), \quad 2 \leq \mathrm{k} \leq 60$

$F(20 k, 20 k+1)=$ biomass transport from class $(k)$ to class $(k+1)$

$\mathrm{I}_{142}=$ growth allocation factor, $\mathrm{ft}^{\mathrm{s}}$

$I_{198}=$ total advantage factor for kth diameter class for biomass allocation

64. $\left.F(20 k, P)=X_{20 k} * I_{47} /\left(C_{271} *\left(\sum_{k=1}^{n} I_{188 k}\right)-I_{198 k}\right) / \sum_{k=1}^{n} I_{188 k}\right) \quad 2 \leq n \leq 60$

$\mathrm{F}(20 \mathrm{k}, \mathrm{P})=$ mortality flow of wood from a diameter class $(\mathrm{k}), \mathrm{ft}^{3} /$ day

$\mathrm{X}_{20}=$ volume, $\mathrm{ft}^{3}$, of specific plant with diameter $(\mathrm{k})$

$I_{47}=$ fractional crown cover for specific plani

$\mathrm{C}_{27}=$ mean average life-span (HF) of plant, years, see App. $\mathrm{C}$

$\mathrm{I}_{18 \mathrm{~s}}=$ total advantage factor for kth diameter class for biomass allocation

65. $20 \mathrm{k}=20 \mathrm{k}$

$$
\begin{aligned}
& I_{197 \mathrm{k}}>I_{144 \mathrm{k}} * 77.3 / \mathrm{I}_{19 \mathrm{si}} \\
& I_{187 \mathrm{k}} \leq I_{144 \mathrm{k}} * 77.3 / \mathrm{I}_{28 \mathrm{~s}}
\end{aligned}
$$$$
=0
$$

$\mathrm{I}_{137}=$ volume, $\mathrm{ft}^{\mathrm{s}}$, of specific plant with diameter $(\mathrm{k})$

$I_{144}=$ volume of tree with diameter $(k)$

$I_{135}=$ virtual volume, $\mathrm{ft}^{\mathrm{s}}$, required for setting growth rate of boles

66. $\mathrm{X}_{10 \mathrm{k}}=\mathrm{X}_{20 \mathrm{k}} / \mathrm{I}_{144 \mathrm{k}}$

$I_{144}=$ volume of tree with diameter $(k)$

67. $\mathrm{F}(\mathrm{P}, 20 \mathrm{k})=\mathrm{I}_{1481} * \mathrm{I}_{18 \mathrm{gk}} \int_{\mathrm{k}=2}^{\mathrm{n}} \mathrm{I}_{188 \mathrm{k}}$

$$
=I_{1431} *\left(1 / I_{188 k}\right) / \sum_{k=2}^{n} 1 / I_{188 k}
$$

$F(P, 20 k)=$ adjusted biomass flow into each diameter class $(k>1), f^{3 / y e a r}$

$\mathrm{I}_{18 \mathrm{a}}=$ total advantage factor for ith diameter class for biomass allocation

$I_{149}=$ growth allocation factor, $\mathrm{ft}^{\mathrm{s}}$

68. $F(5 \cdot 4,20 \mathrm{k})=\left|I_{1341}\right| * I_{1461} *\left(I_{1881} / I_{1861}\right)$

$\mathrm{F}(5 \cdot 4,20 \mathrm{k})=$ addition of TRU during current year

$I_{134}=$ growth increment, $\mathrm{ft}^{\mathrm{s}} /$ year, for specific plant in a given year .

$\mathbf{l}_{135}=$ virtual stand volume, $\mathrm{ft}^{\mathrm{a}}$, required for setting growth rate of boles

$\mathbf{l}_{138}=$ wood of stand, $\mathrm{ft}^{3}$

$I_{146}=$ change in specific activity, activity $/ \mathrm{m}^{2}$, from one year to the next 
69. $\left.\mathrm{F}(\mathrm{P}, 20 \mathrm{k})=\left(\mathrm{I}_{2 v ! \prime} / 0.142\right) *\left(\mathrm{X}_{20 \mathrm{k}}\right)_{\mathrm{p}} / \mathrm{X}_{\mathbf{2 0 k}} / 0.142\right)$

$\mathrm{F}(\mathrm{P}, 20 \mathrm{k})=$ mortality losses of TRU from class diameter $(\mathrm{k})$

$I_{147}=$ change in volume for succeeding years

$\mathrm{X}_{\text {sok }}=$ volume, $\mathrm{ft}^{\mathrm{a}}$, of specific plant with diameter $(\mathrm{k})$

$p=$ TRU material

70. $\mathrm{F}(20 \mathrm{k}, \mathrm{P})=\left(\mathrm{I}_{1471} / 0.142\right) *\left(\mathrm{X}_{20 \mathrm{k}}\right)_{\mathrm{p}} /\left(\mathrm{X}_{\mathbf{2 0 k}} / 0.142\right)$

$\mathrm{F}(20 \mathrm{k}, \mathrm{P})=$ mortality losses of TRU from class diameter $(\mathrm{k})$

$I_{147}=$ change in volume for succeeding years

$\mathrm{X}_{\text {sol }}=$ volume, $\mathrm{ft}^{\mathrm{2}}$, of specific with diameter $(\mathrm{k})$

$\mathrm{p}=$ TRU material 


\section{APPENDIX B}

\section{EQUATIONS INVOLVING SECONDARY INTERMEDIATE VARIABLES}

\section{EQUATIONS INVOLVING INTERMEDIATE VARIABLES}

1. $I_{s}=C_{\downarrow} /\left(1-\operatorname{EXP}\left(-C_{8} * I_{10}\right)\right)$

$I_{s}=$ soil evapotranspiration adjustment for soil depth

$\mathrm{C}_{4}=$ soil depth adjustment, 0.632

$\mathrm{C}_{\mathrm{b}}=$ soil depth exponential adjustment, 0.004

$\mathrm{I}_{19}=$ soil depth, $\mathrm{mm}$

2. $\mathrm{I}_{1 \mathrm{~s}}=\mathrm{C}_{2}+\mathrm{C}_{8}^{*} \mathrm{D}_{2}$

$I_{1 \mathrm{~s}}=$ potential evapotranspiration rate, $\mathrm{mm}$, from top $250 \mathrm{~mm}$ soil/day

$\mathrm{C}_{2}=$ evapotranspiration intercept, $0.6 \mathrm{~mm} /$ day

$\mathrm{C}_{\mathrm{a}}=$ evapotranspiration proportinality, $0.18 \mathrm{~mm} / \mathrm{day} /{ }^{\circ} \mathrm{C}$

$\mathrm{D}_{2}=$ daily temperature, ${ }^{\circ} \mathrm{C}$

3. $\mathrm{I}_{21}=1-\left(\mathrm{D}_{2}-\mathrm{C}_{6}\right)^{2} / \mathrm{C}_{7}$

$\mathrm{D}_{\mathbf{2}}>\mathrm{C}_{\mathbf{a}}$

$$
=1-\left(D_{2}-C_{0}\right)^{2} / C_{8}
$$

$\mathrm{D}_{2} \leq \mathrm{C}_{0}$

$\mathrm{I}_{21}=$ effect of temperature $\left({ }^{\circ} \mathrm{C}\right)$ on photosynthesis

$\mathrm{D}_{2}=$ daily temperature, ${ }^{\circ} \mathrm{C}$

$\mathrm{C}_{0}=$ optimum growth temperature, ${ }^{\circ} \mathrm{C}$, see App. $\mathrm{C}$

$\mathrm{C}_{7}=$ effect of temperature, above optimum, ${ }^{\circ} \mathrm{C}$, see App. $\mathrm{C}$

$\mathrm{C}_{8}=$ effect of temperature, below optimum, ${ }^{\circ} \mathrm{C}$, see App. $\mathrm{C}$

4. $\left.\mathrm{I}_{22}=1-\operatorname{EXP}\left(-\left(\mathrm{C}_{9} / \mathrm{I}_{19}-\mathrm{I}_{17}\right)\right) *\left(\mathrm{X}_{1}-\mathrm{I}_{27}\right)\right)+0.01$

$I_{22}=$ effect of soil moisture on photosynthesis for plant(l)

$\mathrm{C}_{0}=$ effect of soil moisture on photosynthesis, see App. C

$\mathrm{I}_{10}=$ field capacity of soil, $\% \mathrm{H}_{2} \mathrm{O}$

$I_{17}=$ wilting point of soil, $\% \mathrm{H}_{2} \mathrm{O}$

$\mathrm{X}_{1}=$ current value of soil moisture availability

5. $\mathrm{I}_{23}=\mathrm{C}_{10} * \mathrm{D}_{\mathrm{s}}$

$I_{2 \mathrm{~s}}=$ maximum photosynthetic efficiency (grams $\mathrm{CH}_{3} \mathrm{O} / \mathrm{m}^{2} /$ day)

$\mathrm{C}_{10}=$ effect of solar radiation on photosynthesis, ${ }^{2} 0.001 / \mathrm{Ly} /$ day

$\mathrm{D}_{\mathbf{3}}=$ solar radiation $\mathrm{Ly} / \mathrm{day}$ 
6. $I_{27}=\left(X_{4}+X_{8}\right)$

$\mathrm{I}_{27}=\mathrm{g} / \mathrm{m}^{2}$ live above ground biomass

$\mathrm{X}_{4}=\mathrm{g} / \mathrm{m}^{2}$ live above ground biomass

$\mathrm{X}_{0}=$ needle or leaf biomass, $\mathrm{g} / \mathrm{m}^{2}$

7. $\mathrm{I}_{28}=\sum_{\mathrm{i}=1}^{\mathrm{I}_{80}} \mathrm{I}_{291} / \mathrm{I}_{\mathbf{3 0}}$

where

$$
\begin{aligned}
\mathrm{I}_{201} & =\mathrm{I}_{291} & \mathrm{~L}_{\mathrm{b}}=1 \\
& =\mathrm{X}_{5} & \mathrm{~L}_{b}=0 ; \mathrm{X}_{\mathrm{b}}<\mathrm{I}_{201}
\end{aligned}
$$

$L_{6}=$ distinguishes woody plants $(0)$ from herb types(1)

$\mathrm{X}_{6}=$ needle or leaf biomass

$\mathrm{I}_{2 \mathrm{a}}=$ running average productivity $\mathrm{kg} / \mathrm{m}^{2} / \mathrm{yr}$

$\mathrm{I}_{20}=$ net yearly productivity $\mathrm{kg} / \mathrm{m}^{2} / \mathrm{yr}$

$\mathrm{I}_{s_{0}}=$ current year number

8.

$$
I_{2 \mathrm{~g}}=\sum_{i=1}^{365} I_{351}
$$

$\mathrm{I}_{29}=$ net yearly productivity, $\mathrm{g} / \mathrm{m}^{2} / \mathrm{yr}$

$I_{s k}=\mathrm{g} / \mathrm{m}^{2}$, biomass net production on a given day

9. $I_{83}=\left(C_{16}-C_{17}\right) /\left(I_{21} * I_{28}\right)$

$I_{b 0}=$ maximum yearly water utilization efficiency for photosynthesis, $\mathrm{m} \ell / \mathrm{g} \mathrm{CH}_{2} \mathrm{O}$

$\mathrm{I}_{21}=$ effect of temperature $\left({ }^{\circ} \mathrm{C}\right)$ on photosynthesis for plant

$I_{22}=$ effect of soil moisture on photosynthesis for piant

$\mathrm{C}_{16}=$ minimum water utilization efficiency, $\mathrm{m} \ell / \mathrm{g}$, see App. $\mathrm{C}$

$\mathrm{C}_{17}=$ Hatch-Slack coefficient (PSE), see App. C

10. $I_{34}=F(P, 5)-F(3, P)-F(4, P)-F(5, P)$

$\mathrm{I}_{34}=$ net daily biomass production, $\mathrm{g} / \mathrm{m}^{2} /$ day

$\mathrm{F}(\mathrm{P}, 5)=$ potential gross photosynthesis, see App. A

$\mathrm{F}(3, \mathrm{P})=$ root respiration, $\mathrm{g} / \mathrm{m}^{2}$, see A.pp. A

$F(4, P)=$ respiration of stems, $g / \mathrm{m}^{2}$, see App. A

$\mathrm{F}(5, \mathrm{P})=$ respiration of leaves, $\mathrm{g} / \mathrm{m}^{2}$, see App. A

11. $I_{98}=I_{34} * I_{s 6} * I_{57}$

$$
\left.=I_{34} * I_{30}{ }^{2} * I_{37}{ }^{2} * I_{20} * 103 / I_{38} * I_{s 4}\right)
$$

$$
\begin{aligned}
& \mathrm{I}_{38} * \mathrm{I}_{34} \leq \mathrm{I}_{20} * 10^{2} * \mathrm{I}_{38} * \mathrm{I}_{37} \\
& \mathrm{I}_{33} * \mathrm{I}_{34}>\mathrm{I}_{20} * 10^{*} * \mathrm{I}_{30} * \mathrm{I}_{37}
\end{aligned}
$$


$\mathrm{I}_{2 \mathrm{a}}=$ net biomass production on given day, $\mathrm{g} / \mathrm{m}^{2}$

$\mathrm{I}_{20}=$ adjusted evapotranspiration rate. $\mathrm{mm} / 250 \mathrm{~mm}$

$I_{s s}=$ maximum yearly water utilization efficiency for photosynthesis, $\mathrm{m} \ell / \mathrm{g} \mathrm{CH}_{2} \mathrm{O}$

$I_{34}=$ net daily biomass production, $\mathrm{g} / \mathrm{m}^{2} /$ day

$\mathrm{I}_{30}=$ daily niche competition factor, $\mathrm{g} / \mathrm{m}^{2}$

$I_{37}=$ succession factor

12. $\mathrm{I}_{38}=\mathrm{I}_{88} * \mathrm{I}_{80} * \mathrm{I}_{87}$

$\mathrm{I}_{3 \mathrm{~B}}=\mathrm{g} / \mathrm{m}^{2}$, biomass net production on a given day

$l_{3 \mathrm{~B}}=$ daily niche competition factor, $\mathrm{g} / \mathrm{m}^{2}$

$l_{37}=$ succession factor

13. $I_{381}=I_{931} * I_{981} * I_{371}$

$\mathrm{I}_{981}=\mathrm{g} / \mathrm{m}^{2}$, biomass net production on a given day

$I_{381}=$ daily structural biomass competition factor, $\mathrm{g} / \mathrm{m}^{2}$

$l_{971}=$ succession factor

14. $\mathrm{I}_{3 \theta}=\mathrm{I}_{3 \theta}$

$\mathrm{O}_{2}=1.0$

$=1.0$

$\mathrm{O}_{2}=0.0$

$\mathrm{I}_{s e}=$ daily structural biomass competition factor, $\mathrm{g} / \mathrm{m}^{2}$

$\mathrm{O}_{2}=$ determines whether succession module is used(1) or ignored(0)

15.

$$
\begin{array}{rlr}
I_{981} & \left.=\left[\left(I_{s s 1} / C_{271} * X_{s}\right)\right] / \sum_{k=1}^{n} I_{s s k} /\left(C_{27 k} * X_{8 k}\right)\right] & I_{9 s}>0 \\
& =1.0 & I_{3 s} \leq 0
\end{array}
$$

$\mathbf{l}_{\mathrm{se}}=$ daily structural biomass competition factor, $\mathrm{g} / \mathrm{m}^{2}$

$I_{35}=\mathrm{g} / \mathrm{m}^{2}$, biomass net production on a given day

$\mathrm{C}_{\mathrm{L}^{7}}=$ mean average life-span $(\mathrm{HF})$, years, see App. C

$\mathrm{X}_{\mathrm{o}}=$ needle or leaf biomass $\mathrm{kg} / \mathrm{m}^{2}$

16. $\mathrm{I}_{37}=\mathrm{I}_{97}$

$\mathrm{O}_{1}=1.0$

$$
=1.0
$$

$\mathrm{O}_{1}=0.0$

$\mathrm{I}_{37}=$ succession factor calculated on yearly basis

$\mathrm{O}_{1}=$ determines which plant groups are to be considered for competition of available land area

17.

$$
I_{s 7}=I_{881} / \sum_{k=1}^{n} I_{80 k}
$$


$I_{27}=$ succession factor

$I_{\omega}=$ succession factor

18. $I_{20}=2 /\left(365 * C_{27}\right)$

$I_{88}=$ factor to determine mortality of certain plant types

$\mathrm{C}_{27}=$ mean average life-span ( $\mathrm{HF}$ ), years, see App. C

19. $I_{60}=0.004+0.0032 * D_{2}$

$I_{10}=$ dead biomass temperature decay factor

$\mathrm{D}_{2}=$ daily temperature, ${ }^{\circ} \mathrm{C}$

20. $\mathrm{I}_{41}=1.0+\left(1 /\left(\mathrm{I}_{10}-\mathrm{I}_{17}\right) *\left(\mathrm{X}_{1}-\mathrm{I}_{27}\right)\right.$

$\mathrm{I}_{41}=$ dead biomass soil moisture decay factor

$I_{10}=$ field capacity of soil, $\% \mathrm{H}_{2} \mathrm{O}$

$\mathrm{I}_{17}=$ wilting point of soil, $\% \mathrm{H}_{2} \mathrm{O}$

$\mathrm{X}_{1}=$ current value of soil moisture availability

21. $\mathrm{I}_{62}=2.5 * \operatorname{EXP}\left(-4.6 * \mathrm{I}_{17}\right) \times 10^{-8}$

$I_{62}=$ resuspension factor, day $^{-1}$

$I_{47}=$ fractional crown cover for specific plant

22. $I_{47}=\left(1-\operatorname{EXP}\left(-1.54 * I_{28}\right) * I_{141}\right)$

$\mathrm{I}_{47}=$ fractional crown cover for specific plant

$\mathrm{I}_{2 \mathrm{~g}}=$ running average productivity, $\mathrm{kg} / \mathrm{m}^{2} / \mathrm{yr}$

$I_{141}=$ bole to crown conversion factor

23. $\mathrm{I}_{49}=0.013 * \mathrm{I}_{B 0}$

$\mathrm{I}_{40}=$ leaf area index $\mathrm{m}^{2} / \mathrm{m}^{2}$ for specific plant category

$I_{60}=$ edible portion of plants, $\mathrm{g} / \mathrm{m}^{2}$

24. $\mathrm{I}_{B 0}=\mathrm{X}_{6}+\mathrm{X}_{7}$

a) plants $(1,2,3,7,8,11,12,13,18,19)^{1}$

$$
=\mathrm{X}_{\mathrm{b}}
$$

b) plants $(4,5,6,9,10,14,15,16,17,20,21)$

$I_{50}=$ edible portion of plants, $\mathrm{g} / \mathrm{m}^{2}$

$\mathrm{X}_{\mathrm{b}}=$ needle or leaf biomass $\mathrm{kg} / \mathrm{m}^{2}$

$\mathrm{X}_{7}=\mathrm{g} / \mathrm{m}^{2}$ of dead standing biomass

25. $\mathrm{I}_{s \mathrm{~b}}=\left(1-\operatorname{EXP}\left(-0.001 *\left(\mathrm{I}_{40}-1\right) * 365+\mathrm{I}_{8}\right)\right) * 9144$

$$
=\left(1-\operatorname{EXP}\left(-0.001 * I_{B}\right)\right) * 9144
$$

$$
\mathrm{C}_{87} \leq 9.0
$$


$I_{B S}=$ root penetration, $\mathrm{mm}$, as a function of total days elapsed

$\mathrm{I}_{\mathrm{s}}=$ day number

$I_{16}=$ year number

$\mathrm{C}_{\tilde{z} 7}=$ mean average life-span $(\mathrm{HF})$, years, see App. $\mathrm{C}$

26.

$I_{601}=\sum_{i=1}^{I_{46}}\left[\left(I_{271}+X_{81}+X_{01}+X_{71}+X_{81}+X_{01}\right) / C_{271}\right] / \sum_{k=1}^{I_{48}} k$

$\mathrm{I}_{\mathrm{be}}=$ structural biomass succession fac tor, $\mathrm{g} / \mathrm{m}^{2} / \mathrm{yr}$

$\mathrm{I}_{27}=\mathrm{g} / \mathrm{m}^{2}$ live above ground biomass

$\mathrm{X}_{\mathbf{3}}=\mathrm{g} / \mathrm{m}^{2}$ live root biornass

$\mathrm{C}_{27}=$ mean average life-span $(\mathrm{HF})$, years, see App. $\mathrm{C}$

$\mathrm{I}_{\mathbf{4 0}}=$ year number

27. $\mathrm{I}_{67}=[\operatorname{EXP}(-0.004 * 25 \mathrm{C} *(\mathrm{i}-1))-\operatorname{EXP}(-0.004 * 250 \mathrm{i})] / \operatorname{EXP}\left(-0.004 * \mathrm{I}_{84}\right)$

where

$\left(2 \leq \mathrm{i} \leq \mathrm{I}_{34}\right)$

$I_{67}=$ root fraction in given soil horizon

$I_{s 4}=$ number of soil horizons in $250 \mathrm{~mm}$ intervals

28. $I_{88}=\left[\left[I_{80} /\left(X_{0}+C_{11} * X_{8}\right)\right] *\left[1-I_{80} d\left(X_{6}+C_{11} * X_{8}\right)\right]\right]_{b}$

$I_{s 8}=$ biomass transpiration coefficient, day $^{-1}$

$\mathrm{X}_{\mathrm{s}}=$ needle or leaf biomass, $\mathrm{g} / \mathrm{m}^{2}$

$\mathrm{I}_{00}=$ net daily biomass production shunted to roots, $\mathrm{g} / \mathrm{m}^{2} /$ day

$\mathrm{C}_{11}=$ respiratory maintenance component (ARP), see App. C

$\mathrm{I}_{27}=$ live above ground biomass, $\mathrm{g} / \mathrm{m}^{2}$

$\mathbf{b}=$ biomass flow parameters

29. $I_{80}=|F(4 ; 3)|$

$$
=\left(\mathrm{X}_{8}+\mathrm{C}_{11} * \mathrm{X}_{6}\right) \mathrm{b}
$$

$\mathrm{F}(4,3) \leq\left(\mathrm{X}_{\mathrm{b}}+\mathrm{C}_{11} * \mathrm{X}_{\mathrm{b}}\right)_{b}-1.0$

$\mathrm{F}(4,3)>\left(\mathrm{X}_{\mathrm{s}}+\mathrm{C}_{11} * \mathrm{X}_{\mathrm{s}}\right)_{\mathrm{b}}$

$\mathrm{I}_{80}=$ net deily biomass production shunted to roots $\mathrm{g} / \mathrm{m}^{2} /$ day

$\mathrm{X}_{\mathrm{0}}=$ needle or leaf biomass

$\mathrm{C}_{11}=$ respiratory maintenance component (AR), see App. C

$\mathrm{F}(4,3)=$ transport of photosynthate to roots from above ground parts, $\mathrm{g} / \mathrm{m}^{2}$, see Fig. 7 Flows, No. 13 
30. $I_{801}=I_{741} * I_{791}+\left(1-I_{741}\right) * C_{81}$

$$
=\mathrm{C}_{81}
$$

$I_{00}=$ nitrogen content, percentage, of consumed biomass

$I_{74}=$ fraction of diet composed of green biomass for ith plant

$I_{79}=$ initial leaf biomass

$\mathrm{I}_{7 \mathrm{~b}}=$ nitrogen content, percentage, of standing green biomass

$\mathrm{C}_{\mathrm{s} 1}=$ nitrogen content of dry forage (N3), $0.6 \%$

31. Plant

Rank

$\mathrm{I}_{871}=\mathrm{I}_{671}$

$I_{67 j}=I_{47 j} *\left(1-I_{071}\right)$

$I_{071}=I_{671} *\left(1-I_{671}-I_{87}\right)$

$\dot{I}_{s 7 m}=I_{47 m} *\left(1-I_{\theta 71}-I_{67 j} \ldots I_{67 m}\right)$

$\mathrm{I}_{\mathrm{ar}}=$ succession factor

$I_{47}=$ fractional crown cover for specific plant

32. $\mathrm{I}_{681}=\left(\mathrm{I}_{671} / \mathrm{I}_{671}\right) *\left(\mathrm{I}_{601} / /_{60}\right)$

$\mathrm{I}_{\mathrm{ag}}=$ succession factor

$\mathrm{I}_{\mathrm{a} 7}=$ succession ranking factor

$I_{67}=$ fractional crown cover for specific plant

$I_{\delta 8}=$ structural biomass succession factor, $\mathrm{g} / \mathrm{m}^{2} / \mathrm{yr}$ (yearly running average)

$\mathrm{I}_{69}=$ maximum running average structural biomass succession factor, $\mathrm{gm} / \mathrm{m}^{2} / \mathrm{yr}$

33. $I_{711}=0.7, i=1, \quad I_{70 \jmath, \mathrm{k}}=1$

$$
\begin{array}{ll}
=0.2, \mathrm{i}=2, & \mathrm{I}_{70 \mathrm{j}, \mathrm{k}}=2 \\
=0.1, \mathrm{i}=3, & \mathrm{I}_{70 \mathrm{~J}, \mathrm{k}}=3
\end{array}
$$

$\mathrm{I}_{71}=$ unimal preference fraction for ith food preference category

$I_{70}=$ plants utilized by kth animal category

34. $I_{71_{1}}=0.78, \quad I_{70 k, k}=1$

$I_{71_{2}}=0.22, \quad I_{70 \mathrm{~J}, \mathrm{k}}=2$

$$
\mathrm{I}_{7 \mathrm{~s}_{\mathrm{g}}}=0.0
$$

$I_{71}=$ animal preference fraction for ith preference category

$I_{7}=$ total sum of plants utilized for ith food preference category

$\mathrm{I}_{70}=$ plants utilized by kth animal category 
35. $\quad I_{71_{1}}=1, \quad I_{70 S_{, k}}=1$

$\mathrm{I}_{70}=$ plants utilized by kth animal category

$$
\mathrm{I}_{7 \mathrm{~s}_{\mathrm{g}}}=0.0
$$

$I_{71}=$ animal preference fraction for kth preference category

$I_{78}=$ total sum of plants utilized for ith food preference category

36.

$$
\mathrm{I}_{721}=\sum_{\mathrm{j}=1}^{\mathrm{I}_{731}}\left[\left(\mathrm{I}_{7 \mathrm{j}}\right) * \mathrm{X}_{\mathrm{bj}}+\left(1-\mathrm{I}_{74 \mathrm{j}}\right) * \mathrm{X}_{7 \mathrm{j}} * \mathrm{X}_{\mathrm{b} J} / \mathrm{I}_{27 \mathrm{~J}}\right] * \mathrm{I}_{78 \mathrm{~s}}
$$

$I_{72}=$ available forage $\mathrm{g} / \mathrm{m}^{2}$ from all forage sources of ith food preference category

$I_{73}=$ total sum of plants utilized in ith food preference category

$I_{74}=$ fraction of diet composed of green biomass for plant

$I_{7 s}=$ utilization efficiency, fraction of standing forage

$I_{27}=\mathrm{g} / \mathrm{m}^{2}$ live above ground biomass

$\mathrm{X}_{8}=$ needile or leaf biomass

$\mathrm{X}_{7}=\mathrm{g} / \mathrm{m}^{2}$ of dead standing biomass

37. $\mathrm{I}_{74 \mathrm{j}}=3 * \mathrm{X}_{\mathrm{s}} /\left(3 * \mathrm{X}_{8 \mathrm{~J}}+\mathrm{X}_{7 \mathrm{j}} * \mathrm{X}_{\mathrm{s}} / \mathrm{I}_{\mathbf{2 7}}\right)$

$I_{74}=$ fraction of diet composed of green biomass for jth plant

$\mathrm{X}_{5}=$ needle or leaf biomass, $\mathrm{kg} / \mathrm{m}^{2}$

$\mathrm{X}_{7}=\mathrm{g} / \mathrm{m}^{2}$ of dead standing biomass

$\mathrm{I}_{27}=\mathrm{g} / \mathrm{m}^{2}$ live above ground biomass

38. $\mathrm{I}_{76 \mathrm{j}}=\left(1-\operatorname{EXP}\left(-0.167\left(\mathrm{X}_{8 \mathrm{j}}+\mathrm{X}_{7 j} * \mathrm{X}_{6} / \mathrm{I}_{27 \mathrm{j}}\right)\right)\right)$

$I_{78}=$ utilization efficiency, fraction of standing forage for $j$ th plant

$\mathrm{X}_{\mathrm{s}}=$ needle or leaf biomass, $\mathrm{kg} / \mathrm{m}^{2}$

$\mathrm{X}_{7}=\mathrm{g} / \mathrm{m}^{2}$ of dead standing biomass

$\mathrm{I}_{27}=\mathrm{g} / \mathrm{m}^{2}$ live above ground biomass

39. $\mathrm{I}_{761}=\mathrm{C}_{19} * \mathrm{I}_{98} / \mathrm{X}_{\mathrm{b}}$

$$
=0.0
$$

$\mathrm{I}_{76}=$ growth rate, fraction/day, for ith plant

$\mathrm{C}_{10}=$ fraction of photosynthate remaining above ground (STRANS), App. C

$\mathrm{I}_{\mathrm{sa}}=\mathrm{g} / \mathrm{m}^{2}$ biomass net production on a given day

$\mathrm{X}_{\mathrm{s}}=$ needle or leaf biomass, $\mathrm{g} / \mathrm{m}^{2}$

40. $I_{771}=3.0$

$$
=(10.9) * I_{761}+1.436
$$

$I_{77}=$ nitrogen content, percentage, of photosynthate for ith plant

$I_{76}=$ growth rate, fraction/day, for plant category 
41. $I_{701}=I_{701} *\left(I_{701} / X_{61}\right)+\left(1-I_{701} / X_{81}\right) * I_{771}$

$I_{16}=$ nitrogen content, percentage, of standing green biomass for ith plant

$I_{n}=$ initial leaf biomass

$I_{n}=$ nitrogen content, percentage, of photosynthate

$\mathrm{X}_{\mathrm{a}}=$ needle or leaf biomass, $\mathrm{kg} / \mathrm{m}^{2}$

42. $\mathrm{I}_{701}=\mathrm{I}_{701}$

$$
=\left(X_{01}\right)^{2 / I_{791}}
$$$$
I_{701} \leq X_{81}
$$

$I_{n}=$ initial leaf biomass for ith plant

$\mathrm{X}_{\mathbf{1}}=$ needle or leaf biomass, $\mathrm{kg} / \mathrm{m}^{2}$

43. $l_{201}=I_{711} * I_{811}$

$I_{00}=$ uptake, $\mathrm{kg} /$ day, for ith preference category

$I_{01}=$ ingestion rate, $\mathrm{kg} /$ day

$I_{n 1}=$ animal preference fraction for ith preference category

44.

$$
I_{82 k}=\sum_{i=1}^{n}\left(I_{80} / I_{n 1 k}\right) * \sum_{j=1}^{I_{73}}\left(I_{1811}\right) / I_{n a}
$$

$I_{12}=$ nitrogen content of ingested forage, \%

$I_{s 0}=$ uptake, $\mathrm{kg} /$ day, for ith preference category

$\mathrm{I}_{\mathrm{n}}=$ ingestion rate, $\mathrm{kg} /$ day

$I_{n 2}=$ total sum of plants utilized for $\mathrm{kth}$ animal category

$I_{161}=$ sum of total nitrogen concentration $\times 100$ for jth plant in preference group

$I_{73}=$ available forage, $\mathrm{g} / \mathrm{m}^{2}$, from all forage sources

45. $I_{821}=I_{821}$

$$
\begin{aligned}
& I_{321}>C_{31}, i>1 \\
& i=1 \\
& I_{821} \leq C_{31}, i>1
\end{aligned}
$$$$
=3.0
$$$$
=\mathrm{C}_{\mathbf{3 1}}
$$

$I_{n}=$ nitrogen content of ingested forage, $\%$ for ith animal age group

$\mathrm{C}_{\mathbf{2 1}}=$ nitrogen content of dry forage, $0.6 \%$

46.

$$
I_{8 s k}=\sum_{i=1}^{n}\left(I_{s 0 V} / I_{81 k}\right) \sum_{j=1}^{I_{7 a 1}}
$$

$I_{a}=$ cumulative green biomass uptake for kth food preference category

$\mathrm{I}_{\mathbf{}_{0}}=$ uptake, $\mathrm{kg} /$ day, for ith plant in preference category

$\mathrm{I}_{01}=$ ingestion rate, $\mathrm{kg} /$ day

$I_{n}=$ total sum of plants utilized for ith animal category

$I_{m}=$ available green standing biomass for ith plant in preference category 
47. $I_{\text {as }}=I_{76 s} * X_{5 j} * I_{72} / I_{71}$

$\mathrm{I}_{\boldsymbol{\mu}}=$ available green standing biomass for $\mathrm{jth}$ preference category

$I_{72}=$ available forage, $\mathrm{g} / \mathrm{m}^{2}$, from all forage sources

$I_{74}=$ fraction of diet composed of green biomass

$I_{70}=$ utilization efficiency, fraction of standing green forage

$\mathrm{X}_{6}=$ needle or leaf biomass, $\mathrm{kg} / \mathrm{m}^{2}$

48.

$I_{86 k}=\sum_{i=1}^{n}\left(I_{801} / I_{81 k}\right) * \sum_{j=1}^{I_{7 a}} I_{8 \theta j}$

$I_{a b}=$ specific activity, activity/gram, for given category consumed daily by kth animal category

$I_{20}=$ uptake, $\mathrm{kg} / \mathrm{day}$, for ith plant in preference category

$\mathrm{I}_{\mathbf{0}}=$ ingestion rate, $\mathrm{kg} /$ day

$I_{\mathbf{n}}=$ total sum of plants utilized for kth animal category

$I_{a}=$ specific activity of $j$ th plant in preference category, activity/gram

49. $I_{80 j}=I_{78 j} * I_{78 j} *\left(X_{b} *\left(X_{4 j}+X_{6 j}\right)_{p}+\left(1-I_{74 j}\right) * X_{7} * X_{6} *\left(X_{7}\right) p / I_{27}\right) / I_{72}$

$\mathrm{I}_{\boldsymbol{\omega}}=$ specific activity of $\mathrm{jth}$ preference category, activity/gram

$I_{7 b}=$ utilization efficiency, fraction of standing green forage

$I_{74}=$ fraction of diet composed of green biomass

$\mathrm{X}_{\mathrm{b}}=$ needle or leaf biomass, $\mathrm{kg} / \mathrm{m}^{2}$

$\mathrm{X}_{\mathrm{c}}=\mathrm{g} / \mathrm{m}^{2}$ live above ground biomass

$\mathrm{X}_{7}=\mathrm{g} / \mathrm{m}^{2}$ of dead standing biomass

$I_{27}=\mathrm{g} / \mathrm{m}^{2}$ live above ground biomass

$I_{72}=$ available forage $\mathrm{g} / \mathrm{m}^{2}$ from all forage sources

$\mathrm{p}=$ TRU pool

50.

$I_{a 7 k}=\sum_{i=1}^{n}\left(I_{831} / I_{81 k}\right) * \sum_{j=1}^{I_{82}^{2}} I_{88 s}$

$I_{\mathbf{m}}=$ specific activity, activity/gram, on surface of given food category consumed by kth animal category

$\mathrm{I}_{\mathbf{m}}=$ uptake, $\mathrm{kg} /$ day, for ith plant in preference category

$\mathrm{I}_{\mathrm{BI}}=$ ingestion rate, $\mathrm{kg} /$ day

$I_{n}=$ total sum of plants utilized

$I_{m}=$ specific activity, activity/gram, for jth plant in food preference category (surface contamination)

51. $I_{88 \jmath}=I_{78 j} * I_{74 j} *\left(\left(X_{8} * X_{11 \jmath}\right) p+\left(1-I_{78 j}\right) * X_{7} * X_{b} *\left(X_{11}\right) p / I_{27}\right) / I_{72}$

$I_{u}=$ specific activity, activity/gram, for $j$ th food preference category (surface contamination)

$\mathrm{I}_{v}=$ utilization efficiency, fraction of standing green forage

$I_{14}=$ fraction of diet composed of green biomass 
$\mathrm{X}_{\mathrm{B}}=$ needle or leaf biomass, $\mathrm{kg} / \mathrm{m}^{2}$

$\mathrm{X}_{11}=$ activity $/ \mathrm{m}^{2}$ on leaf surface

$X_{7}=\mathrm{g} / \mathrm{m}^{2}$ of dead standing biomass

$\mathrm{I}_{27}=\mathrm{g} / \mathrm{m}^{2}$ live above ground biomass

$l_{72}=$ available forage, $\mathrm{g} / \mathrm{m}^{2}$, from all forage sources

$\mathrm{p}=$ TRU pool

52.

$I_{89 m}=\sum_{i=1}^{I_{78}}\left(I_{27}-20+\left(\left(I_{27}-20\right) / I_{27}\right) * X_{7}\right) * I_{801} * 4047$

$I_{\text {aq }}=$ biomass, grams, available to mth animal category from all plants utilized as supplementary feed during current year

$I_{n a}=$ total sum of plants utilized

$I_{m}=\mathrm{g} / \mathrm{m}^{2}$ live above ground biomass

$X_{1}=\mathrm{g} / \mathrm{m}^{2}$ of dead standing biomass

$I_{20}=$ number of acres/plant of ith plant utilized

53.

$I_{91 m}=\sum_{i=1}^{I_{7 s}}\left(\left(X_{41}+X_{61}+X_{71}\right) p /\left(I_{271}+X_{7 l}\right)\right) *\left(I_{271}-20+\left(\left(I_{271}-20\right) / I_{271}\right) * X_{71} * X_{901} * 4047\right.$

$I_{01}=$ total activity available to $\mathrm{mth}$ animal category from irrigated forage

$I_{n}=$ total sum of plants utilized

$\mathrm{X}_{4}=\mathrm{g} / \mathrm{m}^{2}$ of live above ground biomass of ith plant utilized

$\mathrm{X}_{\mathrm{b}}=$ needle or leaf biomass, $\mathrm{kg} / \mathrm{m}^{2}$

$\mathrm{X}_{7}=\mathrm{g} / \mathrm{m}^{2}$ of dead standing biomass

$\mathrm{I}_{n}=\mathrm{g} / \mathrm{m}^{2}$ live above ground biomass

$I_{n}=$ number of acres/plant

54.

$I_{92 m}=\sum_{i=1}^{I_{78}}\left(X_{11} /\left(I_{271}+X_{71}\right)\right) *\left(I_{271}-20+\left(\left(I_{271}-20\right) / I_{271}\right) * X_{71} * I_{801} * 4047\right.$

$I_{n}=$ total surface activity available to $m$ th animal category from irrigated forage

$I_{23}=$ total sum of plants utilized

$\mathrm{X}_{11}=$ activity $/ \mathrm{m}^{2}$ on leaf surface of ith plant utilized

$I_{n}=g / m^{2}$ live above ground biomass

$\mathrm{X}_{1}=\mathrm{g} / \mathrm{m}^{2}$ of dead standing biomass

$I_{\text {w0 }}=$ number of acres/plant

55. $\quad I_{98 m}=\left(I_{91 m}^{*} I_{90 m}+I_{94 m}+I_{98 m}\right) /\left(I_{90 m}+I_{08 m}\right)$

$\mathbf{I}_{\mathbf{w}}=$ total activity available to $\mathrm{mth}$ animal category from irrigated forage

$\mathrm{I}_{01}=$ total activity available to animal category from irrigated forage

$\mathbf{I}_{\boldsymbol{n}}=$ biomass, grams, available to animal category from all plants utilized as supplementary feed during current year

$I_{n}=$ previous year total activity available to animal category from irrigated forage

$I_{m}=$ previous year biomass, grams, available to animal category as supplementary feed 
56. $\quad I_{97 m}=\left(I_{92 m} * I_{99 m}+I_{98 m}\right) /\left(I_{99 m}+I_{98 m}\right)$

$I_{97}=$ total surface activity available to $\mathrm{mth}$ animal category from irrigated forage

$I_{02}=$ total surface activity available to animal category from irrigated forage

$I_{s 0}=$ biomass, grams, available to animal category from all plants utilized as supplementary feed during current year

$\mathrm{I}_{9 a}=$ previous year total surface activity available to animal category from irrigated forage

$\mathrm{I}_{93}=$ previous year biomass, grams, available to animal category as supplementary feed

57. $\mathrm{I}_{101 \mathrm{j}}=\mathrm{C}_{321} * \mathrm{I}_{100 \mathrm{~s}}$

$I_{101}=$ product of population structure and number of animal units on range

$I_{100}=$ number of animal units on range for $j$ th grazing interval

$\mathrm{C}_{32}=$ fraction of population by ith age group $(\mathrm{Z})$, see App. C

58. $I_{1091}=\left(C_{391} * I_{1021}\right)^{0.76}$ initially, and $I_{1091}=\left(X_{141}\right)^{0.76}$ thereafter

$I_{109}=$ metabolic biomass $(\mathrm{kg})$ for ith population category

$\mathrm{C}_{\mathrm{sa}}=$ initial weight of beef cattle by age $(\mathrm{W}), \mathrm{kg}$, see App. C

$I_{102}=$ animal weights, mature weight ratio

$\mathrm{X}_{14}=$ biomass, $\mathrm{kg}$

59. $I_{1041}=0.16 * I_{1031}$

$I_{104}=$ rumen capacity, $\mathrm{kg}$, for a given ith age (yr) category of specific animal category

$\mathrm{I}_{108}=$ metabolic biomass, $\mathrm{kg}$

60. $\mathrm{I}_{100}=\mathrm{I}_{108} * \mathrm{I}_{\mathrm{\theta 1}} * 4300$

$I_{100}=$ energy in digestible fraction of intake, kcal

$\mathrm{I}_{106}=$ digestible fraction of ingested forage

$\mathrm{I}_{81}=$ ingestion rate, $\mathrm{kg} / \mathrm{day}$

61. $\mathrm{I}_{108}=\left(\mathrm{X}_{16 \mathrm{~V}} / \mathrm{I}_{108 \mathrm{1}}\right) * 0.04$

$I_{108}=$ fermentation digestion rate, fraction $/ \mathrm{kg}$

$\mathrm{X}_{10}=$ microprotein level, grams, in rumen for ith age

$\mathrm{I}_{10 \mathrm{~s}}=$ metabolic biomass, $\mathrm{kg}$

62. $\mathrm{I}_{109}=0.25+\mathrm{I}_{108}$

$\mathrm{I}_{100} \leq 0.95$

$=0.95$

$\mathrm{I}_{20 \mathrm{c}}>0.95$

$I_{100}=$ rumen exit rate, fraction/day

$\mathrm{I}_{108}=$ fermentation digestion rate, fraction $/ \mathrm{kg}$ 
63. $\mathrm{I}_{110,1}=\mathrm{X}_{1 \mathrm{1u}}+\mathrm{X}_{111}+0.001 * \mathrm{X}_{111}$

$\mathbb{I}_{110}=$ rumen fill, $\mathrm{kg}$, for ith population age group of lth animal category

$X_{13}=$ rumen capacity, $\mathrm{kg}$, the ith age (yr) category

$X_{10}=$ accumulated activity entering pulmonary region for kth population age group, lth animal

$X_{10}=$ microprotein level, grams, in rumen for kth age, Ith animal

64. $I_{111}=0.747-0.15 *(n-0.6)$

$=0.747$

$\mathrm{I}_{\mathrm{g},}<0.6$

$=0.591$

$\mathrm{I}_{62}>1.8$

$I_{111}=$ percent of fermented energy as fatty acids

$\mathrm{I}_{\mathrm{a2}}=$ nitrogen content of ingested forage, $\%$

65. $\mathrm{I}_{112}=\mathrm{I}_{100} * \mathrm{I}_{108} * \mathrm{I}_{111}$

$I_{11 a}=$ fatty acid energy, $\mathrm{kcal}$

$I_{108}=$ energy in digestible fraction of intake, kcal

$\mathrm{I}_{108}=$ fermentation digestion rate, fraction $/ \mathrm{kg}$

$I_{11}=$ percent of fermented energy as fatty acids

66. $I_{113}=[0.25 *[F(13,9)+F(17,9)]] * 4300$

$\mathrm{I}_{128}=$ energy from passed food, kcal

$F(13,9)=$ fluw of undigested food from the rumen, $\mathrm{kg} /$ day, see App. A

$\mathrm{F}(17,9)=$ exit of digestible forage portion from the rumen, $\mathrm{kg} /$ day, see App. A

67. $\mathrm{I}_{114}=0.65 * \mathrm{X}_{16} * 4.3 * 0.52$

$I_{14}=$ energy from microprotein, $\mathrm{kcal}$

$\mathrm{X}_{10}=$ microprotein level, grams, in rumen

68. $\mathrm{I}_{115}=\mathrm{I}_{112}+\mathrm{I}_{138}+\mathrm{I}_{114}-125^{*} \mathrm{I}_{1081}$

$I_{116}=$ net a vailable energy, kcal

$I_{113}=$ energ. $y$ from passed good, kcal

$I_{14}=$ energ. ${ }^{\prime}$ from microprotein, $\mathrm{kcal}$

$I_{112}=$ fatty acid energy, $\mathrm{kcal}$

$I_{108}=$ metalıolic biomass, $\mathrm{kg}$

69. $I_{1101}=125 * I_{1081}$

or

$=\left|I_{118}\right|$ for animals on range when respiration exceeds caloric intake 
$\mathrm{I}_{116}=$ supplementary feed required, grams

$I_{108}=$ metabolic biomass, $\mathrm{kg}$, for ith population category

$I_{118}=$ net available energy, kcal

70. $\mathrm{I}_{1171}=0.0$

$$
\mathrm{I}_{102} \geq 1.0
$$

$$
\begin{aligned}
& =3.74+10.52 * I_{103} \\
& =3.74+5.26 * I_{102} \\
& =1.5
\end{aligned}
$$$$
0.75 \leq \mathrm{I}_{102}<1.0
$$$$
0.5 \leq \mathrm{I}_{102}<0.75
$$

$0.0 \leq \mathrm{I}_{102}<0.5$

$I_{117}=$ fraction of available energy for growth, kcal

$I_{102}=$ animal weight, mature weight ratio to beef cow

where,

71. $I_{102}=X_{14} /\left(C_{38} * I_{1021}\right)$

$\mathrm{X}_{14}=$ weight, $\mathrm{kg}$ of $\mathrm{kth}$ animal category and age group $\mathrm{N}$

$\mathrm{C}_{\mathrm{w}}=$ weight of beef cattle by age $(\mathrm{W}), \mathrm{kg}$

$I_{10 \text { I }}=$ animal weights, mature weight ratio

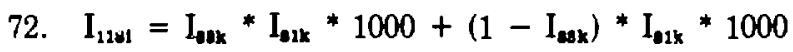

$I_{1 i \varepsilon}=$ total weight of forage consumed, grams

$I_{m}=$ cumulative green biomass uptake for kth food preference category

$I_{n}=$ ingestion rate, $\mathrm{kg} /$ day

73.

$$
I_{1111}=I_{1111} / \sum_{j=1}^{3} I_{n 2 j}
$$

$I_{119}=m^{2}$ grazed by all animal units in a given age group

$I_{114}=$ total weight of forage consumed, grams

$I_{n}=$ available forage $g / \mathrm{m}^{2}$ from all forage sources of $\mathrm{jth}$ preference category

74. $\mathrm{I}_{1901}=\mathrm{I}_{1101} * \mathrm{C}_{921} * \mathrm{I}_{1011}$

$I_{1 s 0}=m^{2}$ grazed by the ith age group of a given animal population

$\mathrm{I}_{110}=\mathrm{m}^{2}$ grazed by all animal units

$\mathrm{C}_{\mathrm{a}}=$ fraction of population hy age $(Z)$, see App. C

$I_{102}=$ product of population structure and number of animal units on range 
75. $\mathrm{I}_{1011}=\mathrm{I}_{1012} /\left(\mathrm{I}_{199} * \mathrm{C}_{24}\right)$

$I_{121}=$ fraction of area grazed by the ith age group of a given animal population

$I_{201}=$ product of population structure and number of animal units on range

$I_{192}=$ fraction of region grazed

$\mathrm{C}_{\boldsymbol{m}}=$ area of regional grid $(\mathrm{M} 7), 3.73 \times 10^{8} \mathrm{~m}^{2}$

76. $\mathrm{I}_{1911}=\left(\mathrm{I}_{741} * \mathrm{X}_{01}+\left(1-\mathrm{I}_{741}\right) * \mathrm{X}_{71} * \mathrm{X}_{08} / \mathrm{I}_{271}\right) * \mathrm{I}_{701}$

$I_{123}=\mathrm{g} / \mathrm{m}^{2}$ of green and dry standing biomass for ith plant

$I_{74}=$ fraction of diet composed of green biomass

$\mathrm{X}_{\mathrm{s}}=$ needle or leaf biomass, $\mathrm{kg} / \mathrm{m}^{2}$

$\mathrm{X}_{7}=\mathrm{g} / \mathrm{m}^{2}$ dead standing biomass

$I_{27}=g / \mathrm{m}^{2}$ live above ground biomass

$I_{78}=$ utilization efficiency, fraction of standing forage

77. $\mathrm{I}_{124 \mathrm{k}}=\left(\mathrm{I}_{1000} / \mathrm{I}_{198 \mathrm{k}}\right) *\left(\mathrm{I}_{118 \mathrm{k}}\right) *\left(\mathrm{I}_{88 \mathrm{k}}+\mathrm{I}_{87 \mathrm{k}}\right)+\left(\left(\mathrm{I}_{128 \mathrm{k}}-\mathrm{I}_{1001}\right) / \mathrm{I}_{126 \mathrm{k}}\right) *\left(\mathrm{I}_{118 \mathrm{k}}\right) *\left(\mathrm{I}_{91 \mathrm{k}}+\mathrm{I}_{92 \mathrm{k}}\right) * \mathrm{I}_{126}$

$I_{124}=$ total activity ingested from forage by lith animal category

$I_{100}=$ number of animal units on range for ith grazing interval

$I_{12 s}=$ number of animal units

$I_{114}=$ total weight of forage consumed, grams

$I_{w}=$ specific activity, activity/gram, of consumed food

$I_{n}$ = specific activity, activity/gram, on surface of consumed food

$I_{110}=$ supplementary feed required, grams

$I_{\text {g: }}=$ total activity available from supplementary feed

$I_{n}=$ total surface activity available from supplementary feed

$\mathrm{I}_{1 \mathrm{se}}=$ fraction of supplementary feed from study area

78. $\mathrm{I}_{1841}=\mathrm{I}_{1811}+\left|\mathrm{I}_{2181} / 4.3\right| * \mathrm{C}_{221} *\left(\mathrm{I}_{91 \mathrm{k}}+\mathrm{I}_{92 \mathrm{k}}\right) * \mathrm{I}_{228}$

$I_{134}=$ total activity from ingested forage available to ith age group of kth animal category

$I_{116}=$ net available energy, $\mathrm{kcal}$

$C_{u}=$ fraction of population by age $(Z)$, see App. C

$I_{\mathbf{n}_{3}}=$ total activity available from supplementary feed

$I_{m z}=$ total surface activity available from supplementary feed

$I_{120}=$ fraction of supplementary feed from study area

79. $I_{1281}=I_{110 k} *\left(\left(I_{120 k}-I_{1000}\right) / I_{128 k}\right) * C_{s 21}$

$I_{12 s}=$ supplementary feed required by ith age category of kth animal type

$I_{110}=$ supplementary feed required, grams

$I_{100}=$ number of animal units on range for jth grazing interval

$\mathrm{C}_{\boldsymbol{g}}=$ fraction of population by age $(\mathrm{Z})$, see App. C 
80. $I_{1881}=I_{1801}+I_{1009} *\left|I_{1101} / 4.3\right|^{*} C_{s 8 x}$

$\mathrm{I}_{125}=$ supplementary feed increment required by ith age category of kth animal type

$I_{100}=$ number of animal units on range for jth grazing interval

$I_{116}=$ net available energy, kcal

$\mathrm{C}_{32}=$ fraction of population by age $(\mathrm{Z})$, see App. C

81. $\mathrm{I}_{128 \mathrm{k}}=0$

$I_{\lrcorner_{\mathrm{s}}}=0$

$$
\begin{array}{ll}
=\left(\mathrm{I}_{80} / \mathrm{I}_{78 \mathrm{~h}}\right) *{ }_{-1 \mathrm{10k}} * 3 & \mathrm{~L}_{6}=1, \mathrm{I}_{27}+\mathrm{X}_{7}<20 \\
=\left(\mathrm{I}_{80} / \mathrm{I}_{78 \mathrm{k}}\right) * \mathrm{X}_{10 \mathrm{k}} * 20 /\left(\mathrm{I}_{27}+\mathrm{X}_{7}\right) & \mathrm{L}_{8}=1, \mathrm{I}_{27}+\mathrm{X}_{7} \geq 20
\end{array}
$$

$\mathrm{I}_{12 \mathrm{a}}=$ soil ingestion, grams/day by kth animal category

$\mathrm{I}_{\mathrm{B0}}=$ uptake, $\mathrm{kg} /$ day, from ith food preference category

$I_{79}=$ total sum of plants utilized by kth animal category

$\mathrm{X}_{14}=$ biomass, $\mathrm{kg}$, of animal unit

$\mathrm{I}_{27}=\mathrm{g} / \mathrm{m}^{2}$ live above ground biomass

$\mathrm{X}_{7}=\mathrm{g} / \mathrm{m}^{2}$ of dead standing biomass

$\mathrm{L}_{\mathrm{a}}=$ distinguishes woody plants $(0)$ from herb type(1)

82. $I_{1801}=\left(0.07 * X_{16}\right)^{0.04}$

$I_{1301}=$ blood weight, $\mathrm{kg}$, for $\mathrm{kth}$ population category, lth animal category

$\mathrm{X}_{14}=$ biomass, $\mathrm{kg}$, of animal unit

83. $\mathrm{I}_{\mathrm{Bs}_{1}}=\mathrm{k} * \mathrm{C}_{\mathrm{sil}}, \quad \mathrm{k}=2$

$\mathrm{C}_{83}=$ size of diameter class intervals, inches, for ith plant type

$\mathrm{I}_{182_{1}}=$ size of first diameter class, $\left(0-\mathrm{I}_{181_{1}}\right)$ inches,

84. $\mathrm{I}_{1841}=0.142 * \mathrm{I}_{271} * \mathrm{C}_{291} *\left(1-\mathrm{I}_{671} / 2\right) /\left(\mathrm{I}_{371} * \mathrm{I}_{881}\right)-\mathrm{I}_{1861}$

$I_{1841}=$ growth increment, $\mathrm{ft}^{\mathrm{s}} / \mathrm{yr}$, for specific plant in a given year

$I_{27}=\mathrm{g} / \mathrm{m}^{2}$ live above ground biomass

$\mathrm{C}_{2 \mathrm{~g}}=$ taper coefficient (V8), see App. C

$I_{47}=$ fractional crown cover

$I_{37}=$ succession factor

$I_{3 \mathrm{e}}=$ daily structural biomass competition factor, $\mathrm{g} / \mathrm{m}^{2}$

$I_{196}=$ virtual stand volume, $\mathrm{ft}^{3}$, for setting radial growth rate of boles

85. $\mathrm{I}_{1351}=0.142 * \mathrm{I}_{271} * \mathrm{C}_{291} *\left(1-\mathrm{I}_{477} / 2\right) /\left(\mathrm{I}_{371} * \mathrm{I}_{381}\right)$

$I_{196}=$ virtual volume, $\mathrm{ft}^{\mathrm{s}}$, required for setting radial growth rate of stand boles for ith plant

$\mathrm{I}_{27}=\mathrm{g} / \mathrm{m}^{2}$ live above ground biomass

$\mathrm{C}_{29}=$ taper coefficient (V8), see App. C

$\mathrm{I}_{47}=$ fractional crown cover

$I_{37}=$ succession factor

$\mathrm{I}_{\mathrm{se}}=$ daily structural biomass competition factor, $\mathrm{g} / \mathrm{m}^{2}$ 
86. $I_{197 k}=I_{14+k} / 3$

$$
=\mathrm{I}_{14 \mathrm{kk}} * 77.3 / \mathrm{I}_{1 \mathrm{mo}}
$$

$\mathrm{I}_{197}=$ volume, $\mathrm{ft}^{3}$, of $\mathrm{kth}$ diameter class for ith plant

$\mathrm{I}_{1 \mathrm{u}}=$ wood of stand, $\mathrm{ft}^{*}$

$\mathrm{I}_{144}=$ volume of average tree, $\mathrm{ft}^{3}$

87. $\mathrm{I}_{1881}=\mathrm{I}_{140 \mathrm{k}} *\left(\mathrm{I}_{1801} * \mathrm{I}_{1411}+\mathrm{C}_{289} / 2\right)^{2} *\left(\mathrm{I}_{1801}\right)^{2}$

$I_{128}=$ total advantage factor for kth diameter class of ith plant

$I_{160}=$ number of trees/acre

$I_{100}=$ class diameter index, inches

$I_{141}=$ bole to crown conversion factor

$\mathrm{C}_{20}=$ taper coefficient (V8), see App. C

88. $\mathrm{I}_{140 \mathrm{k}}=\mathrm{X}_{20 \mathrm{k}} / \mathrm{I}_{144 \mathrm{k}}$

$I_{140}=$ number of trees/acre for kth diameter class of given plant

$\mathrm{X}_{\mathrm{20}}=$ volume, $\mathrm{ft}^{\mathrm{s}}$

$I_{144}=$ volume of average tree, $\mathrm{ft}^{\mathrm{s}}$

89.

$$
\mathrm{I}_{243 \mathrm{k}}=\mathrm{I}_{1341}{ }^{*} \mathrm{I}_{288 \mathrm{k}} / \sum_{\mathrm{k}=2}^{\mathrm{n}} \mathrm{I}_{18 \mathrm{k} \mathrm{k}}
$$

$I_{162}=$ growth allocation factor, $\mathrm{ft}^{\mathrm{t}}$, for kth diameter class of ith plant

$\mathrm{I}_{\mathbf{1 8 4}}=$ growth increment, $\mathrm{ft} \% / \mathrm{yr}$

$I_{188}=$ total advantage factor for biomass allocation

90.

$$
I_{1481}=I_{1861}-\sum_{i=1}^{n} X_{20 k}
$$

$I_{148}=$ volume difference between stand generated volume and calculated volume of ith plant $I_{186}=$ wood of stand, $\mathrm{ft}^{\mathrm{s}}$

$\mathrm{X}_{20}=$ volume, $\mathrm{ft}^{\circ}$ of $\mathrm{kth}$ diameter class

91. $\left.\mathrm{I}_{144 \mathrm{k}}=0.5 * 0.035 * \mathrm{I}_{1 \mathrm{4B1}} / \mathrm{C}_{291}\right) *(2 * 24 * 2.54)^{2}$

$$
i=1
$$

$$
=(0.035) *\left(I_{1881} / C_{201}\right) *\left[\left(i *\left(I_{1821}-1 / 2\right)\right)^{2}+\left(i *\left(I_{1821}+1 / 2\right)\right)^{2} l / 2\right.
$$

$I_{144}=$ volume of average tree, $\mathrm{ft}^{\mathrm{a}}$ of $\mathrm{kth}$ diameter class of ith plant

$I_{165}=$ specific gravity of wood

$\mathrm{I}_{132}=$ size of diameter class intervals

$\mathrm{C}_{2 p}=$ taper coefficient (V8), see App. C 
92.

$I_{1481}=\sum_{k=1}^{n} X_{20 k}$

$\mathrm{I}_{149}=$ cumulative total activity, activity/gram, of total stand for ith plant type

$\mathrm{X}_{20}=$ volume. $\mathrm{ft}^{\mathrm{3}}$ of kth diameter class

93. $\mathrm{I}_{180 \mathrm{k}}=\left(\mathrm{X}_{20 \mathrm{k}}\right)_{\mathrm{p}} /\left(\mathrm{X}_{20 \mathrm{k}} / 0.142\right)$

$I_{1 s 0}=$ specific activity, activity/gram, for kth diameter class of given plant

$\mathrm{X}_{20}=$ volume, $\mathrm{ft}^{\mathrm{s}}$

$\mathrm{p}=\mathrm{TRU}$ pool material

94. $\mathrm{I}_{160 \mathrm{k}}=\mathrm{I}_{180 \mathrm{k}}-\mathrm{I}_{80_{\mathrm{k}-1}}$

$\mathrm{I}_{\mathrm{Bb}}=$ specific activity, activity/gram, for $\mathrm{kth}$ diameter class of a given plant

95. $\mathrm{I}_{\mathrm{it5j}}=\mathrm{I}_{86 \mathrm{j}} *\left(\mathrm{X}_{8 \mathrm{j}} * \mathrm{I}_{7 \mathrm{j}}+\left(1-\mathrm{I}_{7 \mathrm{j}}\right) * \mathrm{X}_{7 \mathrm{~J}} * \mathrm{X}_{8 \mathrm{~J}} / \mathrm{I}_{27 \mathrm{j}}\right) * \mathrm{I}_{78 \mathrm{~J}}$

$I_{161}=$ sum of total nitrogen concentration $\times 100$ for jth plant type

$\mathrm{I}_{86}=$ nitrogen content, percentage, of consumed biomass

$\mathrm{X}_{\mathrm{b}}=$ needle or leaf biomass, $\mathrm{kg} / \mathrm{m}^{2}$

$\mathrm{X}_{7}=\mathrm{g} / \mathrm{m}^{2}$ of dead standing biomass

$I_{74}=$ fraction of diet composed of green biomass

$I_{27}=\mathrm{g} / \mathrm{m}^{2}$ live above ground biomass

$I_{75}=$ utilization efficiency, fraction of standing green forage

96. $\mathrm{l}_{1621}=30 * \mathrm{I}_{781}$

$I_{162}=$ water content, percentage, of consumed biomass for ith plant type

$I_{78}=$ nitrogen content, percentage, of standing green biomass

97. $I_{18 s}=0.456$

$I_{821} \geq 1.8$

$=0.023^{*}\left(\mathrm{I}_{821}-0.6\right)+0.0186$

$0.6 \leq \mathrm{I}_{321}<1.8$

$=0.031 * \mathbf{I}_{\mathbf{8 2 1}}$

$0 \leq I_{821}<0.6$

$I_{138}=$ grams $/ \mathrm{kcal}$ of microprotein in digestible fraction

$\mathrm{I}_{\mathrm{B2}}=$ nitrogen content of ingested forage, $\%$

\section{EQUATIONS INVOLVING DRIVING VARIABLES}

1. $D_{2}=\left[\left(I_{7}-I_{8} / 2\right]^{*}\left[\sin \left(2 \pi^{*}\left(I_{5}-I_{9}\right) / 365\right)+1\right]+I_{8}\right.$

$\mathrm{D}_{2}=$ daily temperature, ${ }^{\circ} \mathrm{C}$

$\mathrm{I}_{7}=$ maximum monthly temperature, ${ }^{\circ} \mathrm{F}$, average on daily basis

$I_{6}=$ day number

$\mathrm{I}_{9}$ = lag period to maximum temperature 
2. $D_{2}=D_{2}+I_{10}^{*}\left(I_{11}-I_{12}\right)+I_{10}$

$\mathrm{D}_{2}=$ daily temperature, ${ }^{\circ} \mathrm{C}$

$I_{10}=$ lapse rate, ${ }^{\circ} \mathrm{F} / \mathrm{ft}$ rise from reference site

$I_{11}=$ reference elevation, feet above sea level

$I_{12}=$ elevation of site, feet above sea level

$I_{1 s}=$ latitude relative to reference site

3. $\mathrm{D}_{2}=\left(\mathrm{D}_{2}-32\right) * 5 / 9+\mathrm{S}_{\mathrm{a}} * \mathrm{I}_{14}$

$\mathrm{D}_{2}=$ daily temperature, ${ }^{\circ} \mathrm{C}$

$S_{8}=$ random number drawn from a normal distribution $(\mathrm{m}=0, \sigma=1$ )

$\mathrm{I}_{14}=$ maximum temperature variation, ${ }^{\circ} \mathrm{F}$, on a monthly basis

4. $\left.\mathrm{D}_{3}=\left|\left(\mathrm{I}_{24}-\mathrm{I}_{28}\right) / 2\right| * \mid \sin \left(2 \pi^{*}\left(\mathrm{I}_{8}-\mathrm{I}_{28}\right) / 365\right\}+1\right\}+\mathrm{I}_{2 \mathrm{~b}}$

$D_{s}=$ solar radiation, Ly/day, on day $I_{s}$

$I_{24}=$ maximum solar radiation, $L y /$ day, received during the year

$I_{25}=$ minimum solar radiation, Ly/day, received during the vear

$\mathrm{I}_{2 g}=$ lag period to maximum insolation, days

$I_{B}=$ day number

\section{EQUATIONS INVOLVING LOGIC AND BOUNDARY VARIABLES}

1. $\mathrm{I}_{1}=\mathrm{F}(\mathrm{P}, 1)$

$$
=F(P, 2)
$$

$\mathrm{L}_{1}=$ temperature greater than $0^{\circ} \mathrm{C}$

$F^{\prime}(P, 1)=$ transport of water from precipitation events to soil

$\mathrm{F}(\mathrm{P}, 2)=$ transport of water from snow to soil

$\mathrm{D}_{2}=$ daily temperature, ${ }^{\circ} \mathrm{C}$

2. $\mathrm{L}_{\text {s01 }} \leq 733^{*}\left(\mathrm{I}_{1821} / 2\right) *\left(1-0.5^{*} \mathrm{I}_{171}\right)=\mathrm{I}_{1881}$

$\grave{\mathrm{L}}_{10 \mathrm{~s}}=$ virtual stand volume, $\mathrm{ft}^{\mathrm{g}}$, greater than minimum volume for given biomass density required for Forman access for ith plant category

$I_{182}=$ size of diameter class intervals

$I_{47}=$ fractional crown cover for specific plant

$\mathrm{I}_{183}=$ minimum volume, $\mathrm{ft}^{\mathrm{a}}$, for given biomass density which is required for Forman access

\section{EQUATIONS INVOLVING STOCHASTIC VARIABLES}

1.

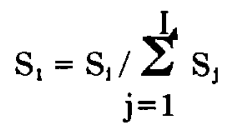

$\mathrm{S}_{1}=$ random number drawn from a normal distribution $(\mathrm{m}=0, \sigma=1)$

$\mathrm{I}_{4}=$ total rainfall events/month totaling more than $0.1 "$ precipitation

$\mathrm{S}_{\mathrm{J}}=$ stochastic variables, see App. C 


\section{EQUATIONS INVOLVING STATE VARIABLES}

1. $X_{1}=X_{1}+25.4 * I_{891}$

$$
\begin{aligned}
& I_{8}=I_{62 \mathrm{k}} \\
& I_{s}=I_{62 \mathrm{k}}
\end{aligned}
$$$$
=x_{1}
$$

$X_{1}=$ current value of soil moist ure availability

$\mathrm{I}_{\theta 3}=$ succession ranking according to dominance for ith plant

$I_{\mathbf{g} 2}=$ harvesting scheduies, day number

$I_{6}=$ day number

2. $X_{6}=0.928 * I_{27} * \operatorname{EXP}\left(-3.11 * I_{27}\right)+0.072 * I_{27}$

$\mathrm{X}_{6}=$ needle or leaf' biomass, $\mathrm{kg} / \mathrm{m}^{2}$

$\mathrm{I}_{27}=\mathrm{g} / \mathrm{m}^{2}$ live above ground biomass

3. $\mathrm{X}_{5}=\mathrm{C}_{16} * \mathrm{I}_{28}$

$$
\begin{aligned}
& X_{5} \geq C_{15} * I_{28} \\
& X_{5}<C_{18} * I_{28}
\end{aligned}
$$

$=\mathrm{X}_{0}$ (previous calculation)

$\mathrm{X}_{6}=$ needle or leaf biomass, $\mathrm{kg} / \mathrm{m}^{2}$

$\mathrm{I}_{2 \mathrm{~B}}=$ running average productivity, $\mathrm{kg} / \mathrm{m}^{2} / \mathrm{yr}$

$\mathrm{C}_{15}=$ leaf biomass allocation, see App. C

4. $\mathrm{X}_{0}=\mathrm{X}_{0}$

$$
\begin{aligned}
& \mathrm{L}_{4}=1 \\
& \mathrm{~L}_{4}=0, \mathrm{I}_{81}+1<\mathrm{I}_{8}<\mathrm{I}_{32}+1 \\
& \mathrm{~L}_{4}=0, \mathrm{I}_{91}+1>\mathrm{I}_{5}>\mathrm{I}_{32}+1
\end{aligned}
$$$$
=\mathrm{X}_{5}
$$$$
=1.0
$$

$\mathrm{X}_{5}=$ needle or leaf biomass, $\mathrm{g} / \mathrm{m}^{2}$

$\mathrm{L}_{4}=$ determines whet her leaf fall occurs at end of frost free period (0) or if evergreen (1)

$I_{31}=$ beginning of frost-free period, day number

$I_{32}=$ end of frost-free period, day number

$I_{5}=$ day number

5. $\quad \mathrm{X}_{161}=200 * \mathrm{C}_{391}$

$X_{16}=$ microprotein level, grams, in rumen for it h age group of a given animal type $C_{y_{3}}=$ initial weight of animal unit by age $\mathrm{kg}$, see App. $\mathrm{C}$

6. $\mathrm{X}_{201}=\mathrm{I}_{1391} * \operatorname{EXP}\left(-1 / \mathrm{C}_{271}\right) *\left(\mathrm{I}_{1981}-\mathrm{I}_{1931}\right)$

$$
\begin{aligned}
& X_{20_{1}}>I_{137_{1}} \\
& X_{20_{1}} \leq I_{137_{1}}
\end{aligned}
$$$$
=0
$$

$\mathrm{X}_{2 \mathrm{i}}=$ volume, $\mathrm{ft}^{3}$, of the first diameter class of ith plant

$I_{133}=$ minimurn volume for given biomass density required for Forman access

$C_{27}=$ mean average life-span, yrs, see App. $C$

$\mathrm{I}_{136}=$ virtual stand volume, $\mathrm{ft}^{3}$, required for setting radial growth rate of stand boles

$\mathrm{I}_{137}=$ minimum volume, $\mathrm{ft}^{3}$, of first diameter class required for estimation of $\mathrm{X}_{\mathbf{2 0}}$ 
7. $\mathrm{X}_{200_{3}}=\mathrm{I}_{1201}-\mathrm{X}_{20_{1}}$

$\mathbf{X}_{\mathbf{2 0}}=$ volume, $\mathrm{ft}^{\mathbf{2}}$, of second diameter class of ith plant category

$\mathrm{I}_{120}=$ virtual stand volume, $\mathrm{ft}^{\mathrm{t}}$, required for setting radial growth of stand boles

8. $\mathrm{X}_{20 \mathrm{k}}=\mathrm{X}_{20 \mathrm{k}} * \mathrm{I}_{12 \mathrm{a} /} / \mathrm{I}_{1231}$

$\mathrm{X}_{20}=$ volume, $\mathrm{ft}^{\mathrm{p}}$, for kth diameter of ith plant category

$I_{\mathrm{ab}}=$ virtual stand volume, $\mathrm{ft}^{\mathrm{s}}$, required for setting radial growth of stand boles

$\mathrm{I}_{\mathrm{ase}}=$ wood of stand, $\mathrm{ft}^{\mathrm{s}}$

9. $\mathrm{X}_{20 \mathrm{k}}=\mathrm{X}_{20 \mathrm{k}} *\left(\mathrm{I}_{1083} /\left(\mathrm{X}_{5}+\mathrm{X}_{6}\right)_{1}\right.$

$\mathrm{X}_{20}=$ volume, $\mathrm{ft}^{\mathbf{2}}$, for kth diameter of ith plant category

$I_{1 a s}=$ total activity, activity/gram, of total stand, cumulative

$X_{4}=g / m^{2}$ live above ground biomass

$\mathrm{X}_{0}=$ needle or leaf biomass, $\mathrm{kg} / \mathrm{m}^{2}$

10. a) $\left(\mathrm{X}_{\mathbf{4}}+\mathrm{X}_{8}\right)=0.0$

b) $\mathrm{X}_{\mathrm{s}}=0.0$

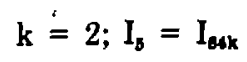

c) $\left(\mathrm{X}_{4}+\mathrm{X}_{\mathrm{B}}\right)=\left(\mathrm{X}_{4}+\mathrm{X}_{8}\right) *\left(20 / \mathrm{I}_{27}\right)$

$$
I_{b}=I_{82, k} ; 2 \leq k \leq 8, I_{27}>20.0
$$

d) $X_{s}=X_{b}^{*}\left(20 / I_{27}\right)$

$\mathrm{X}_{\mathrm{a}}=\mathrm{g} / \mathrm{m}^{2}$ live root biomass

$\mathrm{X}_{\mathbf{4}}=\mathrm{g} / \mathrm{m}^{2}$ live above ground biomass

$\mathrm{X}_{\mathrm{s}}=$ needle or leaf biomass, $\mathrm{kg} / \mathrm{m}^{2}$

$\mathrm{I}_{\mathrm{s}}=$ day number

$I_{37}=\mathrm{g} / \mathrm{m}^{2}$ live above ground biomass

$I_{02}=$ harvesting schedules, day number

$I_{04}=$ harvesting schedules, day number 


\section{APPENDIX C}

VARIABLES AND THEIR MNEMONIC EQUIVALENTS

Intermediate Variables Listing (li)

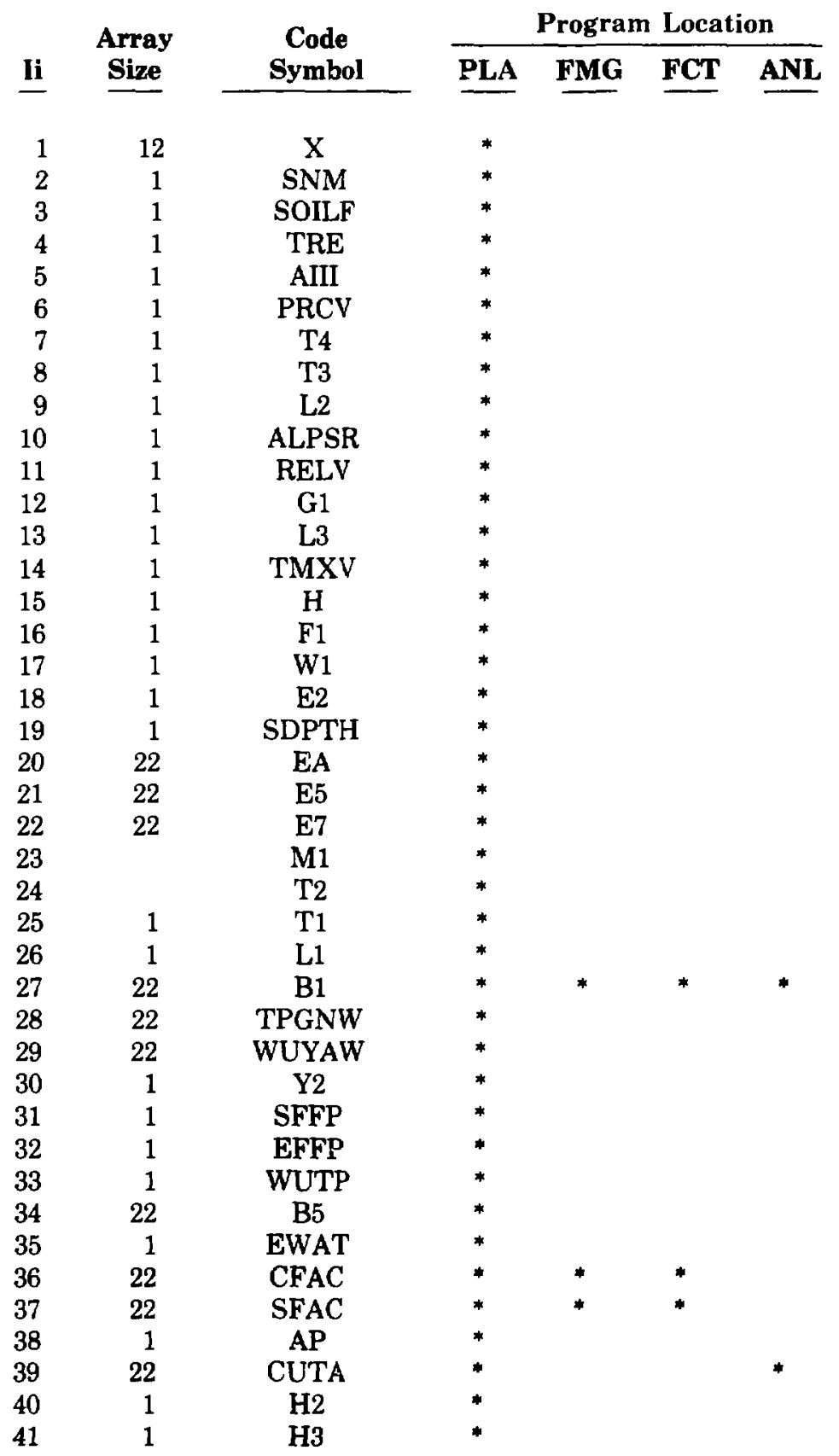


Intermediate Variables Listing (Ii) (cont)

\begin{tabular}{|c|c|c|c|c|c|c|}
\hline \multirow[b]{2}{*}{$\mathbf{I i}$} & \multirow{2}{*}{$\begin{array}{c}\text { Array } \\
\text { Size }\end{array}$} & \multirow{2}{*}{$\begin{array}{c}\text { Code } \\
\text { Symbol }\end{array}$} & \multicolumn{4}{|c|}{ Program Location } \\
\hline & & & PLA & FMG & FCT & ANL \\
\hline 42 & 1 & RSUSP & $*$ & & & \\
\hline 43 & 1024 & AMAC & $*$ & & & \\
\hline 44 & 1 & AIRCA & * & & & \\
\hline 45 & 1200 & AA & & * & * & \\
\hline 46 & 1 & A11 & * & & & \\
\hline 47 & 22 & CCVV & $*$ & * & * & \\
\hline 48 & 365 & $\mathbf{P}$ & $*$ & & & \\
\hline 49 & 22 & TLAI & * & & & \\
\hline 50 & 1 & BP & * & & & \\
\hline 51 & 704 & $\mathrm{Z1}$ & * & & & * \\
\hline 52 & 704 & $\mathrm{ZU}$ & * & & & \\
\hline 53 & 704 & $\mathrm{ZF}$ & * & & & \\
\hline 54 & 1 & IICDE & $*$ & & & \\
\hline 55 & 1 & RPNTP & $*$ & & & \\
\hline 56 & 22 & STBR & * & & & \\
\hline 57 & 704 & $\mathrm{ZF}$ & * & & & \\
\hline 58 & 1 & $\mathrm{ADD}$ & * & & & \\
\hline 59 & 1 & $\mathrm{~B} 111+\mathrm{R} 111$ & $*$ & & & \\
\hline 60 & 22 & $1 \mathrm{~B} 71$ & $*$ & & & \\
\hline 61 & 336 & DIRA & * & & & \\
\hline 62 & & $\operatorname{CUTA}\left(I_{29}\right)$ & $*$ & & & \\
\hline 63 & & $\operatorname{CUTA}\left(I_{90}\right)$ & 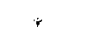 & & & \\
\hline 64 & & $\operatorname{CUTA}\left(I_{28}\right)$ & *. & & & \\
\hline 65 & & $\operatorname{DIRA}\left(\mathrm{I}_{81}\right)$ & * & & & \\
\hline 66 & 22 & CNIT & & & & * \\
\hline 67 & & $\operatorname{SFAC}\left(\mathbf{I}_{37}\right)$ & & & & \\
\hline 68 & & $\operatorname{SFAC}\left(I_{37}\right)$ & * & & & \\
\hline 69 & 1 & STBMX & * & & & \\
\hline 70 & 88 & PUTZD & & & & * \\
\hline 71 & 3 & QFRAC & & & & $*$ \\
\hline 72 & 3 & QSUM & & & & * \\
\hline 73 & 4 & SPUT & & & & * \\
\hline 74 & 22 & $\mathrm{G}$ & & & & * \\
\hline 75 & 22 & A & & & & * \\
\hline 76 & 22 & P4 & & & & * \\
\hline 77 & 22 & PNIT & & & & $*$ \\
\hline 78 & 22 & SNIT & & & & $*$ \\
\hline 79 & 22 & PP6 & & & & $*$ \\
\hline 80 & 3 & UP & & & & $*$ \\
\hline 81 & 1 & 0 & & & & $*$ \\
\hline 82 & 1 & $\mathbf{N}$ & & & & $*$ \\
\hline 83 & 1 & GA & & & & * \\
\hline 84 & 3 & GTDR & & & & $*$ \\
\hline 85 & 8 & SPAC & & & & * \\
\hline 86 & 3 & SACT & & & & * \\
\hline 87 & 8 & SPAR & & & & $*$ \\
\hline
\end{tabular}


Intermediate Variables Listing (Ii) (cont)

\begin{tabular}{|c|c|c|c|c|c|c|}
\hline \multirow[b]{2}{*}{$\underline{\text { Ii }}$} & \multirow{2}{*}{$\begin{array}{c}\text { Array } \\
\text { Size }\end{array}$} & \multirow{2}{*}{$\begin{array}{c}\text { Code } \\
\text { Symbol }\end{array}$} & \multicolumn{4}{|c|}{ Program Location } \\
\hline & & & PLA & FMG & FCT & ANL \\
\hline 88 & 3 & SAIR & & & & * \\
\hline 89 & 4 & HRVST & & & & * \\
\hline 90 & 22 & ACRES & $*$ & * & * & * \\
\hline 91 & 1 & SPAS & & & & $*$ \\
\hline 92 & 1 & SPAA & & & & * \\
\hline 93 & 4 & HRVSP & & & & * \\
\hline 94 & 1 & SPAP & & & & * \\
\hline 95 & 1 & SAAP & & & & $*$ \\
\hline 96 & 1 & SPAS & & & & * \\
\hline 97 & 1 & SPAA & & & & $*$ \\
\hline 98 & 1 & $\mathrm{AlTT}$ & * & & & * \\
\hline 99 & 22 & $\mathrm{Z1}$ & * & * & * & * \\
\hline 100 & 1 & HERD & & & & * \\
\hline 101 & 8 & Z*HERD & & & & * \\
\hline 102 & 1 & W3 & & & & $*$ \\
\hline 103 & 32 & $\mathbf{M}$ & & & & * \\
\hline 104 & 32 & $\mathbf{R}$ & & & & $*$ \\
\hline 105 & 1 & DDI & & & & $*$ \\
\hline 106 & 1 & $\mathbf{H}$ & & & & * \\
\hline 107 & 1 & H1 & & & & $*$ \\
\hline 108 & 1 & $\mathrm{~F} 2$ & & & & $*$ \\
\hline 109 & 1 & $\mathrm{E}$ & & & & * \\
\hline 110 & 32 & $\mathrm{~S}$ & & & & $*$ \\
\hline 111 & 1 & $\mathrm{X} 1$ & & & & $*$ \\
\hline 112 & 1 & $\mathrm{BB}$ & & & & $*$ \\
\hline 113 & 1 & $\mathrm{E} 1$ & & & & $*$ \\
\hline 114 & 1 & $\mathrm{E} 2$ & & & & * \\
\hline 115 & 1 & $\mathrm{E} 4$ & & & & * \\
\hline 116 & 1 & GSUPF & & & & $*$ \\
\hline 117 & 1 & E5 & & & & $*$ \\
\hline 118 & 1 & $\mathrm{C} 2$ & & & & * \\
\hline 119 & 1 & $\mathrm{C} 4$ & & & & $*$ \\
\hline 120 & 1 & M5 & & & & * \\
\hline 121 & 1 & M6 & & & & * \\
\hline 122 & 4 & $\mathrm{AA} 2$ & & & & $*$ \\
\hline 123 & 22 & TSUMB & & & & * \\
\hline 124 & 1 & $\mathrm{C} 3$ & & & & * \\
\hline 125 & 4 & N5 & & & & $*$ \\
\hline 126 & 4 & FSUPF & & & & * \\
\hline 127 & 4 & TOTSF & & & & $*$ \\
\hline 128 & 1 & C5 & & & & * \\
\hline 129 & 32 & $Y$ & & & & * \\
\hline 130 & 32 & $\mathrm{X}$ & & & & $*$ \\
\hline 131 & 4 & P5 & & & & * \\
\hline 132 & 22 & $\mathrm{XJ1}$ & & & & \\
\hline 133 & 1 & $\mathrm{~T} 2$ & & $*$ & & \\
\hline 134 & 1 & G4 & & * & & \\
\hline
\end{tabular}




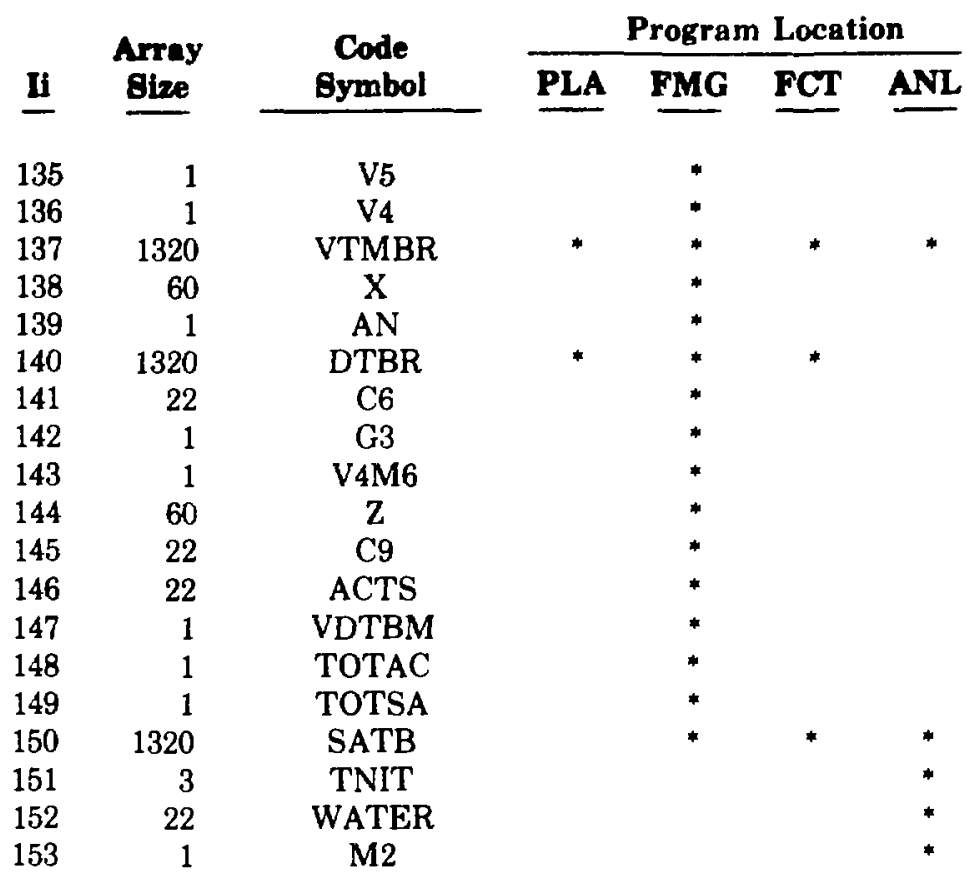

Driving Variables (Di)

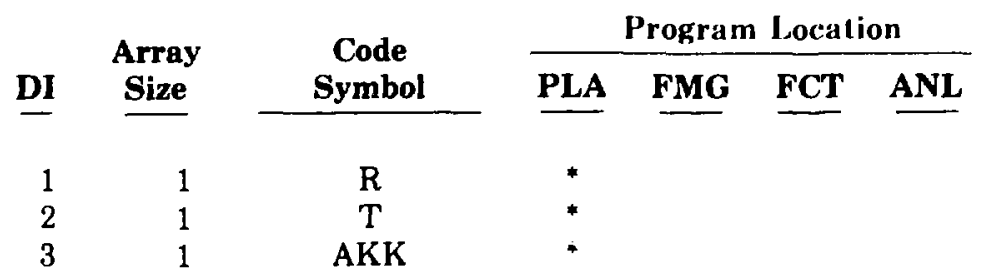

Logic and Boundary Variables (Li)

\begin{tabular}{|c|c|c|c|c|c|}
\hline \multirow{2}{*}{$\begin{array}{c}\text { Array } \\
\text { Size }\end{array}$} & \multirow{2}{*}{$\begin{array}{c}\text { Code } \\
\text { Symbol }\end{array}$} & \multicolumn{4}{|c|}{ Program Location } \\
\hline & & PLA & FMG & FCT & ANL \\
\hline 1 & $\mathrm{~T} \geq 0$ & * & & & \\
\hline 1 & $\operatorname{BAP}(0,1)$ & * & & & \\
\hline 1 & $\operatorname{BCP}(0,2)$ & $*$ & & & \\
\hline 1 & ISTP $(0,1)$ & * & & & \\
\hline 22 & $\operatorname{SCTP}(0,2)$ & $*$ & & & \\
\hline 1 & $I Y R \geq 0$ & $*$ & & & \\
\hline 1 & CHRSY $\geq 0$ & $*$ & & & \\
\hline 128 & YRGRZ:AI1 & & & & $*$ \\
\hline 128 & ONRNG(AIII) & & & & $*$ \\
\hline 1 & $\mathrm{~V} 5>\mathrm{T} 1$ & & * & . & \\
\hline
\end{tabular}


Stochastic Variables (Si)

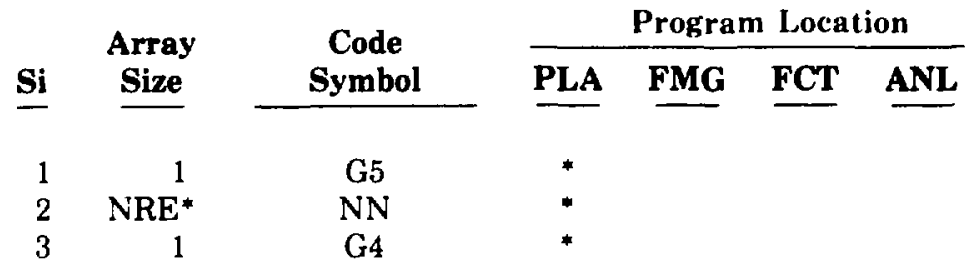

"See Variable library (App. I)

User Option Variables (Oi)

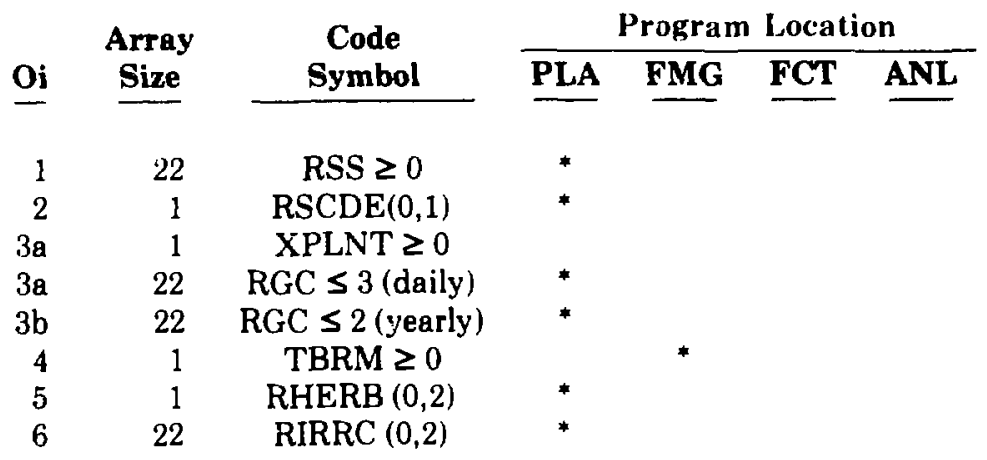

State Variable (Xi)

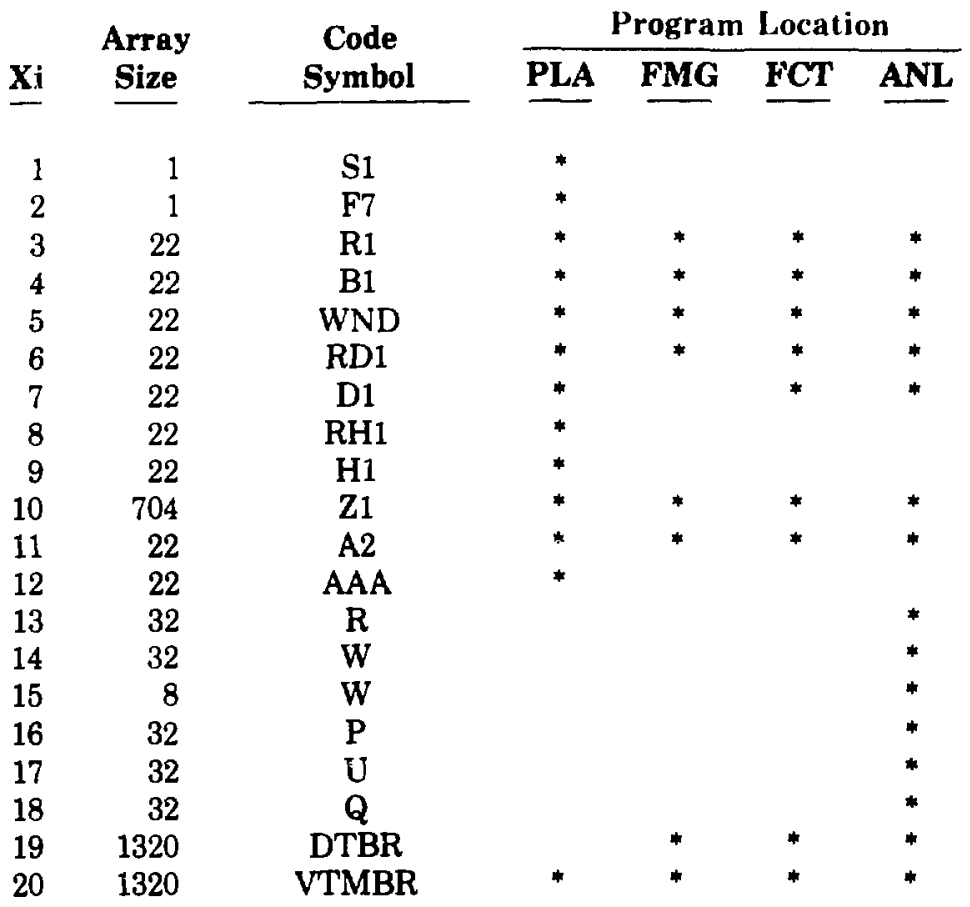




\section{Constants}

$\mathbf{C i}$

1. Snow melt coefficient

2. Evapotranspiration intercept

3. Evapotranspiration proportionality

$1.128 \mathrm{~mm} /{ }^{\circ} \mathrm{C}$

4. Soil depth adjustment

$0.6 \mathrm{~mm}$

$0.18 \mathrm{~mm} /$ day

5. Soil depth exponential adjustment

0.632

6. Optimum growth temperature

0.004

$25\left(1,{ }^{*} 2,3,5,7,8\right)$

$18(12,13,15,18,19)$

$21(4,6,9,10,11)$

$15(16,20,21)$

$14(14)$

12 (17)

7. Effect of tempr:ature, above optimum

${ }^{\circ} \mathrm{C}$

$81(1,2,3,5,7,8)$

$169(4,6,9,10,1 ;)$

$256(12,13,15,18,19)$

$400(14)$

$484(17)$

$361(16,20,21)$

8. Effect of temperature, below optimum

${ }^{\circ} \mathrm{C}^{2}$

$225(1,2,3,7,8)$

$169(12,13,18,19)$

$121(4,6,10,11)$

$625(5)$

$441(9)$

$196(14)$

$324(15)$

$100(16,21)$

$144(17)$

$225(20)$

9. Effect of soil moisture on photosynthesis

$5.0(1,2,3,4,5,6,7,8,9)$

3. $0(10,12,13,14,15,16,17,18,19,20,21)$

4.0 (11)

10. Effect of solar radiation on photosynthesis

11. Respiratory maintenance component (ARP)

$0.001 /$ Ly/day

$0.0(1,2,3,7,8,11,12,13,18,19)$

$1.5(4,10,21)$

$2.0(6,14,16)$

$3.0(15,17,20)$

$5.0(5,9)$

12. Respiratory effect 1

$0.01(1,2,3,4,5,6,7,8,9,10,11,15)$

$0.03(12,13,14,16,17,18,19,20,21)$

13. Respiratory effect 2

$0.161(1,2,3,4,5,6,7,8,9,10,11)$

$0.153(15)$

$0.120(12,13,14,16,17,18,19,20,21)$

*Plant numbers, see variable library. 


\section{Constants (cont)}

Ci

14. Respiratory effect 3

$0.13(1,2,3,4,5,6,7,8,9,10,11)$

$0.20(15)$

$0.18(12,13,14,16,17,18,19,20,21)$

15. Leaf biomass allocation

$1.0(5,9,14,15,17,20)$

$0.5(4,6,10,16,21)$

16. Minimum water utilization efficiency (WUTP) $\mathrm{ml} / \mathrm{g}$

$350(1,2,3,4,7,8,11,12,13,18,19)$

$725(5,9,14,15,17,20)$

$900(6,10,16,21)$

17. Hatch-slack coefficient (PSE)

$0.5(1)$

1.0 (all other plants)

18. Fraction of photosynthate transferred to roots (RTRANS)

$0.7(3,8,13,18)$

$0.25(1,2,6,9,12,16,20)$

$0.15(4,5,10,14,15,17,21)$

$0.5(7,11,19)$

19. Fraction of photosynthate remaining above ground (STRANS)

$0.3(3,8,13,18)$

$0.75(1,2,6,9,12,16,20)$

$0.85(4,5,10,14,15,17,21)$

$0.5(7,11,19)$

20. Mortality factor 1

$0.05(1,2,11,12)$

$0.10(3,7,8,13,17,18,19)$

21. Mortality temperature effect

${ }^{\circ} \mathrm{C}$

$25(1,2,3,7,8)$

$21(11,12)$

$18(13,18,19)$

22. Mortality factor 2

$0.1(1,2,3,7,8,11,12,13,18,19)$

23. Mortality factor 3

$1.1(1,2,3,7,8,11,12,13,18,19)$

24. Mortality factor 4

$0.1(1,2,3,7,8)$

$0.14(11, \mathrm{i} 2)$

$0.2(13,18,19)$

25. Mortality factor 5

$0.1(1,2,12)$

26. Mortality temperature factor, lower

$10(1,2,3,7,8)$

$7(11,12)$

$5(13,18,19)$

27. Mean average life-span (HF)

$1(1,2,7,12,19)$

$30(3,8,11,13,18)$

$70(6,9,16,20)$

$150(4)$

$175(10,21)$

$300(5,14,15,17)$ 
28. Initial activity in atmosphere (CHRAC)

$\mathrm{ACT} / \mathrm{m}^{2}$

29. Taper coefficient (V8)

$1.0(1,2,3,4,6,7,8,9,10,11,12,12,14,16,18,19,20,21)$

$0.8(17)$

$1.36(15)$

$2.5(5)$

30. Effective succession life-r.pan (RSCF)

$\mathrm{C}_{\boldsymbol{2}}(1,2,3,4,5,6,8,9,10,11,12,13,14,15,16,17,18,20,21)$

$9.0(19,7)$

51. Nitrogen content of dry forage (N3)

$0.6 \%$

32. Fraction of population by age (Z)

Animals 1,2 (0.4,0.06,0.12,0.18,0.12,0.06,0.036,0.024)

Animals 3,4 (0.25,0.23,0.16,0.12,0.12,0.05,0.05,0.02)

33. Initial weight of beef cattle by age (W)

$1-8$ yrs $(23,290,410,494,550,550,550,550)$

34. Area of regional grid (M7)

kg

35. Diameter class intervals

$3.73 \times 10^{1} \mathrm{~m}^{2}$

$(0.2 ; 2)$ inches 


\section{APPENDIX D}

\section{VARIABLE LIBRARY}

\begin{tabular}{|c|c|c|}
\hline Symbol & Location" & Definition \\
\hline A & 1 & Mid range solar radiation value Ly/day \\
\hline $\mathbf{A}(22)$ & 4 & $\begin{array}{l}\text { Utilization efficiency, fraction of standing } \\
\text { forage }\end{array}$ \\
\hline AA & 2,3 & Call statement (see Main Program, AAA(l) \\
\hline $\operatorname{AAA}(22)$ & 1 & $\begin{array}{l}\text { Activity } / \mathrm{m}^{2} \text { from fire increment to total air } \\
\text { activity }\end{array}$ \\
\hline AAKK & 1 & $\begin{array}{l}\text { Counter for plant types included in succession } \\
\text { module }\end{array}$ \\
\hline $\operatorname{AATB}(22,60)$ & 2,3 & Average age of trees(l) with diameter(k) \\
\hline AA2(4) & 4 & $\begin{array}{l}\text { Input variable, fraction of region grazed by specific } \\
\text { animal unit }\end{array}$ \\
\hline $\mathrm{ABC}$ & 1 & $\begin{array}{l}\text { Difference between real value of } Q(l) \text { and integer } \\
\text { value of } Q(1)\end{array}$ \\
\hline $\mathrm{AC}$ & 1 & Root respiration factor for some plant types \\
\hline $\operatorname{ACRES}(22)$ & $1,2,3,4$ & $\begin{array}{l}v=\text { acres/plant }(i),(i=1,22 ; 0 \leq v \leq \text { grid size. For } \\
v=0, \text { grid size assumed }(v=\text { size, acres })\end{array}$ \\
\hline ACTAV & 2 & $\begin{array}{l}\text { Geometric mean of stand, specific activty, } \\
\text { activity/gram }\end{array}$ \\
\hline ACTS & 2 & $\begin{array}{l}\text { Logarithmic sum of class diameter specific } \\
\text { activity }\end{array}$ \\
\hline $\operatorname{ACUT}(22,2)$ & 3 & $\begin{array}{l}\text { Input variable, lower \& upper diameters selected } \\
\text { for cutting in "A" class cut selection }\end{array}$ \\
\hline ADD & 1 & Biomass transpiration coefficient, day ${ }^{-1}$ \\
\hline AGAVE & 2 & Geometric mean of stand age, years \\
\hline AGSUM & 2 & Logarithmic sum of class diameter ages, years \\
\hline $\operatorname{AGTB}(22,60)$ & $1,2,3$ & Minimum age (yrs) of plant(!) with diameter(k) \\
\hline AI1 & $1,2,3,4$ & I1, year number \\
\hline
\end{tabular}

$1=$ Main Program, $2=$ Forman, $3=$ Forcut, $4=$ Rmnant. 
AICHE

AIII

AI1P

AIRCA

AKK

ALPSR

AL9

$\operatorname{AMAC}(16,64)$

2

1,4

1

1

1

1

1

AN

ANMAL $(4,32) \quad 4$

ANOT

AP

AQ

ARIG

ARP

AWATA $(22,16)$

A1(22)

A1TT

A1Z

A2(22)

A22
1,2

2

4

1

1

1

1

1

Input variable, indicates if niche competition will occur, for output purposes only

III, day number

Product of year number $\times 365$

Activity $/ \mathrm{m}^{2}$ in air, for chronic activity function

Solar radiation Ly/day on day $\mathrm{K} 3$

Input variable, lapse rate, ${ }^{\circ} \mathrm{F} / \mathrm{ft}$ rise from reference site

Class diameter index, inches

Input variable, specifies amount of atmospheric activity $/ \mathrm{m}^{3}$ on a given day ( 64 day event possible) for a given year ( 16 separate or continuous years possible)

Class diameter index, inches

Input variable, number of animals grazing during each interval of a given year

Alphanumeric literal used for output purposes only

Facior to determine mortality of certain plant types

I1

Input value, determines if irrigation schedule will be used. For output purposes only

Respiration increment in leaf biomass multiples

Input variable, irrigation water applied, acres/ inches

Activity $/ \mathrm{m}^{2}$ air concentration form all scurces

Weighted average activity/m' over several plant species

Air activity $/ \mathrm{m}^{3}$ due to soil resuspension

Activity $/ \mathrm{m}^{2}$ on leaf surface of specific plant

Activity/gram for leaf biomass due to deposition 


\begin{tabular}{|c|c|c|}
\hline Symbol & Location* & Definition \\
\hline A23 & 1 & $\begin{array}{l}\text { Concentration ratio between deposition activity/ } \\
\text { soil activity }\end{array}$ \\
\hline A55 & 4 & Total number of animals on and off range \\
\hline B & 1 & Mid range monthly temperature, ${ }^{\circ} \mathrm{F}$, for site \\
\hline B & 2 & $\begin{array}{l}\text { Adjusted biomass of stand } \mathrm{g} / \mathrm{m}^{2} \text { to account for } \\
\text { succession and competition }\end{array}$ \\
\hline B & 4 & $\begin{array}{l}\text { Total green and dry biomass, } \mathrm{g} / \mathrm{m}^{2} \text { available for } \\
\text { consumption }\end{array}$ \\
\hline $\begin{array}{l}\text { BAAV } \\
\text { BAP }\end{array}$ & $\begin{array}{l}2 \\
1\end{array}$ & $\begin{array}{l}\text { Logarithmic sum of bole diameters for stand } \\
(0 \text { or } 1) \text { determines whether photosynthesis is } \\
\text { occurring }\end{array}$ \\
\hline $\operatorname{BAR}(60)$ & 2 & Bole diameter, inches \\
\hline $\operatorname{BARSQ}(60)$ & 2 & $\mathrm{BAR}^{2}$, sum of squares of $\log$ bole diameters \\
\hline BATR & 2 & Geometric mean tree height, ft \\
\hline BB & 4 & Fatty acid energy, kcal \\
\hline $\mathrm{BBP}(22)$ & 2 & Previous year (day) above ground biomass $\mathrm{g} / \mathrm{m}^{2}$ \\
\hline $\mathrm{BCP}$ & 1 & $\begin{array}{l}(0 \text { or } 1) \text { determines whether respiration is } \\
\text { occurring }\end{array}$ \\
\hline $\mathrm{BCUT}(22,2)$ & 3 & $\begin{array}{l}\text { Input variable, lower and upper diameters selected } \\
\text { for cutting in "B" class cut selection }\end{array}$ \\
\hline BLANK & 1,2 & Aiphnumeric literal used for output purposes only \\
\hline BMN & 2 & Geometric mean of bole diameter, inches \\
\hline $\mathrm{BP}$ & 1 & Edible portion of plants, $\mathrm{g} / \mathrm{m}^{2}$ \\
\hline BSD & 2 & Geometric standard deviation for basal area \\
\hline BSQ & 2 & Sum of squares of logarithmic basal area \\
\hline $\mathrm{B} 1(22)$ & $1,2,3,4$ & $\mathrm{~g} / \mathrm{m}^{2}$ of live above ground biomass. $1 \leq \mathrm{V}$ \\
\hline $\mathrm{B} 111$ & 1 & $\mathrm{~kg} / \mathrm{m}^{\circ}$, above ground biomass for specific plant \\
\hline B2 & 2 & Diameter of $0-2$ class, inches \\
\hline B5(22) & $1,2,3,4$ & Net daily biomass production, $\mathrm{g} / \mathrm{m}^{2} /$ day \\
\hline $\mathrm{B} 7(22)$ & 1 & $\begin{array}{l}\text { Net daily biomass production shunted to roots, } \\
\mathrm{g} / \mathrm{m}^{2} / \text { day }\end{array}$ \\
\hline
\end{tabular}




\begin{tabular}{|c|c|c|}
\hline Bymbol & Location* & Definition \\
\hline C & 1 & $\begin{array}{l}\text { Amount of water required for photosynthesis, } \\
\mathrm{m} \ell / \mathrm{m}^{2} / \mathrm{g} \mathrm{CH}, \mathrm{O}\end{array}$ \\
\hline $\operatorname{CCUT}(22,2)$ & 3 & $\begin{array}{l}\text { Input variable, lower and upper diameters selected } \\
\text { for cutting in "C" class cut selection }\end{array}$ \\
\hline $\operatorname{CCVV}(22)$ & $1,2,3,4$ & Fractional crown cover for specific plant(k) \\
\hline CFAC(22) & $1,2,3,4$ & Daily structural biomass competition factor, $\mathrm{g} / \mathrm{m}^{\mathrm{a}}$ \\
\hline CHRAC & 1 & $\begin{array}{l}\text { Input variable, initial activity in atmosphere } / \mathrm{m}^{*} \\
\text { for chronic activity function ( } 0.0 \text { or value greater } \\
\text { than } 0.0 \text { required) }\end{array}$ \\
\hline CHRED & 1 & $\begin{array}{l}\text { Input variable, day of final acute contamination } \\
\text { (day number is } 1.0-365.0 \text { or } 0.0 \text { required }\end{array}$ \\
\hline CHREY & 1 & Input variable, year of chronic coutamination \\
\hline CHRSD & 1 & $\begin{array}{l}\text { In put variable, day of initial acute contaminating } \\
\text { event (day number is } 1.0-365.0 \text { or } 0.0 \text { required) }\end{array}$ \\
\hline CHRSY & 1 & $\begin{array}{l}\text { Input variable, year of initial contaminating } \\
\text { chronic event ( } 0.0 \text { or value greater than } 0.0 \\
\text { required) }\end{array}$ \\
\hline CNIT(22) & 4 & Nitrogen content, percentage, of consumed biomass \\
\hline $\operatorname{cov}(60)$ & 2 & $\begin{array}{l}\text { Product of No. of trees } \times \text { diameter class crown cover } \\
\text { for lth diameter class, } m^{\prime}\end{array}$ \\
\hline COVAV & 2 & Sum of crown cover for stand \\
\hline COVSQ(60) & 2 & $\operatorname{COV}(1)^{2}$ sum squares \\
\hline CUTA $(22,8)$ & $1,2,3,4$ & Input variable, harvesting schedules, day of year \\
\hline CVAG & 1 & Total weighted cover of grid due to all plants \\
\hline CVMN & 2 & Mean of crown cover \\
\hline CVMN(22) & 1 & $\begin{array}{l}\text { Crown cover factor used in adjusting total acreage } \\
\text { to grid acreage }\end{array}$ \\
\hline CVSD & 2 & Geometric standard aeviation crown cover for stand \\
\hline CVSQ & 2 & Sum of squares for log crown cover \\
\hline CVTR & 2 & Basal area cover, $\mathrm{m}^{2}$ \\
\hline CVTT & 1 & $\begin{array}{l}\text { Weighted average crown cover of } 2 \text { or more plant } \\
\text { types }\end{array}$ \\
\hline
\end{tabular}




\begin{tabular}{|c|c|c|}
\hline Symbol & Location* & Definition \\
\hline $\operatorname{CSFAC}(22)$ & 1 & $\mathrm{CFAC}(22)$ \\
\hline CYR & 1 & $\begin{array}{l}\text { Input variable, specifies the total number of years } \\
\text { of an acute event ( } 0.0 \text { or value greater than } 0.0 \\
\text { required) }\end{array}$ \\
\hline $\mathrm{C} 1$ & 4 & Rumen capacity, kg \\
\hline $\mathrm{C} 2$ & 4 & Total weight of forage consumed, grams \\
\hline $\mathrm{C} 3$ & 4 & Total activity ingested from forage \\
\hline $\mathrm{C} 4$ & 4 & $\mathrm{~m}^{2}$ grazed by all animal units in a given age group \\
\hline C5 & 4 & Soil ingestion, grams/day \\
\hline $\mathrm{C} 6(22)$ & 2 & Crown diameter to bole diaineter intercept value \\
\hline C6 & 4 & Activity entering blood/day from soil ingestion \\
\hline $\mathrm{C} 7$ & 4 & Fraction air deposition entering blood via GI \\
\hline $\mathrm{C} 7$ & $1,2,3$ & Sum of total volume of all trees for plant(k) \\
\hline C9 & 4 & Activity entering blood from forage (total) \\
\hline $\mathrm{C} 9(22)$ & 2 & Input variable, specific gravity of wood \\
\hline $\mathrm{D}$ & 1 & $\begin{array}{l}\text { Ratio of difference between day no. and Lag period } \\
\text { to day of maximum insolation/ } 365\end{array}$ \\
\hline $\mathrm{D}(22)$ & 4 & $\begin{array}{l}\text { Fraction of diet composed of dry biomass for kth } \\
\text { plant }\end{array}$ \\
\hline DAIC(16) & 1 & $\begin{array}{l}\text { Input variable, determines initial day of acute } \\
\text { event for all years involved }(1.0-365.0)\end{array}$ \\
\hline $\operatorname{DATCT}(22,32)$ & $1,2,3,4$ & $\begin{array}{l}\text { Input variable, stores the years (days) of specific } \\
\text { management generations: cutting, forest fire, etc. }\end{array}$ \\
\hline DD1 & 4 & Digestible fraction of ingested forage \\
\hline DD2 & 4 & Total potential digestibility $\mathrm{kg} / \mathrm{day}$ \\
\hline $\mathrm{DF}$ & 2 & Degrees of freedom for cumulative summations \\
\hline DGTMB & 2 & $\triangle \mathrm{VTMBR}(\mathrm{JK}, 1)$, year $^{-1}$ \\
\hline LIFF & 1 & $\begin{array}{l}\text { Factor which adjusts deepest soil horizon root } \\
\text { fraction }\end{array}$ \\
\hline
\end{tabular}




\begin{tabular}{|c|c|c|}
\hline $\operatorname{DIRA}(22,16)$ & 1 & $\begin{array}{l}\text { Input variable, irrigation schedule, day of year, } \\
\text { when water is applied }\end{array}$ \\
\hline DLY & 1,2 & Alphanumeric literal used for output purposes only \\
\hline DRMM & 1 & Precipitation, $\mathrm{mm}$ \\
\hline DTBR $(22,60)$ & $1,2,3$ & Number of trees/acre for plant(k) with diameter(l) \\
\hline DURC & 1 & $\begin{array}{l}\text { Input variable, duration of an acute event, days } \\
\text { ( } 0.0 \text { or value greater than } 0.0 \text { required) }\end{array}$ \\
\hline D1(22) & $1,3,4$ & $\mathrm{~g} / \mathrm{m}^{2}$ of dead standing biomass for specific plant \\
\hline D3 & 1 & Dead increment/day added to mortality losses $\mathrm{g} / \mathrm{m}^{2} /$ day \\
\hline D3 & 4 & Change in rumen dry matter $\mathrm{kg} /$ day \\
\hline D4 & 4 & Weight of dry forage consumed \\
\hline $\mathbf{E}$ & 1 & $\begin{array}{l}\text { Ratio of difference between day no. and lag period } \\
\text { to day of maximum temperature } 365\end{array}$ \\
\hline $\mathbf{E}$ & 4 & Rumen exit rate, fraction/day \\
\hline EA(22) & 1 & Adjusted evapotranspiration rate, $\mathrm{mm} / 250 \mathrm{~mm}$ \\
\hline EFFP & 1 & End of frost-free period, day number \\
\hline EWAT & 1 & $\mathrm{~g} / \mathrm{m}^{2}$, biomass net production on a given day \\
\hline E1 & 4 & Energy from passed food, kcal \\
\hline E2 & 1 & $\begin{array}{l}\text { Potential evapotranspiration rate, } \mathrm{mm} \text {, from top } \\
250 \mathrm{~mm} \text { soil }\end{array}$ \\
\hline E2 & 4 & Energy from microprotein, kcal \\
\hline E4 & 1 & EA(l) for lth plant series \\
\hline E4 & 4 & Net available energy, kcal \\
\hline E5(22) & 1 & $\begin{array}{l}\text { Effect of temperature }\left({ }^{\circ} \mathrm{C}\right) \text { on photosynthesis for } \\
\text { plant }(l)\end{array}$ \\
\hline E5 & 4 & Fraction of available energy for growth, kcal \\
\hline E6 & 4 & Rate at wlich food passes through GI, fraction \\
\hline E7(22) & 1 & $\begin{array}{l}\text { Effect of soil moisture on photosynthesis for } \\
\text { plant(l) }\end{array}$ \\
\hline
\end{tabular}


F

FDIET

FFP

FRCT

FRMN

FSUPF(4)

F1

F1

F2

F7

$\mathrm{G}(22)$

$\mathrm{GA}(60)$

GA

GGTM

GGTMB

GSUPF

GTDR(3)

GTMBR(22,60)

G1

G1

G3

\section{Ratio of day number/365}

Fraction of diet, specific plant, for animal unit

Input variable, frost-free period of site, days

Input variable, indicates call for Forcut Subroutine, for output purposes only

Input variable, indicates call of Forman Subroutine, for output purposes only

Input variable, fraction of supplementary feed from study area

Normal fermentation rate, fraction of intake/g microprotein/day

Input variable, field capacity of soil, $\% \mathrm{H}_{2} \mathrm{O}$

Fermentation digestion rate, fraction/kg

$\mathrm{mm}$, precipitation as snow

Fraction of diet composed of green biomass for kth plant

Difference in age between youngest and oldest tree in lth class diameter interval

Cumulative green biomass uptake for $k$ th food preference category

Volume for given class diameter (GTMBR)

GTMBR (JK, 1)

Supplementary feed required, grams

Available green standing biomass for ith preference category

Current year virtual volume, $\mathrm{ft}^{\star}$, for plant(l) and diameter(k)

Input variable, elevation of site, feed above sea level

Weight green forage consumed, grams

Growth allocation factor, $\mathrm{ft}^{*}$ 


\begin{tabular}{|c|c|c|}
\hline Bymbol & Location* & Definition \\
\hline G30 & 2 & Growth allocation factor, $\mathrm{ft}^{\circ}$ \\
\hline G4 & 1 & $\begin{array}{l}\text { Random number drawn from a normal distribution } \\
m-0, \sigma=1)\end{array}$ \\
\hline G4 & 2 & $\begin{array}{l}\text { Growth increment, } \mathrm{ft} / \text { year for specific plant(l) } \\
\text { in a given year }\end{array}$ \\
\hline G5 & 1 & $\begin{array}{l}\text { Random number drawn from a normal distribution } \\
(m=0, \sigma=1)\end{array}$ \\
\hline $\mathbf{H}$ & 1 & Input variable, holding capacity of soil, $\% \mathrm{H}_{2} \mathrm{O}$ \\
\hline $\mathbf{H}$ & 4 & Energy in digestible fraction of intake, kcal \\
\hline HD & 4 & Total number of animal uničs on range, maximum \\
\hline HDD & 4 & $\begin{array}{l}\text { Total number of animal units off range (on supple- } \\
\text { mentary feed) }\end{array}$ \\
\hline HERD & 4 & $\begin{array}{l}\text { Number of animal units on range for given grazing } \\
\text { interval }\end{array}$ \\
\hline HF & 1 & Average life-span of plant species \\
\hline HGT(60) & 2 & Height for trees in given diameter class, $\mathrm{ft}$ \\
\hline HGTSQ(60) & 2 & HGT(I) sum of squares \\
\hline HRVSP(4) & 4 & $\begin{array}{l}\text { Previous year biomass, grams, available to kth } \\
\text { animal category as supplementary feed }\end{array}$ \\
\hline HRVST(4) & 4 & $\begin{array}{l}\text { Biomass, grams, available to kth animal category } \\
\text { from all plants utilized as supplementary feed } \\
\text { during current year }\end{array}$ \\
\hline HTAV & 2 & Mean height, feet, for stand \\
\hline HTSD & 2 & Standard deviation for stand height \\
\hline HTSQ & 2 & Sum of squares for log stand height \\
\hline HUMUS(4) & 4 & $\begin{array}{l}\text { Activity/m added to humus compartment from kth } \\
\text { animal category }\end{array}$ \\
\hline $\mathrm{H} 2$ & 4 & Microprotein in rumen, grams \\
\hline H1(22) & 1,4 & $\mathrm{~g} / \mathrm{m}^{2}$ above ground litter-humus \\
\hline $\mathrm{H} 2$ & 1 & Dead biomass temperature decay factor \\
\hline H3 & 1 & Dead biomass soil moisture decay factor \\
\hline
\end{tabular}


I

IA1

IA2

IBRM

IB1

IB2

ICA(22)

ICAAA

ICDY

ICYR

IC1

IC2

IDUR

IICDE

III

IJ

IN

INWRT

INPUTZ

IRG(22)

IRGGG

ISTP
1

1

1

3
Index for months of year

$\operatorname{ACUT}(k, 1)$, portion of stand removed, lower diameter, inches

$\operatorname{ACUT}(k, 2)$, portion of stand removed, upper diameter, inches

TBRM(l), number of tree management events

$\operatorname{BCUT}(k, 1)$, portion of stand removed, lower diameter, inches

BCUT(k,1), portion of stand removed, upper diameter, inches

Day counter for harvesting schedule

ICA(l)

Year counter

Year counter

CCUT(k, 1), portion of stand removed, lower diameter, inches

$\operatorname{CCUT}(\mathbf{k}, 1)$, portion of stand removed, upper diameter, inches

DURC

Defines number of soil horizons in $250 \mathrm{~mm}$ intervals. $1 \leq \mathrm{V} \leq 32$. $(\mathrm{V}=$ value $)$

Day number

Integer value of number of years simulation

Animal category $1 \leq \mathrm{V} \leq 4 .(\mathrm{V}=$ value)

TNWRT(1)

PUTZD(j,k)

Day counter for irrigation schedule

IRG(j)

Determines whether leaf fall occurs at end of frost-free period( 0$)$ or if evergreen(1) 


\begin{tabular}{|c|c|c|}
\hline Symbol & Location* & Definition \\
\hline ITC & 3 & $\operatorname{TCUT}(\mathbf{j}, \mathbf{k})$ \\
\hline $\mathbf{I X X}$ & 4 & ICA(1), see Main Program \\
\hline IYR & 1 & CYR \\
\hline I1 & $1,2,3,4$ & Year counter \\
\hline $\mathbf{I 1 2}$ & 4 & $\begin{array}{l}\text { Switching value for initialization(1) or shunt } \\
(>1)\end{array}$ \\
\hline I7 & 1 & Integer value of RANDN (a random number seed) \\
\hline K3 & 1 & Real value of day number $(1 \leq \mathrm{K} 3 \leq 355)$ \\
\hline L1 & 1 & $\begin{array}{l}\text { Input variable, lag period to maximum insolation, } \\
\text { days }\end{array}$ \\
\hline L1 & 4 & Undigested food $\mathbf{k g} /$ day \\
\hline L2 & 1 & Input variable, lag period to maximum temperature \\
\hline L2 & 4 & Passed undigested food $\mathrm{kg} /$ day \\
\hline L3 & 1 & $\begin{array}{l}\text { Input variable, latitude relative to reference } \\
\text { site }(0.0,1.0,-1.0)\end{array}$ \\
\hline $\mathrm{L} 4$ & 4 & $\begin{array}{l}\text { Fraction of activity from pulmonary deposition } \\
\text { absorbed into blood via stomach/day }\end{array}$ \\
\hline L5 & 4 & $\begin{array}{l}\text { Fraction of activity entering blood from pulmonary } \\
\text { deposition/day }\end{array}$ \\
\hline $\mathbf{M}$ & 1 & Day counter \\
\hline $\mathbf{M}$ & 2 & MIND(l) \\
\hline $\mathbf{M}(8,4)$ & 4 & $\begin{array}{l}\text { Metabolie biomass (kg) for kth population category, } \\
\text { lth animal category }\end{array}$ \\
\hline MIND(22) & 2 & $\begin{array}{l}\text { Year (day) counter for lth plant cycle through } \\
\text { FORMAN }\end{array}$ \\
\hline $\mathbf{M M}$ & 1 & Day counter \\
\hline M1 & 1 & $\begin{array}{l}\text { Maximum photosynthetic efficiency }\left(\mathrm{C}_{5} \text {-plants) }\right. \\
\text { (grams } \mathrm{CH}, \mathrm{O} / \mathrm{m}^{2} \text { ) per (grams green biomass } / \mathrm{m}^{2} \text { ) }\end{array}$ \\
\hline M1 & 4 & Microproh zin passage rate $\mathrm{g} / \mathrm{g} / \mathrm{day}$ \\
\hline M2 & 4 & Grams/kcal of micruprotein in digestible fraction \\
\hline
\end{tabular}


M4

M5

M6

M7

$\mathrm{N}$

NDAY(12)

$\mathrm{NN}$

NRE

N2

N3

N4

N5(4)

o

ONRNG $(4,32) \quad 4$

$\mathbf{P}$

$P(8,4)$

PI

PLNTS

PNIT(22)

PP6(22)

PRCV

PSE
4

1

4

1

1

4

4

4

1

1

1

4

4

4

4

4

4

4

1

1
Definition

Microprotein/metabolic biomass ration g/kg

$\mathrm{m}^{2}$ grazed by an age group of a given animal population

Fraction of area grazed by an age group of a given animal population

Area of region being grazed, $\mathrm{m}^{2}$, by animal category (grid area)

Nitrogen content of ingested forage, \%

Number of days in each month

Integer value of $\mathrm{Q}(\mathrm{l})$ or integer value of $\mathrm{Q}(\mathrm{l})+1$

TRE

Nitrogen content of daily photosynthate, \%

Nitrogen content of dry forage, $\%$

Nitrogen content of standing green forage

Input variable, number of animal units/category

Ingestion rate, $\mathrm{kg} / \mathrm{day}$

Input variable, days on range for year intervals (100-290), (150-300), etc.

Daily precipitation events

Microprotein level, grams, in rumen for kth age, lth animal

Constant, 3.1416

Number of plant types in simulation

Nitrogen content, percentage, of photosynthate for lth plant category

Initial leaf biomass for 22 plant categories

Input variable, coefficient of variation for precipitation/event of reference site

PSEP 


\begin{tabular}{|c|c|c|}
\hline Eymbol & Location" & Definition \\
\hline PSEP & 1 & $\begin{array}{l}\text { Distinguishes } C_{5} \text {-plant(1) or } C_{4} \text {-plant }(0.5), \\
\text { photosynthetic efficiency }\end{array}$ \\
\hline PUTZD(4,22) & $1,2,3,4$ & Input variable, plants utilized by animal(l) \\
\hline P1 & 1 & Annual cumulative precipitation, $\mathrm{mm}$ \\
\hline $\mathbf{P 2}$ & 4 & Needle or leaf biomass $\mathrm{g} / \mathrm{m}^{2}$ (green) \\
\hline P3 & 4 & Needle or leaf biomass $\mathrm{g} / \mathrm{m}^{2}$ (dry standing) \\
\hline P4(22) & 4 & Growth rate fraction/day for kth plant category \\
\hline P5(4) & 4 & $\begin{array}{l}\text { Input variable, fraction of muscle tissue and } \\
\text { organs consumed in study area in that region }\end{array}$ \\
\hline $\mathbf{Q}(24)$ & 1 & $\begin{array}{l}\text { Product of random number (0-1) and number of days } \\
\text { in given month }\end{array}$ \\
\hline $\mathbf{Q}(8,4)$ & 4 & $\begin{array}{l}\text { Accumulated activity entering pulmonary region for } \\
\text { kth plant, lth animal }\end{array}$ \\
\hline QDO & 4 & $\begin{array}{l}\mathrm{g} / \mathrm{m}^{2} \text { of dry forage consumed by animal units of } \\
\text { given age group/day }\end{array}$ \\
\hline QFRAC(3) & 4 & $\begin{array}{l}\text { Animal preference fraction for kth food preference } \\
\text { category }\end{array}$ \\
\hline QGO & 4 & $\begin{array}{l}\mathrm{g} / \mathrm{m}^{2} \text { of green forage consumed by animal units of } \\
\text { given age group/day }\end{array}$ \\
\hline QSUM & 4 & $\begin{array}{l}\text { Available forage } \mathrm{g} / \mathrm{m}^{2} \text { from all forage sources of } \\
\text { ith preference category }\end{array}$ \\
\hline Q1 & 1 & $\begin{array}{l}\text { Factor used in calculating effect of soil moisture } \\
\text { on photosynthesis }\end{array}$ \\
\hline Q8 & 1 & Random number counter \\
\hline Q10 & 4 & $\begin{array}{l}\text { Available forage for consumption on a daily basis } \\
\mathrm{g} / \mathrm{m}^{\mathbf{s}} \text { for specific plants utilized }\end{array}$ \\
\hline $\mathbf{R}$ & 1 & Input variable, annual precipitations, inches \\
\hline $\mathbf{R}(\mathbf{8 , 4 )}$ & 4 & $\begin{array}{l}\text { Rumen capacity, kg, for a given age }(\mathrm{yr}) \text { category of } \\
\text { a specific animal category }\end{array}$ \\
\hline RACUT(22) & 3 & Input variable, fraction of ACUT cut left standing \\
\hline RANDN & 1 & Input variable, random number seed for a given run \\
\hline
\end{tabular}




\begin{tabular}{|c|c|c|}
\hline Symbol & Location* & Defnition \\
\hline RANK(22) & 1 & $\begin{array}{l}\text { Succession ranking according to dominance }(1 \leq \mathrm{V} \\
\leq 22) \text { for plant. }(\mathrm{V}=\text { value })\end{array}$ \\
\hline RAP & 1 & Constant in plant respiration equation (gate) \\
\hline RBCUT(22) & 3 & Input variable, fraction of BCUT cut left standing \\
\hline RCCUT(22) & 3 & Input variable, fraction of RCUT cut left standing \\
\hline RCODE(22) & 1 & $\begin{array}{l}\text { Input variable, determines soil depth for specific } \\
\text { plant type, mm }\end{array}$ \\
\hline $\mathrm{RD1}(22)$ & $1,2,3,4$ & $\mathrm{~g} / \mathrm{m}^{2}$ dead root biomass \\
\hline RD3 & 1 & Daily mortality increment from roots $\mathrm{g} / \mathrm{m}^{2} /$ day \\
\hline REEL & 1 & Number of soil horizons at $250 \mathrm{~mm}$ increments $\times 10$ \\
\hline RELV & 1 & Input variable, elevation, feet above sea level \\
\hline RFC & 1 & $\begin{array}{l}\text { Reciprocal of soil evapotranspiration factor at } \\
\text { given soil depth }\end{array}$ \\
\hline RFP & 1 & Constent in plant respiration equation (gate) \\
\hline RGAIN & 1 & $\begin{array}{l}\text { Activity/ms absorbed into roots in a specific soil } \\
\text { horizon }\end{array}$ \\
\hline RGC(22) & $1,2,3,4$ & $\begin{array}{l}\text { Input variable, selector for specific plants to be } \\
\text { simulated }\end{array}$ \\
\hline RHERB & 1 & $\begin{array}{l}\text { Input variable, determines whether Rmnant Sub- } \\
\text { routine is called } g / \mathrm{m}^{2} \text { root litter-humus }\end{array}$ \\
\hline RH1(22) & 1 & $\mathrm{~g} / \mathrm{m}^{\mathbf{3}}$ root litter-humus \\
\hline RINGT(22) & $1,2,3,4$ & Diameter increment/tree of given diameter \\
\hline RIRRC(22) & 1 & $\begin{array}{l}\text { Input variable, determines whether irrigation } \\
\text { schedule for plant type will be part of input }\end{array}$ \\
\hline RNDD & 1 & $\begin{array}{l}\text { Integer value of number of soil horizons at } 250 \mathrm{~mm} \\
\times 10\end{array}$ \\
\hline RPNTP & 1 & $\begin{array}{l}\text { Root penetration, mm, as a function of total days } \\
\text { elapsed }\end{array}$ \\
\hline $\mathbf{R R}(\mathbf{4})$ & 4 & $\begin{array}{l}\text { Input variable, indicates which of four animal types } \\
\text { are simulated. Value }=\text { mature weight ratio of animal } \\
\text { unit to mature weight of beef cow }\end{array}$ \\
\hline
\end{tabular}




\begin{tabular}{|c|c|c|}
\hline RSBIO(22) & 1 & $\begin{array}{l}\mathrm{g} / \mathrm{m}^{3} / \text { day maintenance losses exclusive of leaf } \\
\text { biomass }\end{array}$ \\
\hline RSCDE & 1 & $\begin{array}{l}\text { Input variable, determines whether succession } \\
\text { module is used(1) or ignored(0) }\end{array}$ \\
\hline RSCF(22) & $1,2,3,4$ & Effective life-span for succession, years \\
\hline RSS(22) & 1 & $\begin{array}{l}\text { Input variable, determines which plant groups are } \\
\text { to be considered for competition for available land } \\
\text { area }\end{array}$ \\
\hline RSUSP & 1 & Resuspension factor, day ${ }^{-1}$ \\
\hline RTRANS & 1 & Fraction of photosynthate translocated to roots \\
\hline RVJ & 3 & $\begin{array}{l}\text { "Reverse-j" cut equation, fraction for given } \\
\text { diameter class }\end{array}$ \\
\hline RXPP & 1 & Constant in plant respiration equation \\
\hline R1(22) & $1,2,3,4$ & $\mathrm{~g} / \mathrm{m}^{2}$ live root biomass \\
\hline R111 & 1 & $\mathrm{~g} / \mathrm{m}^{2}$ live root biomass \\
\hline $\mathbf{S}(\mathbf{8 , 4 )}$ & 4 & $\begin{array}{l}\text { Rumen fill, kg, for kith population age group of lth } \\
\text { animal category }\end{array}$ \\
\hline SAAP(4) & 4 & SPAA(4) from previous year \\
\hline SACT(3) & 4 & $\begin{array}{l}\text { Specific activity of ith preference category, } \\
\text { activity/g }\end{array}$ \\
\hline SAFP & 1 & $\begin{array}{l}\text { Constant used in determining effect of soil } \\
\text { moisture on photosynthesis }\end{array}$ \\
\hline SAIR(3) & 4 & $\begin{array}{l}\text { Specific activity, activity/g, for kth food } \\
\text { preference category (surface contamination) }\end{array}$ \\
\hline SAO & 4 & $\begin{array}{l}\text { Specific activity, activity/g, derived from surface } \\
\text { contamination }\end{array}$ \\
\hline $\operatorname{SATB}(22,60)$ & $1,2,3$ & $\begin{array}{l}\text { Specific activity, activity/g, for plant(k) of } \\
\text { diameter(l) }\end{array}$ \\
\hline SCTP(22) & $1,2,3,4$ & Distinguishes woody plants(0) from herb types(1) \\
\hline SDPTH & 1 & $\begin{array}{l}\text { Input variable, soil depth, mm (equals } 0.0 \text { if } \\
\text { input via other card input, see } R C O D E \text { ) for site }\end{array}$ \\
\hline SFAC(22) & $1,2,3,4$ & Succession factor calculated on yearly basis \\
\hline
\end{tabular}




\begin{tabular}{|c|c|c|}
\hline Symbol & Location* & Dofinition \\
\hline SFCT & 1 & $\begin{array}{l}\text { Sum of succession factor SFAC(22) for specific } \\
\text { plants (intermediate sum 1) }\end{array}$ \\
\hline SFFA & 1 & $\begin{array}{l}\text { Sum of succession factor SFAC(22) for specific } \\
\text { plants (intermediate sum 2) }\end{array}$ \\
\hline SFFB & 1 & $\begin{array}{l}\text { Sum of succession factor SFAC(22) for specific } \\
\text { plants (intermediate sum 3) }\end{array}$ \\
\hline SFFC & 1 & $\begin{array}{l}\text { Sum of succession factor SFAC(22) for specific } \\
\text { plants (final summation) }\end{array}$ \\
\hline SFFP & 1 & Beginning of frost-free period, day number \\
\hline SNIT(22) & 4 & $\begin{array}{l}\text { Nitrogen content, percentage, of standing green } \\
\text { biomass }\end{array}$ \\
\hline SNM & 1 & Snow melt, as mm water \\
\hline SOILF(22) & 1 & Soil evapotranspiration adjustment for soil depth \\
\hline SOILP & 1 & Reciprocal of SOILF \\
\hline SP & 1 & $\begin{array}{l}(0,1) \text { controls uptake of activity below a given } \\
\text { depth of soil }\end{array}$ \\
\hline SPAA(4) & 4 & $\begin{array}{l}\text { Total surface activity available to kth animal. } \\
\text { category for irrigated forage }\end{array}$ \\
\hline SPAC(4) & 4 & $\begin{array}{l}\text { Specific activity, activity/g, for given category } \\
\text { consumed daily by kth animal category }\end{array}$ \\
\hline SPAP(4) & 4 & SPAS(4) from previous year \\
\hline SPAR(4) & 4 & $\begin{array}{l}\text { Specific activity, activity/g, on surface for given } \\
\text { food category consumed by kth animal category }\end{array}$ \\
\hline SPAS(4) & 4 & $\begin{array}{l}\text { Total activity available to kth animal category } \\
\text { from irrigated forage }\end{array}$ \\
\hline SPUT(4) & 4 & $\begin{array}{l}\text { Total sum of plants utilized for kth animal } \\
\text { category }\end{array}$ \\
\hline STBMX & 1 & Maximum value of STBR(k) \\
\hline STBR(22) & 1 & $\begin{array}{l}\text { Structural biomass succession factor, } \mathrm{g} / \mathrm{m}^{2} / \mathrm{yr} \\
\text { running yearly average }\end{array}$ \\
\hline STRANS & 1 & $\begin{array}{l}\text { Fraction of photosynthate translocated to above } \\
\text { ground biomass }\end{array}$ \\
\hline SYAV & 1 & Cumulative sum of yearly interations, years \\
\hline
\end{tabular}




\begin{tabular}{|c|c|c|}
\hline Symbol & Location" & Definition \\
\hline $\mathbf{S} 1$ & 1 & Current value of soil moisture availability \\
\hline S10 & 4 & $\begin{array}{l}\text { Specific activity of consumed forage for internal } \\
\text { absorption }\end{array}$ \\
\hline $\mathbf{T}$ & 1 & Temperature ${ }^{\circ} \mathrm{C}$ on day $\mathrm{K} 3$ \\
\hline TAV & 1 & Average annual temperature, ${ }^{\circ} \mathbf{F}$ \\
\hline TBRM(22) & $1,2,3,4$ & $\begin{array}{l}\text { Input variable, indicates how many managed cuttings } \\
\text { or events will be simulated }\end{array}$ \\
\hline TCR & 2 & $\begin{array}{l}\text { Determines whether plant(l) is cycling on yearly(1) } \\
\text { or daily basis ( } 365 \text { ) in Forman }\end{array}$ \\
\hline TCUT $(22,32)$ & 3 & $\begin{array}{l}\text { Input variable, specifies type of cut or fire event } \\
\text { or event parameter }\end{array}$ \\
\hline TLAl(22) & 1 & Leaf area index $\mathrm{m}^{2} / \mathrm{m}^{\prime}$ for specific plant category \\
\hline TMXV & 1 & $\begin{array}{l}\text { Input variable, maximum temperature variation, }{ }^{\circ} \mathrm{F} \text {, } \\
\text { on a monthly basis }\end{array}$ \\
\hline TNIT(3) & 4 & $\begin{array}{l}\text { Sum of total nitrogen concentration } \times 100 \text { for ith } \\
\text { preference group }\end{array}$ \\
\hline TNWRT(22) & 2 & $\begin{array}{l}\text { Input variable, indicates how many print-outs of } \\
\text { tree structure are required. Maximum is } 20\end{array}$ \\
\hline TOTAC & 2 & $\begin{array}{l}\text { Total activity, activity/g, of total stand, } \\
\text { cumulative }\end{array}$ \\
\hline TOTSA & 2 & Above ground biomass for stand activity/m \\
\hline TOTSF(4) & 4 & $\begin{array}{l}\text { Total supplementary feed required by kth animal } \\
\text { category on a given day }\end{array}$ \\
\hline TPGNW(22) & $1,2,3,4$ & Running average productivity $\mathrm{kg} / \mathrm{m}^{\mathrm{L}} / \mathrm{year}$ \\
\hline TRE & 1 & Total rainfall events/month totaling more than $0.1^{\prime \prime}$ \\
\hline TREES & 2 & Total number of trees in stand \\
\hline TSUMB & 4 & $\mathrm{~g} / \mathrm{m}^{2}$ of green and dry standing biomass \\
\hline TY & 1 & Year counter \\
\hline $\mathrm{T} 1$ & 1 & $\begin{array}{l}\text { Input variable, minimum solar radiation, } L y / \text { day } \\
\text { receivad during the year }\end{array}$ \\
\hline
\end{tabular}




\begin{tabular}{|c|c|c|}
\hline Symbol & Location* & Definition \\
\hline T1 & 2 & $\begin{array}{l}\text { Minimum volume for given biomass density required } \\
\text { for Forman access }\end{array}$ \\
\hline T111 & 1 & Total respiration leaf and maintenance, $\mathrm{g} / \mathrm{m}^{\mathrm{a}} / \mathrm{day}$ \\
\hline T2 & 1 & $\begin{array}{l}\text { Input variable, maximum solar radiation, Ly/day } \\
\text { received during the year }\end{array}$ \\
\hline T3 & 1 & $\begin{array}{l}\text { Input variable, mimimum monthly temperature, }{ }^{\circ} \mathrm{F} \text {, } \\
\text { average for site on a daily basis }\end{array}$ \\
\hline T4 & 1 & $\begin{array}{l}\text { In put variable, maximum monthly temperature, }{ }^{\circ} \mathrm{F} \\
\text { average for site on a daily basis }\end{array}$ \\
\hline T5 & 1 & $\mathrm{~m} \ell / \mathrm{m}^{2}$ water evapotranspired/day \\
\hline T5P(22) & 1 & $\mathrm{~m} \ell / \mathrm{m}^{2}$ water evapotranspired for a specific plant/day \\
\hline T6 & 1 & $\begin{array}{l}\text { Potential amount of water required for photosyn- } \\
\text { thesis } \mathrm{m} l / \mathrm{m}^{2} / \text { day }\end{array}$ \\
\hline T8 & 1 & Cumulative day counter \\
\hline $\mathrm{U}(8,4)$ & 4 & $\begin{array}{l}\text { Digestible dry matter, } \mathrm{kg} / \text { day, intake rate for kth } \\
\text { population group of lth animal category }\end{array}$ \\
\hline UP & 4 & Uptake kg/day for ith preference categories \\
\hline UPTKE & 1 & $\begin{array}{l}\text { Input variable, uptake coefficient, fraction } / \text { day } / \mathrm{m} \ell \\
\text { transpiration } / \mathrm{m}^{\mathbf{3}}\end{array}$ \\
\hline $\mathrm{V}(8,4)$ & 4 & $\begin{array}{l}\text { Activity entering organism from all sources for kth } \\
\text { animal categories }\end{array}$ \\
\hline $\mathrm{V}(1, \mathrm{I})$ & 4 & Activity in new born animal units \\
\hline $\operatorname{VA}(60)$ & 2 & $\begin{array}{l}\text { Activity } / \mathrm{m}^{2} \text { of lth class diameter for specific } \\
\text { plants }\end{array}$ \\
\hline VDTBM & 2 & $\triangle \mathrm{VTMBR}(\mathrm{JK}, 1)$ for succeeding years \\
\hline VCUT(60) & 3 & $\begin{array}{l}\text { Residual volume of timber, } \mathrm{ft}^{\prime} \text {, after harvesting } \\
\text { operation or fire }\end{array}$ \\
\hline VOLTR & 2 & Volume of tree, "ft"," of given diameter \\
\hline VTMBR(22,60) & $1,2,3,4$ & Volume, cubic feet, of plant(l) for diameter(k) \\
\hline VTOT & 3 & $\begin{array}{l}\text { Total volume of timber harvested for a specific } \\
\text { type of cut }\end{array}$ \\
\hline
\end{tabular}




\begin{tabular}{|c|c|c|}
\hline Symbol & Location* & Definition \\
\hline VVTM & 2 & Current biomass volume, cubic feet \\
\hline V2 & 2 & Volume of $0-2$ inch diameter class plant, $\mathrm{ft}^{\mathrm{t}}$ \\
\hline V3 & 2 & Volume of 2-4 inch diameter class plant, $\mathrm{ft}^{\mathrm{t}}$ \\
\hline Vs & 2 & Wood of stand, $\mathrm{ft}^{\prime}$ \\
\hline V4M6 & 2 & $\begin{array}{l}\text { Volume difference stand generated volume and cal- } \\
\text { culated volume }\end{array}$ \\
\hline V5 & 2 & $\begin{array}{l}\text { Virtual stand volume, } \mathrm{ft}^{\mathrm{s}} \text {, required for setting } \\
\text { radial growth rate of stand boles }\end{array}$ \\
\hline V6 & 2 & Sum of volumes, $\mathrm{ft}^{2}$, for all class diameters \\
\hline V8(22) & $1,2,3,4$ & $\begin{array}{l}\text { Taper coefficient relating stem diameter to above } \\
\text { ground biomass and specific gravity of wood (final value). } \\
\text { Regression coefficient - } 1 \text { bole diameter to biomass density } \\
\text { (initial value) }\end{array}$ \\
\hline $\mathbf{W}(8,4)$ & 4 & Biomass, $\mathrm{kg}$, for 4 animal classes, 8 age groups \\
\hline$W(9,4)$ & 4 & Total activity/animal unit 3-8 year oids for 4 animal classes \\
\hline $\mathbf{W}(\mathbf{1 0 , 4 )}$ & 4 & Average activity/animal unit for total herd \\
\hline $\mathbf{W}(11,4)$ & 4 & Concentration in milk, activity, liter \\
\hline$W(12,4)$ & 4 & Average weight/animal unit for total herd \\
\hline $\mathbf{W}(13,4)$ & 4 & Activity/kg in muscle tissue of herd (weighted ave) \\
\hline$W(14,4)$ & 4 & Activity/kg in liver (weighted ave) \\
\hline$W(15,4)$ & 4 & $\begin{array}{l}\text { kg of muscle available to human population from } \\
\text { herd }\end{array}$ \\
\hline$W(16,4)$ & 4 & Activity $/ \mathrm{kg}$ in bone \\
\hline WA(22) & 1 & $\begin{array}{l}\text { Amount of water, mm, in top } 250 \mathrm{~mm} \text { adjusted for soil } \\
\text { depth }^{2} \mathrm{~W} 1 \leq \mathrm{V} \leq \mathrm{H} \text {. (V = value) }\end{array}$ \\
\hline WATER(22) & 4 & Water content, percentage, of consumed biomass \\
\hline WND(22) & $1,2,3,4$ & Leaf biomasss, $\mathrm{g} / \mathrm{m}^{2}$, for a specific plant \\
\hline WN1 & 1 & Needle or leaf biomass $\mathrm{kg} / \mathrm{m}^{2}$ \\
\hline WRSTR(22,20) & 2 & $\begin{array}{l}\text { Input variable, stores the years (days) of } \\
\text { specific output }\end{array}$ \\
\hline
\end{tabular}




\begin{tabular}{|c|c|c|}
\hline Symbol & Lacation* & Definition \\
\hline WURAT(22) & 1 & Annual net production $\mathrm{g} / \mathrm{m}^{2} /$ year for specific plant \\
\hline WUTP & 1 & $\begin{array}{l}\text { Maximum yearly water utilization efficiency for } \\
\text { photosynthesis } \mathrm{m} / \mathrm{g} \mathrm{CH}, \mathrm{O}\end{array}$ \\
\hline WUYAW(22) & 1 & Net yearly productivity $\mathrm{g} / \mathrm{m}^{2} /$ year \\
\hline WYAV(22) & 1 & $\begin{array}{l}\text { Cumulative net yearly productivity } g / \mathrm{m}^{2} / \text { year by } \\
\text { specific plant }\end{array}$ \\
\hline W1 & 1 & Input variable, wilting point of soil, $\% \mathrm{H}_{2} \mathrm{O}$ \\
\hline W11 & 1 & Potential leaf biomass, $\mathrm{kg} / \mathrm{m}^{2}$ \\
\hline W3 & 4 & Animal weights, mature weight ratio \\
\hline W5 & 4 & Weight gain, kg \\
\hline $\mathrm{X}(12)$ & 1 & $\begin{array}{l}\text { Input variable, January through December monthly } \\
\text { precipitation }\end{array}$ \\
\hline$X(60)$ & 2 & $\begin{array}{l}\text { Total advantage factor for lth diameter class for } \\
\text { biomass allocation }\end{array}$ \\
\hline$X(8,4)$ & 4 & $\begin{array}{l}\text { Blood weight(kg) for kth population category; lth } \\
\text { anima! category }\end{array}$ \\
\hline XHERB & 1,4 & $\begin{array}{l}\text { Input variable, controls output in RMNANT Sub- } \\
\text { routine on a daily basis. The value indicates the } \\
\text { years when such output will occur (modulus) }\end{array}$ \\
\hline $\mathrm{XJ} i(22)$ & 2 & $\begin{array}{l}\text { Input variable, size of diameter class intervals } \\
\left(--- \text { etc. } 2^{\prime \prime}-0.2^{\prime)}\right.\end{array}$ \\
\hline XPLNT & 1 & $\begin{array}{l}\text { Input variable, year for daily output of plant } \\
\text { simulation }\end{array}$ \\
\hline XWRT & 2 & $\begin{array}{l}\text { Switching constant for specific activity deter- } \\
\text { mination }\end{array}$ \\
\hline $\mathbf{X} 1$ & 4 & Per cent of fermented energy as fatty acids \\
\hline $\mathrm{X} 8(22)$ & 1 & $\begin{array}{l}\text { Ratio of root specific activity to top } 1 \text { " soil } \\
\text { specific activity }\end{array}$ \\
\hline $\mathbf{Y}$ & 1 & Random number \\
\hline $\mathrm{Y}(8,4)$ & 4 & $\begin{array}{l}\text { Inhalation rate } \mathrm{m}^{2} / \text { day for kth population category, } \\
\text { lth animal category }\end{array}$ \\
\hline
\end{tabular}




\begin{tabular}{|c|c|c|}
\hline Symbol & Location* & Definition \\
\hline YRCNT(16) & 1 & $\begin{array}{l}\text { Input variable, determines specific years for } \\
\text { application of an acute event. The year may be } \\
\text { sequential or nonsequential but equal to CYR }\end{array}$ \\
\hline YRGRZ $(4,32)$ & $1,2,3,4$ & $\begin{array}{l}\text { Input variable, varied year grazing intervals } \\
(5-50),(60-80) \text {, etc. }\end{array}$ \\
\hline YRLY & 1,2 & Alphanumeric literal used for output purposes only \\
\hline Y1 & 1 & $\begin{array}{l}\text { Input variable, activity of soil, activity/g dry } \\
\text { weight in soil }\end{array}$ \\
\hline Y2 & 1 & $\begin{array}{l}\text { Input variable, allocates a specific year to obtain } \\
\text { daily output }\end{array}$ \\
\hline $\mathrm{Z}(60)$ & 2 & Volume of average iree, $\mathrm{ft}^{\prime}$, in class diameter(l) \\
\hline$Z(8,4)$ & 4 & $\begin{array}{l}\text { Population structure, fraction of total herd for kth } \\
\text { population category; lth animal category }\end{array}$ \\
\hline $\mathrm{ZF}(22,32)$ & 1 & $\begin{array}{l}\text { Input variable, root fraction in given soil } \\
\text { horizon }\end{array}$ \\
\hline ZFLL & 1 & RFC \\
\hline $\mathbf{Z P}$ & 1 & Activity/gram in soil, top $250 \mathrm{~mm}$ horizon \\
\hline $\mathrm{ZU}(22,32)$ & 1 & $\begin{array}{l}\text { Input variable, uptake coefficient for a radio- } \\
\text { nuclide in a given soil horizon }\end{array}$ \\
\hline $\mathrm{Z} 1(2,32)$ & $1,2,3,4$ & $\begin{array}{l}\text { Input variable, soil activity, activity, gram dry } \\
\text { weight }\end{array}$ \\
\hline $\mathrm{Z} 2(22)$ & $1,2,3$ & Activity $/ \mathrm{m}^{2}$ in live root of specific plant \\
\hline $\mathrm{Z3}(22)$ & $1,2,3,4$ & $\begin{array}{l}\text { Activity } / \mathrm{m}^{2} \text { in live above ground biomass of } \\
\text { specific plant }\end{array}$ \\
\hline $\mathrm{Z4}(22)$ & $1,2,3,4$ & Activity $/ \mathrm{m}^{2}$ in dead standing above ground biomass \\
\hline $\mathrm{Z} 4 \mathrm{R}(22)$ & $1,2,3,4$ & Activity $/ \mathrm{m}^{2}$ in dead standing root biomass \\
\hline $\mathbf{Z 5}(22)$ & $1,2,3,4$ & Activity $/ \mathrm{m}^{\mathbf{1}}$ in litter-humus produced above ground \\
\hline $\mathrm{Z5R}(22)$ & 1 & Activity $/ m^{2}$ in litter-humus produced below ground \\
\hline $\mathrm{Z6}(22)$ & 1 & Activity/g in live root compartment \\
\hline $\mathrm{Z} 7(22)$ & 1 & Activity/g in live above ground biomass \\
\hline Z7P & 1 & Activity/g in dead standing above ground biomass \\
\hline Z7PR & 1 & Activity/g in dead standing root biomass \\
\hline
\end{tabular}




\section{Bymbol}

Z77

$\mathbf{Z} 8(22)$

Z9(22)

Z9R(22)

Z99

\section{Definition}

Ratio of surface and internal specific activity to top 1" specific act:vity of soil

Ratio of above ground specific activity to top 1" specific activity of soil

Cumulative activity $/ \mathrm{m}^{2}$ in litter-humus produced above ground

Cumulative activity $/ \mathrm{m}^{2}$ in litter-humus produced below ground

Call statement, see Z9(1) in Main Program 


\section{LASL Identification}

No. LP-711 BIOTRAN

PROCRAM PLANTS (INPUT.OUTPUT)

COMNON HI (22), CCWV(22), Al (22), A2(22), BI (22), RI (2E), Ol (22),

PLA

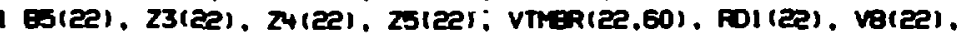

PLA

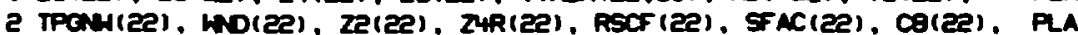

$321(22,321$. CFAC(22)

PLA

COMHON MCCRZ(4,32), PUTZD(4,22), ROC(22), ICA(22), ACRES(22), OUTA PLA

$1(22,8)$

PLA

COMMON DTER(22,60). AGTB(22,60), MTB(22,60), SATB(22,60), CB(22) PLA COMTON GTMER(22,60). DATCT(22.32), TEFM(22), SCTP(22), RINGT(22) PLA

Dimension $x(12)$, Q $(24)$. NN(24), NDAY(12), EA(z2), RIRAC(22)

DIMENSION Es(22), E7(22), MAl22), AMATA(22,16), DIRA(22,16)

DIMEnsian ZU(22,32). ZF(22.32), Mu(22), B7(22), FHI(22), Z6(22)

DIMENSION $27(22), 28(22)$, 29(22), ZSR(22), XB(22)

DIMENSION IRG(22), HYAVI2EI, RURAT(22), CXFAC(22)

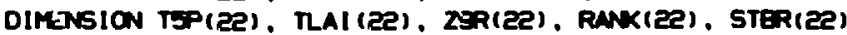

DIMENSION RSS(22), CVMX(22), RSBIO(22), RCOOE (22), SOILF(22)

DIMENSION VRCNT (16), DAIC(16), AMC (16,64),RSUSP(22)

FEA MI, K2, K3, LI, L2, K4, L3

DATA (NDAY(1), I=1,12) /30.27,30.29,30,29.2*30,29,30.29.30/

DATA MNT, RANK, OLY, YRLY /3ANT,3H , GDAILY, GMEARYY

10 FEAO 1570, TI,T2,LI,T3, T4,L2

IF (TI.EO.0.0) CALL EXIT

READ 1570, TFE, MORV, FACV, RELV, NLPSR

NPE=TRE

READ 1570, $(x(1), 1=1,12)$

READ 1570, R,G1,L3,FI, W1 ,H, Y1,Ye

READ 1570. RSCDE, FAERB, RANON, FREN, ARIG, AICE, FRCT

IRIG=ARIG

NICHE=AICEE

READ 1570. (AOC (L) ,L-1, 22)

READ 1570, (RIRRC(L), L-1,22)

READ 1570. (RSS (L) ,L-1.22)

PEAD 1570. (ACRES(L), L=1,22)

READ 1570, (RCOOE (L), L=1,22)

READ 1570, CHRAC,CYR,DUR, CHPSY, CHEY, CAPSO, CARED

IYR-CYR

IDUR-DURC

IF (IYR.EO.D) CO TO 30

READ 1570. (YRCNT (K), $K=1$, IYR)

READ 1570, (DAIC (K), K=1, IYR)

DO 20 L-1, IYR

FEND 1570, (AMAC (L, K), K=1, 10UR)

20 CONTINLE

30 CONTINEE

$F 1=1 \cdot 2.50$

$\mathrm{WI}+\mathrm{HI} \cdot 2.50$

$H+H \cdot 2.50$

PLNTS $=0.0$

DO $50 \mathrm{~K}=1$, 22

$A K=K$

IF (ROC(K). GT.0.0) PLNTS=PLNTS+1.0

Wh $(K)=W 1$

$\operatorname{RINGT}(K)=0.0$

$E 5(K)=0.0$

$E 7(K)=0.0$

$E A(K)=0.0$

$\operatorname{RANK}(K)=0.0$

$\operatorname{TEPM}(K)=0.1$

DO $50 \mathrm{~J}=1.16$

AWATA $(K . J)=0.0$

IF (J.GT.8) 60 TO 40

$\operatorname{CUTA}(K, J)=0.0$

$\operatorname{DIRA}(K, J)=0.0$

40 CONTINUE 
50 CONT IME

READ 1570, FFP, SDPTH,UPTK, XPLNT, XERA

UPTELUPTEE- . OE-OS

$0090 \mathrm{~J}=1,22$

SOIUF $(J)=0.0$

If (RKC)(J).EO.0.0) 00 To 90

SOILF (J)=0.632/ (1.0-EXP(-.004.SDPTH) )

If (nCODE(J).EO.0.0) CO TO 60

SOLLF $(J)=0.632 /(1.0-E X P(-.004 * R C O D E(J))$

I ICDE-INT (RCODE (J)/250.0)+1 .0

READ 1560, (Z) (J,L),L=1,11CDE)

READ 1550, (ZF $(J, L), L=1,1$ (CDE)

REAO 1560, (ZU) J,LI,L=1, I ICDE)

CO TO 80

60 CONTINEE

RCOCE $(J)=1 N T(S O P T H / 250)+$.

I (COE-RCOOE $(J)+1.0$

RCODE $(J)=P C O D E(J) \cdot 250.0$

ZF $(J, 1)=0.0$

$Z U(J, 1)=0.0$

$Z 1(J, 1)=Y)$

FrC $=0.0$

Do 70 LE2, 1 ICOE

NLL-1

$Z !(J, L)=$ UPTKE

Zi $(J, L)=Y i$

सRU $=.632 /(1 .-E X P(-.004=250 . \cdot N L))$

즌 $=1.0 / 2$ 니

SOILP=1.0/SOILF (L)

IF (ZLL.GE.SOILP.AND. I ICOE.EO.E) ZZL=SOILP

ZF $(J, L)=(Z F L L F F C) / S O I L P$

FFC-2ㄴㄴ

70 contine

FNDO=10* INT CSOPTH/ZEO . )

REZI $=10.0 * 5 D P T H / E S O$.

DIFF $=($ REE - FNDO $) / 10.0$

ZU(J.11COE $)=Z(J, 1$ ICDE $) \cdot 0 I F F$

BO CONTINE

go CONTINE

$F 7=0.0$

$51=41$

$P 1=3.1416$

DO 100 L-1.2P

MAAL) $=0.0$

$\operatorname{CCW}(L)=0.0$

$A P(L)=0.0$

DI (L) $=1$.

$H I(L)=1$.

$\theta 1(L)=1$.

$R \mid(L)=1$.

$R D \mid(L)=1$.

PHI (L) $=1$.

$A \mid(L)=0$.

$E S(L)=0$.

$\theta 7(L)=0$.

$22(L)=0$.

$23(L)=0$.

$24(L)=0$.

$25(L)=0$.

$26(L)=0$.

$Z 7(L)=0$.

$28(L)=0$

$29(L)=0$.

$Z+R(L)=0$.

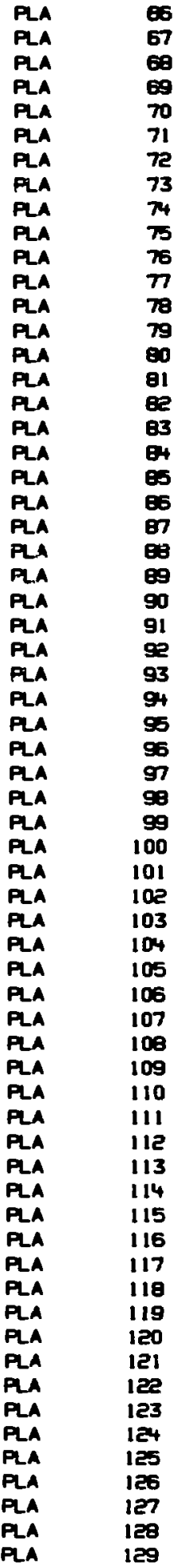


ZER(L)=0

ZSR $L$ ) $=0$.

WYAV $(L)=0$.

X

$S T E R(L)=0.0$

CXFAC (L) $=0.1)$

$\operatorname{SrAC}(L)=1.0$

$\operatorname{CFAC}(L)=1.0$

Crox(L) $=1.0$

$\operatorname{Cs}(L)=1.0$

$V B(L)=1.0$

$C \theta(L)=1.0$

100 CONT IME

HUYAL=0.0

SYAY $=0.0$

SFFA $=1.0$

CVTOT $=1.0$

AITT=0.0

SFPPEI0.0 $+F P / 2.0$

PLA 130

EFFP-210.04FFP/2.0

PLA

I3I

EFPAEl0.04FP/2.0

PLA 13

Pla 133

PLA 130

PLA 135

PLA 136

PLA 137

PLA $\quad 130$

PLA 130

PLA 140

PLA 141

PLA 140

PLA 143

PLA 144

PLA 145

FA $\quad 146$

FA 147

PLA $\quad 148$

CX000000000000000XIPRIGATION MOUnEX0000000000000000000000000000000000X PLA

C CUTX-HARVESTING DATES, ALTX-ANT HATER APFLIED.DIFX-IFR. SCHEDUL PLA CX000000000000000000000UTX(IXPL, 1$)$ STATERENTS RESERVD FOR HAPVEST OATEX PLA CX000000000000000000000IFX(IXI, 1$)$ STATEMENTS RESERVD FOR PLAMTING DATE PLA DO 110 L-1.2e

IF (RIFAC(L) .EO.0.0) CO TO 110

READ 1570, (CUTA(L,K),K=1,8)

READ 1570. (AHATA(L,K),K=I,16)

READ 1570, (DIRA(L,K),K=1, (6)

110 CONTIME

$c \times 0 \times 00000 \times 0000000000 \times 00000000000 \times 000 \times 00000000000000$ 17-RANDN

$A=(T 2-T 1) / 2.0$

$\theta=(T 4-T 3) / 2.0$

$T B=0.0$

DO $120 \quad 11=1,17$

120 YaPWDOM(e.0)

IJINT (Ye)

ICYR=1

Do 1500 1e-i.iJ

$11=12$

ICOY=1

$A \mid I=I I$

If IIYR.EO.OI CO TO 130

IF (AI I.EO. YRONT (ICYR)+1.0) ICYA=ICYR+1

IF IICYR.GT.IYRI ICYR=IYR

$130 \quad I 1=11-1$

IF (II.NE,O) CO TO 170

PRINT I500

PRINT 15:0

PRINT 1600, IJ

If INICHE.EO.O) PRINT 16IO. ANT

IF (NICE.GT.0I PRINT 1610, BLAK

If IRSCOE.EO.0.01 PRINT IGZO, ANT

IF (ASCOE.GT.0.0) PRINT IEZO. BLAK

IF ICYR.EO.0.01 PRINT 1630, MDT

IF ICYR.GT.0.0) PRINT 1G30, ELAK

IF (IRIG.EQ.0) PRINT IEHO, ADT

IF (IRIG.GT.0) PRINT IESO, ElaNK

IF IFFNA.EQ.0.0) PRINT IESO, ANT

IF (FFAN.GT.0.0) PRINT IESO, BLAK

IF (FRCT.EQ.0.0) PRINT I670, ANDT

IF (FRCT.GT.0.0) PRINT 1670. BLAK

IF (REERE.EQ.0.0) PRINT IGEO. MDT 
IF IFITE.OT.0.0) PRINT IEBO, BLAK

PRINT 1690

PRINT 1700

PRINT 1710, TNXV,T1,T2,G1

PRINT I720

PRINT 1730, T3, T4,L3

PRINT 1740

PRINT 1750

PRINT 1750, R.PFCV.FFP,EFP

PRINT 1:?0

PRINT 1780

PRINT 1790, $(x(1), 1=1,12)$

PRINT 1800

PRINT 1810, TRE

PRINT IEeO

PRINT 1830

PRINT IE4O, FI,H,WI, YI,SOPTH

D $140 \mathrm{~J}=\mathrm{l} .22$

IF (RSC(J).EQ.0.0) CO TO 140

PRINT 16so, J

PRINT 1EEO, $(21(J, L), L-1, J 1 C O E)$

PRINT 1800

PRINT 1800. (ZF (J,L),L-1,11COE)

PRINT 1870

PRINT 18E0, (ZUU(J.L),L-1,IICOE)

140 CONT inue

If (CYR.EQ.0.0) 00 TO 150

PRINT IESO

PRINT 1900

PRINT 1910, CRAC.CYR.DURC.CHFSY,CFEY

PRINT 19:0

PRINT 1930. CHRSO.CFRED

150 CONTINE

IF (IRIO.EQ.0I 00 TO 170

PRINT 1940

DO $160 \mathrm{~J}=1,22$

If (RIFAC(J) .EQ.0.0) CO TO 160

PRINT 19S0, J.ACRESIJ)

PRINT 1950

PRINT 1970. COIRA (J.1), 1=1.16)

PRINT ISO0

PRINT 1970. (ALATA $(J, 11,1=1,16$ )

PRINT 1900

PRINT 2000, (CUTA(J,1), $1=1,8)$

160 CONTINE

170 Contines

AO-II

K3=0

PI $=0.0$

TAY $=0.0$

DO $180 \mathrm{~L}=1,2 \mathrm{e}$

MRAT $(L)=0.0$

IRO(L) $=2$

ICA(L) $=2$

180 CONTINS

Do $1350111=1,350$

Al11-111

AlfCA $=0.0$

If (IVR.EO.0) CO to 190

If (A)I.NE. YRCNTIICYR) CO TO ISO

If (AIII.LT.DAICIICYR) CO TO 190

If (All1.GE.DAICIICYR)+DURC) CO TO 190

AIRCA-AMAC (ICYR, ICOY)

ICOY=1CDY+1

PLA 197

PLA 198

PLA 190

PlA 200

Pla 201

Pla 202

PLA 203

PLA 204

PLA 205

Pla zos

PLA 207

Pla 200

Pla 209

PLA 210

PLA 2II

FLA 2ID

PLA 213

PLA 214

PLA 215

PLA 216

PLA 217

PLA 218

PLA 219

PLA EZO

PLA 221

PLA zee

flA 223

PLA 2E4

PLA Z2S

PLA 2E6

PLA 2E?

PLA 2EQ

PLA 2E9

FLA 230

Fla 231

FLA 232

PLA 233

PLA 234

PLA 230

PLA 236

PLA 237

FLA 238

FLA 230

PLA 240

PLA 241

PLA 24?

PLA 243

PLA 244

PLA 245

PLA 246

FLA 247

PLA

PLA 249

PLA 250

PLA ESI

Pla zes

Pla 2EJ

PLA 204

PLA ENS

PLA ES

PLA 
190 IF (AII.LT.CHASY.OR.AII.GT.CHEY) CO TO 200 IF IAI I I.LT.CFASD.OR.AI I I.GT.CFED) SO TO 200 AIRCA=AIRCA+AIRAC CORAC.AII.AIIII I .OR. III.EQ. IS2.OR.III.EQ.18R.CR.111.EQ.213.OR.111.EQ.244.0R.111 2.EQ.E74.OR. JII.EQ.305.CR.JII.EQ.355) 210.280 $210 \mathrm{M}=0$

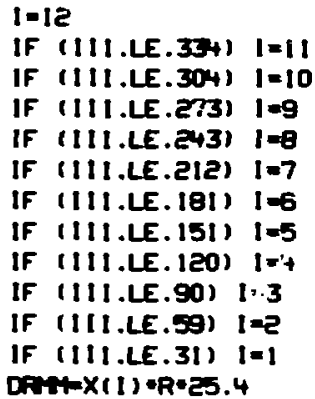


If (M.EO.NN(L)) CO TO $310 \quad$ PLA Je?

300 CONTINE

co to $\geq 00$

310 NOWNE

PuO(N) EOANices

Pupapegerficy

$3 \geq 0 \mathrm{PI}=\mathrm{PI} A \mathrm{P}$

If (T.OT.0.0) $\infty$ To 330

F7-74P

330 cont Inve

SAME 1 . 128*T

If (FT.LT.SNM) SNMA7

F7+7-SNy

E2-0.6*0.18・T

DO IEOO LFN" . 22

IF (ROC(LPN).EO.0.0) CO TO 1200

IF (T.OT.0.0) WA (LPN) $+H A(L P N)+P+S N Y$

PLA 323

PLA 3E4

PLA 3ES

PLA $3 E 6$

PLA 327

PLA $3 \geq 0$

PLA 329

PLA $\quad 330$

PLA 331

PLA 332

PLA 333

PLA 334

PLA 335

PLA 356

PLA 337

MM(LPN) $=A$ IFCA+MAR(IFN) PLA 330

CX000000000000000XIFRIGATION MODLEX00000000000000000000000000000000000X FLA 340

IF (RIRIC(LPN).EO.0.0) CO TO $300 \quad$ PLA

IRCCO=IRB(LPN) $\quad$ PLA

IF (AIII.NE.DIRALLN, IFCOG) ) CO TO 3+0 PLA

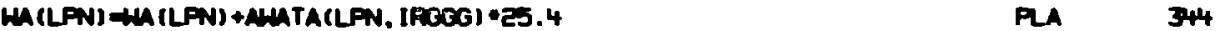

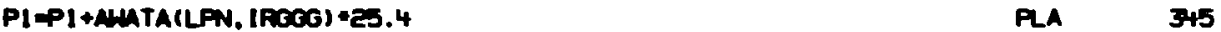

IRB(LPN) = IRG (LPNI+1 PLA $3+6$

3HO CONTIME $\quad$ PLA 347

CX000000000000000000000000000000000000000000000000000000000000000000000X PLA 3+8

IF (WM (LPN).GT.H) HA(LPN) HH PLA 349

EA(LFN) =EP.H (LPNI/FI PLA 350

IF (WACLN).LE.W1) EAILPN)=0.0 PLA

IF (WA(LPN).CE.FII EAILPN)=EP PLA 350

WA (LFN) WHA (LPN) EA (LPN) -SOILF (LFN) PLA 353

IF (WA(LPN).LE.HI) EA(LPN) WH(LPN)-HI+EA(LPN) PLA 304

IF (WH(LPN).LE.HI) HA(LPN) WHI PLA

SI WHA (LP) $\quad$ PLA 356

E4-EA(LFN) $\quad$ PLA 357

ISTP=1

SCTP(LPN)=1.0 PLA 300

SAPP-5.

ES(LPN) $1.0-(T-25.0) \cdot \cdot 2 / 255.0$

IF (T.GT.25.) ES(LFN)=1.0-(T-25.0)**2/81.0

IF (LPN.LE.10) CO TO 350

SAPP=3.

ES(LPN) $=1-(T-18) \cdot+2 / 256$

IF (T.LT.18.) ES(LPN)-1.0-(T-18.1・・2/169.

PLA $\quad 360$

PLA 361

PLA 362

PLA $\quad 363$

PLA 3FH

PLA 305

PLA 306

CX0000000000000000WRY SEASON PLANTS00000000000000000000000000000000000X PLA 367

350 CO TO $1350,370,300,330,400,410,420,430,440,450,460,470,480,490,500$ PLA 358

$1,510,520,530,540,550,560)$, LPN

350 Cont INUE

C COFN AND SORGHAM

CO TO 570

PLA $\quad 370$

PLA 371

370 CONTINUE

C ANUKL VECETABLS AD GRAINS

co TO 570

360 CONT INUE

C PASTURE GRASSES

co TO 570

300 CONT INE

C FRUIT TFEES

ES(LPN) $=1.0-(T-21.0) * 2 / 169.0$

IF (T.LT.21.0) ES(LPN) $=1-(T-21.0) *+2 / 121.0$

PLA 372

PLA 373

PLA 374

PLA 375

PLA 370

PLA $\quad 377$

PLA $\quad 378$

PLA $\quad 379$

PLA 380

PLA 391

ISTPuO

$\operatorname{SCTP}(L P N)=0.0$

PLA 382

CO TO 570

383

PLA 384

PLA 305 


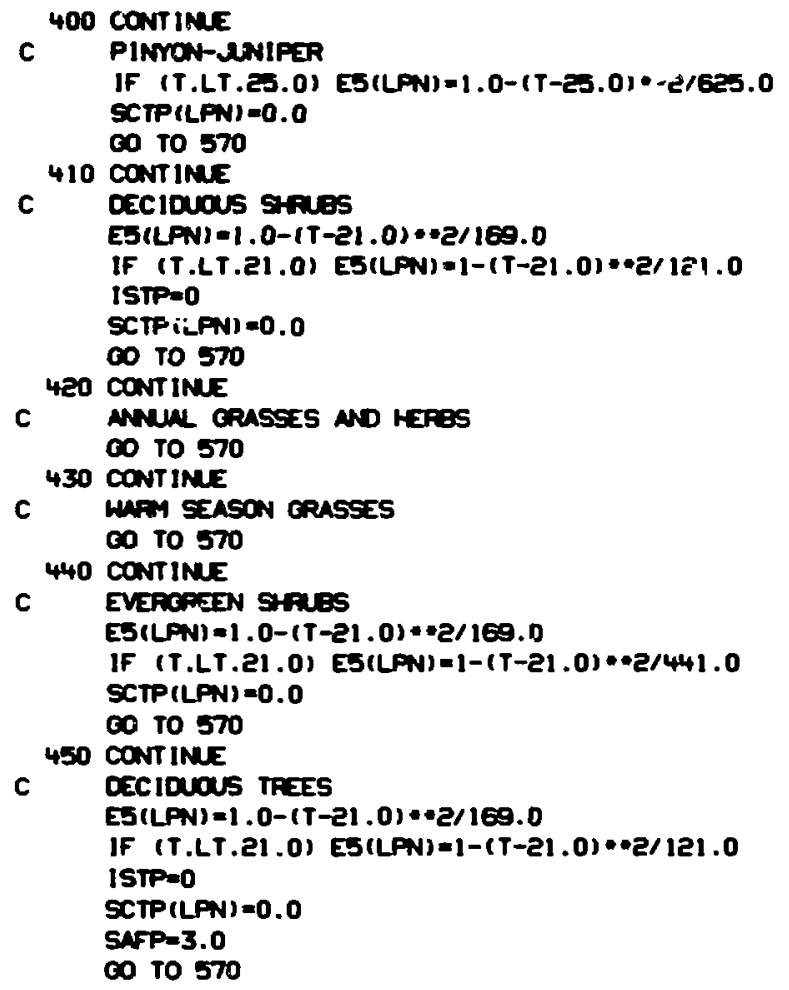

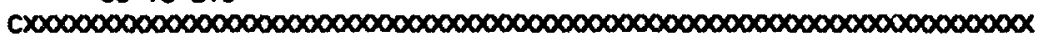
CX00000000000000001C00L SEASON FLANTS000000000000000000000000000000X 460 CONTINUE

C ALFALA

ES(LPN) $=1.0-(T-21.0) * 2 / 169.0$

IF (T.LT.21.0) ES(LPN) $=1-(T-21.0) *=2 / 121.0$

SAFP 4.0

CO TO 570

470 CONTINUE

C MNUAL VECETAELS AND GRAIMS CO TO 570

480 CONTINUE

C PASTURE GRASSES

CO TO 570

490 Cont INUE

C DOULAS FIR

ES(LPN) $=1-(T-14.0) * 2 / 400.0$

IF (T.LT.14.0) ES(LPN) $=1-(T-14.0) * 2 / 195.0$

SCTP (LPN) $=0.0$

CO TO 570

500 CONTINUE

C PONDEROSA PINE

If (T.LT.18.0) ES(LFN) $=1-(T-18.0) *-2 / 324.0$

$\operatorname{SCTP}(L F N)=0.0$

CO TO 570

510 CONTINUE

C DECIOUOUS SHFus

ES(LPN) $=1.0-(T-15) * 2 /$.

IF $(T . G T .15 .0)$ ES(LFN) $=1.0-(T-15.0) *-2 / 351.0$

ISTP=O

$\operatorname{SCTP}(L P N)=0.0$

CO TO 570 


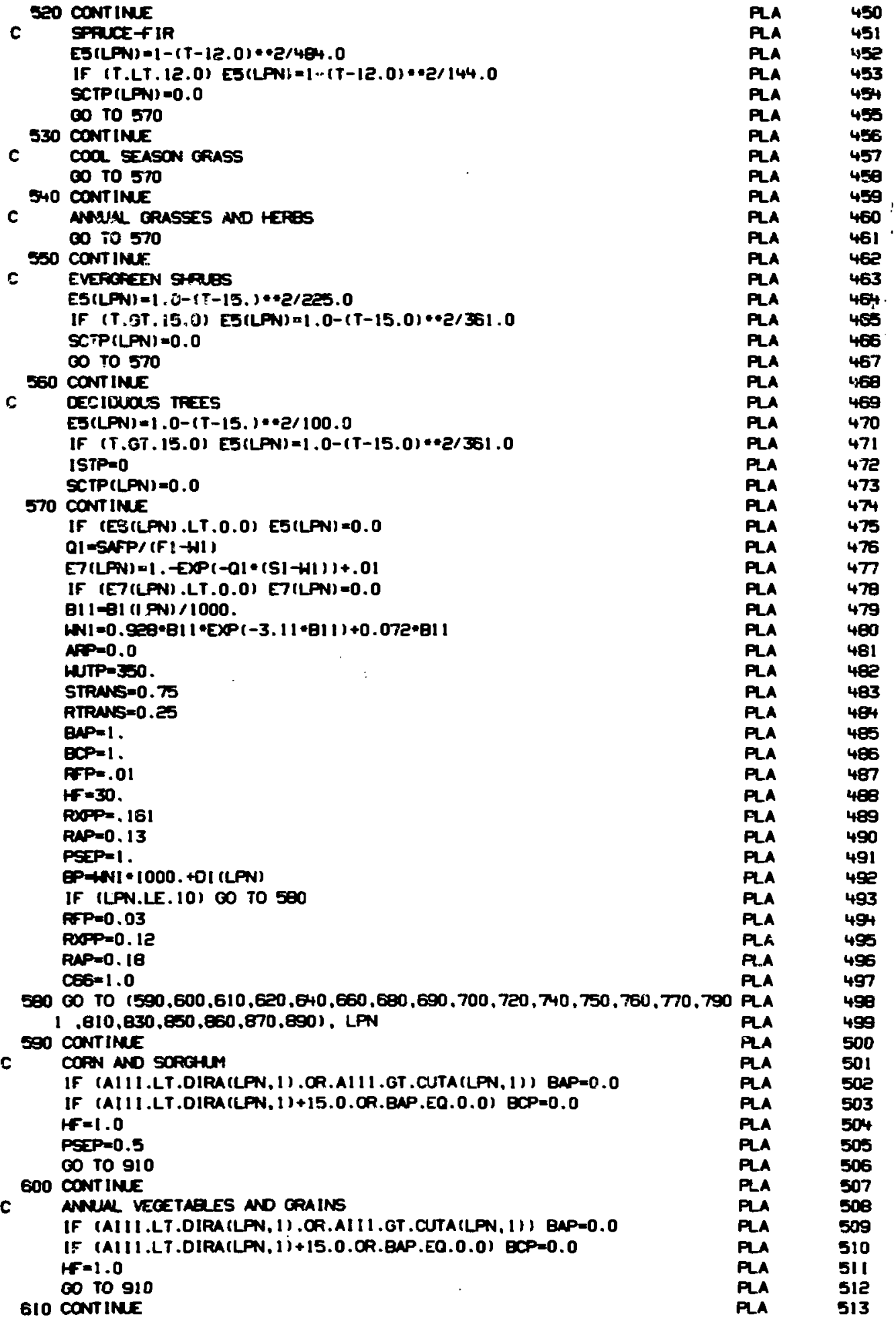


620 CONT INE

c FPUIT THEES

if (11.LE.OI CO TO 630

if (III.EQ. I) WNITHO(LPN)

IF (WN1.GE.0.5-TPCNW(LPN)) WN1-TPGNH(LPN)・0.5

630 AFP=1.5*WN1 1000 .

IF (AIII .LE.SFPP+1 . OR.AIII .GE.EFFP+1 .) ARP=ARP-WNI +1000.

IF (AFP.GT . BI (LPN) WN1 1000.) ARPABI (LPN) +WN 1000 .

$H F=150.0$

STRNST=0.85

RTRANS=0.15

EP=1000. * WN1

WUTP-900.0

Co To 910

640 CONTINE

c

PINTON-JUNIFER

IF (11.LE.0) CO TO 650

IF (111.EQ.1) WNI GNO(LPN)

IF (WNI.GE. TPCNAILPN) ) WNI = TPGNAILFN)

GSO AFP-5.0\%W1 +1000 .

IF (APP.GT.BI (LPN) WN1*1000.) AFP-BI (LPN) +NI 1000 .

VB(LPN) $=2.54$

CS(LPN $)=1.54$

$+F=300.0$

STRANS=0.85

RTRANS=0.15

EP=1000. ANI

WUTP=TES. 0

Co To 910

680 CONTINUE

C DECIDUOUS SHFuBs

If (II.LE.0) CO TO 670

IF III.EQ.1) WNI HWD(LPN)

IF (WN1 .GE.0.5-TPCNA!LPN)) LN1-TPCNH(LPN) 0.5

670 APP=2. *WN1*1000.

IF (AIII.LE.SFFP+1 ..OR.AIII.GE.EFFP+1 : AFP=ARP-WNI*1000.

IF (APP. GT .BI (LPN) WN1 * 1000.) AFP=BI (LPN) -WN1 1000.

$F=70.0$

$E P=1000.6 N 1$

HUTP-900.0

co TO 910

680 CONT INUE

C ANUA CRASSES AND HERBS

RTRAN5=0.5

STRANS $=0.5$

$r F=1.0$

co To 910

690 CONT INUE

c

WAPM SEASON GRASS

RTRANS $=0.7$

STRANS $=0.3$

HF $=30.0$

$\infty$ TO 910

700 CONT INUE

C EVERCFEEN SHRUES

IF (II.LE,0) CO TO 710

IF (III.EQ.1) WNI WND(IPN)

IF (WNI , GE. TPCNW(LPN)) WNI = TPGNNILPN)

710 APP $5.0 \%$ *N1 1000 .

IF (AFP.GT .BI (LPN) +WI +1000 . ) APP=BI (LPN) $-W N 1 * 1000$.

-2A

PLA

PLA

PLA

PLA

FA

Fu

Fu

FA

PLA

Fus

FuA

P.

Fu

Fu

PLA

FA

PLA

PLA

FLA

PLA

PLA

Pla

Fu

FL

PLA

PLA

PLA

PLA

FuA

FA

PLA

PLA

FuA

Pla

PLA

PLA

PLA

PLA

PLA

PLA

PLA

PLA

PLA

FLA

PLA

PLA

PLA

Pla

PA

PLA

Pla

PLA

PLA

PLA

PLA

PLA

FLA

PLA

PLA

FlA

PLA

PLA

PLA 
$K=70$.

EPI 1000. ONN1

Fla

MUTP=7E. 0

co to 990

720 cont inus

C

Deciouns Trass

IF (II.LE.OI CO TO 730

IF (III.EQ.1) WNI HWD(LFN)

IF (INI .CE.0.5•TPCNA(LPN)) WNI -TPCNW(LPN) *0.5

730 NFPl $1.5 *$ WON 1000

IF (AI11.LE.SPPP+1 . OR.AIII.CE.EFPP+1 . ) APPEAPP-WNI 1000 .

IF (APP.GT.EI (LPN) +NI 1000.$)$ APP-BI (LPN) +NI 1000.

EP=1000. *W1

STRMSO0.85

RTRENS=0. 15

HUTP-900.0

$K=175.0$

co 70910

740 cont INas

c

ALFALFA

STRNSAD.5

RTRNS=0.5

RPPE.01

ROPPE. 161

RAP. 13

co To 910

75O CONTINUS

$c$

ANAM VECETABLS ANO CRAINS

IF (AIII.LT.DIRACLPN, \&).CR.AIII.GT.CUTA (LPN, I I) BAP=0.0

IF (AIII .LT.DIRACLN, I )+15.0.OR.BNP.EQ.0.0) BCP=0.0

$H F=1.0$

CO TO 910

750 CONTINIS

c

PASTUEE GRASSES

RTRMS $=0.7$

STRNS $=0.3$

Co To 910

To CONTINe

c

DOUTLAS FIR

IF (II.LE.O) CO TO 700

IF $1111 . \mathrm{EO} .1) \mathrm{WN} 1 \mathrm{HWD(LFN)}$

IF (WN1 . CE. TPGN(LPN)) WNI-TPGN(LFN)

i80 AFPLE.0\%LN1 1000 .

IF (APP.GT.EI (LPN) - WNI*1000.) APP-BI (LPN) - WN1 1000 .

STRMNS=0.85

RTRANS=0.15

$H=310$.

EPLW1 1000 .

WUP=TES. 0

CO TO 910

750 CONTINE

c

ponozrosa pine

IF (II.LE.0) CO TO 800

IF (111.EP.1) WNI WND(LFN)

IF (LN1 .CE. TPGNU(LPN)) WNI-TPGN(LPN)

800 APPE 3. * *NI 1000.

IF (ARP.GT . BI (LPN) -WNI*1000.) APP-EI (LPN) -WNI *1000

Va(LFN) $=1.36$

STRANS-0.85

RTRANS=0.15

$H F=300$.

EPAN1 1000 .

WUP-7ES. 0

FPP $=01$

Pu

PLA 500

PLA EBI

PLA 5:e

PLA

Pla bor

Pla ses

PLA 505

PLA 5e?

Pla 580

PLA 500

PLA 590

PLA 591

PA

PLA 503

Pla 504

PLA

pla 5es

PLA 597

PLA 598

PLA 590

PLA 600

PLA 601

PLA 602

Pla 603

PLA 604

Pla 605

PLA 606

PLA 607

PLA 603

PLA 609

Fla 610

PLA GII

FLA GI2

Pla 613

PLA 614

PLA GI5

Pla

PLA 617

Fla GIE

PLA 619

PLA $\quad 620$

Pla E21

Pla Ge?

PLA 623

PLA E:4

PLA EZS

PLA ges

ALA 627

PLA $6 E 0$

PLA GES

PLA $\quad 630$

PLA 631

Pla 632

PLA 633

PLA 634

PLA 635

PLA 635

PLA 637

PLA 638

PLA 630

PLA GHo

PLA GHI 


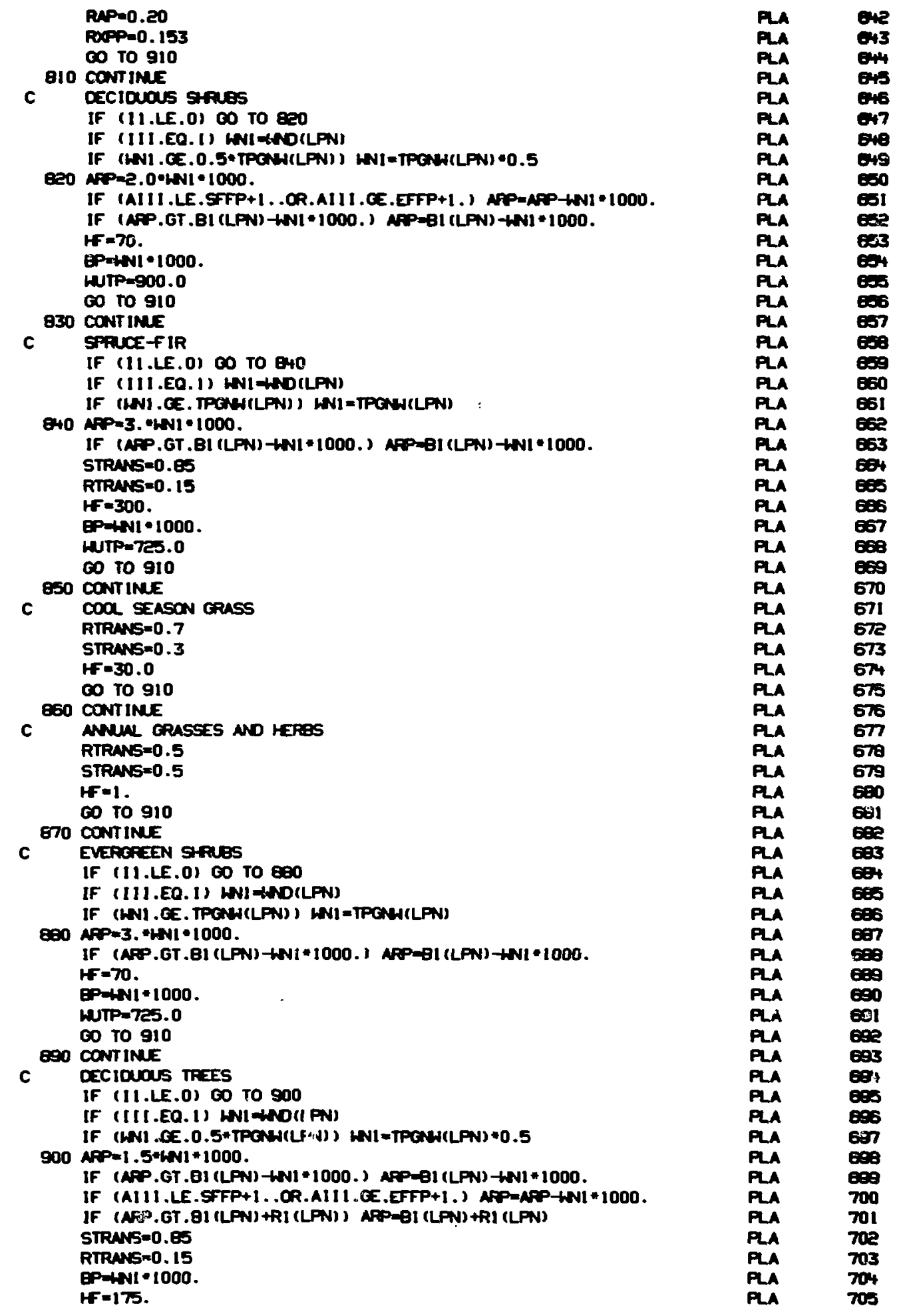


MUTP-900.0

910 CONTIME

AC=.3०RFP-EXP (ROPP.T) $T$ RAP

$A C=A C \cdot S F A C$ (LPN) $=C F A C$ (LPN)

WNI 4 NI 1000.0

TLAI (LPN) $=0.013^{\circ} E P$

RSPIO(LPN) $=$ APP

IF (APP.EO.0.0) RSBIO(LPN) WNI

PSE-PSEP

IF (RSCDE.EQ.0.0) SFAC(LPN) $=1.0$

If (RSSILPN).GT.12.) SFAC(LPN) $=1.0$

If (RSCDE.GT.0.0.ANO.11.LE.0) SFAC(LPN) $=(30 . / \mathrm{HF}) /$ RSCDE

If IISTP.EO. II CO TO 920

IF (AIII.GT.SFFP+1.A.All1.LT.EFFP+1) 60 TO 920

HN $1=1.0$

S20 BS(LPN) HNI *BAP+MI *ES (LPN) *E7 (LPN)

BS (LPN) - BS (LPN) - (LNI +RPP) *RFP *EXP (RXPP * T) *RAP BBCP

ES(LPN) $-B S(L P N)=C F A C$ (LPN) + SFAC (LPN)

T5-64- 1000

TS= TS-CFAC (LPN) = SFAC (LPN)

WNO(LPN) -WNI

TSP (LPN) $=$ TS

C-EST(LPN) EET (LPN)

IF (C.LE. O.01) C=0.01

CAUIP/C

$C=C+P S E$

TG-C०ES(LPN)

EUTT-ES(LPN)

If (T6.GT.T5) EUAT=(T5/T6) $=85$ (LPN)

If (BSILPN).LE. 0.0) EWAT-ES(LPN)

WURAT (LPN) GWRAT (LPN) +EWAT

BS(LPN)-EWAT

B7 (LPN) MRTRASE-BS (LPN)

BS(LPN) - ES (LPN) +STRANS

IF (ESILPN).GE.0.0) CO TO 930

QS(LPN) -ES (LPN) /STRANS

G7(LPN) $=E 5(L P N)+R I$ (LPN) / (BI (LPN) +RI (LPN))

BS(LPN) $=E S$ (LPN) - BT (LPN)

930 contine

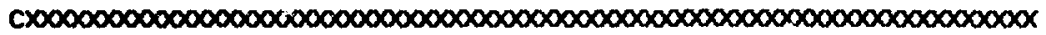

B1 (LPN) $=81$ (LPN) +ES(LPN)

R) (LPN) -R1 (LPN) +B7 (LPN)

RSCF (LPN) If

AP=2./ (305. -HF)

PLA 706

PLA 707

PLA 700

PLA 709

Fla 710

PLA 711

PLA 712

PLA 713

PLA 714

PLA 715

PLA 716

PLA 717

FlA 718

PLA 719

PLA 720

PLA 721

PLA TE2

PLA 723

PLA 7e4

PLA 7ES

PLA TES

PLA 727

PLA 729

PLA 7Eg

PLA 730

PLA 731

PLA 732

PLA 733

PLA 734

PLA 735

PLA 736

PLA 737

PLA 738

PLA 730

PLA $\quad 740$

PLA 741

PLA 742

PLA 743

PLA 744

PLA 745

PLA $7+6$

PLA 747

PLA 749

PLA $\quad 749$

CO To $(940,950,980,990,1000,1010,1020,1030,1040,1050,1050,1070,109$ PLA 750

$10,1100,1110,1120,1130,1140,1150,1160,1170)$, LPN

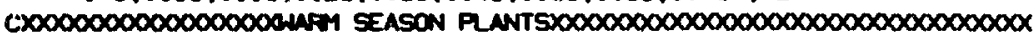

940 CONTINUE

C COFN ANO SOREAM

D3=.005*B1 (LFN)

IF (T.GT.25.) DJ-91 (LPN)*(.G5*(T-25.)+.10)*.05

IF (T.LT. 10.) DJ-B1 (LPN) $=(1.1-.1=T)=.05$

RO3-03*R1 (LPN)/BI (LPN)

IF (AIII.NE.CUTALPN, 1 ) CO TO 950

$24(L P N)=24$ (LPN) +0. 1 - Z3 (LPN)

PLA 751

PLA 750

PLA 753

FLA 754

PLA TOS

PLA 756

PLA $\quad 757$

PLA TSO

PLA 750

PLA 750

$Z 3(L P N)=0.0$

$03-91$ (LPN) 0.1

$Z W R(L F N)=Z 4 R(L P N)+Z 2(L P N)$

PLA TEI

PLA TES

$Z C(L P N)=0.0$

RO3-RI (LPN)

PLA 763

PLA 704

PLA 705

PLA 705

PLA 767

$B($ (LPN) $=1$.

R1 (LPN) $=1$.

PLA 708

ES(LPN) $=0.0$

PLA 709 


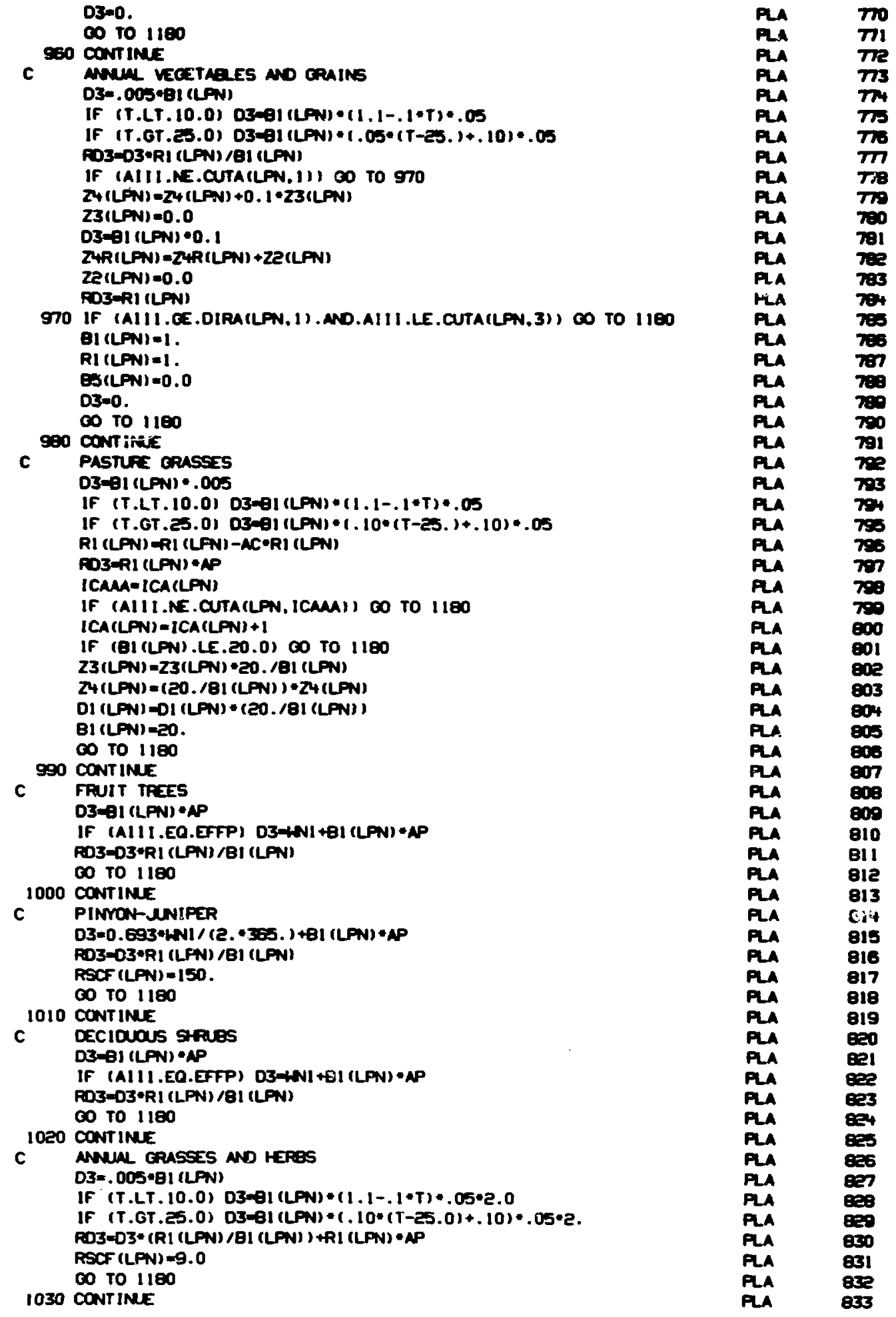


IF (T.LT.10.0) DS-OI (LFN) $(1.1-.1 \cdot T) \cdot .05$

IF (T.OT.25.0) DS-81 (LPN) * . 10・(T-25. )+.10) ..05

RI (LPN) $=R 1$ (LPN) - AC*RI (LPN)

RDZARI (LPN) $\triangle A P$

60 to 1100

1040 CONTINE

c Evakizan siruas

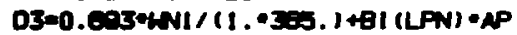

RO3-03-RI (LPN) / 1 I (LPN)

60 To 1180

10so contine

C DECIOUOS TREES

D3 $A$ NPEI (LPN)

IF (AI11.EO.EFPP) O3-WN1 +Q1 (LPN) $=A P$

RO3 $03+R$ ) (LPN) /QI (LPN)

co To 1180

CX000000000000000000L SEASON FLANTSO0000000000000000000000000000000X IOSO CONTINLE

C ALALA

D3=.00J・BI (LPN)

IF $(T . L T .7 .0) \mathrm{DB}-\mathrm{BI}(\mathrm{LPN}) *(1.1-.14 * \mathrm{~T}) * .05$

IF (T.GT.21.01 03-BI (LPN)*1.05*(T-21.)+.10)*.05

R1 (LPN) =RI (LPN)-AC•RI (LPN)

ROS-R1 (LPN) AAP

ICAMEICA (LPN)

IF (AIII.NE.CUTACLN. ICMAA) CO TO 1180

ICA (LPN) $=I C A(L P N)+1$

IF (EI (LPN).LE.EO.0) CO TO 1180

$Z 3(L P N)=Z 3(L P N)=20 . / \theta 1$ (LPN)

$24(L P N)=(20.181$ (LPN) $)=Z 4$ (LPN)

D1 (LPN) $\Rightarrow 1$ (LPN) $=(20.181$ (LPN) )

BI (LFN) $=20$.

co to 1180

1070 contine

C ANULL VECETAELES AND GAINS

D3-.005*81 (LPN)

IF (T.LT.7.0) D3-BI (LPN)*(1.1-.14・T)・.05

IF (T.GT.21.0) D3-B1 (LPN) $(.05 *(T-21)+.10) *$.

RD3-03*RI (LPN) /BI (LFN)

IF (AIII.NE.CUTAILPN, 11$)$ CO TO 1080

Z3(LPN) $=0.0$

D3-BI (LPN) 0. 1

$Z Z(L F N)=0.0$

RO3-R1 (LPN)

1000 IF (AI1).GE.DIRAILPN, 1).AND.AIII.LE.CUTA(LPN,1) 60 TO 1180

$\mathrm{B} 1(\mathrm{LPN})=1$.

$R 1(L F N)=1$.

ES(LPN) $=0.0$

$03=0$.

CO TO 1180

1090 CONT INE

C PASTURE GRASSES

03- .005*81 (LPN)

IF (T.GT.18.0) 03-81 (LPN) $\div(.10 *(T-18.1+.10) * .05$

IF (T.L.T.5.0) 03-91 (LPN)*(1.1-.2*T)*.05

R1 (LPN) =R1 (LPN)-AC*R1 (LPN)

FOSTRI (LFN) • AP

ICAMA ICA (LPN)

IF (AIII.NE.CUTA(LPN, ICNA)) CO TO 1180

ICA (LPN) $=I C A$ (LPN) +1

IF (BI (LPN).LE.BO.) 60 TO 1180

$Z 3(L P N)=Z 3$ (LPN) *20. /G1 (LPN) 
$24(L P N)=24(L P N) *(20 . / 81$ (LPN))

ma

01 (LPN) $=01$ (LPN) * (20. / 1 I (LPN) )

PLA geg

BI (LPN) $=20$.

CO TO 1180

1100 cont ine

c DOUCLAS FIR

D3-0.603-WN1 / IE. A3E5. ) +QI (LPN) * AP

FD3-D3-RI (LFNI/BI (LPN)

CO TO 1180

1110 CONTINUE

C PONDEROSA PINE

D3=0. ES3*WNI $/(2 .-365)+.Q 1(L P N)=A P$

P

PLA 9o!

PLA SOE

PLA $\quad 903$

PLA 904

PLA 905

PLA 905

PLA 507

RO3-D3-RI (LPN)/BI (LPN)

RSCF (LPN) $=150$

co To 1180

1120 CONTINE

C OECIOUOUS gRIBS

DB-OI (LFN) *AP

IF (AIII.EO.EFFP) D3=WI+BI (LPN) = AP

PLA

PLA

PLA 910

PLA 911

PLA gre

PLA 913

Pla 914

PLA 915

PLA 916

ROZ $-D 3 \bullet R I$ (LFN) /BI (LPN)

CO TO 1180

1130 CONTINE

C SPRUCEFIR

D3=0.693+WWI / (2. -365. )+QI (LPN) + AP

RD3=D3-R1 (LPN) / 1 1 (LPN)

CO TO 1180

1140 CONTINUE

C COO SEASON GRASS

D3-. $005 \cdot B 1$ (LPN)

IF (T.LT.5.0) O3-B1 (LPN) $(1.1-.2 * T) \bullet .05$

1F $(T, G T .18 .0)$ O3-81(LPN) $\cdot(.10 *(T-18)+.10) *$.

PLA 917

PLA 918

PLA 919

PLA $\quad$ geO

PLA SEI

Pla gez

PLA 923

PLA Sa

PLA

PLA ges

PLA Se?

PLA SES

R1 (LPN) -R1 (LPN) - AC -R1 (LPN)

RO3-R1 (LPN) $=A P$

CO To 1180

1150 CONTINUE

C ANUNL GRASSES AND HERBS

$03=.005 \cdot B 1$ (LPN)

IF (T.LT.5.0) D3-BI (LPN)*(1.1-.2*T)*.05*2.

IF (T.GT.18.Q) D3=B1 (LPN)*(.10*(T-18.)+.10)*.05*2.

PO3-OB-RI (LPN) / II (LPN) +RI (LPN) = AP

RSCF (LPN) $=9.0$

CO TO 1180

I.60 CONT INUE

C EVERTFEN SATES

D3-0.693*HN1 / (1 . -385. ) +BI (LPN) - AP

RDS=D3*RI (LPN) $/ B 1$ (LPN)

CO TO $: 180$

1170 CONT INUE

C OECIDOUS TREES

O3-B1 (LPN) $* A P$

IF (AIII.EO.EFFP) D3-WNI+QI (LFN) - AP

RO3-03•RI (LFNI/BI (LPN)

1180 CONT INE

IF (BI (LPN)-D3.LE. 1.0) D3=0.0

IF (R1 (LPN) -RD3.LE. 1.0) RD3=0.

B1 (LPN) $=$ B1 (LPN) -03

Ri (LPN) $=R 1$ (LPN) - RO3

D1 (LPN) $=$ DI (LPN) +03

FD1 ‘LPN) $=$ FD1 (LPN) +FO3

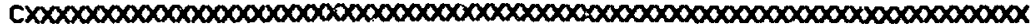

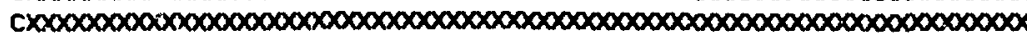

HE=.004+.003E-T

$H 3=1.0+(1.0 /(F 1-H 1))=($ HA (LPN) $-W 1)$

$H 1(L P N)+H 1$ (LPN) +HE*HB=DI (LPN)

PLA ges

PLA $\quad 930$

PLA 931

PLA QZe

PLA 933

Pla 934

OLA 9Bo

PLA 930

PLA 957

Pla 930

PLA 980

PLA $\$ 40$

PLA 941

PLA 942

PLA 943

PLA

PLA 945

PLA 946

PLA 947

PLA 949

PLA 949

PLA SSO

PLA SSI

PLA

PLA

PLA 504

PLA SE

PLA SOS

PLA 507

PLA SOE

PLA 900

PLA 980

PLA gBI 
FHI (LPN) ARHI (LPN) HE.H(Z•RDI (LPN)

DI (LPN) $D 1$ (LPN) + E•HB॰DI (LPN)

ROI (LPN) $=F O 1$ (LPN) +E•H(B॰RDI (LPN)

HI (LPN) HII (LPN)-AC*HI (LPN)

FHI (LPN) -FHI (LPN) -AC •RHI (LPN)

If (BL (LPN).LE.1.0) Bl (LPN) $=1.0$

IF (RI (LPN) .LE.1.0) RI (LPN) $=1.0$

IF (DI (LPN).LE.1.0) DI (LPN) $=1.0$

IF (ROI (LPN).LE.1.0) ROI (LPN) $=1.0$

If (HI (LPN).LE, 1.0) HI (LPN) $=1.0$

If (FHI (LPN).LE.1.0) FHI (LPN) $=1.0$

IF $(11 . E Q .0 .0)$ co to 1190

IF (ROCC(LPN).NE.3.0) CO TO 1190

IF (BI (LPN).LE.1.0) CO TO 1190

IF (II.NE.XPLNT) $\infty$ TO 1190

CALL FOPMAN (I:I,LPN, 29(LPN), MMA(LPN) )

1190 CONTINE

1200 CONTIMUE

IF (FHEF.EQ.1.0) CAL RANUNT (11,111.AITT,XERE)

IF (NICE.EQ.0) CO TO 1240

CX00000000000000000000000X NICHE CONPETITION $\times 0000000000000000000000000000 X$ CO $1220 \mathrm{k}=1,22$

CFAC (K) $=1.0$

IF (RSS(K).EQ.0.0) CO TO 1220

IF (BS(K).LE.0.0) CO TO IEeO

CFAC (K) $=(E S(K)+E 7(K)) \cdot C B(K) /(R S C F(K) * W N D(K))$

CXFAC (K) - CFAC (K)

DO $1210 \mathrm{~L}-1.22$

IF (RSS(L).EO. O.O.OR.K.EO.L) $\infty$ TO 1210

IF (BSIL).LE.0.0) Co TO IEIO

IF (RSSILI.NE.RSS(K)) CO TO 1210

CFAC (K) -CFAC (K) + (BS(L) +ET(L)) *CS (L) / (RSCF (L) *WN(L))

1210 CONT INUE

1220 contine

DO $1230 \mathrm{~K}=1.22$

If (RSSIK).EO.0.0) CO TO 1230

If (ES(K).LE.0.0) co TO 1230

CFAC (K) $=C X F A C(K) / C F A C(K)$

1230 CONTINE

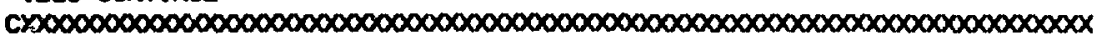

1240 CONTINLE

CVTT $=0.0$

AlTT $=0.0$

DO $1250 \mathrm{~L}=1.2 \mathrm{C}$

IF (ROC(L).EQ.0.0) $\infty$ TO 1250

IF (RSCOE.EQ.0.0) CO TO 1260

CVTT-CVTT+CCVV $(L)=C$ VIR (L)

AITTEAI TT+AI IL) =CVipd (L)

IESO CONTINUE

IF (CVTT.GT.1.0) CVTT=1.0

RSUSPILPN) $=2.5^{\circ}$ EXP $\left(-4.6^{*} \mathrm{CVTT}\right) * 1 . E-C B$

1260 DC 1310 LPN=1.22

If (REC(LPN).EO.0.0) CO TO 1310

RSUSP (LPN) $=2.5+E X P\left(-4.6{ }^{\circ} \mathrm{CVTT}\right)+1.0 E-00$

IF (CVTT.NE.0.0) 00 TO 1270

AITT-AI (LPN)

CVTT-CCWV(LPN)

RSUSP(LPN) $=2.5+E X P(-4.6 * C V T T) * 1 . E-C O$

1270 cont INUE

If (P.GT.0.0) CO TO IZEO

Z1 (LPN, 1)-Z1 (LPN. 1)-RSUSP (LPN) - Z1 (LPN, 1) - $1.693 / 700$. ) $+Z 1$ (LPN. 1 )

Z1 (LPN, 1)=Z1 (LPN, 1)+.0021*A1TT*(1.0-CVTT)/3.0EO4

$Z$ : (LPN, 2) *Z1 (LPN, 2) + . .693/700. ) *Z1 (LPN, 1)*3.0E04/3.75EDS

A1Z-RSUSP (LPN) 21 (LPN. 1)*3.0EO4

PLA gse

PLA 983

Pla Sar

PLA Gos

PLA SES

PLA 987

PLA 980

PLA $\operatorname{css}$

PLA $\quad$ gro

FLA STI

PLA STE

PLA 973

PLA 974

PLA 975

PLA 976

PLA $\quad 977$

PLA 979

RLA 979

PLA $\quad$ SBO

PLA SaI

PLA gaz

PLA 993

PLA gor

PLA 900

PLA

PLA 997

PLA $\quad$ gBg

PLA 989

PLA 990

PLA S91

PLA 980

PLA 993

PLA 9S4

PLA 905

PLA gOS

PLA 997

PLA $\quad 998$

PLA 989

PLA 1000

1001

$\begin{array}{ll}\text { PLA } & 1002 \\ \text { PLA } & 1003\end{array}$

PLA 1004

PLA 1005

PLA 1006

PLA $\quad 1007$

PLA $\quad 1008$

PLA $\quad 1009$

PLA 1010

PLA 1011

Pla 101 e

PLA 1013

PLA 1014

PLA 1015

FLA 1016

FLA 1017

PLA 1018

PLA 1019

PLA 1020

FLA 1021

PLA 10Ee

PLA 10 Ja

PLA 1024

Pla IOES 


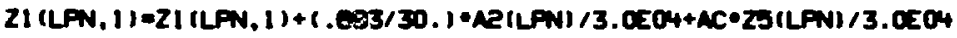
A1 (LPN) -ASUEP (LPN) * (AD (LPN) + ZS(LFN) I + A12

IF (MACLN).OT.0.0) AI (LFN)=AI (LPN)+ MA(LFN)

MACLN) $=0.0$

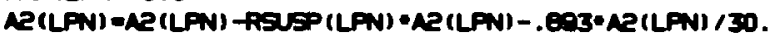

$A 2(L P N)=A 2(L P N)+.0021 \cdot A I T T \cdot T L A I(L P N) \cdot C V T T \cdot .67$

CO TO 1200

1280 A! (LPN) $=0.0$

21 (LPN, 1$)=21$ (LPN, 1$)+.3 * A 2$ (LPN) $=(1 .-E X P(-.0518 \circ P)) / 3 . E O 4$

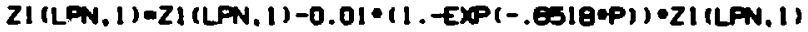

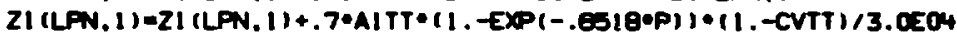

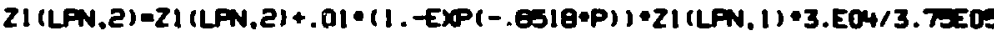

$A 2(L P N)=A 2(L P N)-.3 * A 2(L P N) \bullet\left(1\right.$. EXP $\left(-.05 \mid \theta^{\circ} * P\right)$

$A 2(L P N)=A 2(L P N)+.7 \cdot A \mid T T \cdot(1$. EXP $1-.651 \theta \cdot P)) \cdot C V T T \cdot T L A I$ (LPN)

PLA

PLA

1009

PLA 1031

PLA 1032

1290 CONTINE

$A|I P=(A \mid 1-1,) \cdot 320 .+A| 1 \mid$

IF (RSOF (LPN) .LE.9.0) AIIP=AIII

FPNTP= (1 . EXP $(-.0001 * A \mid$ IP) ) 99144 .

$11 C 0 E=I N T$ (RCOOE (LPN) /ESO.0)+1 .0

DO 1300 LES, IIOOE

ALLE-Z

$\operatorname{SP} 1.0$

IF (FPNTP.LT.A.AESO.) SP=0.0

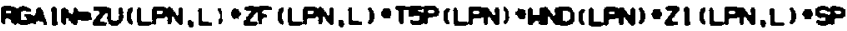

ZZ $(L P N)=Z Z$ (LPN) FAGAIN

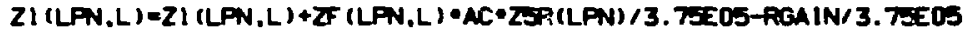

PLA

Pla

1033

PLA 1030

PA 1030

1037

PL 1033

Fu 1030

PLA 1040

PLA 1041

Pla 104?

PLA 1043

Pla 1044

Fu 1045

FLA 1046

PLA 1047

PLA 1048

PLA $\quad 1049$

PLA IOSO

PLA 1051

1300 CONTINES

$H B=1.0+(1.0 /(F)-W 1))=(H A(L P N)-W 1)$

ZP=1.

IF (Z) (LPN, 2$), G T .0 .0) Z P=Z 1$ (LPN, 2$)$

B7(LPN) =AES (BT (LPN))

IF (BT (LPN) .GT.RSBIO(LPN)-I .0) B7(LPN) ARSBIO(LFN)

BIII-BI (LPN)

RII I RI (LFN)

T111-RSBIO(LPN) HAD (LPN)

$A D D=(B 7(L P N) / T 111) \cdot(1 .-B 7(L P N) / T 111)$

IF (eI (LPN).LE.1.) NOO=0.0

ZZLPN=ZZ (LPN)

ZZLFN=Z3 (LPN)

$Z 2(L P N)=Z 2(L P N)-A D D \cdot Z 2(L P N) \cdot B 111 /(B 111+R 111)$

$Z 2(L P N)=Z Z(L P N)+A D D+Z 3(L P N)+R 111 /(B 111+R 111)$

$Z 3(L P N)=Z 3(L P N)+A D O \cdot Z 2(L P N) \cdot B 111 /(R 111+B 111)$

$Z 3(L P N)=Z 3(L P N)-A D D \cdot Z 3(L P N) \cdot R 111 /(R 111+B 111)$

ZZLPNTZZ(LPN)

23 PN=Z3 (LPN)

23 (LPN) $=23$ (LPN) -23 (LPN) $=03 / 81$ (LPN)

$Z 2(L F N)=Z 2(L P N)-Z Z(L P N) \cdot R 03 / R 1$ (LPN)

IF (ZE(LPN) .LT.0.0) ZZ(LPN) $=0.0$

IF $(Z 3(L F N), L T .0 .0) Z 3(L P N)=0.0$

$Z 4 L F N=Z 4$ (LPN)

Z4RLPN-ZUR(LPN)

ZOLPN=25(LPN)

ZSREPN=ZSR(LPN)

$Z+(L P N)=Z 4(L P N)+Z 3$ (LPN) $-D 3 / B 1$ (LPN) +2"H3- Z4 (LPN)

$Z 4 R(L P N)=Z 4 R(L P N)+Z 2(L P N)=F .03 / R 1(L P N)+E * H 3=Z 4 R(L P N)$

PLA

FLA

PLA

PLA

FLA

PuA

FuA

FL:

PLA

PLA

Fu

PLA

PLA

PLA

PLA

PLA

PLA

PLA

Fla

1052

1053

1004

1005

105

1007

1003

$10=3$

$$
1060
$$

1061

1050

1053

1054

1005

1055

1057

105

1000

1070

PLA 1071

PLA 1072

PLA 1073

PLA 1074

PLA 1075

PLA 1075

PLA 1077

PLA 1078

PLA 1079

$Z 9(L P N)=Z 9(L P N)+A C=Z 5$ (LPN)

$Z 9 R(L P N)=Z 9 R(L P N)+A C=Z S R(L P N)$

Z5 (LPN) -ZS (LPN) +

FA

Fla

1000

Fu

FLA

FLA

PLA

$Z 6(L P N)=Z 2(L P N) / R 1$ (LPN)

$X B(L F N)=Z 6(L F N) / Z P$

$Z 7(L P N)=Z 3$ (LPN) /BI (LPN)

$Z 8(L P N)=Z 7(L P N) / Z P$

PLA

PLA

PLA

TB-K3+365-11

PLA 
1310 contines

If in.NE.XFNTI CO TO 1350

PLA $\quad 1090$

PRINT EOIO. All

PAINT EDEO

Do I3E0 LPN-1, 22

If (AOCLILN).EQ.0.01 00 TO 1320

PAINT 2030, 91 (LPN), RI (LPN) .DI (LPN), HI (LPN) . ROI (LPN), RHI (LPN), T ,P

1 ,Al (LPN), ZP, TS,Al11,LPN

1320 continus

PAINT 2040

Do 1300 LPAN I. 25

If (mocllovi).eQ.0.0) co to 1330

PRINT 2030. A2ILPN), Z2 (LPN), Z3 (LPN), Z4 (LPN), ZUR (LPN), Z5 (LPN), ZSR

1 (LPN) , Z6(LPN) , Z7 (LPN), ZB(LPN), Z9ILPN), ZSR (LPN), LPN

1330 Contins

PRINT 2070

DO 1340 LON=1,22

IF (FCCLPN).EQ.0.0) CO TO 1340

27P=0.0

ZTPR=0.0

IF (OI (LPN) .0T.0.0) 2TP-Z4 (LPN)/DI (LPN)

IF (FO1 (LPN) . OT.0.01 ZTFR-Z4RILPN) /FOI (LPN)

AEZ-AZ(LPN)/EP

123-125/Z3

$Z T 7=(Z 7$ (LPN) +AE (LPN) /BI (LPN) )/ZP

FRINT 2030, ZTT, ZTP, ZTPR,XBILPN), AE2, A23, 21 (LPN, 11,21 (LPN, 2$), C F A C$

I (LPN). SFAC (LPN), LPN

1340 continue

1300 CONTINe

SYAV-SYAV+1.0

DO 1330 LPNWI, Ze

IF (ROC (LPN) .EQ.0.0) CO TO 1360

WO(LPN) $=$ WN D (LPN) $/ 1000$.

BII BI (LPN) $/ 1000$.

HII-.928*EXP $(-3.11 \cdot 811)+.072 \cdot B 11$

LUYNH-WPRAT (LPN)/1000.

IF (HII.LT.WUPAW.AND.SCTP(LPN).EO. O.0) HYYAWWW11

WYAV (LPN) GYYAV (LPN) + HUYAW

TPQNHILPN) HYYAV (LPN) /SYAV

IF (TPGNILPN) .LT.0.001) TPONH(LPN) $=0.001$

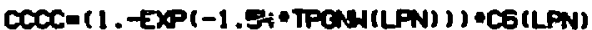

IF (CCCC. BT.CCWV(LPN). AND. ROC (LPN).EQ.2.0) CCW (LPN) =CCOC

IF (CBILPN).EQ.1.0) CCWV (LPN) $=C C C C$

1330 cont INUE

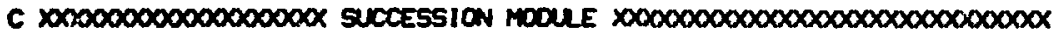

SFFC $=1.0$

IF (RSCOE.EO.D.0) CO TO 1490

DO $1370 \mathrm{~K}=1,22$

IF (FCC(K).EQ.0.0) 60 TO 1370

If (RSS(K).GT.12.) 60 TO 1370

RAK(K) $=1.0$

$\operatorname{STER}(K)=S \operatorname{STER}(K) * 10$

STER (K) -STER(K) + (QI (K) +Rl (K)) /RSCF (K)

STER(K) =STER(K) + (O) (K) +RDI (K) +HI (K) +FH (K) ) RSSCF (K)

STER(K) $=S T E R(K) /(A O+1.0)$

CVPX(K)-STER(K)

ACRES(K) - CCW (K)

1370 CONTINE

D 1300 L-1.22

If (RAK(L) EQ E. O. O.CR.RSS(L) .EQ.0.0) CO TO 1390

$\operatorname{CoV}|\operatorname{Cc} V(L) \cdot(\theta)(L)+R|(L))$

COVT-BI (L) +RI (L)

D $1330 \mathrm{~K}=1,22$

IF (RAK (K) .EQ O.O.OR.RSS(K).EQ.0.01 CO TO 1390

PLA 109

PLA 109

PLA $\quad 1093$

PLA 1094

PLA 1050

PLA 1005

PLA 109

PLA 1093

PLA $\quad 109$

PLA 1100

PLA 1101

PLA 1102

PLA 1103

PLA 1104

PLA 1105

PLA 1106

PLA 1107

PLA 1109

PLA 1109

PLA 1110

PLA 1111

PLA 1112

PLA 1113

PLA 1114

PLA 1115

FLA 1116

FLA 1117

PLA 1110

PLA 1119

PLA 1120

PLA 1121

PLA 1122

FLA 1123

PLA 1124

PLA 1125

PLA 1126

PLA 1127

Pla IIEE

PLA IIZ9

PLA $\quad 1130$

PLA 1131

PLA 1132

PLA 1133

PLA 1134

PLA 1135

PLA 1135

PLA 1137

PLA 1139

PLA 1139

PLA $\quad 1140$

PIA 1141

PLA 114 E

PLA 1143

PLA 1144

PLA 1145

PLA 1146

PLA 1147

PLA 1148

PLA 1149

PLA 1157

PLA IISI

PLA 1152

PLA 1153 


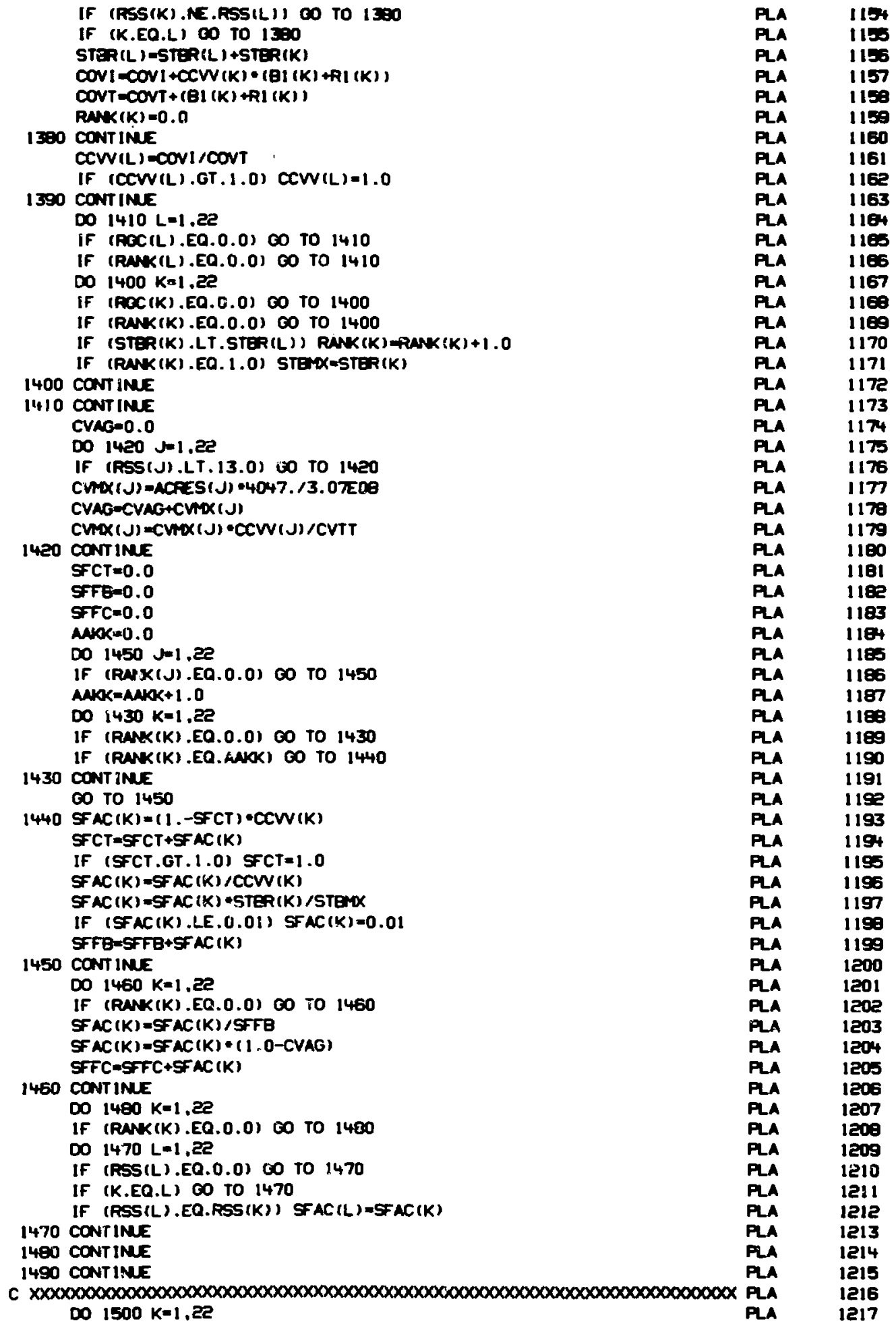


If (RAM(K).EQ.0.0) 00 to 1500

STER(K) =CVMX(K)

COV $(K)=A C R E S(K)$

PLA 1219

CVRX(K) $=$ SFAC (K)/SFFC

PLA 1220

1500 CONTIME

PLA IZ2!

$I I=I I+1$

PrINT 20s0, All

PLA

1 ट्2

PRINT zOso

PLA 1223

DO 1510 LPN-1, 22

PLA IE24

IF (RCCILP).EQ.0.0) CO TO 1510

PLA IEZS

PLA IEES

PRINT 2030, HI (LPN), RI (LPN), DI (LPN), HI (LPN), ROI (LPN), RHI (LPN), CCVV PLA

I (LPN), PI, TAY, HURAT (LPN), TPCNW(LPN), AI (LPN), LPN $\quad$ PLA IEC9

1510 CONT INUE

PRINT 2040

DO ISEO LPNTI, ES

IF inCC(LFN).EO.0.0) GO TO I5e0

PRINT 2030, A2(LPN), Z2L(LPN), Z3 (LPN), Z4 (LPN), Z4RILPN), Z5(LPN), Z5R

1 (LPN), Z6(LPN), Z7 (LPN), ZB(LPN), Z9(LPN), ZSR(LPN), LPN

15:0 CONTINUE

FRINT 2070

DO 1530 LPN=1, .2S

If (RCCLPN).EO.D.0) 60 TO 1530

Z7Pan.0

ZTFP=0.0

IF (DI (LPN).GT.0.0) ZTP=Z4 (LPN) /DI (LPN)

IF (FDI (LFN) .GT.0.0) ZTFR-ZUR(LFN)/FOI (LPN)

AEZ-AEILPNI /EP

A23-A2ล.1ZP

$Z 77=(Z 7$ (LPN) $+A Z$ (LPN) $/ B 1$ (LPN) ) /ZP

PRINT Z030, Z77, Z7P, Z7PR, X8(LPN), $A 22, A 23, Z 1$ (LPN, I), Z1 (LPN, 2$),$ CFAC

1 (LPN), SFAC (LPN), LPN

1530 CONTINLE

DO I540 LPNWI, 2e

If (ROCLILN).EQ.0.0) GO TO 1540

AMA (LFN) $=0.0$

IF (ROCLLN).NE.Z.O) GO TO 1540

CALL FORMN (II, LPN, Z9(LPN), MAA(LPNI)

FRINT 1660, RINGT (LPN)

1540 CONT INUE

1550 CONT INUE

co To 10

C

1560 FOFAT (EE 10.3$)$

1570 FORMAT (EF10.0)

IEO0 FOFMT (IHA,5OX, -TRU-BIOLOSICAL TRANSFORT MOOEL *)

PLA 1230

PLA 1231

PLA 1232

PLA 1233

PLA 1234

PLA 1235

PLA 1236

PLA 1237

PLA 1238

PLA 1239

FLA 1240

PLA 1241

PLA 1242

PLA 1243

PLA 1244

PLA 1245

PLA 1246

PLA 1247

PLA 1248

PLA 1249

PLA 1250

PLA 1251

PLA 1252

PLA 1253

PLA 1254

PLA 1255

PLA 1256

PLA 1257

PLA 1259

PLA 1259

PLA 1260

PLA 126I

FLA 126?

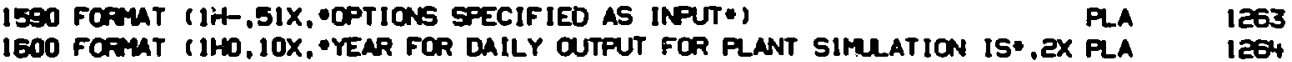

1.171

PLA IZES

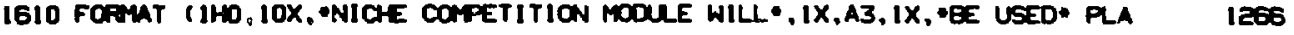

11

1620 FOFMT IID. IOX. "SUCCESSION MOOULE WILL", IX,A3, IX. "EE USEO" )

PLA 1267

PLA $\quad 1208$

1630 FOFMT (1HO, IOX, "CHFCNIC EVENT OPTION HILL", IX,A3,1X, "BE USED*)

1640 FOFMT (IHO, IOX. IFRIGATION WILL", IX,A3, IX, "EE USED*)

PLA $\quad 1200$

IESO FOFMT (IHD, 10X, "FOFMW SUEROUTINE HILL*.IX.A3, IX. "EE CALLD")

IGSO FOFMT (1OX, *RINGT INCREMENT, INCHES*,E11.4)

1670 FOFMT (1HO, 10X, "FORCUT SUBROUT INE HILL , $1 X, A 3,1 X$, "EE CALLED")

1600 FOFAT (1HD, 10X, "PONANT SUEROUTINE WILL , $1 X, A 3,1 X$, "EE CALLED*//1)

IESO FOFMT (IH-,47X, "TEMFERATURE CHARACTERISTICS FOR SITE*)

PLA 1270

PLA IETI

PLA IETE

FLA 1273

PLA 1274

1700 FOFMT (IHD,EX, MAX. TEMP. VARIATION,DEG. F " MIN. SOLAR RADIATI PLA

ION LY/DAY " MAX. SOLAR RADIATION LYIDAY " "RELATIVE ELEVATION, PLA

EFEET ABOVE SEA LEVEL 1

1710 FCFMT (IHD,TIS,E10.3,T48,E10.3,T78,E10.3.T119.E10.3)

PLA

1275

1270

1277

1278

1279

1720 FCFMT (1HD.ZX."HIN. MONTHYY TEMERATURE,DEG. F • MAX. MONTHY PLA 1280 ITEMPERATURE,DEg. F *LATITUDE RELATIVE TO SITE*)

PLA

1291 
1730 FOTMT (IHD. T23.E10.3.T50,E10.3, TEO,E10.3111)

1740 FOAMAT (IH-.40X. QPFECIPITATION CHAMCTERISTICS FOR SITE॰)

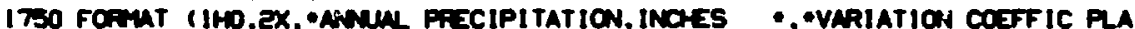

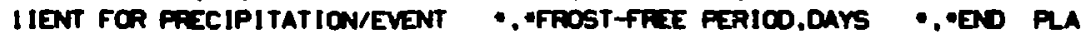
aFROST FREE PERICON)

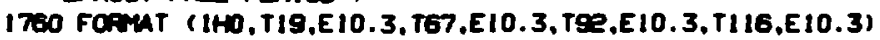

1770 FOFMA (IHD, IX, MONTHEY PRECIPITATIONS, FRACTIONE)

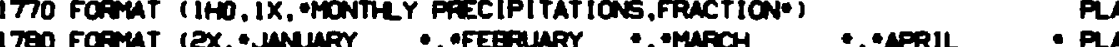

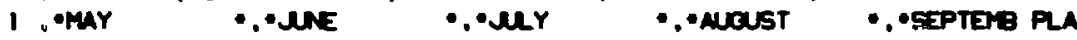

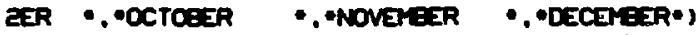

PLA

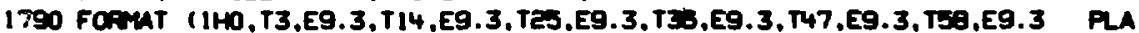

1 .T69.E9.3.TE0.E9.3.T91.E9.3.T102.E9.3.T113.E9.3.T124.E9.31 PLA

1800 FORMT (IHD, 1X, *TOTAL RAINEALL EVENTS/MONTHE)

1810 FORMAT (1HO, $17 x, E 10.3)$

PLA

1820 FORMAT (1H-,47X, "SOIL CHARACTERISTICS OF SITE*)

PLA

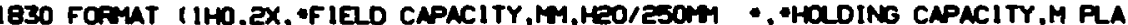

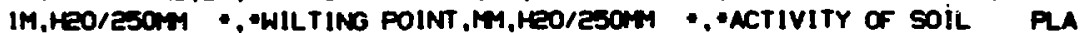
2•. -SOIL DEPTH,M⿻)

FLA

1840 FCRMAT (1HD,TI8,E10.3,T50,E10,3,T78,E10.3,T97,E10.3,TI14,E10.3/I/) PLA

1850 FOFMT (3X, $901 L$ ACTIVITY, ACT/CRAM DRY WEIGHT IN GIVEN SOIL HORIZ PLA $10 N(21) \cdot .3 X, "$ PLANT NO. $=-3 X, 14)$

1860 FORMT (3X. *ROOT FRACTION IN GIVEN SOIL HORIZON (ZF)

1870 FOFAT (3X. UPTAKE COEFFICIENT FOR A RADIONACIDE IN A GIVEN SOIL IHORIZON $(2 \mathrm{Z}) \cdot 1$

18E0 FOFMT (3x, 12E10.3)

IESO FCFAMT (IH-.44X. •CARONIC MD CONTAMINATING EVENTS")

PLA

PLA

PLA

PLA

PLA

1900 FOFMT (1HD,-INITIAL ACT. IN ATMOSPHERE/ME",EX."TOTAL " OF YEARS O PLA IFEVENT , 2X, •OCRATION OF EVENT,DAYS*, EX, -YR. OF INITIAL CONTAM. EVE PLA ENT".2X, "YR. OF CONTAM. ENDED")

1910 FOFMT (1HD, T19,E10,3, T46, E10.3, TE9, E10.3, T99, E10, 3,T119, E10.3)

I9EO FOFMT IIH, "DAY CF INITIAL CONTAM. EVENT:, 3X, DOAY OF FINAL CONTAM PLA I INATION*)

I930 FOFMT (IHO,T18,E:0.3,T47,E10.3?

I940 FOFMAT (IH. 47X, +IFPIGATION CHARACTERISTICS FOR SITE*)

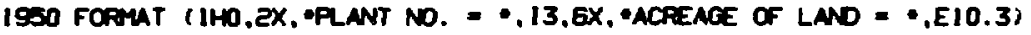

1950 FORMT (1+D,EX, *DAYS OF YEAR WATER APPLIED*)

1970 FORMAT (3X, 1EE8.2)

1980 FORMAT (IHO,2X, "WATER APPLIED ACRES/INCHES FOR DAYS OF YEAR")

1990 FCFMT (IHO, EX, "HARVSTING DATES, DAY OF YEAR*)

2000 FOFMAT (3X,EEI1.4)

2010 FORMAT (IH. "OAILY OUTPUT FOR YEAR •.E9.3)

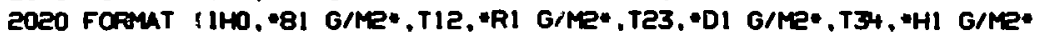
1 .T45. •RD1 G/ME*.T56, *AHI G/ME*,T67. *T,DEG.C*.T79, •P*, TE9, *AI ACT,

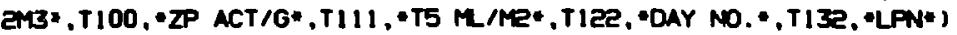

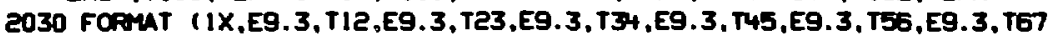
1 ,E9.3,T78,E9.3, T89,E9.3,T100,E9.3,TI11,E9.3,T122,E9.3,TI3e, I31

\section{PLA}

PLA

PLA

PLA

PLA

PLA

PLA

PLA

PLA

Pla

PLA

Pla

PLA

PLA

PLA

2040 FOFMT (1HD, "A2 ACT/ME",T12,"Z2 ACT/ME",T23, "Z3 ACT/ME*,T34, "Z4 AC PLA IT/ME", T45, "Z4R ACT/ME", T56,"Z5 ACT/ME", T67, "Z5R ACT/ME".T78, Z6 AC PLA 2T/G*,T89,*Z7 ACT/G*,T100,"Z8*,T111,•Z9 ACT/ME*,TIZ2,"ZGR ACT/ME* PLA 3 .T132, "LPN")

2050 FORMAT (1H-. -YEARLY OUTPUT FOR YEAR •.E9.3)

2060 FORMT (1HO, "BI G/ME",TI2, "RI G/ME*, T23, "DI G/ME*,T34, *H! G/ME* 1 .T45, *PD1 G/ME*, T56, *PHI G/ME*, TE7, "CCV*, T78, *P1 *, TE9, "TAV*

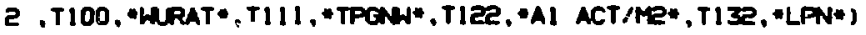

PLA

PLA

PLA

PLA

PLA

2070 FOPMAT (IHD, "Z77*. TIE, ZZP ACT/G*, T23, ZZFR ACT/G*, T34, "XB", T45, "A PLA 122 ACT/G*, T56, *A23*, T67, -21 (LPN, 1 ) *, T79, *Z1 (LPN, 2)*, TE9, *CFAC* $2, T 100$, SFAC*,T111, $L P N=1$

END

FUNCTION AIRAC (SACH, YR, DAY)

AIRAC=SACH

DETUFN

END

SURROUTIRE FOFMAN $(11, \mathrm{~K}, \mathrm{ZSO}, \mathrm{MA})$

COMON H1 (22), CCV $(22), A l(22), A 2(22), B l(22), R 1(22), 01(22)$, 


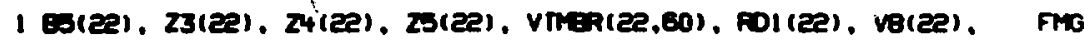

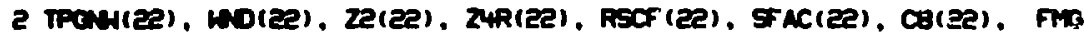

$321(22,32)$. CFAC(22)

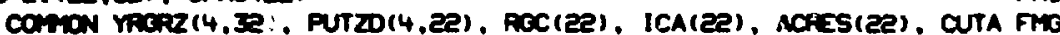

$1(20,8)$

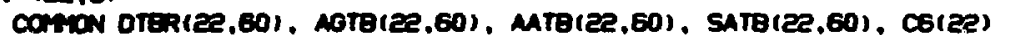

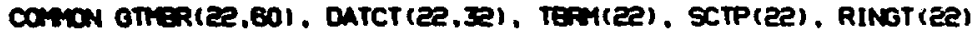

comon Z(EO). C7. Ba (60)

DIrENSION X(60), G(60), COV(60), BAR(60), hOT(60), XUI (2E), CO(E2)

DIMENSION COVSA(60), HOTSO160), BARSO(60), ZZ3(Z2)

DIMESSION MIND(Z2), TNERT (2E), WASTR(2E, 20). BEP(2E), VA(EO)

DATA MOT, ELAK, OLY, YFLY /3HOT.3H , GOAILY, GHEARYY'

If (TEPMINK).NE.0.1) CO TO 10

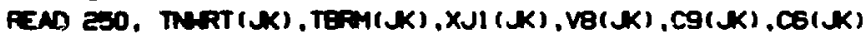

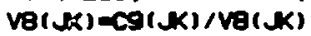

INERT = TNERT ( $K)$

IERM-TERM(K)

$\operatorname{EBP}(\boldsymbol{K})=0.0$

$\operatorname{MinO}(\mathcal{K})=0.0$

mo

$\operatorname{RINOT}(K)=0.0$

READ Z\$O, (WRSTR( $K, L), L=1$, INERT)

IF (TERM (K).GT.0.0) FEAD Zs0, (DATCT $(K, L), L=1$, IERM)

IF (TEPM (K).GT.0.0) CALL FORCUT $(11, M, Z 99, M, W$ )

FETUPN

10 continue

AlI-II

IERM-TEPM(K)

If (IERM.EO.O) IERMA

DO $20 \mathrm{~K}=1,1 \mathrm{EPM}$

If (TERAI (K).EQ.0.0) GO TO 20

If (DATCT $(K, K) . N E . A 11+1.01$ GO TO 20

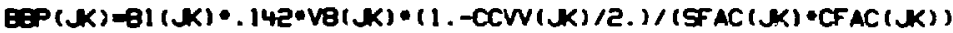
PETUR

20 CONTINUE

TCR APSCF (K)

If $(R O C(\sqrt{K}) . E Q .3 .0) \quad T C R=355$.

$T 1-773 . *(X) 1(K) / 2).+11 .-C C V W(J) / 2$.

$\sqrt{5}-.142 \cdot V B(K) \cdot B 1(K) \bullet(1,-C C W(K) / 2) /,(\operatorname{SFAC}(K) \cdot C F A C(K))$

$C B(K)=1.0$

IF (VS.LT.TI) RETUFN

$\operatorname{MIND}(K)=\operatorname{MIND}(K)+1.0$

G4-. 142*Bl (K) $\cdot V B(K) \cdot(1,-C C V V(K) / 2) /,(S F A C(K) * C F A C(K))$

O4-OH-EP(K)

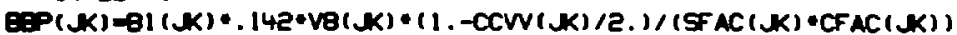

$\sqrt{4}=0.142 \cdot 81(\mathrm{~K})$

MAIND(JK)

If (M.GT.1) GO TO 40

DO $30 \mathrm{~L}=1,60$

$\operatorname{VTMER}(K, L)=0.0$

DTERI $K, L)=0.0$

$Z(L)=0.0$

GTMER $(K, L)=0.0$

$\operatorname{AGTB}(K, L)=0.0$

$\operatorname{MATB}(K, L)=0.0$

SATB $(K, L)=0.0$

$G A(L)=0.0$

$V A(L)=0.0$

$2 Z 3(\sqrt{K})=0.0$

30 CONTINUE

40 CONTINUE

$v 2=0.3 *(0.035 * v 8(K)) *(2.0 * 2.54) * * 2$

COTMG-VTMER(N, 1$)$

GTMER(NK. 1$)=T 1 * \operatorname{EXP}(-(1 . / R S C F(. X)) *(\sqrt{5}-T 1))$ 
If COTMER(JK, I1.GT.VS) GTMER(JK, 1) US

If (OTMER! K, I).EO.VS) FETUN

If (OTMER(NK.1).LE.VE) GTMER $, K, 1)=0.0$

VTMER(K, I) $=O M E R(K, 1) \cup V 4 / V 5$

DGTMEVTIER(N, 1)-GGTMB

DTER(K. I) DVTMER(JK. II/Ve

AOSUM $=0.0$

ACTSY $=0.0$

TREES $=0.0$

HTAV $=0.0$

COVAV=0.0

BMAV $=0.0$

HTSO $=0.0$

CVSO=0.0

$B S O=0.0$

$\operatorname{co}(\mathrm{K})=1.0$

C4=0.0

CVTR=0.0

00 BO $N=1,60$

ANAN

$\operatorname{COV}(N)=0.0$

BAR(N) $=0.0$

HGT $(N)=0.0$

$X(N)=0.0$

IF (GTMER(K.N).LE.0.0) CO TO 60

$M N=A N \cdot X J I(K)-X J I(K) / 2$.

$X(N)=(A N * C G(K)+Y B(K)) *=2$

$X(N)=X(N) \bullet(A N * \bullet) \bullet D T E R(K, N)$

If (N.EO. I) 60 TO 70

$\operatorname{COV}(N)=.0929 * 3.1416 *((A N+\operatorname{Co}(N K)+V B(K)) / 2.1 * \cdot 2$

HGT $(N)=(122 . /(V B(\mathrm{~K}))) *(A N / 24) *$.

$\operatorname{BAR}(N)=A N$

$\operatorname{COV}(N)=D T E R(K, N) \cdot \operatorname{COV}(N)$

HGT (N) =DTER (K,N) *HGT (N)

$\operatorname{QAR}(N)=\operatorname{OTER}(K, N) \bullet \mathcal{L C G}(B N R(N))$

TFEES= TREES+DTER $(K, N)$

$\operatorname{COVSO}(N)=\operatorname{DTER}(K, N) \bullet(\operatorname{CON}(N) / \operatorname{DTER}(K, N)) \bullet 2$

HGTSO (N) $=\operatorname{DTER}(K, N) \bullet(H G T(N) / O T E R(K, N))=2$

BNRSO(N) =OTER $(K, N) *(B A R(N) / D T E R(K, N)) \bullet \bullet$

IF (ARTB(NK,N).LE.0.0) G0 TO 50

AGSUM=AGSUM+DTER (K,N) * $L O G(A M B(K, N))$

50 IF (SATB(K,N).LE.0.0) CO TO 60

$A C T S Y=A C T S M+D T E R(K, N)=\mathcal{L C O G}(\operatorname{SATB}(K, N))$

60 HTAV-HTAV+HGT (N)

COVAV=COVAV+CON(N)

BAAV=BAAV+EAR(N)

HTSOHTSO+HGTSO(N)

CVSO-CVSO+COVSO(N)

BSO-BSO+EARSO(N)

HGT (N) =HGT (N) /DTER ( K.N)

$\operatorname{COV}(N)=\operatorname{COV}(N) / D T E R(N, N)$

BAR(N)=AN

CVTR=CVTR+DTER(K, N) *3. 1416*(BAR(N)/24. )・・2

70 IF $(X(N) . L E .0 .0)$ GO TO 80

$\operatorname{C\theta }(K)=\operatorname{C\theta }(N)+X(N)$

IF (N.GT.1) $C 4=C 4+1 / X(N)$

BC CONTINE

IF (TREES.LE.0.0) GO TO SO

DF = TREES- 1.0

IF (OF.LT.I.R) DF=1.0

BSD= (BSO-BAAV* $\bullet$ / TREES ) /OF

HTSD= (HTSO-HTAV • $2 /$ TREES)/OF

CVSD- (CVSO-COVAV *2/TREES ) /OF

BSD-EXP(BSD)

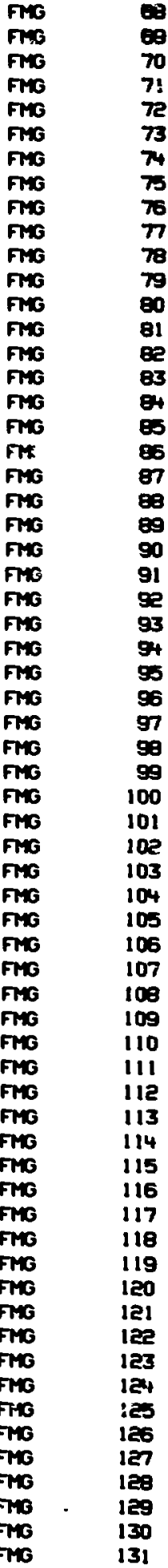




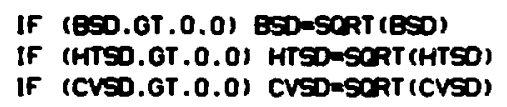

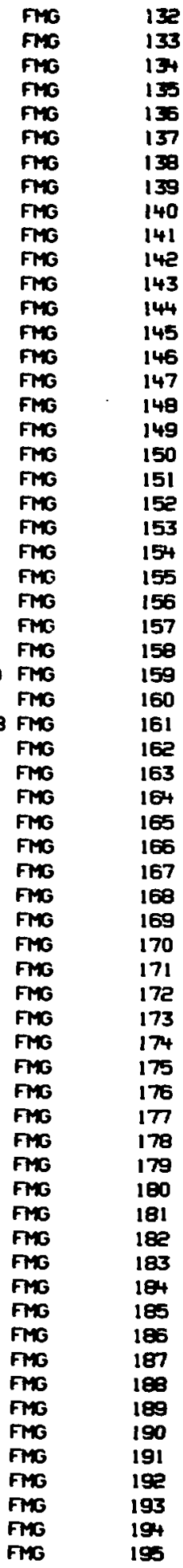


IF (WTM.LE.0.0) WTMEVTER(K.L)

IF (VTM.OT.VTER(K.LU) WTHOVTER(K.L)

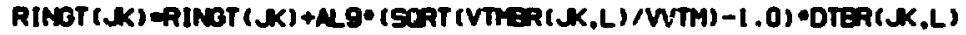

VA(LIOVTIER(K,L) ESATE(N,L)/0. L4?

$V A(L)=V A(L)+A B S(O 4) * A C T S * X(L) / C \theta(N)$

SATE(K,L) =VA(h)/(VTRE(K,L)/, 142)

VA(L) IVA L I + (NDTEN/ . 142) *SATE(K,L)

IF (VACLI.LT.0.0) YA(L)=0.0

IF $(X(L) . E Q .1 .0)$ SATE(KK,L)=SATE(K.L-1)

140 CONT INUE

IF (TRES.GT.0.0) RINGT (K) ARINGT (K)/TREES

$\operatorname{VA}(1)=V \operatorname{MBR}(\mathrm{K}, 1)=\operatorname{SATB}(\mathcal{K}, 1), 142$

$\operatorname{SATE}(\mathcal{K}, 1)=0.0$

IF (YMMR(K, l).LE.0.0) VA $(1)=0.0$

IF (VIMER(K.l).LE.0.0) CO TO 150

$V A(1)=V A(1)+1 B S(G 4) * A C T S=x(1) / C B(J)$

SATB(K, 1$)=V A(1) /(V T R E R(K, 1) / .142)$

VA $(1)=V A(1)+(D G T B / .142)$ SATE $(K, 1)$

IF (VACI).LT.0.0) VA(1)=0.0

SATE(K, 1$)=V A(1) /(V I N E R(K, 1) / .142)$

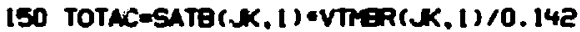

IF (TOTSA.EO. I.0) TOTAC=1.0

IF (TOTAC.LE.0.0) TOTAC $=1.0$

DO 180 LE=1,60

IF IAGTERM,LI.GT.0.01 GO TO I60

If (VTRER(K,L+1),GT.0.0) AGTE(K,L)=AII

I60 If IL.EQ.1) CO TO 180

If (AGTEIK,L-1).EQ.0.0) GO TO 170

IF (L.EQ.2) MATB(NK,L-I)=AGTB(KK,L-1)/2.0

IF (L.EO.e) GA(L-1)=AGTB(KK.L-I)

IF (L.EO.Z) GO TO 170

$A \operatorname{MTB}(\mathcal{K}, L-1)=(A G T B(\mathcal{K}, L)+A G T B(\mathcal{K}, L-1) / 2.0$

IF (AGTERK,L).EQ.0..AND.L.GT.Z) $M A T B(M K, L-1)=2 . * A A T B(J K . L-I)$

$G A(L-1)=A G T B(K . L)-A G T B(N, L-I)$

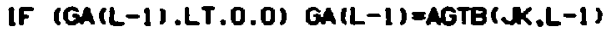

170 CONTINUE

IF (VTMER(K,L).LE.0.0) GO TO LEO

TOTAC=TOTAC+SATE $K, L, L)=V T R E R(K, L) / 0.142$

180 CONTINUE

IF (TOTAC.EQ. 1.0) TOTSA=1.0

SATE(K, 1)=SATB $(K, 1)$-TOTSA/TOTAC

IF (SATB $(K, 1) . L T .0 .0)$ SATE $(K .1)=0.0$

DO 190 LK=1. INHRT

IF (WRSTRIK.LK).EQ.A11) CO TO 200

190 CONTINUE

HRT $=0.0$

GO To 230

200 XNT $=1.0$

BAR( (1) =8AR( (1)/4047.

$\operatorname{COV}(1)=\operatorname{cov}(1) / 4047$.

IF (ROC (K).EQ.Z.O) PRINT Z70, YRLY.TNLRT (K)

IF (ROC(N).EQ.3.0) PRINT 270. DLY,TNURT(JK)

IF (TERM(N).GT.0.0) PRINT ZZO

IF (TEPM(NK).GT.0.0) PRINT 260, (DATCT $(K, L), L=1$, IERY)

PR!NT ZEO

KJ-JK

PRINT 310, HTAV, HTSD,CMN, CVSD, EN, BSD, COVAV, BATR, TREES, AGAVE

1 . ACTAV

Be= 1.0

PRINT ESO

$K J=J K$

PRINT 310, B2,VTMER(KJ, 1),DTER(KJ,1),TI,V4,SATE(KJ, 1),CVTR

D 210 L=2,60

사녀.

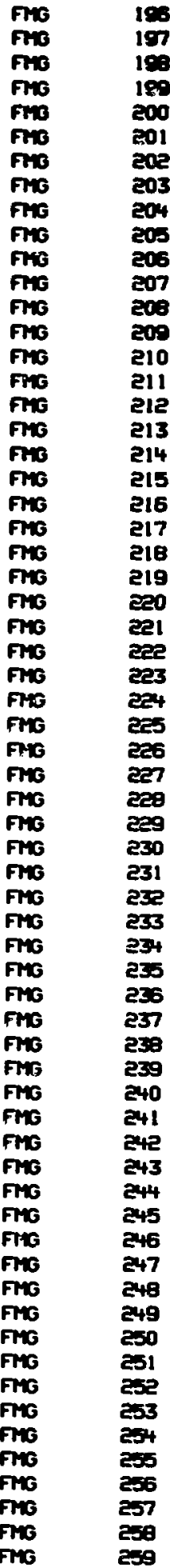




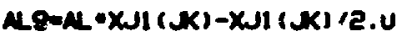

FTE

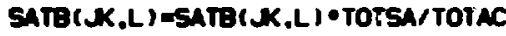

IF (SATB(K,L).LT.0.0) SATE( K,L) $=0.0$

210 contines

FRINT 500

Kulak

$\infty 200$ l-2.60

IF (OTHER(JK.L).EO.0.0) CO TO EEO

ALEL

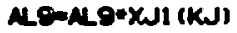

ALCALO-XUl (KJ),2.0

VOLTR-VTIER(KJ,L) /OTER(KJ,L)

FRIWT 310, ML, VTMER(KJ,L), GTHER(K! ,L), DTER(KJ,L), COV (L), BAR(L)

1 ,VOLTR,cA(L), MTtE(KJ,L), SATE(K,J,L)

2eo cont Ine

230 CONTINUE

$00240 \mathrm{~L}=1.60$

$Z 23(M)=Z 3(M)$

IF (XRT.EQ.0.0) SATE(M,L) =SATB(M,L) * TOTSA/TOTAC

240 CONTINE

IF (TERMIST).EQ.0.0) RETUPN

CALL FORCUT $(11, M, 299, M, M)$

C

RETURN

ESO FOMNAT (EF10.0)

FTE

FTE

FTE

FTE

FMG

FT:

FTrs

FT6

FT:

FTG

FTS

FT:

FIS

FYS

FTG

FIM

FTG

Fic

FME

FTS

FTS

FT:

FMG

FTS

280 FOKAT (3x,11E11.4)

FTG

270 FOFMT IIH-, "FOFMAN OUTPUT ON*,1X,A6."BASIS*,3X,F4,0.1X. "PRINTA,TS FTG

1 RECUIFEO I

FTM

EEO FOFAT (1H0, "HTAV*, T14, "HTSD", TE6, "CVMN, T38, "CVSO•, T50, "EN", TE2, FTG

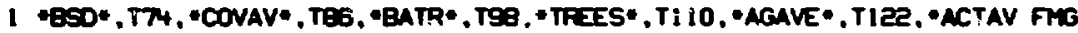
2*)

TMG

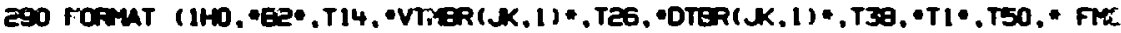
IV4*, T62."SATB(K, 1)", T74. "CVTR*)

FMG

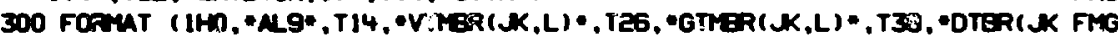

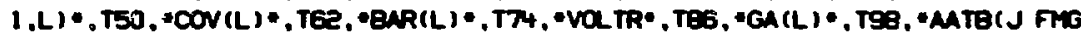

ᄅ K.L)*,T110, "SATE(K,L)*)

FTM

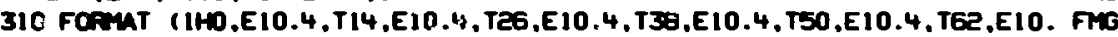

1 4.T74.E10.4.TES.E10.4,TS8,E10.4,T110.E10.4,T122.E10.41

FMS

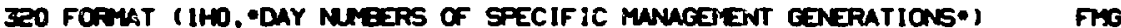

ED

SUEROUT INE FOROUT (II, M, 299, M, K)

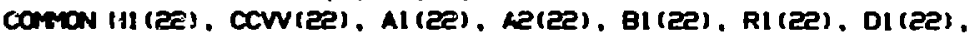

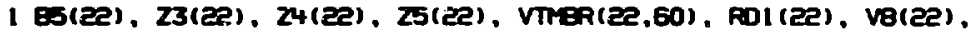

FMG

FCT

FCT

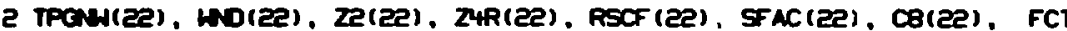

3 Zl(2e, 32), Graclez)

FCT

COMAON YRGRZ(4,32), PUTZO(4,Z2), RGC(22), ICA(22), ACFES(22), CUTA FCT

$1(22,8)$

COMNON DTER(22,60), AGTB(22,60), MATB(22,60), SATB(22,60), CS(22) CONAN GTIER(22,60), DATCT (22,32). TBPH(22), SCTP(22), RINGT (22) COMneN Z(60), C7, GA 60 )

FCT

DIrension tCUT (2e,32), ACUT $(22,2)$, BCUT $(22,2), \operatorname{COUT}(22,2)$, VCUT (60 FCT

1 I

DIMENSION RACUT (2e), RECUT (2e), RCCUT (2e)

IF (M.GT.O) CO TO 10

IERA=TEFM( $\mathbf{K})$

READ 190, (TCUT ( $\mathcal{K}, I), I=1$, IEFM)

FCT

FCT

FCT

FCT

FCT

FCT

FCT

C . 1-CHDICE CUT, SAWT IMEER (12-40), PULWOOD(6-10), PRECOMM(0-4), CLEAR CU ZMREVERSE-J CUT. AND NLL SIZES ABOVE Z6 INCHES DIA. REMOVED

FCT

FCT

3-FIRE OCCURANCE. ALL CLASSES AFFECTED

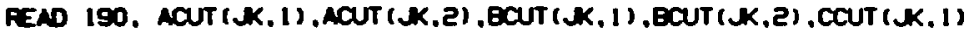

1 . Cout $(K, 2)$

RENO 190, RACUT ( $(K)$, RECUT ( $M$ ) , RCCUT (J)

10 CONT INUE

IERT=TERM(K) 


\begin{tabular}{|c|c|c|c|}
\hline & $A 11=11$ & FCT & 26 \\
\hline & $\begin{array}{l}D 020 \quad K=1 \text {, IEFY } \\
\text { KK=K }\end{array}$ & $\begin{array}{l}\text { FCT } \\
\text { FCT }\end{array}$ & $\begin{array}{l}27 \\
29\end{array}$ \\
\hline & If (OATCTIN.K).EO.All) 60 To 30 & FCt & 29 \\
\hline 20 & Cont Inve & FCT & 30 \\
\hline & RETURN & FCT & 31 \\
\hline 30 & ITC-TCUT $(\boldsymbol{K}, \mathrm{NOK})$ & FCT & 32 \\
\hline & vrot $=0.0$ & FCT & 33 \\
\hline & $\infty 040 k=1.60$ & FCT & 34 \\
\hline 40 & $\operatorname{vart}(K)=0.0$ & FCT & $\mathbf{3 0}$ \\
\hline & IF(ITC.EO.E)GOTOIZO & FCT & 36 \\
\hline 50 & $|A|=A C U T(\boldsymbol{K} .1)$ & FCT & 37 \\
\hline & IA2-ACUT (K. (K) & FCT & 38 \\
\hline & IBI -ECUT (KK, 1) & FCT & 39 \\
\hline & 182-ecur (JK,2) & FCT & 40 \\
\hline & ICI-COUT $(\boldsymbol{K}, 1)$ & FCT & 41 \\
\hline & ICE-cout $(\pi, 2)$ & FCT & 42 \\
\hline & If $(|A| . E O .0) 00$ TO 70 & FCT & 43 \\
\hline & $D 060 \mathrm{~K}=|A|, \mid A 2$ & FCT & 44 \\
\hline & IF (VTMEA(JK.K).LE.0.0) GO TO 60 & FCT & 45 \\
\hline & $\operatorname{VCUT}(K)=\operatorname{VTRER}(K, K)=(1,-\operatorname{RACUT}(K))$ & FCT & 46 \\
\hline & $\operatorname{DTER}(K, K)=\operatorname{DTER}(K, K)=(\operatorname{VTRER}(\mathcal{K}, K)-\operatorname{VaVT}(K)) / \operatorname{VTMER}(K, K)$ & FCT & 47 \\
\hline & VTOT=VTOT+VCUT (K) & FCT & 48 \\
\hline 60 & CONTINEE & FCT & 49 \\
\hline 70 & IF $(181 . E 0.0)$ CO TO 90 & FCT & 50 \\
\hline & $D 00 \mathrm{~K}=1 \mathrm{BI} .1 \mathrm{1B2}$ & FCT & 51 \\
\hline & IF (VTKER(SK,K).LE.0.0) CO TO 80 & FCT & 52 \\
\hline & $\operatorname{vaUT}(K)=V \operatorname{MER}(K, K) *(1$, RaCUT $(K))$ & FCT & 53 \\
\hline & $\operatorname{DTER}(\mathcal{K}, K)=\operatorname{DTER}(K, K)$ ( VTRER $(K, K)-\operatorname{VCUT}(K)) / \mathrm{VTRER}(\boldsymbol{N}, K)$ & FCt & 54 \\
\hline & VTOT=VTOT+VCUT (K) & FCT & 55 \\
\hline 80 & CONT INEE & FCT & 56 \\
\hline 90 & If (ICI.EO.0) GO TO 110 & FCT & 57 \\
\hline & D $100 \mathrm{~K}=1 \mathrm{Cl} . \mathrm{IC2}$ & FCT & 5 \\
\hline & IF (VTMER(K.K).LE.0.0) GO TO 100 & FCT & 59 \\
\hline & $\operatorname{VCUT}(K)=\operatorname{VTER}(K, K) \cdot(1,-\operatorname{RCCUT}(K))$ & FCT & 60 \\
\hline & $\operatorname{DTER}(K, K) \Rightarrow \operatorname{DTER}(K, K)+(\operatorname{VTRER}(K, K)-\operatorname{VCUT}(K)) / \operatorname{VTER}(K, K)$ & FCT & 61 \\
\hline & VTOT-VTOT+VCUT (K) & FCT & 62 \\
\hline 100 & CONTINEE & FCT & 63 \\
\hline 110 & IF (ITC.EQ.3) CO TO 150 & FCT & 64 \\
\hline & 60 то 170 & FCT & 65 \\
\hline 120 & DO $140 \mathrm{k}=1.60$ & FCT & 66 \\
\hline & $A K=2 .-K$ & FCT & 67 \\
\hline & If (VTMER(KK,K).LE.0.0) 60 TO 140 & FCT & 68 \\
\hline & IF (AK.GE.Z6.) CO TO 130 & FCT & $\boldsymbol{\infty}$ \\
\hline & $R V J=126.0 * E X P(-.197 \cdot A K)$ & FCT & 70 \\
\hline & RVJ-RVJ*Z(K) & FCT & 71 \\
\hline & If (VTRER(KK.K).LE.RVJ) CO TO 140 & FCT & 72 \\
\hline & VCUT $(K)=V T E R(K, K)-R V J$ & FCT & 73 \\
\hline & $\operatorname{DTER}(\mathcal{K}, K)=\operatorname{DTER}(K, K) *(\operatorname{VTRER}(\mathcal{K}, K)-\operatorname{VaUT}(K)) / \operatorname{VTEEA}(\mathcal{K}, K)$ & FCT & 74 \\
\hline & VTOT=VTOT+VCUT (K) & FCT & 75 \\
\hline & 60 TO 140 & FCT & 76 \\
\hline 130 & VCUT $(K)=V \operatorname{THER}(K, K)$ & FCT & $\eta$ \\
\hline & $\operatorname{DTER}(K, K)=\operatorname{DTER}(K, K)=(\operatorname{VTRER}(K, K)-\operatorname{VCUT}(K)) / \operatorname{VTMR}(\mathcal{K}, K)$ & FCT & 78 \\
\hline & VTOT=VTOT+VCUT (K) & FCT & 79 \\
\hline 140 & CONTINAE & FCT & 80 \\
\hline & 60 To 170 & FCT & 81 \\
\hline 150 & $160 \quad 160 \quad k=2.60$ & FCT & $\mathbf{8 2}$ \\
\hline & IF (VTMER(KK,K).LE.0.0.OR.VCUT (K).LE.0.0) CO TO 160 & FCT & 83 \\
\hline & $\operatorname{VTREA}(K, K)-V T M E R(K, K)-V C U T$ iK) & FCT & B4 \\
\hline & 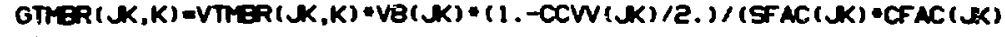 & FCT & Es \\
\hline & 11 & FCT & $6 \mathbf{6}$ \\
\hline & Z99-Z99+.7*VCUT $(K)=S A T B(K, K) / .141$ & FCT & 87 \\
\hline & $M A=M+0.1 * \operatorname{VaUT}(K) * \operatorname{SATB}(K, K) /(.141 * 30)$. & FCT & $\mathbf{8 8}$ \\
\hline & $2+(K)=24(\Omega K)+0.2 * \operatorname{SATB}(K, K) * \operatorname{VCUT}(K) / .141$ & FCT & eg \\
\hline
\end{tabular}


$23(\sqrt{x})-23(\mathrm{~K})-\mathrm{SATB}(\mathrm{K}, \mathrm{K}) \cdot \mathrm{vat}(\mathrm{K}) / .141$

ied contine

RDI (N) -FOI (NK) +RI (NK) *VTOT/C7

$Z 4 A(N)=Z 4 R(N K)+Z 2(N)$ VTOT/C7

$2 e(K)=Z 2(K)-Z 2(N) \bullet v T o t / C 7$

HND $(K)$ AND $(\mathrm{K}) \cdot(C 7-V T O T) / C 7$

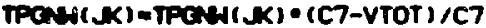

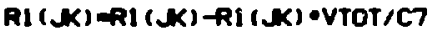

Bi (K) $-91(K) \cdot(C 7-V T O T) / C 7$

PRINT 210

FRINT 200, IERM

PRINT EEO, (TOUT $(S K, 1), I=1$, IERM)

PRINT 230

KJ=JK

PRINT 240, C7.VTOT.BI (KJ),Rl (KJ), DI (KJ), Z3(KJ), Z2(KJ).Z4(KJ), All RETURN

170 DI (N)

RDI (JK) + FDI (JK) +R] ( KK) *VTOT/C7

Do $180 \mathrm{~K}=\mathrm{e}, 60$

if (VTMER(JK,K).LE.0.0.0P.VOUT(K).LE.0.0) CO TO IBO

$\operatorname{VTER}(J K, K)=V \operatorname{TMER}(K, K)-V C U T(K)$

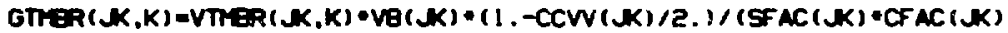
11

$24(K)=24(K)+.25 \cdot 5 A T \theta(N K, K)=\operatorname{VaU}(K) / .141$

$23(\mathcal{K})-23(\mathrm{~K})$-SATE( $K, K) \cdot V C U T(K) / .141$

180 CONT INUE

$Z 4 R(J K)=Z 4 R(K)+Z 2 E(K) * V T O T / C 7$

$Z 2(N)=Z 2(N)-Z z(N) \cdot V T O T / C 7$

LAD $(\mathrm{K})$ thD $(\mathrm{K}) \cdot(C 7-V T O T$ i /C7

TPCNA $(K)=\operatorname{TPCNA}(K) \cdot(C 7-V T O T) / C 7$

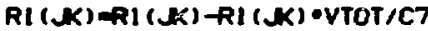

Bl (K)-B1 (K) • (C7-VTOT) /C7

PRINT 230

Kalk

PRINT 240, C7,VTOT, 81 (KJ), RI (KJ), DI (KJ), Z3(KJ), Z2 (KJ), Z4(KJ), AII FETURN

C

190 FOFMAT (EFID.0)

EOO FORAAT (IH,. •FORCUT OUTPUT TEPA *.17)

210 FOFMT IIHD.EX. *TYFE OF CUTTING EVENT, 1 = CHOICE CUT, 2 = FEVERSE

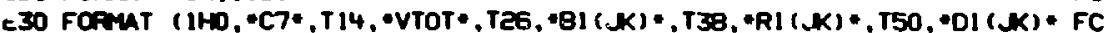

$1, T E 2, \bullet 23(\mathrm{~K}) \bullet, T 74, \bullet 24(\mathrm{~K}) \bullet, T E 5, * A 11 *$,$) FCT$

Z40 FOFMT (IHD,E10.4,T14,E10.4, T26,E10,4,T38,E10.4, T50,E10.4, TE2,EIO. FCT

$14, T 74, E ! 0.4, T E S, E 10.4, T S B, E 10.4)$

ENO

SUEROUT INE RANANT $(11,111$, AITT, XEERB)

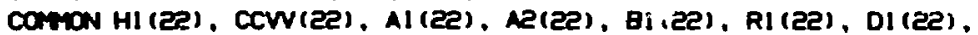

$1 E S(22) .23(22)$. $24(22), 25(22)$, VMER(22,60), RDi (22), VB(22),

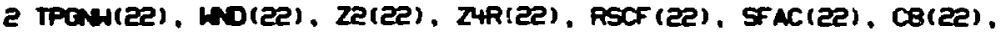

$321(22,32)$. CFAC(22)

COMTON MRERZ(4,32), PUTZO(4,22), RGC(22), ICA(22), ACRES(2e), CUTA AN

$1(2 e, 8)$

COMTON GTMER(22,60), DATCT(22,32), TERM(22), SCTP(22), RINGT(22) AN

Dirension $H(20,4), R(8,4), S(8,4), U(8,4), P(8,4), M(8,4), X(8,4)$. An

I $Y(8.4)$

DIMENSION ONFNO(4,32), FSUPF (4), RR(4), P4(22), PPG(22), CNIT(22),

I UP(3), Z(8,4), O(8,4), V(8,4), N5(4), P5(4), MAZ(4), PNIT(22).

a SNIT(Ee)

DIMENSION WATER(22), A(22), CSMM(3), GTDR(3), TNIT(3), SACT (3), I OFRAC (3)

DIMJNSION SAIR(3), MMAL (4,32), HMUS(4), G(22), D(22)

DIMENSION HFVST (4), HAVSP(4), SPAC (4), SPAS(4), SPAP(4), SPMA(4)

FCT

ANL

ANL

ANe

AN

an

ANL

MNL

ANL 
DITENSION SPAR(4), SAM $(4)$, TOTSF $(4)$, SPUT $(4)$

AN

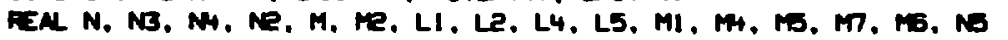
DATA IIe $/ 0 /$

AII $|=| I$

411 I $\mid$ I I

$115=\mid 12+1$

If $(112.6 T .1)$ co to 130

FEND 400, (FR(L), L- $-1,4)$

$0010 \mathrm{~J}=1.4$

Ps (J) $=0.0$

No(J) $=0.0$

$\operatorname{Mr}(J)=0.0$

FSUFF (J)=0.0

$\operatorname{SPUT}(J)=0.0$

IF (RA:J).EO.0.0) CO TO 10

PS-FRAC EEEF FFOO CONS IN REG, NOHO. ANIMLLS, MAZ-FRAC AREA CRUZED

FEAD 400, PS (J), NO(J), ME (J), FSUFF (J)

FEND 400, (MRCRZ (J.L),L=1,32)

REAO 400, (ONFNB(J,L),L-1, 3e)

READ 400, (ANAL $(J, L), L=1,32)$

10 CONTIREE

READ 400, (PUTZO $(J, L), L=1,22)$

Ne=1.0

$N+1.0$

$\mathrm{MI}=0.52$

$F I=0.04$

E6 $=0.25$

MB=0.6

$\omega(17,1)=550.0$

M7=3.73E08

W $(1,1)=23$.

H(2. 1$)=290.4$

$W(3,1)=410.3$

$\omega(4.1)=494.4$

$\omega(5,1)=550.0$

H(6, 1$)=500$.

$\omega(7,1)=50$.

$H(B, 1)=550$.

$z(1,1)=0.4$

$z(2,1)=.06$

$z(3,1)=.12$

$z(4.1)=.18$

$Z(5,1)=.12$

$Z(6.1)=.06$

$Z(7.1)=.035$

$z(8,1)=.024$

$\omega(11,1)=0.0$

$Z(1,3)=0.25$

$Z(2,3)=0.23$

$z(3,3)=0.15$

$Z(4,3)=0.12$

$2(5,3)=0.12$

$z(6,3)=0.05$

$2(7.3)=0.05$

$2(8,3)=0.02$

$\omega(11.3)=0.0$

c

L=ANIMLL TYPE, KAPOPLATION CATEGORIES

DO $30 L=1.4$

HFVSP $(L)=1$.

HRVST $(L)=1$.

$\operatorname{SPAC}(L)=0$.

$\operatorname{SPAS}(L)=0$.

$\operatorname{SPAP}(L)=0$.

$\operatorname{SPAR}(L)=0$. 
$\operatorname{SPAA}(L)=0$.

$\operatorname{SAP}(L)=0$.

HrISIL $8=0$.

TOTSF $(L)=0$.

If (FACL).EO.0.0) co TO 30

$0020 \mathrm{Kel} . \mathrm{O}$

$Z(K, L)=Z(K, I)$

IF (L.GT.Z) Z(K,L) Z(K, 3)

C HI17, IN)MMTURE HT. OF HaREIVORE (KO.)

HI 17.L) MFA(L) H(17.1)

C H( K,IN)=CATECORY HT. COMPARED TO EEEF CATTLE, (KG.)

H(K,L ) ARR(L) *H(K, 1$)$

C M( K, IN) MVTAEOL IC LEIOHT (KG.)

$M(K, L)=(R R(L) * W(K, 1)) * 0.75$

C R( K. IN) ERINZN CAPACITY IKG.I

$R(K, L)=0,03 \oplus M(K, L)$

C S( K, IN) GIVIN FILL (KG/DAY)

$S(K, L)=R(K, L)$

C U( K, IN) MICEST IELE ORY MATTER (KG/OAY)

$U(K, L)=R(K, L)$

C P( K, IN) AHICROFFOTEIN LEVEL IN RUREN FER-GROP, GRAMS P(K,L)=Z00. OPRR(L)

20 CONTINE

30 CONTINEE
$0040 \quad I=1,8$
DO $40 \mathrm{~J}=1.4$
$0(1, J)=0$
$v(1, J)=0$

40 CONTIMEE

$0060 \mathrm{~J}=1,4$

IF (RR(J).EO.0.0) CO TO 60

$0050 \mathrm{~L}=1,22$

$\operatorname{SNIT}(L)=0.0$

IF (REC(L).EO.0.0.OR.PUTZOIJ,L).EQ.0.0) CO TO 50

PNIT (L)ARE

S्ञIT (L) $=$ N4

CNIT (L) $=5 N I T(L)$

$P P B(L)=1.0$

50 CONTINE

60 Cont InLe

Do $120 \mathrm{Jm} 1,4$

IF (RR(J).EO.0.0) CO TO 120

Do $110 \mathrm{~K}=1,22$

IF (RKC(K),EO.O.O.CR.FUTZO J,K) .EO.0.0) CO TO 110

FPG $(K)=0.0$

IPUTZ-PUTZO(J,K)

CO TO $(70,80,90,100)$. IFUTz

70 SPUT $(1)=$ SPUT $(1)+1.0$

co to 100

80 SPUT (2) $=$ SPUT $(2)+1.0$

co to 100

SO SPUT (3)-SPUT $(3)+1.0$

100 CONTINE

110 CONTINE

1 IE CONTINE

130 CONTINE

IF (11.EO.O) RETURN

Do $160 \mathrm{~L}=1.22$

$A(L)=0.0$

$G(L)=0.0$

$D(L)=0.0$

$P+(L)=0.0$

IF (FGC(L).EO.0.0) CO TO 160

IF (SNITIL).EQ.0.0) CO TO 160

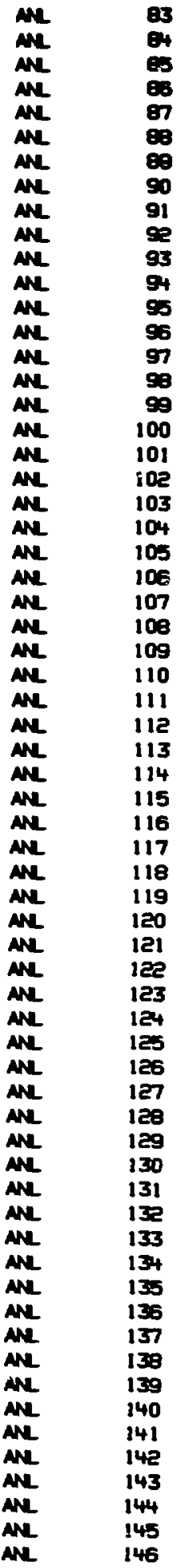




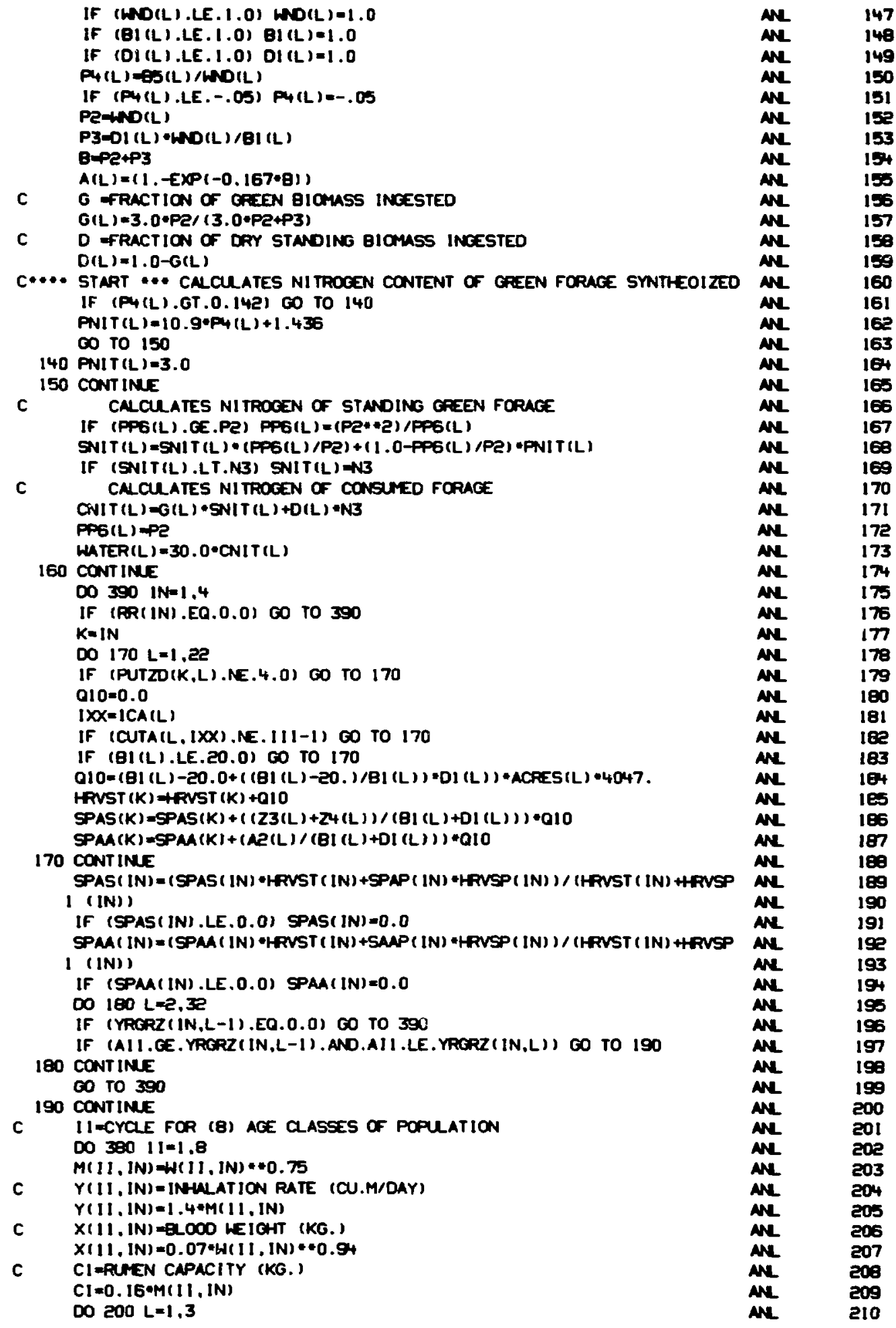


CSMM(L) $=0.0$

GTRRILI=0.0

NITILI $=0.0$

SACT $L L=0.0$

SAIRIL $1=0.0$

CFRAC (L) $=0.0$

200 cantine

Oxl-S(II, (N)

If (0.LE.0.001) $0=0.001$

DO $220 \mathrm{~L}=1,22$

If (PUTZO IN.L).EO.0.0) CO TO ZZO

KAPUTZO IN,L)

CO TO (210,210,210,220), K

210 CONT INE

Q10 $($ KD $(L) \bullet G(L)+D(L) \bullet D I(L) * W N D(L) / B 1(L)) * A(L)$

CSUM(K)-astm(K)+Q10

GTOR(K) $=$ GTDR $(K)+G(L) \bullet W N D(L) * A(L)$

TNIT $(K)=$ TNIT $(K)+C N I T(L)=Q 10$

$S 10=(W N D(L)=G(L)+23(L)+D(L)=D 1(L) * W N D(L) * Z 4(L) / B 1(L))=A(L) / Q 10$

$\operatorname{SACT}(K)=\operatorname{SACT}(K)+S 10$

IF (SACT (K) .LE, 0.0) SACT $(K)=0.0$

$S A O=(W N D(L) * G(L) * A 2(L)+D(L) * D I(L) * W N D(L) * A Z(L) / B I(L)) * A(L) / Q 10$ SAIR(K) $=$ SAIR(K)+SAO

IF (SAIR(K).LE.0.0) SAIR(K)=0.0

2EO CONTINUE

$\operatorname{CFRAC}(1)=1.0$

If (CSUM(e).EO.0.0) CO TO 230

$\operatorname{CFRAC}(1)=.78$

CPAC (2) $=.22$

IF (Csum(3).EO.0.0) GO TO 230

CFrac (1) $=0.7$

$\operatorname{CrRAC}(2)=0.20$

$\operatorname{OFRAC}(3)=0.10$

HATCAV=0.0

$A=0.0$

230 DO $240 \mathrm{~K}=1.3$

IF (CFRAC (K) . GT . 0.0)AU=AN+1 . 0

WFAC $=(1.0-0.3 *$ NNI T (K) /OSUM(K) )

UP(K) EFRAC (K) *O*WFRAC

HATCAVEUTCAV+WATFRAC

240 CONT INUE

C 0 -COMPUTES INCESTION RATE (KG/DAY)

$0=(1)(1)+U P(2)+U P(3)$

$N=0.0$

GA=0.0

$\operatorname{SPAC}($ IN $)=0.0$

SPAR(IN) $=0.0$

DO $250 K=1.3$

If $\operatorname{COSUM}(K) . L E .0 .0)$ GO TO 250

SPAC (IN) =SPAC (IN) +UP (K) SACT (K) / (CSMM $(K)=0)$

SPAR (IN) =SPAR (IN) + HP (K) -SA IR $(K) /(\operatorname{CSAM}(K) * 0)$

$N-N+U P(K) *$ TNIT (K) / (OSUM $(K) \cdot 0)$

GA-GA+UP (K) -GTOR (K)/ (OSUN $(K) * 0)$

250 CONT INUE

IF (II.GT.I) CO TO 260

C N AREADUSTS NITROGEN CONTENT TO ACCONT FOR INGESTION OF MILK $N=3$

260 CONTINE

$K=1 \mathrm{~N}$

DO 270 L-e.32

IF IAIII.GE.ONPAG(K,L-1).AND.AIII,LE.ONFNG(K,L)) CO TO ZEO

270 CONTINE

CO TO 380

zeO CONT INUE

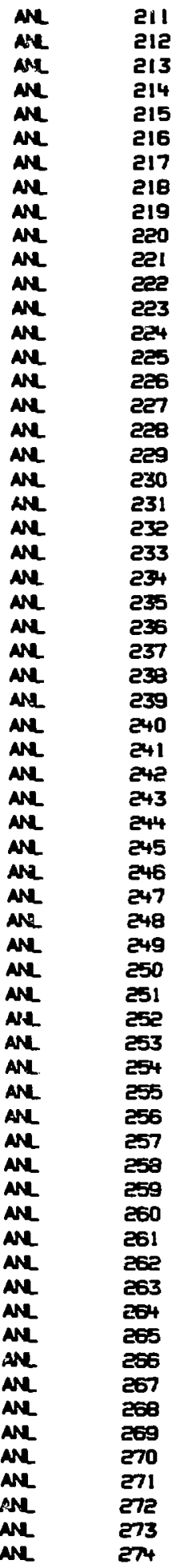




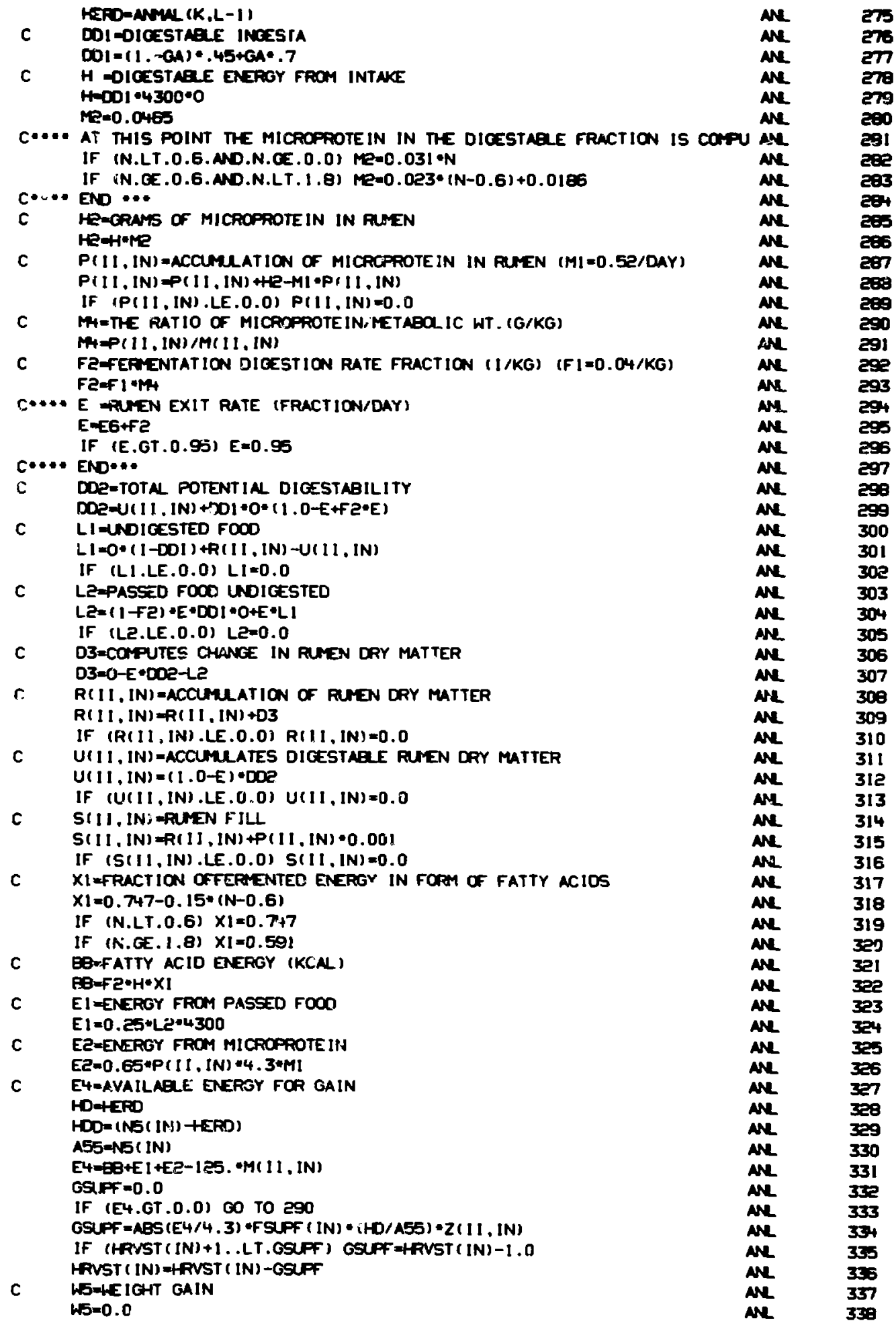


IF (FSUPF (IN) .EQ.0.0) W5-E4/1700.

CO TO 310

esO continte

C HB-RATIO OF HEIGHTMATUFE HEIGT

HB-W(J), IN)/W(I7. IN)

If (WB.CE.1.0) CO TO 300

C ESHEICHT GAIN AOUSTMENT FACTOR

ES=3.74+10.52*h3

IF (WB.LT.0.75) ES $=3.74+5.26 \cdot W 3$

IF (HB.LT.0.5) ES=1.5

LS-EY/ (ES-1700.)

CO TO 310

$300 \mathrm{HS}=0.0$

310 CONTINUE

$W(I 1$, IN) WW (II. IN) the

C GI -CPAMS OF GREEN FORAGE CONSUMED

O1-GA=0.1000.

C DH-CPANS OF DPY FORACE CONSUMED

DH= $(1,-$ GA $)=0 \cdot 1000$.

C CE-TOTAL CRANS OF FORAGE CONSUMED C2-GI+DH

IF (CE.LE.0.0) CZ=0.0

C C3=TOTAL PU INCESTED

C3=(HD/ASSS) *C2* (SPAC (IN)+SPAR(IN))

C3-C3+CSOPF * (SPAS ( IN) +SPMAI IN I)

TOTSF ( IN) - TOTSF ( IN) + GSUPF

GSUFF-125. - M (II, IN) *FSUFF ( IN *HDD*Z(II, IN)

IF (HPVST (IN) +1..LT.OSTPF) GSUFF+RVST (IN) -1.0

HFVST ( IN) +RVST ( IN) -GSUPF

C3-C3+CSUPF * (SPAS (IN) + SPMA (IN) )

TOTSF ( IN) - TOTSF ( IN ) + GSPPF

C C4=SQ.METERS CRAZED/ANIMAL UNITS

C4=Cz/ (COSM (1) +CSSM (2) + CSSM(3))

C MJ-SO.METERS GRAZED BY CATEGORY

MS-C4-Z (II I IN) HEFO

C MO-FRACTION OF AREA GRAZED

$M G+D \cdot Z(11,1 N) /(M 2(I N) \cdot M)$

$J=I N$

DO $330 \mathrm{~L}=1,22$

IF IFUTZO(J,L\},EO.0.0) 60 to 330

$K=P U T Z D(J, L)$

CO TO $(320,320,320,330), K$

$>20$ CONTINUE

$O 1 O=(W D(L)+G(L)+D(L)+O I(L)+W O(L) / B I(L))=A(L)$

CGO-G1 + $(010 / C S M M(K))+(L P(K) / 0)+M G$

CDO-DH+ $(010 / C S M M(K))+(L P(K) / 0)+M 6$

$23(L)=23(L)-23(L)+(000 / 81(L))$

$24(L)=27(L)-24(L)+(000 / D)(L))$

$M E(L)=-M E(L)-A 2(L)+(O C O+C D O) /(B)(L)+O)(L))$

BI (L) $-B 1(L)-C, 00$

$D 1(L)=D 1(L)-C D 0$

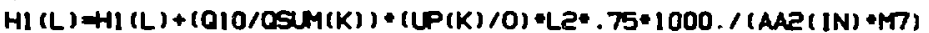

ane

ANL 340

AN 341

ANL 342

ML 343

AN 344

Ane 345

ANL 346

AN 347

AN $3+8$

ANL 349

ANL 350

ML 51

AN 352

AN 353

ANL 354

AN 355

ANL 356

MN $\quad 357$

ANL $\quad 358$

AN $\quad 359$

ML 360

AN 361

MN 362

AN 363

ANL 364

ANL 365

ML 356

AN 367

AN 368

AN 369

ML $\quad 370$

AN 371

AN 372

Ne $\quad 373$

AN 374

Ane 375

Ane 376

Ane 377

ANe 378

NL 379

ANe 380

AN 381

ANe 382

ANe 383

aNe 384

AN 385

AN 386

AN 397

AN 389

ANL 389

AnL 390

If $(Z 3(L) . L T .0) \quad Z .3(L)=0$.

If $(Z 4(L) . L T .0) \quad Z+.(L)=0$.

IF $(A Z(L) . L T .0) \quad A Z.(L)=0$.

IF $(\theta 1(L), L T .1) B,(L)=1$.

IF $(D \mid(L) . L T .1$.$) DI (L)=1$.

$Z 5(L)=25(L)+$ HMS $(1 N) \cdot(010 / \operatorname{csum}(K)) \cdot(L P(K) / 0)$

330 CONTINUE

HeNS (IN) $=0.0$

CE=0.0

DO $340 \mathrm{~K}=1$, 22

If (ROC $(K), E 0,0.0)$ CO TO 340

IF (SCTP(K).EO.0.0) CO TO 340

ANL 391

AN 395

AN 393

AN 394

AN 395

AN 395

AN 397

NL $\quad 390$

ANL 309

AN $\quad 400$

ANe 401

AN 402 
If (PUTZO IN, K).EO.0.0) CO To 340

IF (PUTZO(IN,K).EO,4.0) CO TO 340

IFUTZ=FUTZD(IN.JK:

TSUPB-81 (NK)+DI (N)

FDIET LP ( IPUTZ) /SPUT ( IPUTZ)

C CS=CRAMS SOIL INCESTED/OAY

CS-3. *W(II . IN) *20.0*DIET/TSUMB

IF (TSUBB.LT.20.0) CS=3. "W(II, IN) *FDIET

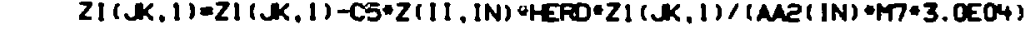

CS-COMPUTES PU ENTERINO ELOODSTIEAM/DAY FROM SOIL

CS-C5*.001 $211(\mathrm{~K}, 1)+C 5$

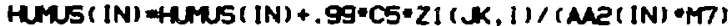

$3+0$ CONT INE

C O(II, IN)=ACCUMLATION OF PU GCING TO PULMONARY REGION

$Q(11, I N)=Q(11, I N)+0.15 * A I T T+Y(11, I N)$

$Q(1 !, I N)=Q(11$, IN $)-Q(11, I N)=4.3+E-04$

IF (O(II,IN).LE.0.0) Q(II, IN) $=0.0$

$L 4=0(11$, IN $) 11$. 3EE $-04 \div 0.001$

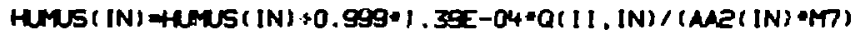

C L5\#RACTION OF PULMONARY ELOOD

LS= (3.6IE-03)・0 (II, IN)

C CTFRACTION OF AIR GI B.OOO

CZ=Y III, IN) $=A I T T \cdot 0.35 \cdot 0.001$

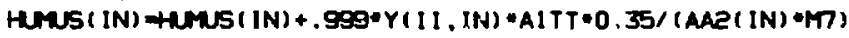

C

CSPU FROM GI ELOOD FROM FOOD

$\mathrm{Cg}=\mathrm{C3} 3.001$

HUMS (IN) =iAMUS ( IN) +.999"C3/(AME( IN) *M7)

HMS ( IN) =HUMS ( IN) $\because Z(I 1$, IN) *HERD

C V(II,IN)=FU IN EODY FROM ALL SOURCES

$V(11, I N)=V(11, I N)+L 4+L 5+C 7+C 9+C 6$

IF (VIII,IN).LE.0.0) $V(I I, I N)=0.0$

C W(11,IN)=PU PER LITER IN MILK

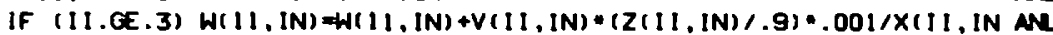
1 )

IF (II.NE.B) CO TO 380

IF (AIII.NE.355) GO TO 380

DO $350 \mathrm{~K}=1.7$

$J=K-1$

THE NEXT TWO STATEMENTS MOVE LIVESTOCK WT. AND ACCT. INTO NEXT AGE C $W(B-J, I N)=W(B+K, I N)$

$V(B-J, I N)=V(B-K, I N)$

350 CONT INUE

WI I , IN) $=23$. $=R R($ IN)

$W(9, I N)=0.0$

$W(10 . I N)=0.0$

$W(12, I N)=0.0$

DO $360 \mathrm{~K}=3.8$

C H( 9.IN)=TOTAL CONTS/CON FOR 3-3 YEAR QLOS

$H(9, I N)=Z(K, I N) * V(K, I N)+H(g, I N)$

$W(10, I N)=W(10, I N)+Z(K, I N) * V(K, I N)$

C WIIE, IN)=TOTAL WEIGT/CON AVE. 3-B YEAR QDS

$W(12, I N)=W(I 2, I N)+Z(K, I N) * W(K$, IN $)$

360 CONT INUE

C W( $9, I N]=C O W N S / C O N / B R E E D I N G$ HEFD

$W(9, I N)=W(9, I N) /(I-Z(1, I N)-Z(Z, I N))$

C $W(10, I N)=A V E$. COUNTS/CON OF TOTAL HERO

$W(10, I N)=W(10, I N)+Z(I, I N) * V(I, I N)+Z(2, I N) * V(2, I N)$

C H(IE,IN)=AVE, WEIGHT/CON OF TOTAL HERO

$W(12, I N)=W(12, I N)+Z(1, I N) * W(1, I N)+Z(E, I N) \bullet W(2, I N)$

C W(13.IN)=FU/KG IN MSCLE TISSE OF HERD

$W(13, I N)=0 . ! 1005 * W(10$, IN $) / W(I E$, IN $)$

C H(14,IN)=FU/KG IN THE LIVER

$W(14, \mathrm{iN})=0.45 * W(10$. IN $) /(0.052 * W(12, I N) * 0.7)$

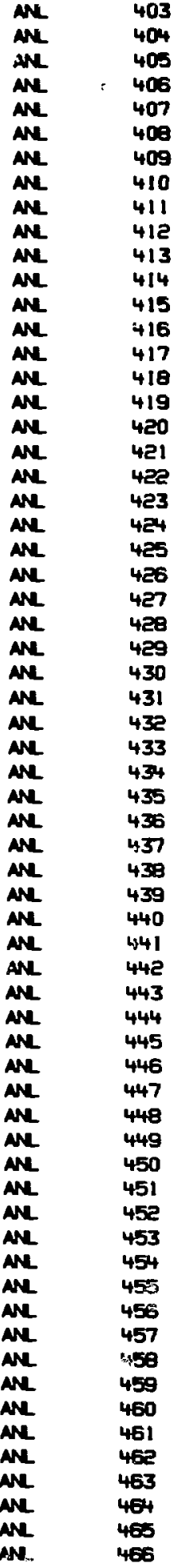


C h(15. IN) Ho of mscle aVailagle to population W? 15. IN) $=0.46 * H(I 2$. IN) *NS (IN) $\bullet P 5($ IN)

C W? 16. IN) PU/KG IN BONE

$W(16 . \mid N)=0.45 * H(10 . I N) /(0.075 * W(12, I N))$

C V(1.IN ISPU IN NEW BOFN CALVES

$V(1, I N)=W(9$, IN $) \cdot 0.001$

PRINT 490

PRINT 5E0, (W(I,IN), I=1,8)

PRINT 500

PAINT 5Z0, (V (I, IN), $1=1, e$ )

HFVSP ( IN) \#RVST ( IN)

SPAP (IN) =SPAS (IN)

SAP (IN) =SPAM IN)

HRVST $($ IN) $=1.0$

SPAS (IN) $=0.0$

SPAN (IN) $=0.0$

TOTSF $(I N)=1$.

IF (AMOD (AI I , XERE) . NE.0.0)GOTO370

FRINT 410, X-ERE

FRINT 420

PRINT 430

PRINT 440, (RR( 1$), 1=1.41$

PRINT 450, (N5 (i), $1=1,4)$

PRINT 460, (AME( $(1), I=1,4)$

PRINT 470, (FSUFF $(1), 1=1,4)$

PRINT 480, (PS (I) $, 1=1,4)$

PRINT 500

PRINT 520, (V (I,IN) $I=1,8)$

PRINT SIO

PRINT 5:0, (Q(1, IN) $, 1 * 1,8)$

PRINT 530

PRINT 540, (W(I, IN), I=9, 16), V(I, IN

370 CONTINE

380 CONTINE

$H(I 1, I N)=0.0$

300 CONTINEE

RETURA

C

400 FOFMAT (EF 10.0)

410 FOFAT (1H-. -RTNANT QUTPUT YEAR OF OUTPUT $=\bullet$, E9.3)

420 FOFUT (1HD, "ANIMAL TYFE SIMLATED")

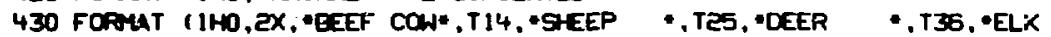

$1 \bullet$

440 FORMAT (2X,E9.3,T14,E9.3,T25.E9.3.T36.E9.3,T50, MATURE hEIGHT RATI AML 10 TO MATURE WEIGHT OF EEEF CON*)

450 FOFMT (2X,E9.3,T14,E9.3,T25,E9.3,T36,E9.3, T50, -NUEBER OF ANIMN U AN INI TS /CATECOPY")

460 FOFAT (EX,E9.3,T14,E9.3,TZ5.E9.3,T36,E9.3, T50, FFACTION OF REGION AN

I GRAZED*)

470 FORMAT (2X,E9, 3, T14,E9.3,T25,E9.3,T36,E9, 3, T50, .FRACTION OF SUPPLE AN IMENTARY FEED*)

480 FOFMT (2X,E9.3,T14,E9.3,T25,E9.3,T35,E9.3, T50, *FRACTION OF MLCLE AM 1 TISSUE ANC ORGANS CONSUMED*)

4O0 FOPMAT (1HD 3X. ANIMAL BIOMASSES KG FOR ACES CLASCES

$1(, 8) * 1$

500 FOPMAT (1HO,3X."ACTIVITY ENTERING ORGANISM FROM ALL SOURCES (VII,I ANE (IN), $I=1,8) \cdot 1$

ANe

510 FOFMT (1HD,3X, ACCUMLATED ACTIVITY ENTERING FROM PULMONARY REGIO ANL IN(QC I, IN), I $=1.8) * 1$

ANe

520 FOFMT (3X,E10.3, TI4,E10.3, TES, E10.3,T38,E10.3, T50,E10.3, TE2, E10.3 AN2

1 .T74,E10,3,TES,E10.3,TSB,AB)

ANL

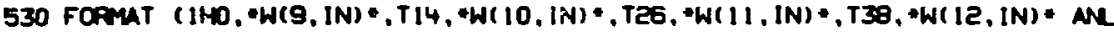

$1 . T 50, * W(13, I N) *, T E 2, * W(14, I N) \bullet, T 74, * W(15,1 N) \cdot, T E 5, * W(16, I N) *$, TSB, AN

$2=V(1, I N)+1$

Me

4E?

468

469

470

4.1

472

473

474

475

476

477

478

479

480

481

482

483

484

485

486

487

483

489

490

491

492

493

494

495

496

497

493

499

500

501

502

503

504

505

506

507

509

509

E:0

511

512

513

514

515

516

517

518

519

520

521

522

523

524

525

525

527

520

529

530 


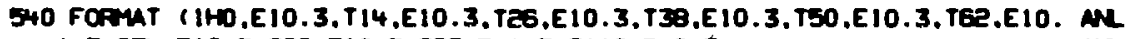
1 3.T74,E10.3.TE6.E10.3.T98.E10.3.T110.E10.31 


\section{APPENDIX F}

PLANT NUMBER POSITIONS FOR BIOLOGICAL TRANSPORT MODEL

\section{WARM SEASON PLANTS}

1. Corn and Sorghum

2. Annual Vegetables and Grains

3. Pasture Grasses

4. Fruit Trees

5. Pinon-Juniper

6. Deciduous Shrubs

7. Annual Grasses and Herbs

8. Warm Season Grasses

9. Evergreen Shrubs

10. Deciduous Trees

\section{COOL SEASON PLANTS}

11. Alfalfa

12. Annual Vegetables and Grains

13. Pasture Grasses

14. Douglas Fir

15. Ponderosa Pine

16. Deciduous Shrubs

17. Spruce-Fir

18. Cool Season Grasses

19. Annual Grasses and Herbs

20. Evergreen Shrubs

21. Deciduous Trees 


\section{APPENDIX G}

\section{CODE DOCUMENTATION OF TRU BIOLOGICAL TRANSPORT MODEL}

\section{INTRODUCTION}

Code documentation of the TRU Biological Transport Model consists of three parts:

- addition of supplementary commentary into the computer code itself to clarify operation

- reworking of input-output options to accommodate specific needs of the user

- a description of process and state equations and boundary conditions

The commentary will be limited to identifying specific locations within the code which can be used in conjunction with this document to describe the operation of the model. Input-output options will be discussed by similar arrangement. In order to facilitate the description of such a lengthy code it will be necessary to follow the order of the code instruction as much as possible, starting with the main program and proceeding with the subroutines in the order presented in the code; it is also assumed that the reader will reference the operating instructions and variable library of the model for greater detail in some instances.

\section{A. Program Plants (Inp, Out)}

\section{SECTION 1: Array Specification and Initial Input Requiremento (statements PLA: 1-36)}

All arrays have been previously defined; ${ }^{1}$ most of them apply to the 21 plant type options selected for simulation. They are either defined as input or are initialized to a given value. The DATA statement (17) defines the number of days present in a given month. All input designated in this section is required for proper code operation. A zero input value suffices in all cases to exclude a certain plant from consideration or delete certain options. Other input pertains to climatic characteristics for the region of interest and for a reference site required to establish certain characteristics when records are not available for a given site. More information pertaining to climate and climatic input variables will be discussed in other portions of this document. The last input requirement in this section pertains to the simulation of TRU activity in the atmosphere for a chronic and/or an acute event. CHRAC is an intercept value for the first day of a chronic event while the values CHRSY, CHREY, CHRSD, CHRED are values referring to the extent (in years) for such an event, and to the number of days within the year when such an event shall take place, respectively. Either or both conditions can be made continuous for the entire simulation period or portions thereof by input specification. This information is used in establishing boundary conditions in duration (years and days within a year) and Activity (activity $/ \mathrm{m}^{\circ}$ in air) in conjunction with Function Airac to be described in Section 3 of this document.

Inputs (CYR and DURC) are utilized to simulate acute air contaminating events (maximum of 64 days for any given year or combination of years (maximum of 16 yearly events)). TRU air concentrations for a given day on a given year are also determined by card input as described in the next section. 


\section{SECTION 2: Optional Input, Initialization, and Characterization of Root Zone (statements PLA: 37-148)}

(Statements PLA: $37-45$ )

If an acute event involving TRU release to the atmosphere is to be simulated, then Input (CYR $=$ IYR) specifies the selected year(s) for such events and the initial day of TRU release for each year are specified by arrays YRCNT and DAIC, respectively, for year and initial day of prescribed events. The array $A M A C$ is required for storing via card input the TRU activity for each day of a given year as specified by Input (CYR = IYR; DURC = IDUR) and limited by the size of the array. ${ }^{2}$ Activity can be specified in activity units or as count rate information.

(Statements PLA: 46-48)

The inputs (F1, W1, H1) relating to water holding characteristics of various soils are converted from percentage of held water to $\mathrm{mm}$ water, present in the upper $250 \mathrm{~mm}$ of soil by an appropriate factor $(2.5=250 \times \mathrm{P} / 100$, where $\mathrm{P}=$ percentage of water in soil, w/w $)$.

(Statements PLA: 51-66)

The number of plants included in the simulation run and specific plants considered are identified for output through use of array $R G C$. Plants are also ranked according to order of appearance in this array.

(Statements PLA: 67-80)

Inputs (FFP, SDPTH, UPTKE, XPLNT, XHERB) are used to specify climatic, soil, output, and subroutine access information. SOILF has the equation:

$\mathrm{SOILF}=0.632 /(1.0-\operatorname{EXP}(-0.004 * \mathrm{SDPTH}))$

where SDPTH = soil depth in $\mathrm{mm}$, describes a function which at $250<$ SDPTH calculates the fraction of actively absorbing root present in the upper $250 \mathrm{~mm}$ of soil. Values of SDPTH as small as $2 \mathrm{~mm}$ soil depth are used for calculating water utilization (Section 3). Soil depths shallower than $250 \mathrm{~mm}$ are assumed to contain all absorbing roots at that depth. Both SOILF and SOILP, its reciprocal, are used to determine other root characteristics to be described.

(Statements PLA: 81-86)

If different plants are to have different rooting depth, or if root depth information is available, such information may be entered via card input by specifying the depth for a given plant in the array $R C O D E .^{:}$The number of soil levels in $250 \mathrm{~mm}$ increments (IICDE) is determined from $R C O D E$, and this value is used to input soil characteristics for each level of depth. The soil activity, $Z 1$, the root fraction $Z F$, and the uptake coefficient $Z U$ for each $250 \mathrm{~mm}$ soil increment 
beneath a given plant is then entered by card input. The units for these parameters have been presented. ${ }^{1}$

(Statements PLA: 87-104)

If all plants are to have the same rooting depth as specified by SDPTH $>1.0$, then the number of soil increments are determined (IICDE) using this input value. RCODE is calculated from SDPTH so that the rooting depth for all plants in the simulation is the same. Furthermore, all values of $\mathrm{ZU}=\mathrm{UPTKE}$; all values of $\mathrm{Z1}=\mathrm{Y} 1$ as specified by card input. ${ }^{1}$ The fraction of roots in each $250 \mathrm{~mm}$ soil level is determined by using equation (1) for each level present, and calculating the difference between successive levels. If there are only 2 soil levels specified (the uppermost being assigned to the $0-2$ depositional horizon), then all of the root fraction is assigned to the second soil level as described previously. The uptake coefficient for the last level is adjusted if it is not totally occupied by roots; this is necessary because the code operates with whole values of soil levels. This fraction is determined by finding the fractional difference between RNDD and REEL in the code. The first level is also reserved for the $(0-2) \mathrm{cm}$ depth when soil level information is obtained from card input; both the root fraction $\mathrm{ZF}$, and the uptake coefficient $\mathrm{ZU}$ are assumed equal to zero in this case. The TRU activity in the upper level $\mathrm{Z} 1$ is assigned a value depending on user needs; it is used for resuspension-deposition simulation of TRU, and as a driving function for movement of TRU into lower soil horizons to be described.

(Statements PLA: 105-146)

Arrays and other variables not used as input are initialized to zero or appropriate values.

(Statements PLA: 147-149)

Bud break and leaf drop for deciduous trees and shrubs is calculated by subtracting or adding one-half of the frost free period (FFP) for a given region to day number 210 , respecitively. The latter represents the approximate warmest calendrical day in most of the United States on a longterm average basis.

\section{SECTION 3: Calendrical, TRU Air Concentration, Precipitation, Irrigation, Hydrological, Temperature Characteristics, and Initial Output (Statements PLA:)}

(Statements PLA: 153-159)

If irrigated plants are to be simulated as determined by (RIRRC), then harvesting (CUTA), applied water (AWATA), and the time of water application (DIRA) are entered via card input. The first column of input for (CUTA) and (DIRA) are reserved for harvest, and planting date, respectively.

(Statements PLA: 160-163)

The random number seed (RANDN) is assigned an integer value (I7) for use in a DO loop. The mid range solar radiation value $(A)$ is calculated as the difference of yearly maximum and minimum solar radiation (T1 - T1) divided by 2 . The mid range monthly temperature, ${ }^{\circ} \mathrm{F},(\mathrm{B})$ is calculated as the difference of average maximum and minimum monthly temperature, ${ }^{\circ} \mathrm{F},(\mathrm{T} 4-$ T3) divided by 2 . 
Initialization or seeding of the random number generator $R A N D O M$ is provided by iteration using a random number input (RANDN $=17$ ) provided by the user. This scheme provides a different set of numbers used for climatological determinations described later in this section.

(Statements PLA: 166-167)

The years of simulation (Y2) is given an integer value (IJ) for use in a DO loop. A year counter (ICYR) is initialized to 1 .

(Statement PLA: 168)

This statement marks the establishment of the requested simulation period in years as obtained through input $(\mathrm{Y} 2=\mathrm{IJ})$.

(Statements PLA: 169-173)

If an acute event is requested for any year or combination of years as specified in (YRCNT), then after such an event has been completed the index value (ICYR) is incremented by one to specify the next year when another event is to be recorded...etc.

(Statement PLA: 176)

This statement sets the initial year of simulation to zero. In most cases the program is allowed to proceed through the first year of the simulation before requesting any options to remove any extraneous influence caused by setting of initial conditions. Agricultural crop simulation is an exception to this rule.

(Statements PLA: 175-239)

Initial output is provided for the first year only. Included in this output is information concerning user options. If any or all subroutines are used, or if plants are entered in competition or succession, or if irrigation will be used, or if a chronic or contaminating event is simulated, the output will reflect user needs. Temperature, precipitation, and soil characteristics are printed under those headings. Chronic event and irrigation information will appear only if simulated in the model.

(Statements PLA: 240-248)

$(A Q)$ is set equal to (I1) for use in daily output. Annual net production (WURAT) for specific plant, annual average temperature (TAV), annual cumulative precipitation (P1), and the real value of day number (K3) are set to zero. Day counter for irrigation (IRG) and day counter for harvesting schedule (ICA) are set to 2.

(Statement PLA: 249)

This statement marks the beginning of a calendrical year ( 365 days) for any given year of code operation. 
The simulation of TRU air concentrations in a given region is facilitated by (AIRCA) for both a chronic and an acute event. If an acute event is requested, the TRU activity is entered from storage (AIRCA $=$ AMAC) if the year (AI1 and the day number AIII) meet the specifications set forth in (YRCNT), (DAIC), and (DAIC + DURC). These operations may be circumvented by making the input value $(\mathrm{CYR}=0.0)$.

If a chronic event is to be simulated singly or in combination with an acute event...i.e., then the function (AIRAC(CHRAC,AIl, AIII)) is executed provided the year (AII) and the day number (AII) are within the limits set forth by inputs (CHRSY, CHREY), and inputs (CHRSD, CHRED), respectively. This operation may be circumvented by zeroing these inputs. The summation of all events into AIRCA makes it possible to add these TRU activity contributions to the air with that contributed from resuspension of TRU from the surface of soils and plants to be discussed in another section of this report. The function AIRAC is provided by the user and subsituted in the code at the appropriate location (1340-1343). A dummy function is provided in the code when tiis function is not being called.

(Statements PLA: 261-275)

The day number (III) within a given year (I1) is assigned to one of he calendrical months, depending on its value. The day number for the end of each month is set at $21,59,90,120,151$, $181,212,243,273,304,334$, and 365 days, respectively. February is assigned 27 days for all simulations and is not corrected for leap years. If the day number (III) equals the first of any month, it is assigned a month number (I) which is used in allocating monthly climate.

(Statement PLA: 277)

Unadjusted monthly precipitation is obtained by taking the product of monthly fraction (X) with the mean annual precipitation $(R)$, and the conversion to $\mathrm{mm}$.

(Statements PLA: 278-280)

Random number counter (Q8) and product of random number and number of days in given month (Q) are initialized to zero.

(Statements PLA: 281-289)

The number of precipitation events (NRE) in a given month (NDAY) are allocated to days within the month by selection of a random number from an even distribution (RANDOM), and taking the product of this number $(0 \leq \mathrm{RANDOM} \leq 1)$ with (NDAY); this value is allocated to (Q). In addition, (NRE) random numbers are selected, and summed (Q8) for use in precipitation allocation to the specific events determined in $Q(L),(1 \leq \mathrm{L} \leq \mathrm{NRE})$; precipitation allocation will be discussed later in this section. Finally, fractional days are converted to the nearest whole day as determined by $(\mathrm{ABC})$ and allocated to $(\mathrm{NN})$.

(Statements PLA: 290-295)

If any of the precipitation days (NN) are equal to each other, then another set of days is considered by reverting to statement PLA: 290 until (NRE) different precipitation days are selected. 
Two random numbers (G4) and (G5) are selected from a normal distribution $\left(\mu=0, \sigma^{2}=1\right)$ for providing variations in monthly temperature and daily precipitation events to be described later in this section. Day counter $(\mathrm{M})$ is incremented by 1 .

\section{(Statements PLA: 305-309)}

Insolation in Langleys/day (AKK) is estimated by using the sum of a sinusoidal function with the minimum insolation (T1) for a given region. The function has limits between zero and the total difference $(A \times 2)$ between minimum and maximum insolation values observed. The function also utilizes the lag factor (D) described previously. The photosynthetic efficiency (M1) is coupled to this function by a proportionality constant until saturation $(0.45 \mathrm{~g}$ photosynthlate/g green biomass) is reached and remains at this value for insolation above 450 Langleys/day.

\section{(Statements PLA: 311-314)}

Mean daily temperatures $\left({ }^{\circ} \mathrm{F}\right)$ for a given region are estimated by using the sum of a sinusoidal function with the minimum daily temperature (T3). The function has limits between zero and total difference $(2 \times \mathrm{B})$ between minimum and maximum temperatures observed. The function also uses the temperature lag factor $(\mathrm{E})$ described previously. Regional mean temperatures are estimated by summing the temperature estimated for a reference site at a given elevation (RELV) with the product of the lapse rate (ALPSR) and the difference in elevation between reference site and the site of interest (RELV - G1) where (G1) is the elevation above sea level of the latter. The temperature is also corrected for the latitude difference (L3) between the reference and site of interest. This correction is set at $1^{\circ} \mathrm{F} /{ }^{\circ}$ latitude difference. All mean temperatures are converted to ${ }^{\circ} \mathrm{C}$ before making a final stochastic adjustment.

(Statements PLA: 315-319)

Variation of estimated monthly mean temperature is provided by generation of the normal random variable $\left(\mathrm{G} 4, \mu=0, \sigma^{2}=1\right)$ which is multiplied by an estimated standara deviation of the temperature statistic (TMXV). Any temperatures falling below $0^{\circ} \mathrm{C}$ are set equal to the latter. Finally, the mean annual temperature (TAV) is calculated using the estimated mean daily temperatures generated.

\section{(Statements PLA: 320-334)}

Precipitation (P) is allocated (NRE) times to a given month corresponding to a given day number $M$ whenever it equals a selected day $(N N)$ described previously. The ratio $(Q / Q 8)$ represents the fraction of the estimated monthly rainfall (DRMM) which is allocated to one of the (NRE) days selected for the event to occur. In addition, the precipitation is modified by adding to the initial allocation the quantity $\left(\mathrm{P}^{*} \mathrm{G5}\right.$ * $\mathrm{PRCV}$ ) where (G5) is generated from a normal distribution as described for (G4), and (PRCV) is the estimated coefficient of variation for a precipitation event when it occurs in the region of interest. The total annual precipitation for a given year (P1) is estimated by summing all daily events. If temperature (T) is equal to zero, then $P$ is expressed as snow accumulation (F7) which is converted to snowmelt (SNM) as a function of temperature (T). If the calculated (SNM) exceeds (F7), then (SNM) is set equal to (F7). 
(Statement PLA: 335)

The potential evapotranspiration rate (E2) is a function of temperature (T).

(Statements PLA: 336-339)

Plant types (LPN) not simulated are excluded from the daily loop (RGC $=0$ ). For the plants considered in the simulation the amount of soil water (WA) present is adjusted for additions due to precipitation (P) and/or snow melt (SNM). Also, air concentration of TRU (AAA) is adjusted for chronic and/or acute contributions (AIRCA).

\section{SECTION 4: Irrigation Option, Soll Water Status, and Evapotranspiration Adjutment (Statements PLA: 341-356)}

(Statements PLA: 341-347)

If irrigation is to be required for any plant (LPN) considered (RIRRC) then input amount (AWATA) is added to the existing soil water (WA) on a given day number (DIRA); the amount is added to the cumulative amount of ppecipitation (P1) for that year. The day counter (AIII) is then set to test the next day number for such an event (IRG).

(Statements PLA: 349-357)

The amount of soil water (WA) is set equal to the holding capacity of the soil (H) if additions exceed that value. The loss of soil water through evapotranspiration (EA) is set equal to the potential evaporation (E2) adjusted for the ratio of available soil water (WA) to the field capacity (F1) of the soil. If soil water is less than the wilt point (W1), (EA) is set to zero; if (WA) is greater then the field capacity, (EA) is set equal to (E2). Soil water losses are adjusted for soil depth with the factor (SOILF) described previously. The adjusted soil water (WA - EA * SOILF) for a given day (AIII) is set equal to the wilt point (W1) if losses render it less than that value. Also, the loss (EA) is adjusted to a value which would not allow soil water (WA) to drop below (W1). Finally, the variable (S1) is set equal to (EA), and (E4) to (EA) for use in the next section.

\section{SECTION 5: Effect of Soll Moioture and Temperature on Growth, and Plant Growth Characteristics (Statements PLA: 358-479)}

(Statements PLA: 358-366)

The determination for deciduousness in simulated plants is initialized (ISTP $=1$ ) which specifies either evergreen trees and shrubs, or herbs, crops, and grasses which shed above ground parts during the off-season. The variable (SCTP) for a given plant (LPN) is initiaiized to simulate an herbaceous plant; the initial value $(\mathrm{SAFP}=5$ ) corresponds to a factor used for determining the effect of soil moisture on the photosynthetic rate of warm season plants. The effect of temperature on photosynthesis (E5) is also set for warm season plants at daily temperature (T) for day (AIII). The effect (E5) is determined through the use of several relationships depending on whether the temperature (T) is above or below the optimum $\left(25^{\circ} \mathrm{C}\right)$ for that plant class. If the plant is of a cool season type (LPN > 10) then values of (SAFP), and (E5) are initialized for this plant set. 
This subsection includes nıodification of any plant type (LPN) if it differs from the initial conditions set forth in the previous subsection; both warm and cool season plant types can be selected by proper matching of (LPN) with the access statement number in the computed (GO TO) Statement.

(Statements PLA: 476-478)

The constant (Q1) in the exponential term for determining the effect of soil moisture on photosynthesis (E7) is calculated using previously obtained values of (SAFP), (F1), and (W1). The value of E7 is limited to a value between zero and one as is (E5).

\section{SECTION 6: Growth Form, Respiration, Photosynthesis, Biomass Allocation, Leaf Biomas, and Plant Life-Span Specification (Statements PLA: 479-718)}

(Statements PLA: 479-499)

The biomass density of photosynthetically active biomass (WN1) is estimated initially as a function of (B11). A respiration biomass increwent (ARP) given in increments of (WN1) is initialized at zero. This increment is added to (WN1) as part of the plant maintenance respiratory loading. The water utilization efficiency (WUTP) is initialized at 350. Also, the fractions of photosynthate translocated to above ground biomass (STRANS) and to below ground biomass (RTRANS) are set to 0.75 and 0.25 , respectively. Photosynthesis (BAP) and respiratory (BCP) gates are initialized inthe open condition(1). The constants (RFP, RXPP, and RAP) in the plant respiration equation are initialized at $0.01,0.161$, and 0.13 , respectively. Finally, the mean lifespan (HF) and the photosynthetic efficiency (PSE) are at 30 years, and 1, respectively; the latter value determines whether a given plant is a Calvin type plant $(\mathrm{PSE}=1)$ or a more efficient Hatch-Slack plant (PSE $=0.5$ ) which is set at twice the efficiency of the former; also, the biomass density (BP) of green and dead standing biomass is calculated for fut ure use in the code. If a plant is of a cool season type ( $L P N>10$ ), then a new set of respiration coefficients ( $R X P$, RXPP, RAP) are initialized for this category.

The (GO TO) statement directs the user to different growth strategies, depending on the plant simulated (LPN).

(Statements PLA: 500-707)

If the values of constants described previously in this section do not apply to a specific plant (LPN) simulated, then required constants are made within the statement subgroups designated. Statements PLA: 501-502, 509-510, 606-607 set limits on the occurrence of photosynthesis (BAP), and respiration (BCP) for harvested crops to correspond to planting (DIRA) and harvesting (CUTA) schedules. The first statement does not allow photosynthesis before planting day number or after the crop has been harvested. The second statement of each subgroup does not allow respiration to occur beyond the harvesting date, or for the initial 15 days after planting. Statements PLA: 520-524, 534-538, 549-553, 573-577, 584-588, 617-621, 631-635, 648-652, 659-663, 684-688, 695-699 quantify photosynthetic (WN1) and respiratory (ARP) biomass density for evergreen and deciduous tree and shrub types included within the code. The calculated photosynthetic biomass density maximum is limited to either all or one-half the calculated yearly running average productivity (TPGNW) for evergreen and deciduous woody plant types, respectively; calculated values of (WND) less than the latter are allowed in either case for 
(WN1). The respiratory biomass density (ARP) is estimated as some multiple of photosynthetic leaf biomass deusity (WN1) for both deciduous and evergreen plant types, however, (ARP) is reduced by (WN1) during the dormancy period with limits set by (SFFP) and (EFFP) for deciduous types. Finally, (ARP) for both plant types is limited to values equal to or less than the sum of above ground (B1) and below ground (R1) biomass densities which is of significarice only during early plant growth.

\section{(Statements PIAA: 708-713)}

The root respiration rate $(\mathrm{AC})$ is set at one-third the above ground green biomass rate for herbaceous type plants and involves the constants (RFP, RXPP, RAP) and temperature (T) already described. The leaf area index (TLAI) is derived from (BP) which represents either the photosynthetic green biomass or in addition may include the dead standing component (D1) described previously. The respiratory biomass density for a given plant (RSBIO = ARP) is set at (WN1) if (ARP) is not utilized in the plant growth strategy. Also, the succession factor (SFAC) is set to one if either the succession module in the code is not accessed (RSCDE $=0$ ) or if the plant type is agricultural and is not competing with other plants simulated (RSS > 12); other nonagricultural plants can also be excluded from succession by the latter control whøn $($ RSCDE $=1)$ for other plants within the simulation. Finally, if the plant type is deciduous (ISTP $=0$ ), then the photosynthetic biomass density (WN1) is set to one outside the growing season.

\section{SECTION 7: Photosynthesis, Respiration, Water Utilization Efficiency, Net Produstivity, Photosynthate Allocation (Statements PLA: 719.745)}

(Statements PLA: 719-735)

(iross daily areal photosynthesis (B5) is estimated from the product (WN1) * (BAP) * (Mi) * (E5) * (E7) * CFAC * SFAC which has been described. Net daily areal photosynthesis is estimated by subtracting the respiratory or maintenance term from (B5): (WN1 + ARP) * (RFP) * $(\mathrm{EXP}(\mathrm{RXPP}) * \mathrm{~T}) *(\mathrm{RAP}) *(\mathrm{BCP}) * \mathrm{CFAC} * \mathrm{SFAC}$. The daily evapotranspiration rate $(\mathrm{T} 5=$ $\mathrm{T} 5 \mathrm{P}^{*} \mathrm{CFAC} * \mathrm{SFAC}$ ) is estimated for comparison with the amount required (T6) for the potential net production estimated. The latter is estimated from an interaction term (E5 ${ }^{*} \mathbf{E} 7 \geq 0.01$ ) between temperature and soil water effects on photosynthesis, an estimated maximum water utilization efficiency (WUTP) for a specific plant, the relative photosynthetic efficiency; and the potential net photosynthesis (B5):

$\mathrm{T} 6=(\mathrm{C}) *(\mathrm{PSE})$,

where

$\mathrm{C}=($ WUTP $) /(\mathrm{E} 5 * \mathrm{E} 7)$.

If the water evapotranspiration requirement (T6) exceeds the estimated evapotranspiration (T5), then (EWAT) is reduced to the maximum allowable under these conditions:

$\mathrm{EWAT}=(\mathrm{T} 5 / \mathrm{T} 6) *(\mathrm{~B} 5)$.

(EWAT) is further adjusted for competition with other plants in succession (SFAC) or for competition within the niche (CFAC) to be described in a later section of the report. I: the potential 
net photosynthesis is negative, (EWAT) is made equal to it (B5). Net production for a given year (WURAT) is obtained by summation of daily productivities (EWAT).

(Statements PLA: 736-745)

Photosynthate is transferred to above (B1) and below (R1) ground biomass compartments via increments (B5) and (B7), respectively, using (STRANS) and (RTRANS). However, if (EWAT) is negative, respiratory losses are made on a proportionality basis using (B1) and (R1) as factors. Eventual losses or gains of biomass are reflected in (B1) and (R1) for a given day (AIII) of the simulation.

\section{SECTION 8: Biomess Losses due to Respiration, Harvesting, and Mortality; Humus For- mation, and Control Options (Statements PLA: 747-980)}

(Statements PLA: 747-748)

The effective life span for successional purposes (RSCF) in initialized to equal the average life span (HF) of a given plant type (LPN). The mortality loss factor (AP) for a given plant is based on the average life span (HF).

(Statements PLA: 749-750)

The computed (GO TO) statement controls access to specific plant biomass loss strategies.

(Statements PLA: 751-950)

For harvested crops and grasses of cool and warm season types (PLA: 754, 770, 774-788, 793$805,855-867,871-885,888-900$ mortality loss of above ground biomass (D3) is estimated at a value proportional to (B1) within a certain temperature regime specific to the plant type (LPN). If the value is outside, the temperature range (D3) is estimated from the product (D3) * (R1/B1). (RD3) for pasture grasses and alfalfa are estimated from the mortality loss factor (AP) and (R1). Also, a respiration term $(A C) *(R 1)$ is employed for these plant types to account for the uncoupling of maintenance biomass ( $A R P=0$ ) for below ground portions in the photosynthesis equations described earlier. Crop harvesting losses at prescribed dates (CUTA) are estimated to augment (D3) by (B1/10) and (RD3) by (R1). Also, TRU concentrations (Z3) and (Z2) are set to zero while (Z4) and (Z4R) are incremented by (Z3/10) and (Z2), respectively. Grass harvesting losses reduce (B1) to $20 \mathrm{~g} / \mathrm{m}^{2}$ if the preharvest value of (B1) exceeds the latter initially. Proportional amounts of (Z4) and (D1) are also removed from preharvest values when applicable. Also, the values (B1 = $1),(R 1=1),(D 1=1)$, and (D3 = 0) are specified for crops outside of the growing season as specified by (DIRA) and (CUTA) for a given plant. Mortality loss schemes for perennial grasses (Statements PLA: 835-839, 926-930) are identical to those of pasture grasses. Annual grasses and herbs (Statements PLA: 827-832, 934-938) have mortality schemes similar to harvested crops, but differ in that (RD3) is augmented by the product (AP) * (B1) for these plant types. The mortality increment (D3) for evergreen trees and shrubs (Statements PLA: 815-817, 843-845, 904-905, 909-911, 921-922, 942-943) is composed of an exponential needle or leaf less involving (WN1) and a mortality loss (AP) * (B1). The former loss assumes continuous leaf loss throughout a given year. Root mortality (RD3) is estimated from the products (D3) * (R1/B1) as described previously for other plant types. Mortality increment (D3) for deciduous trees and shrubs (Statements PLA: $809-811,821-823,848-850,915-917,946-949)$ consists of the product (B1) * (AP) except at the end of the frost-free period (EFFP) when (D3) is augmented by the total leaf biomass (WN1). Root mortality increment (RD3) is calculated as for evergreen plants of this type. 
(D3) and (RD3) are set to zero if they exceed (B1) and (R1), resfectively. The estimates (B1), (R1), (D1), and (RD1) are then adjusted on a daily basis by the mortality increments (D3) and (RD3).

(Statements PLA: 959-966)

Humus formation is estimated from a temperature $(\mathrm{H} 2)$ and soil moisture $(\mathrm{H} 3)$ effect on the decomposition rate of (D1) and (RD1). Both above ground produced humus (H1) and root humus (RH1) are augmented daily by the products (H2) * (H3) * (D1) and (H2) * (H3) * (RD1), respectively; conversely, (D1) and (RD1) are reduced by these amounts on a daily basis. Humus decomposition is estimated in daily increments $(\mathrm{AC}){ }^{*}(\mathrm{H} 1)$ or $(\mathrm{AC}){ }^{*}(\mathrm{RH} 1)$ depending on the type.

(Statements PLA: 967-972)

All estimates (B1, R1, D1, RD1, H1, RH1) are set to one if values fall below this value.

(Statements PLA: 973-977)

The initial year of the simulation $(\mathrm{I} 1=0)$, plant $(\mathrm{RGC}=3)$, minimum biomass $(\mathrm{B} 1=1)$, and Subroutine Forman access statement for a specific year $($ XPLNT $=11)$ all control calling of Subroutine Forman for all plant types, but primarily for those of an herbaceous nature where daily stand structure is desired.

(Statement PLA: 979)

This statement marks the end of the daily plant module loop indexed by (LPN).

(Statement PLA: 980)

Access statement to Subroutine Rmnant depends on the value of (RHERB).

\section{SECTION 9: Niche Competition, TRU Resuspension, Transport and Activity (Statements PLA: 981-1133)}

(Statements PLA: 981"1000)

If similar plant types such as warm and cool season grasses are interacted (same value for RSS!, then a weighted factor (CXFAC) is determined to control photosynthate production on a daily basis as described earlier. This factor is estimated from a consideration of the fractional daily growth rate $(B 5+B 7) /(W N D)$. The fractional daily growth rate

$(\mathrm{B} 5+\mathrm{B} 7) /(\mathrm{WND} * \mathrm{RSCF})$,

is summed over all plants with equal (RSS < 12) to obtain (CFAC) for day (AIII). (CFAC) is later equated to:

$\mathrm{CFAC}=(\mathrm{CXFAC}) /(\mathrm{CFAC})$,

to obtain the desired factor. If production is negative (B5 $<0)$, then this interaction is circumvented. 
(Statements PLA: 1003-1016)

Weighted average vegetative cover (CVTT) and air concentration (AITT) are obtained by summing the products:

$(\mathrm{CCVV}) *(\mathrm{CVMX})$, and $(\mathrm{A} 1) *(\mathrm{CVMX})$,

respectively, where (CCVV) is the estimated ground cover of a specific plant, (A1) is the immediate TRU air concentration surrounding the plant type, and (CVMX) is the fractional successional status of that plant to be described within the succession module. If succession is not considered $(\mathrm{RSCDE}=0$ ) or for the initial year $(\mathrm{I} 1=0)$; both $(\mathrm{CVTT})$ and $(\mathrm{A} 1 \mathrm{TT})$ are allowed to assume their specific values (CCVV) and (A1), respectively. A resuspension factor for TPU (RSUSF) is estimated from (CVTT) by an exponential equation:

RSUSP $=2.5^{*} \operatorname{EXP}\left(-4.6^{*} \mathrm{CVTT}\right) * 10^{-8}$

CVTT is not allowed to become larger than one.

(Statement PLA: 1013)

A second plant loop is entered (LPN).

(Statements PLA: 1014-1021)

If (CVTT) and (A1'TT) are not estimated by previous means, then they are allowed to assume values for a specific plant (CCVV) and (A1), respectively. (RSUSP) is estimated in the same manner as before.

(Statements PLA: 1022-1039)

The first soil layer $(0-2 \mathrm{~cm}),(Z 1)$, is depleted of TKU by two processes:

$(\operatorname{RSUSP}) *(\mathrm{Z} ?)$

and

$(0.693 / 700) *(\mathrm{Z} 1)$

representing losses via resuspension of particles to the atmosphere, and that due to leaching of TRU to the next scil horizon, respectively. The surface layer is augmented by deposition of particles which are estimated to be proportional to (A1TT) described assuming a constant $450 \mathrm{~m} / \mathrm{day}$ deposition velocity, and a $2 \mathrm{~cm}$ soil mixing layer; the second soil layer (Z1) is augmented by TRU leached from the top layer. The total amount of TRU resuspended from bare ground (A1Z) is estimated from (RSUSP), (Z1), and the estimated weight in grams of soil $2 \mathrm{~cm}$ deep and $1 \mathrm{~m}^{2}$ area. On day numbers $(\mathrm{AII})$ when precipitation is absent $(\mathrm{P}=0)$ the surface layer $(\mathrm{Z1})$ is augmented by losses from plant surfaces (A2) and from the release of humus TRU to this layer. The loss rate of TRU from plant surfaces is based on an estimated 30-day retention half-life for particles under dry conditions. Air concentration (A1) is regenerated daily from contributions of (A2) surface humus (Z5), and that contributed from bare soil (AIZ); addition of TRU to air from other sources 
(AAA) is also considered when applicable, and is discussed in a later section. The amount of surface deposition of TRU on plant surfaces is determined by the following relationships:

$$
\mathrm{A} 2=-(\operatorname{RSUSP}) *(\mathrm{~A} 2)-(0.693 / 30) *(\mathrm{~A} 2)+(0.0021) *(\mathrm{~A} 1 \mathrm{TT}) *(\mathrm{TLAI}) *(0.67)
$$

in which deposition is mide dependent on the leaf area index of plant (LPN) and allows for $67 \%$ initial retention of deposited particles.

On day numbers when precipitation occurs $(P>0)$ resuspension is not allowed to occur, and depesition-retention characteristics are related to the amount of precipitation $(P)$ in addition to other factors described earlier. Under these conditions the top layer of soil is augmented by as much as 0.3 of the deposit on plant surfaces (A2) being modified the value of ( $P$ ) using the following relationship:

$(0.3)^{*}(1-\operatorname{EXP}(-0.6518 P))$.

Similarly, leach losses from the top soil layer into the second is also controlled by the amount of (P):

$(0.01) *(1-\operatorname{EXP}(-0.6518 P))$

Also, a minimum of 0.7 of (A1TT) is deposited on soil and plant surfaces, depending on the value of (P) as before.

(Statements PLA: 1041-1088)

The extent of root penetration into successive soil levels (RPNTP) is estimated with an exponential function relating the deepest penetration allowed in the code $(7752 \mathrm{~mm})$ to the total number of days since the start of growth (AI1P). If annuals are simulated, then (AI1P) is set to equal the respective day number in a given year (AIII) for RSCF $\leq 9.0$.

The extent of root penetration (RPNTP) is used via the number of soil layers (IICDE) to determine whether 'TRU is absorbeci by roots (Z2) from any given layer as denoted by the root gate (SP). The amount of 'TRU absorbed (RGAIN) is determined by the following relationship a specific soil level for a given plant $(\mathrm{LPN})$ when $(\mathrm{SP}=1)$ :

$\mathrm{RGAIN}=(\mathrm{ZU}) *(\mathrm{ZF}) *(\mathrm{~T} 5 \mathrm{P}) *(\mathrm{WND}) *(\mathrm{Z1})$

which is used to increment (Z2) on a daily basis and decrease the specific soil layer (Z1) by the same amount. However, each soil layer $(\mathrm{Z} 1)$ is augmented by the mineralization of root humus TRU(Z5R) at an estimated rate (AC) discussed earlier.

The movement of TRU from below ground biomass (Z2) to above ground portions (Z3) is estimated by (ADD) which is related to below ground translocation of photosynthate on a daily basis (B7) for a given plant; both positive and negative values of (B7) are considered to simulate exchange of TRU between above (B111) and below ground (R111) biomass components. Transport is controlled by small values of $(\mathrm{B} 1 \leq 1)$, and maintenance biomass, (T111 $\leq \mathrm{B} 1)$. Movement of TRU from roots (Z2) to above ground biomass (Z3) is estimated as:

$(\mathrm{ADD}) *(\mathrm{Z2}) *(\mathrm{~B} 111) /(\mathrm{B} 111+\mathrm{R} 111)$ 
and gain from (Z3) as:

$(\mathrm{ADD}) *(\mathrm{Z} 3) *(\mathrm{R} 111) /(\mathrm{B} 111+\mathrm{R} 111)$.

The reverse is true of TRU transport to and from below ground. Above ground biomass (Z3) is corrected for mortality losses:

$(\mathrm{Z3}) *(\mathrm{D} 3 / \mathrm{B} 1)$, as is that for below ground parts:

$(\mathrm{Z2}) *(\mathrm{RD} 3 / \mathrm{B} 1)$;

Both ( $Z 3 \geq 0$ ); $(Z 2 \geq 0$ ) conditions must be satisfied for proper code operation. The TRU activity in dead standing biomass (Z4) is augmented from (Z3) losses and decreased by the product ( $\mathrm{H} 2)$ * $(\mathrm{H} 3)^{*}(\mathrm{Z4})$ for a given plant type (LPN): Z4R is similarly calculated for dead standing root biomass. Changes of TRU in humus (Z5) is governed by losses due to respiration $\left(\mathrm{AC}{ }^{*} \mathrm{Z5}\right)$ and resuspension (RSUSP * Z5); and is augmented by gains from (Z3) in its conversion to humus. Z5R is similarly estimated for below ground humus. (Z9) and (Z9R) are activity accumulators of TRU, having no other function than to sum up all TRU activity which has been recycled to the soil via humus of both above and below origin. (Z6) and (Z7) estimate the specific activity of TRU in above and below ground (B1, R1) biomass, respectively; (X8) and (Z8) estimate TRU accumulation factors for above and below ground components, respectively. In like manner (Z7P) and (Z7PR) estimate the TRU specific activity in dead standing above and below ground biomass components. Finally, the factors (A22) and (A23) estimate TRU specific activity and accumulation factor for surface deposition on plant leaf biomass (Z77) is the TRU accumulation factor for internal and external activity in above ground biomass.

(Statements PLA: 1089-1091)

After calculation of the total number of days elapsed (TP) since the beginning of the simulation, the decision to print factors generated on a daily basis is controlled by the user through the equality $(\mathrm{AQ}=\mathrm{XPLNT})$ for any given year of the simulation (I1).

(Statements PLA: 1091-1117)

Daily output is provided if the year number (AQ) is equal to the year specified for daily output (PLNT). Headings provide variable names to conserve time and space (see variable library for definition of headings). Day number and plant number (LPN) are provided to facilitate easier reading.

(Statement PLA: 1118)

This statement marks the end of the daily loop (AIII).

SECTION 10: Running Average Yearly Production, Prorated Leaf Biomass, Plant Succession, Print Statements and Forman Access (Statements PLA: 1119-1257)

(Statement PLA: 1119)

The cumulative sum of yearly iterations (SYAV) is incremented by 1 . 
The next plant loop (LPN) in the program is used to calculate the fraction of potential leaf biomass (W11) from above ground biomass density (B11) which for woody plants (SCTP $=0$ ) is compared with the current production of that plant (WUYAW); the latter is set equal to (W11) if (W11 < WUYAW). The sum of all yearly production estimates (WYAV) is divided by cumulative years of simulation (SYAV) to obtain the running yearly average biomass production (TPGNW $\geq 0.001$ ) which is used to prorate leaf biomass for the following year of simulation. Plant cover for a given plant is estimated by:

$\mathrm{CCCC}=(1-\operatorname{EXP}(-1.54 * \mathrm{TPGNW})) * \mathrm{C} 6$,

although better estimates are obtained in Subroutine Forman for trees.

(Statements PLA: 1135-1180)

Two or more plants simulated under yearly interaction (RSCDE $=1$ ) are treated in the succession module. Plants are ranked (RANK) according to their specific value of (STBR) which is a yearly running average of:

$(\mathrm{R} 1+\mathrm{B} 1+\mathrm{D} 1+\mathrm{RD} 1+\mathrm{H} 1+\mathrm{RH} 1) /(\mathrm{RSCF})$

plants with (RSS > 12) which are generally agricultural crops are not subjected to this treatment $(\mathrm{CFAC}=1, \mathrm{SFAC}=1, \mathrm{RANK}=1)$. The highest value of $(\mathrm{STBR}=\mathrm{STBMX}$ for RANK $=1)$ is used to determine a succession factor (SFAC) for all plants concerned. If agricultural crops are included as part of the simulation, individual plant acreage (CVMX) is used to calculate the total cover (CVAG) of all crops through the use of (ACRES) for such plants (RSS > 12). Caution should be used in allocating acreage assignments to that they do not exceed study area acreage. (CVMX) for a given crop plant is estimated as:

$(\mathrm{ACRES}) *(4047) *(\mathrm{CCVV}) /\left(\left(3.07 \times 10^{*} * \mathrm{CVTT}\right)\right.$

for use in determining (RSUSP) and (A1TT) described earlier. (CVAG) is used to adjust succession factors (SFAC) for specific noncrop plants in the succession module, and will be described later in this section.

(Statements PLA: 1181-1221)

The succession factor for a given plant (SFAC) is initially allocated by the value of rank. Plant cover (CCVV) for each plant and a succession factor cover term (SFCT) which is cumulative of:

$(1-\mathrm{SFCT}) *(\mathrm{CCVV})$

The latter produces a series of factors according to (RANK) for all plants in the series. (SFAC) is normalized by (CCVV) for a given plant and is affected by the term:

(STBR)/(STBMX).

The sum (SFFB) represents the sum of (SFAC). Another adjustment is made on SFAC: 
$\mathrm{SFAC}=(\mathrm{SFAC}) / \mathrm{SFFB}$

and the final adjustment is performed to account for crops cover:

$\mathrm{SFAC}=(\mathrm{SFAC}) *(1-\mathrm{CVAG})$

the final summation (SFFC) is obtained. The latter is used to calculate the fractional cover contribution of each plant placed in competition:

CVMX = SFAC/SFFC,

which is then used to estimate (A1TT), and (CVTT) as described previously.

(Statements PLA: 1224-1236)

Yearly output is provided for each plant simulated in the model. Headings provide variable names to conserve time and space (see variable library for definitions of variable names). Plant number and year are included to facilitate easier reading.

(Statements PLA: 1238-1249)

Calculations for specific activity values (Z7P, Z77, A22, A23) are estimated for output.

(Staternents PLA: 1253-1254)

Access to Subroutine Forman (RGC $>2$ ) is optioned.

SECTION 11: Input Output Format Specifications (Statements PLA: 1260-1338)

\section{B. Subroutine Forman}

\section{SECTION 1: Array Specifications, and Input Requirements (Statements FMG: 1-17)}

All arrays in Common, and those in Dimension statements have been defined previously; the latter apply primarily to structural characteristics of a given plant ( $\mathrm{JK}$ ) for a minimum of 60 different diameter classes. They are derived from biomass and morphological variations among the 22 plant types (JK).

If access to this subroutine is desired, then the total number of yearly or daily output must be specified ( $T N W R T=1$ ), and a decision for access to Subroutine Forcut must be made (TBRM = 1), otherwise 0.0 . In the diameter class interval size ( $\mathrm{XJ1}$ ), the density of dry plant material (C9), and the bole diameter to crown diameter proportionality constant (C6) must also be specified for a given plant (JK). The year(s) of specific output desired is stored in (WRSTR) for (TNWRT) specific years. If Forcut is accessed then the year(s) of specific management are stored in (DATC'T) for (TBRM) specific years. 


\section{SECTION 2: Initialization of Variables, Diameter and Growth Eotimates for First and Second Diameter Class (Statements FMG: 18-84)}

(Statements FMG: 18-36)

If Forcut has been called for a given year (DATCT), then above ground biomass for the previous year (BBP) is ieset to the resulting volume after FORCUT management or fire simulation has occurred and one year of regrowth allowed (B1). Program control is then returned to PLANTS for another year of regrowth before Forman is executed as before.

(Statements FMG: 37-44)

The decision to cycle a plant through on a daily $(\mathrm{RGC}=3)$ or a yearly $(\mathrm{RGC}=2)$ also determines whether the plant mortality coefficient :TCR) will be made equal to (1/365) or (RSCF), for plant ( $\mathrm{JK}$ ) respectively, to be described in a later section of Forman. The biomass density estimate (T1) for a given plant (JK) which is estimated to yield tree diameters in excess of 3 " is calculated as:

$\mathrm{T} 1=(773) *(\mathrm{~V} 8) *(1-0.5 \mathrm{CCVV}) *(\mathrm{XJ} 1 / 2)$

control is transferred to PLANTS until (T1 > V5). The Forman cycle counter (MIND) is incremented by one each passage of operation to the Forman subroutine beyond the initial return statement. The change $(+,-)$ of biomass volume (G4) between current and previous year is estimated and adjusted for plant cover by the relationship:

$(\mathrm{i} 4=(0.142 * \mathrm{~B} 1 * \mathrm{~V} 8 *(1-0.5 \mathrm{CCVV}) /(\mathrm{SFAC} * \mathrm{CFAC}))-\mathrm{BBP}$.

The actual volume of the stand (V4) is estimated at $(0.142) *(\mathrm{~B} 1)$ while the adjusted volume is related to ( $\mathrm{G} 4)$ as:

$V_{5}=\left(0.142 * \mathrm{~B}^{*} \mathrm{~V}^{*} *(1-0.5 \mathrm{CCVV}) /(\mathrm{SFAC} * \mathrm{CFAC})\right.$

Finally, the minimum volume required for acceptance as a new class diameter (VTMBR) volume is estimated as:

$\mathrm{V} / 2$, for diameter class $(0-\mathrm{XJ} 1)$

$\mathrm{Z}^{*} 77.3 / \mathrm{V} 4$, for all cther diameter classes

for a given plant $(\mathrm{JK})$.

(Statements FMG: 45-70)

During the first access through Forman $(\mathrm{M}=\mathrm{MIND}=1$ ), a number of structure variables are initialized; also the volume (V2) of a plant in the initial diameter class $(0-\mathrm{XJ1})$ is estimated as:

$\mathrm{V} 2=(0.3) *(0.035 * \mathrm{~V} 8) *(2 * 2.54)^{2}$

The adjusted total volume (GTMBR) for the first diameter class is estimated as:

GTMBR $=(\mathrm{T} 1 * \operatorname{EXP}(-(1 / \mathrm{RSCF}) *(\mathrm{~V} 5-\mathrm{T} 1))$, 
and compared with the minimum volume. During the first cycling is Forman $(M=1)$ the virtual volume is used to simulate transport to the second diameter class ( $\mathrm{XJ1}=2 * \mathrm{XJ1}$ ). In this instance the volume (Z) for the mean tree in the second diameter class interval is estimated as are the number of trees (DTBR); control is returned to the main program. In subsequent cycles (M > 1) through Forman estimation of the volume in the second diameter class is performed when all other classes with the exception of the first are considered. The first diameter class $(0-\mathrm{XJ} 1)$ volume (GTMB) is estimated as described for all ( $M \geq 1)$, and $(\mathrm{G} 4)$ is used to drive the module. Finally, during all ( $M \geq 1)$ the difference between previous and current volumes of the first diameter class (DGTMB) is estimated for use in another section of Forman.

(Statements FMG: 71-84)

After setting (VTMBR = GTMBR) and determination of the number of trees (DTBR) in the first diameter class $(0-\mathrm{XJ} 1)$ for a given plant $(\mathrm{JK})$, then a number of other variables are initialized for use in the next section.

\section{SECTION 3: Tree Competition Factor, Tree Stand Characteristiciam Biomass Allocation by Diameter Class, and TRU Specific Activity Change (Statements FMG: 85- 188)}

(Statements FMG: 85-146)

Crown cover (COV), bole diameter (BAR), tree height (HGT), and diameter class competition factor (X) are all initialized on a yearly (or daily) basis for each cycle through Forman for all (N) diameter class present in the structure of plant (JK). After determination of the midpoint diameter for any diameter class (AN), the competition factor is determined in two steps:

$\mathrm{X}=(\mathrm{AN} * \mathrm{C} 6+\mathrm{V} 8)^{2}$, and $\mathrm{X}=(\mathrm{X})^{*}(\mathrm{AN})^{2} *(\mathrm{DTBR})$

where the first term represents a crown diameter factor, and the latter a bole diameter factor for a given diameter class (N) of plant (JK). In addition, crown cover (COV), tree height (HGT), and bole diameter (BAR) are determined for the mean diameter tree (AN) in each diameter class $(N)$, and standard and geometric means and deviations are estimated as (CVMN \pm BSD), respectively. Also, the crown and stern cover for each diameter class (N) are calculated as:

$\mathrm{COV}=(\mathrm{COV} * \mathrm{DTBR})$ and $\mathrm{CTRB}=\mathrm{DTBR} * \pi^{*}(\mathrm{BAR})^{2}$

after regeneration of the initial values of (COV) and (BAR).

The sum of competition factors $(\mathrm{X})$ for all diameter classes $(\mathrm{N})$ is stored in $(\mathrm{C} 8)$ as the sum of reciprocals $(\mathrm{C} 4)$; the standard and geometric mean coverage for both crown and bole for the entire stand of $(\mathrm{N})$ diameter classes is estimated as:

COVAV $=($ CVMN $) *($ TREES $) / 4047$, BAAV $=($ BMN $) *($ TREES $) / 4047$, respectively,

where (COVAV $\leq 1$ ), and (BAAV) have been redefined from previous use (Statements FMG: 137-138). Geometric means are also estimated for height (BATR), age (AGAVE), and stand TRU specific activity (ACTAV) from intermediate sums (BAAV), (AGSUM), and (ACSUM), respectively, where (TREES) represents the total number of trees of all diameter classes $(N)$ in the stand except the first plant (JK). The above ground TRU activity (TOTSA = Z3) for plant (JK) 
is used in conjunction with previous year activity (ZZ3) to estimate the change in TRU activity from one year to the next (ACTS) which is used in another section of Forman to be described.

(Statements FMG: 147-196)

For all $(\mathrm{X}>0)$ with diameter classes ( $\mathrm{L}>1)$, the mean volume tree within each class is determined by averaging minimum (AL9 - XJ1/2) and maximum (AL9 + XJ1/2) tree volumes within a given class (L). The potential amount volume (G3) allocated to a given diameter class $(\mathrm{L})$ is determined by the relationship:

$\mathrm{G} 3=(\mathrm{G} 4) *(\mathrm{X}(\mathrm{L}-1) / \mathrm{C} 8)$,

and that which determines potential intra-ciass transport is estimated by:

$\mathrm{G} 30=(\mathrm{G} 4) *(\mathrm{X}) /(\mathrm{C} 8)$.

If $(\mathrm{G} 4>0.0)$, the potential volume increment for a given diameter class volume (GTMBR) is estimated as:

$\mathrm{GTMBR}=(\mathrm{T} T \mathrm{TMR}+((\mathrm{X}(\mathrm{L}-1)-\mathrm{X}(\mathrm{L})) /(\mathrm{X}(\mathrm{L}-1)+\mathrm{X}(\mathrm{L})) * \mathrm{G} 30+\mathrm{G} 3$

If $(\mathrm{G} 4 \leq 0.0)$ the following relationship is used to estimate volume change of (GTMBR):

GTMBR $=$ GTMBR $+($ G4) * (GTMBR/V5) $*(\mathrm{C} 8-\mathrm{X}) / \mathrm{C} 8$,

for any diameter class $(\mathrm{L}>1$ ). The potential volume (GTMBR) is further adjusted for mortality losses by:

$(\mathrm{GTMBR}=\mathrm{GTMBR}-(\mathrm{CCVV} * \mathrm{GTMBR}) /(\mathrm{TCR}) *(\mathrm{C} 8-\mathrm{X} / \mathrm{C} 8)$,

where (GTMBR $\geq Z$ Z). (V6) is for the sum of all GTMBR for all volume classes. A residual volume (V4M6) is determined by difference from:

$\mathrm{V} 4 \mathrm{M} 6=\mathrm{V} 5-\mathrm{V} 6$

also $(C 7=$ VTMBR), where $(L=1)$ is initialized for summing up volumes (GTMBR) for all $(\mathrm{L} \geq$ 1). For all GTMBR $(\mathrm{L}>1)$ and $(\mathrm{C} 8>\mathrm{X}(1))$, the residual volume is added on proportional basis:

GTMBR $=$ GTMBR + V4M6 * $(1 / \mathrm{X}) / \mathrm{C} 4$, for $(\mathrm{V} 4 \mathrm{M}<0)$, or

$\mathrm{GTMBR}=\mathrm{GTMBR}+\mathrm{V} 4 \mathrm{M} 6 * \mathrm{X} /(\mathrm{C} 8-\mathrm{X}(1)),(\mathrm{V} 4 \mathrm{M} 6>0)$,

depending on the value of $(\mathrm{X})$ and (C8). The actual estimated volume is estimated as:

$\mathrm{VTMBR}=\mathrm{GTMBR} *(\mathrm{~V} 4 / \mathrm{V} 5)$,

for all diameter classes ( $L>1$ ), and the total volume sum (C7) is also estimated for use in a later section of Forman. Finally, the number of trees (DTBR) for all diameter classes $(L>1)$ is calculated as:

DTBR = VTMBR/Z; $($ VVTM = VTMBR $)$. 


\section{SECTION 4: Annual Tree Ring Estimate, Mean Age and TRU Specific Activity by Clase Diameter; Output of Stand Eotimates (Statemente FMG: 197-282)}

(Statements FMG: 197-217)

An annual running average radial growth for all trees (RINGT) is estimated by summing and averaging the product:

\section{AL9 * (VTMBR/VVTM - 1.0) $)^{1 / 2} *$ DTBR}

for all diameter classes $(\mathrm{L}>1)$.

The TRU areal activity (VA) for a given diameter class ( $\mathrm{L}>2$ ) is estimated as:

$\mathrm{VA}=(\mathrm{VTMBR} *(\mathrm{SATB})) / 0.142$

and the difference between previous and current biomass volume (VDTMB) is estimated. For any values (VDTBM, G4) (VA) is augmented in two steps as follows:

$\mathrm{VA}=\mathrm{VA}+|\mathrm{C} 4| * \mathrm{ACTS} * \mathrm{X} / \mathrm{C} 8$, and

$\mathrm{VA}=\mathrm{VA}+\mathrm{SATB} * \mathrm{VDTBM} / 0.142$

where (VA $\geq 0$ ) for a given diameter class $(\mathrm{L}>1$ ) and plant $(\mathrm{JK})$. The areal TRU activity for the first diameter class is estimated as before for all diameter classes, and for all values (DGTMB, G4), (VA) is augmented by:

$\mathrm{VA}=\mathrm{VA}+|\mathrm{G} 4|+\mathrm{ACTS} * \mathrm{X} / \mathrm{C} 8$, and

$V A=V A+S A T B * D L T M B / 0.142$,

for diameter class $(L=1)$ where $(V A \geq 0)$ for plant $(J K)$. The specific activity of the first diameter class $(\mathrm{L}=1)$, (SATB) is estimated as:

$\mathrm{SATB}=\mathrm{VA} /(\mathrm{VTMBR} / \mathbf{0 . 1 4 2})$

provided (VTMBR $>0$ ). The total areal activity (TOTAC) is initialized to equal (VA) of the first diameter class for summation with other (VA) for $(\mathrm{L}>1)$ to be described. However, if (TOTSA = 1.0) as described in Statement FMG: 216 then (TOTAC $=1.0$ ) the ratio (TOTSA/TOTAC) is used for adjusting specific activity (SATB) in all diameter classes $(\mathrm{L} \geq 1)$ to provide materials balance in estimated (SATB) to be described.

(Statement FMG: 218-228)

The age (AGTB = AI1) in which a given diameter class $(\mathrm{L} \geq 1)$ is reached for a given stand of plant (JK), and the age (AI1) in which the previous diameter class ( $L-1 \geq 1)$ is reached is used to estimate the average age of a specific diameter class $(\mathrm{L} \geq 1)$ :

$\mathrm{AATB}=(\operatorname{AATB}(\mathrm{L}-1)+\mathrm{AATB}) / 2.0$ 
provided (AGTB $>0.0$ ). If ( $A G T B=0$ ), then average age for a specific diameter class is estimated: $2^{*}(\mathrm{AATB}(\mathrm{L}-1))$. Also, the total number of years required for a diameter class $(\mathrm{L}-1)$ to reach diameter class $(L)$ is estimated by $(G A)$, where $(G A(L-1))=(A G T B-A G T B(L-1))$. If $(G A(L-1)<0)$, then it is estimated again as:

GA(L - 1) - AGTB(L - 1).

(Statements FMG: 229-236)

The specific activity (SATB) is reset to zero if (VTMBR $=0.0$ ), otherwise, if a new diameter class is created (SATB $=\mathrm{SATB}(\mathrm{L}-1)$ ) as an initial condition when (VTMBR $>0$, and SATB $=$ $0)$. The specific activity for all other classes $(L>1)$ is estimated as:

SATB $=($ SATB $) *($ TOTSA/TOTAC $)$,

where ( $S A T B \geq 0.0$ ). The latter ratio (TOTSA/TOTAC) is bounded so that division by zero is avoided when the code is operated without TRU activity present.

(Statements FMG: 237-244)

When the year(s) of desired output are reached (WRSTR $=$ AI1), then printed output is programmed for certain variables with Forman.

Output is provided here and is indicated as either daily or yearly output. If cutting and/or fire event is being simulated, then day numbers of specific managements are printed. Two groups of variables are printed (Statements FMG: 251 dind 256) under the variable names (see variable library for definition of variables).

The adjusted specific activity for all diameter classes $(\mathrm{L}>1)$ are estimated from the ratio (TOTSA/TOTAC) as described previously. Also, the mean volume (VOLTR) for trees in the stand $(L>i)$ and the total crown area for a given diameter class $(L>1)$ are estimated for output in Statements FMG: 272,273.

(Statements FMG: 276-282)

Previous year TRU activity (ZZ3) is reset to equal (Z3), and SATB for diameter classes ( $\mathrm{L} \geq 1$ ) are estimated as before if output is not required during year (AI1) or (XWRT $=0.0$ ). Finally, if Forcut management is desired (TBRM $>0$ ). then program coritrol is shifted to subroutine Forcut, otherwise, the program operation is returned to PLANTS.

SECTION 5: Input and Output Format Statements (Statements FMG: 283-299)

\section{Subroutine Forcut}

\section{SECTION 1: Array Specification, Input Requirements, and Initialization of Variables (Statements FCT: 8-35)}

All arrays in Common and those in Dirrension statements have been defined previously. The latter apply to the type and extent of management operations or fire occurrence specified. If an event(s) is desired (TBRM $\geq 1$ ), then (IBRM = TBRM) management options are entered by card input for given plant type (JK), also, for the year(s) of occurrence (DATCT = AIl) for plant (JK) 
and $($ TCUT = ITC) card input is required for removal of biomass via three diameter cut options (ACUT, BCUT, CCUT): for any event the diameter classes to be removed can be specified with a maximum of three management categories. Also, the fraction of biomass remaining within these categories is specified (RACUT, RBCUT, RCCUT) by card input. If a "Reverse-J" is to be simulated (ITC $=2$ ), then control is shifted to a later portion of Forcut to be described; $($ ITC $=3$ ) refers to a fire event.

\section{SECTION 2: Management Events, Forest Structure, Biomass, and TRU Adjustments; Output (Statements FCT: 36-125)}

Once specific diameter classes are chosen for removal or reduction (IA1, IB1, IC1, IA2, IB2, IC2) from a given stand for plant (JK), then for each category a timber reduction is performed as for the (A) category

VCUT = VTMBR * $(1-$ RACUT $) ;$

a reduction in the number of trees (DTBR) for a given diameter class $(\mathrm{K})$ is estimated as:

$\mathrm{DTBR}=\mathrm{DTBR} *(\mathrm{VTMBR}-\mathrm{VCUT}) / \mathrm{VTMBR}$.

The total biomass volume removed is determined by (VTOT) which is used for making further biomass and TRU adjustments to be described. If a fire event is simulated (ITC $=3$ ) both (VTMBR) and (GTMBR) must be adjusted:

VTMBR $=($ VTMBR - VCUT $)$ and GTMBR $=($ VTMBR $) *(V 8) *(1-$ CCVV/2)/(SFAC * CFAC).

As a result of a fire (VCUT $\leq$ VTMBR) option the TRU activity in above ground humus (Z5 = Z99) is estimated as:

$\mathrm{Z99}=\mathrm{Z99}+(0.9) *(\mathrm{VCUT}) *(\mathrm{SATB}) / 0.142$.

The TRU air activity (AA) is incremented by:

$\mathrm{AA}=\mathrm{AA}+(0.1) *(\mathrm{VCUT}) *(\mathrm{SATB}) /(0.142 * 0.30)$,

also, the above ground TRU activity for live and dead portions are estimated as:

$\mathrm{Z3}=\mathrm{Z3}-(\mathrm{SATB}) *(\mathrm{VCUT}) / 0.142 ;$

$\mathrm{Z4}=\mathrm{Z} 4+(0.2) *(\mathrm{SATB}) *(\mathrm{VCUT}) / 0.141$

Other biomass and TRU adjustments are:

$\mathrm{D} 1=(\mathrm{D} 1) *(\mathrm{C} 7-\mathrm{VTOT}) / \mathrm{C} 7$

$\mathrm{Z} 4 \mathrm{R}=(\mathrm{Z} 4 \mathrm{R})=(\mathrm{Z} 2)^{*}(\mathrm{VTOT} / \mathrm{C} 7)$

$\mathrm{Z} 2=(\mathrm{Z2})-(\mathrm{Z2}) *(\mathrm{VTOT} / \mathrm{C} 7)$ 


$$
\begin{aligned}
& \text { WND }=(\text { WND }) *(C 7-\text { VTOT }) / C 7 \\
& \text { TPGNW }=(\text { TPGNW }) *(C 7-\text { VTO } T) / C 7 \\
& \mathrm{R} 1=(\mathrm{R} 1)-(\mathrm{R} 1) *(\mathrm{VTOT} / \mathrm{C} 7) \\
& \mathrm{B} 1=(\mathrm{B} 1) *(\mathrm{C} 7-\mathrm{VTOT}) / \mathrm{C} 7
\end{aligned}
$$

Timber management for select diameter cutting (ITC $=1,2)$ have identical TRU activity and biomass adjustments with exception of above ground timber cuts (VTMBR - VCUT). They differ from a fire event by several adjustments:

$\mathrm{Z} 4=\mathrm{Z} 4+(0.25) *(\mathrm{SATB}) *(\mathrm{VCUT} / 0.141)$

and neither (Z99) or (AA) are adjusted: the Reverse-J management (ITC $=2$ ) differs from the other select diameter timber management (ITC $=1$ ) in that the former is not specified by card input. The Reverse-J option removes all diameter classes $(\mathrm{K})$ with diameters in excess of 25 inches $(\mathrm{AK}:=2 \mathrm{~K} \geq 26$ ) and operates on the remaining diameter classes with an exponential removal scheme:

$\mathrm{RVJ}=(126) *(\mathrm{EXP}(-0.197 * \mathrm{AK})) *(\mathrm{Z})$

if $\mathrm{RVJ} \leq \mathrm{VTMBR})$; VCUT $=\mathrm{VTMBR}=\mathrm{RVJ}$. If select management is simulated $(\mathrm{ITC}=1)$, then biomass volume is removed as described earlier for management via diameter selection via card input.

Output is provided in two places (Statements FCT:) with variable names as headings (see variable library for definitions of variable names). Finally, program control is returned to PLANTS.

SECTION 3: Input Format Statements (Statements FCT: 127-135)

\section{Subroutine Rmnant}

\section{SECTION 1: Array Specificantion, Input Requirements, and Initialization of Variables (Statements ANL: 2-140)}

(Statements ANL: 2-39)

All arrays have been previously defined. ${ }^{1}$ The Dimension statements apply primarily to the characteristics of herbivores modeled, and to forage characteristics of native and agricultural plants $(\mathrm{L})$. Other arrays of this type apply to population and grazing information for specific animal herds. During the initial access to RMNANT card input requirements are entered such as the mature weight ratio $(R R)$ of a given animal type of beef cattle $(R R=1.0)$, otherwise $(R R=0)$ if a certain animal is not to be included within the simulation. Other input requirements are specific for tach animal type (J); these include (P5), (N5), (AA2), and (FSUPF) which relate to herd size, grazing area, and consumption estimates (by humans) in a given region, and supplementary feed sources, respectively. Input is also required on annual grazing schedules (YRGRZ), range schedules (ONRNG), herd size for a given time interval (ANMAL), and the plants (L) included as forage (PUTZD) for animal type (J). 
Initial values of nitrogen content in forage $(\mathrm{N} 2, \mathrm{~N} 4, \mathrm{~N} 3)$ and rumen characteristics for beef cattle (M1, F1, E6) are set. Also, mature weight by age class (W), and population structure by age class (Z) are established for beef cattle, and the maximum area possible for grazing (M7) set. Other variables relating to TRU activity in or on forage (SPAC, SPAS, SPAP, SPAR, SAAP), and to amounts of available agricultural forage (HRVSP, HRVST) are also initialized.

(Statements ANL: 111-138)

If a ruminant type $(L)$ is to be considered $(R R>0)$ then mature weight $(W)$, metabolic weight $(\mathrm{M})$, and other rumen characteristics and processes $(\mathrm{R}, \mathrm{U}, \mathrm{S}$, and $\mathrm{P})$ are adjusted for this animal type (L). TRU activity in a given population group of a specific animal type (Q), TRU entering this group from all sources $(V, I>1)$, and the TRU activity in newborn $(V, I=1)$ are all initialized at zero for animal type $(J)$; the initial nitrogen content of photosynthate, standing biomass, and consumed forage for plant category (L), (PNIT, SNIT, and CNIT) are initialized to (N2) and $(\mathrm{N} 4)$, respectively, $(\mathrm{CNIT}=\mathrm{SNIT})$. Finally, the native plants and agricultural crops used as forage $(\mathrm{K})$ for animal type $(\mathrm{J})$ are summed up (SPUT) into three categories depending on the preference $(1,2,3)$ selected for this type of ruminant. The fourth preference (PUTZD $=4)$ is reserved for agricultural crops.

(Statement ANL: 139)

This statement marks the end of the initial access statement grouping described above. Subsequent access to RMNANT will shift control to statements below this value (ANL: 140)

\section{SECTION 2: Nitrogen Content Determination, Grazing Schedules, Harvest TRU Content and Biomass (Statements ANL: 141-264)}

(Statements ANL: 141-173)

If $((\mathrm{I} 1>0)$ then the utilization efficiency $(A)$ fraction of green biomass ingesteri $(G)$, the fraction of dead standing biomass (D), and the relative above ground growth rate (P4) for plant type (L) are initialized to zero. If a plant is to be utilized as forage (RGC $>0$, PUTZD $>0$, SNIT $>0$ ), then boundary conditions are set for leaf, above ground, and dead standing biomass (WND, B1, $D 1)$. The relative growth rate (D4) is estimated from the ratio $(\mathrm{B} 5(1) / \mathrm{WN} 1(\mathrm{~L}))$ where $(\mathrm{P} 4 \geq$ $-0.05)$. The total available biomass (B) is estimated from green (P2) and dry (P3) biomass estimates:

$\mathrm{P} 2=\mathrm{WND}$

$\mathrm{P} 3=(\mathrm{D} 1) *(\mathrm{WND} / \mathrm{B} 1)$

The utilization factor which adjusts the potential ingestion rate $(A)$ is calculated for each plant (L) as:

$A=(1-\operatorname{EXP}(-0.167 * B))$. 
The fraction of green biomass ingested $(G)$ by a ruminant assumes a 3:1 preference for green (or live) biomass over dead biomass ingested (D):

$$
\begin{aligned}
& \mathrm{G}=3 * \mathrm{P} 2 /(3 * \mathrm{P} 2+\mathrm{P} 3) \\
& \mathrm{D}=1-\mathrm{G}
\end{aligned}
$$

If the relative growth rate $(\mathrm{P} 4>0.142)$ then photosynthate nitrogen content (PNIT $=3.0$ ), otherwise nitjrogen content is estimated at:

$\mathrm{PNIT}=10.9 * \mathrm{P} 4+1.436$

where $(-0.05 \leq \mathrm{P} 4 \leq 0.142)$. The total amount of nitrogen in consumed green forage is estimated as a weighted average of previous nitrogen content (SNIT) and photosynthate nitrogen content (PNIT) for a plant type (L):

$\mathrm{SNIT}=(\mathrm{SNIT}) *(\mathrm{PP} 6 / \mathrm{B} 2)+(1.0-\mathrm{PP} 6 / \mathrm{P} 2) *(\mathrm{PNIT})$

if previous leaf biomass (PP6) is greater than current leaf biomass (P2), then:

$\mathrm{SNIT}=(\mathrm{SNIT}) *(\mathrm{P} 2 / \mathrm{PP} 6)+(1.0-\mathrm{P} 2 / \mathrm{PP} 6) *(\mathrm{PNIT})$

in both estimates (SNIT) is not allowed to fall below nitrogen content in dead biomass (N3). The nitrogen content of consumed forage (CNIT) accounts for preferences by ruminants for live biomass:

$\mathrm{CNIT}=\mathrm{G}^{*} \mathrm{SNIT}+\mathrm{D} * \mathrm{~N} 3$

after which $(\mathrm{PP} 6)$ is reset $(\mathrm{PP} 6=\mathrm{B} 2)$, and the water content of the ingested forage (WATER) is estimated as:

WATER $=30 *$ CNIT.

(Statements ANL*174-200)

If a specific animal herd (IN) is to be simulated ( $R R>0.0$ ), and the year number (AII) falls within a specified grazing interval (YRGRZ), then a selection is made of all harvested crops (PUTZD $=4.0$ ) for estimating the total amount of forage biomass available (HRVST) from these plants (L). When the day number (III) is one less than the harvesting date for a given crop (L):

CUTA $=$ III +1

then available supplementary forage (HRVST) is incremented by the amount removed (Q10):

$\mathrm{Q} 10=(\mathrm{B} 1-20+(20 / \mathrm{B} 1) * \mathrm{D} 1) * \mathrm{ACRES} * 4047$

HRVST $=$ HRVST + Q10 
Furthermore, the total amont of TRU activity within the forage (SPAS), and on the surface of the forage (SPAA) is estimated:

$\mathrm{SPAS}=\mathrm{SPAS}+(\mathrm{Q} 10) *(\mathrm{Z3}+\mathrm{Z} 4) /(\mathrm{B} 1+\mathrm{D} 1)$

$\mathrm{SPAA}=\mathrm{SPAA}+(\mathrm{Q} 10) * \mathrm{~A} 2 /(\mathrm{B} 1+\mathrm{D} 1)$, respectively.

Finally, a weighted average TRU activity for current year harvest (HRVST) and for the previous year (HRVSP) is estimated from a knowledge of previous year activities in and on forage (SPAP, SAAP) for a given animal group (IN) over all plants (L):

$($ SPAS $)=($ SPAS $*$ HRVST + SPAP $*$ HRVSP $) /(\text { HRVST }+ \text { HRVSP })^{2}$

$(\mathrm{SPAA})=(\mathrm{SPAA} *$ HRVST $+\mathrm{SAAP} *$ HRVSP $) /(\text { HRVST }+ \text { HRVSP })^{2}$

(Statements ANL: 201-216)

Within the population group (II), metabolic weight (M), inhalation rate (Y), blood weight (X), and the rumen capacity $(\mathrm{Cl})$ are estimated for each age group (II) within an animal type (IN). Daily forage availability (QSUM), green forage biomass (GTDR), total nitrogen content (TNIT), TRU specific activity in plants (SACT), TRU specific activity on the surface of plants (SAIR), and fraction of total diet (QFRAC) for a given plant grouping $(\mathrm{K})$ are initialized to zero. These variables apply to a grouping of plants into three preference schemes (PUTZD $=1,2,3$ ) for use by animal group (IN) which are determined by card input as described earlier.

(Statements ANL: 218-234)

The potential ingestion rate is determined from the difference between the rumen capacity (C1) and the rumen fill (S) for the (II) age group of animal type (IN):

$\mathrm{O}=(\mathrm{C} 1)-(\mathrm{S})$, where $\mathrm{O} \geq 0.001)$.

Forage plants are identified as to preference $(1 \leq$ PUTZD $\leq 3)$ and processed according to preference scheme $(K)$. The total amount of biomass available within each preference scheme for forage is estimated as:

$\mathrm{Q} 10=((\mathrm{WND}) *(\mathrm{G})+(\mathrm{D}(*(\mathrm{D} 1) *(\mathrm{WND})) /(\mathrm{B} 1) *(\mathrm{~A})$

and accumulated as:

$\mathrm{QSUM}+\mathrm{QSUM}+\mathrm{Q} 10$

In similar fashion total green biomass available in preference category $(K)$ is estimated by (GTDR):

$\mathrm{GTDR}=\mathrm{GTDR}+(\mathrm{G}) *(\mathrm{WND}) *(\mathrm{~A})$ 
and the nitrogen content factor as:

$\mathrm{TNIT}=\mathrm{TNIT}+\mathrm{CNIT} * \mathrm{Q} 10$.

Also, the TRU specific activity factor in plants of preference scheme $(\mathrm{K})$ is estimated as:

$\mathrm{S} 10=(\mathrm{WND} * \mathrm{G} * \mathrm{Z} 3+\mathrm{D} * \mathrm{D} 1 * \mathrm{WND} * \mathrm{Z} 4 / \mathrm{B} 1) * \mathrm{~A} / \mathrm{Q} 10$

and

$\mathrm{SACT}=\mathrm{SACT}+\mathrm{S} 10$.

Finally, in similar fashion, the TRU specific activity factor on plant surfaces of preference scheme $(\mathrm{K})$ is calculated as:

$\mathrm{SAO}=(\mathrm{WND} * \mathrm{G} * \mathrm{~A} 2+\mathrm{D} * \mathrm{D} 1 * \mathrm{WND} * \mathrm{~A} 2 / \mathrm{B} 1) * \mathrm{~A} / \mathrm{Q} 10$

and

$\mathrm{SAIR}=\mathrm{SAIR}+\mathrm{SAO}$

All of the factor terms are reprocessed to obtain weighted average values of (TNIT), (SACT), and (SAIR) to be described in more detail below.

(Statements ANL: 236-248)

The food preference scheme is organized into three distinct classifications: If only one preference is entered, then

$\operatorname{QFRAC}(1)=1, \operatorname{QFRAC}(2)=0.0, \operatorname{QFRAC}(3)=0.0$

If two preferences are entered, then

$\operatorname{QFRAC}(1)=0.78, \operatorname{QFRAC}(2)=0.22, \operatorname{QFRAC}(3)=0.0$.

If three preferences are entered, then

$\operatorname{QFRAC}(1)=0.7, \operatorname{QFRAC}(2)=0.20, \operatorname{QFRAC}(3)=0.10$

The total potential amount of biomass ingested from each preference category $(\mathrm{K})$ is:

$\mathrm{UP}=(\mathrm{QFRAC}) *(\mathrm{O})$

for a given preference scheme $(\mathrm{K})$. The estimated intake $(\mathrm{O})$ is thus calculated as:

$O=U P(1)+U P(2)+U P(3)$. 
The estimated weighted specific activity in (SPAC) and on forage (SPAR) for a given animal group (IN) are calculated as:

$\mathrm{SPAC}=\mathrm{SPAC}+(\mathrm{SACT}) *(\mathrm{UP}) /(\mathrm{QSUM} * \mathrm{O})$

$\mathrm{SPAR}=\mathrm{SPAR}+(\mathrm{SAIR}) *(\mathrm{UP}) /(\mathrm{QSUM} * 0)$

Also, the weighted average nitrogen content of forage $(\mathrm{N})$ consumed by animal group (IN) is estimated as:

$\mathrm{N}=\mathrm{N}+(\mathrm{UP}) *(\mathrm{TNIT}) /\left(\mathrm{QSUM}^{*} \mathrm{O}\right)$,

and the fraction of green (live) forage (GA) consumed by animal group (IN) on day (AIII) is estimated as:

$\mathrm{GA}=\mathrm{GA}+(\mathrm{UP}) *(\mathrm{GTDR}) /(\mathrm{QSUM} * \mathrm{O})$

Finally, for the first age group ( $\mathrm{IN}=1$ ) nitrogen content is set at $(\mathrm{N}=3$ ) to account for milk consumption during the weaning period.

\section{SECTION 3: Range Schedules, Rumen Function, TRU Uptake, Area Grazed (Staiements ANL: 269-376)}

(Statements ANL: 263-316)

If day number (AIII) falls within a grazing interval (ONRNG) for a selected grazing year (YRGRZ = AI1) of a given animal type (IN), then for each age group (II), within this population rumen function is simulated for that day and year. Total digestible ingested fraction (DD1) of total consumed forage for animal (IN) is:

$\mathrm{DD} 1=(1-\mathrm{GA}) *(0.45)+(\mathrm{GA}) *(0.7)$

and the total digestible energy from intake $(\mathrm{H})$ is estimated as:

$\mathrm{H}=(\mathrm{DD} 1) *(4300) *(0)$.

The microprotein fraction in the digestible fraction (M2) is set at:

$\mathrm{M} 2=0.0456,(\mathrm{~N}>1.8)$

$\mathrm{M} 2=(0.031) *(\mathrm{~N}),(0<\mathrm{N}<0.6)$

$\mathrm{M} 2=(0.023) *(\mathrm{~N}-6)+(0.0186),(0.6 \leq \mathrm{N} \leq 1.8)$

The total amount of microprotein formed from (DD1) in the rumen (H1) is estimated at:

$\mathrm{H} 2-\mathrm{H}^{*} \mathrm{M} 2$ 
The accumulation of microprotein in the rumen assuming a loss rate of ( $\mathrm{M} 1=0.52 /$ day) is: $\mathrm{P}=\mathrm{P}+\mathrm{H} 2-\mathrm{M} 1 * \mathrm{P}$,

and the ratio of microprotein content to metabolic weight for a specific age group (II) of animal type (IN) is estimated as:

$\mathrm{M} 4=\mathrm{P} / \mathrm{M}$,

where the units are $(\mathrm{g} / \mathrm{kg})$. Consequently, the fermentation digestion rate $(\mathrm{F} 2)$ is estimated as:

$\mathrm{F} 2=\mathrm{F} 1 * \mathrm{M} 4$,

where $(\mathrm{F} 1)$ is set at $(\mathrm{F} 1=0.04)$.

Hence, the total exit rate of dry matter from the rumen (E) is estimated as:

$E=(E 1+F 2)$

where

$(\mathrm{E} 6=0.25)$

and

$(\mathrm{E} \leq 0.95)$

Total potential digestibility of dry matter in the rumen (DD2) is estimated from current (DD1 ${ }^{*} \mathrm{O}$ ), and previous (U) digestible content.

$\mathrm{DD} 2=\mathrm{U}+(\mathrm{DD} 1 * \mathrm{O}) *(1-\mathrm{E}+\mathrm{F} 2 * \mathrm{E})$

Undigested food in the rumen (L1) is estimated at:

$\mathrm{L} 1=\mathrm{O}^{*}(1-\mathrm{DD} 1)+\mathrm{R}-\mathrm{U}$

where $(R)$ is the total rumen dry matter content; the total amount of food passed through the rumen undigested (L2) is estimated as:

$\mathbf{L} 2=(1-\mathrm{F} 2) *(\mathrm{E}) *(\mathrm{DD} 1) *(\mathrm{O})+\mathrm{E}+\mathrm{L} 1$.

The change in rumen dry matter content (D3) resulting from all processes is estimated as:

$\mathrm{D} 3=\mathrm{O}-\mathrm{E} * \mathrm{DD} 2-\mathrm{L} 2$,

and the change in rumen content for a given day is $R=R+D 3(R \geq 0)$, and the change in digestible rumen content is:

$\mathrm{U}=(1-\mathrm{E}) * \mathrm{DD} 2$. 
Finally, the rumen fill (S) is estimated as:

$S=(R)+(P / 1000)$

(Statements ANL: $317-376)$

The fraction of fermented energy in the form of fatty acids (X1) for a given age group (II) of animal type (IN) is estimated as:
$\mathrm{X} 1=0.747=(0.15) *(\mathrm{~N}-0.6)$
$(0.6 \leq \mathrm{N}<1.8)$
$\mathrm{X} 1=0.747$
$(0<\mathrm{N}<0.6)$
$\mathrm{X}_{1}=0.591$
$(1.8 \leq N<3)$

The total amount of energy derived from fatty acids (BB) is estimated as:

$\mathrm{BB}=(\mathrm{F} 2) *(\mathrm{H}) *(\mathrm{H} 1)$,

and energy derived from passed food $(\mathrm{E} 1)$ is calculated as:

$\mathrm{E} 1=0.25 * \mathrm{~L} 2 * 4300$.

Finally, energy derived from microprotein digestion (E2) is estimated as:

$\mathrm{E} 2=0.65 * \mathrm{P} * 4.3 * \mathrm{M} 1$.

Hence, the net available energy (E4) for potential weight gain less the amount required for respiration equals:

$\mathrm{E} 4=\mathrm{BB}+\mathrm{E} 1+\mathrm{E} 2-125 * \mathrm{M}$,

for a given age group (II) of animal type (IN); also, the required amount of supplementary feed (GSUPF) is set:

GSUPF $=(\mid$ E4 $\mid / 4.3) *($ FSUPF $) *($ HD/A55) $* Z$,

or equal to (HRVST - 1.0) if GSUPF > HRVST +1 , initially.

The weight gain (W5) of a given age group (II) is:

$\mathrm{W} 5=\mathrm{E} 4 / 1700,(\mathrm{E} 4<0, \mathrm{IN}>2)$

$\mathrm{W5}=0,(\mathrm{E} 4<0, \mathrm{IN}<2)$.

However, for $(\mathrm{E} 4>0)$, then the weight/mature weight ration (W3) is calculated as:

$\mathbf{W} 3=\mathrm{W} / \mathrm{W}(17, \mathrm{IN})$, 
where the denominator is the mature weight for animal type (IN). A weight gain adjustment factor (E5) for added maintenance requirements is estimated as:

$\mathbf{E} 5=0.0,(\mathrm{~W} 3 \geq 1.0)$

$\mathrm{E} 5=3.74+10.52 * \mathrm{~W} 3,(0.75 \leq \mathrm{W} 3<1.0)$

$\mathrm{E} 5=3.74+5.26^{*} \mathrm{~W} 3,(0.5 \leq \mathrm{W} 3 \leq 0.75)$

$\mathrm{E} 5=1.5,(\mathrm{~W} 3<0.5)$

The total weight change for a given age group (II) is estimated as:

$W=W+W 5:$

where the amounts of green (G1) and dry forage (D4) consumed by this age group are estimated at:

$G 1=(G A) *(0) *(1000)$

D4 - (1 - GA)*(O) *(1000);

and the amount of consumed forage (L2) is:

$\mathrm{C} 2=\mathrm{Ci1}+\mathrm{D} 4$.

The amount of TRU ingested with forage (C3) is estimated from specific activities in and on forage (SPAC, SPAR), total intake (C2), and the number of animal units on range (HD), those on supplementary feed (HDD), and the total number of animal units (N5 = A55) of animal type (IN):

$\mathrm{C} 3=(\mathrm{HD} / \mathrm{A} 55) *(\mathrm{C} 2) *(\mathrm{SPAC}+\mathrm{SPAR})$.

The total also includes supplementary feed TRU activity which is added to the grazing component:

$\mathrm{C} 3=\mathrm{C} 3+(\mathrm{HDD} / \mathrm{A} 55) *(\mathrm{CSUP}) *(\mathrm{SPAS}+\mathrm{SPAA})$

The total amount of supplementary feed (TOTSF) required by age group (II) of animal type (IN) on a given day (AIII) is estimated as:

TOTSF $=$ TOTSF $+($ GSUPF $) *($ HDD $) *(Z)$,

where $(\mathrm{Z})$ is the fraction of the animal herd (IN) in age categroy (II). For animals not on range which are meintained on supplementary feed, total TRU added by this herd fraction is estimated as:

$\mathrm{C} 3=\mathrm{C} 3+\mathrm{GSUPF} *(\mathrm{SPAS}+\mathrm{SPAA})$, 
where GSUPF is reset to GSUPF $=125^{*} \mathrm{M} *$ FSUPF, provided GSUPF > HRVST + 1, other wise GSUPF $=$ HRVST -1.0 ; (TOTSF) is incremented as before. Finally, the total number of square meters grazed (C4) by age group (II) for each animal unit within the category is estinıated as:

$\mathrm{C} 4=(\mathrm{C} 2) /(\mathrm{TSUMB})$,

where (TSUMB) is the total amount of green and dry standing forage available. The total number of square meters grazed (M5) by an entire age category (II) is calculated as:

M5 $=(\mathrm{C} 4) *(Z) *(\mathrm{HERD})$,

and the number of animal units/area grazed for each age category (II) is estimated as:

$\mathrm{M} 6=(\mathrm{HD}) *(\mathrm{Z}) /(\mathrm{AA} 2 * \mathrm{M} 7)$,

where (M7) is the largest total area of a region used for simulation purposes, whereas. (AA2) is the fraction of this region which is used by animal type (IN) for grazing purposes.

\section{SECTION 4: Biomass and TRU Adjustments in Forage, TRU Transport through Lung Respiration, Excretion, and Soil Conoumption (Statements ANL: 377-436)}

(Statements ANL: 377-396)

Adjustments on TRU removal from above ground biomass consisting of both within forage (Z3) and surface TRU (A2), and also from dead standing biomass (Z4) are estimated from a knowledge of total consumption from the preference categories (PUTZD) as described earlier. Total consumption of forage (Q10) is:

$\mathrm{Q} 10=(\mathrm{WND} * \mathrm{G}+\mathrm{D} * \mathrm{D} 1 * \mathrm{WND} / \mathrm{B} 1) *(\mathrm{~A})$.

and green forage consumed $(\mathrm{QGO})$ is estimated as:

$\mathrm{QGO}=-(\mathrm{G} 1) *(\mathrm{Q} 10 / \mathrm{QSUM}) *(\mathrm{UP} / \mathrm{O}) *(\mathrm{M} 6)$,

and forage consumed is:

$\mathrm{QDO}=\mathrm{D} 4) *(\mathrm{Q} 10 / \mathrm{QSUM}) *(\mathrm{UP} / \mathrm{O}) *(\mathrm{M} 6)$.

Adjustment of TRU activity in above ground biomass (Z3) is:

$\mathrm{Z3}=\mathrm{Z3}-(\mathrm{Z} 3)^{*}(\mathrm{QGG} / \mathrm{B} 1) ; \mathrm{TRU}$ in dead standing forage as:

$\mathrm{Z4}=\mathrm{Z4}-(\mathrm{Z4}) *(\mathrm{QDO} / \mathrm{D} 1),(\mathrm{Z3} \geq 0, \mathrm{Z4} \geq 0)$.

The adjustment of TRU on plant surfaces (A2) is estimated as:

$(\mathrm{A} 2)=(\mathrm{A} 2)-(\mathrm{A} 2) *(\mathrm{QGO}+\mathrm{QDO}) /(\mathrm{B} 1+\mathrm{D} 1),(\mathrm{A} 2>0)$. 
Above ground biomass (B1), and dead standing above ground biomass (D1) are adjusted:

$\mathrm{B} 1=\mathrm{B} 1-\mathrm{QGO},(\mathrm{B} 1 \geq 1.0)$,

and

$\mathrm{D} 1=\mathrm{D}) 1-\mathrm{QDO},(\mathrm{D} 1 \geq 1.0)$.

The amount of TRU in animal excretion (HUMUS) is added on to the humus activity (Z5) as:

$Z 5=(Z 5)+($ HUMUS $) *($ Q $10 /$ QSUM $) *(\mathrm{UP} / \mathrm{O})$

the hui.us compartment will be described below.

(Statements ANl: :398-414)

If a plant is of an herbaceous nature (SCTP $=1$ ), and is not a cultivated crop (PUT7D = 4), then transport of 'TRU to animal type (IN) from soil is estimated from a knowledge of consumption estimates and biomass available. The fraction of the diet (FDIET) for a given forage plant (JK) within a preference scheme (PUTZD) is estimated as:

FDIET $=$ IY/SPUT.

and the amount of soil consumed daily $(\mathrm{C} 5)$ is estimated as:

$\mathrm{C} .5=33^{*}(\mathrm{~W}) *(20) *$ FDIET/TSLMB,

where

$($ TSUMB - B1 + D1), (TSUMB $\geq 20)$

or

$\mathrm{C} 5=(3) *(\mathrm{~W}) *($ FDIET $),($ TSUMB $<20.0)$.

The TRU activity in the first soil horizon $\mathrm{Z} 1$ is adjusted:

$\mathrm{Z} 1=\mathrm{Z} 1-(\mathrm{C} 5) *(\mathrm{Z}) *(\mathrm{HERD}) *(\mathrm{Z} 1) /(\mathrm{AA} 2 * \mathrm{M} 7 * 3.0 \mathrm{E} 04)$

The amount of TRU entering the bloodstream of animal type (IN) and age category (II) is estimated as:

$\mathrm{C} 6=(\mathrm{C} 5)^{*}(0.001) *(\mathrm{Z} 1)$,

and the TRU excreted as feces is estimated at:

HUMUS $=$ HUMUS $+(0.999) *(\mathrm{C5}) *(\mathrm{Z1}) /(\mathrm{AA} 2 * \mathrm{M} 7) ;$

the total amount of HUMUS produced by an entire age category (II) is estimated after all increments have been included. 
(Statements ANL: 415-436)

The amount of TRU depositing in the pulmonary region (Q) of aninial type (IN) is estimated as:

$\mathrm{Q}=\mathrm{Q}+(0.15) *(\mathrm{~A} 1 \mathrm{TT}) *(\mathrm{Y})$,

where (A1TT) and (Y) are air concentration of TRU, and the respiration rate of animal type (IN) and age group (II). The total amount lost daily is estimated as:

$Q=Q-Q * 4.34 E-04$.

The fraction of TRU in the pulmonary regions which enters the bloodstream via the gut (L4) is estimated as:

$\mathrm{L} 4=(\mathrm{Q}) *(1.38 \mathrm{E}-04) *(0.001)$, and $($ HUMUS) is incremerted:

HUMUS $=$ HUMUS $+(0.999) *(1.33 \mathrm{E}-04) *(\mathrm{Q}) /(\mathrm{AA} 2 * \mathrm{M} 7)$.

The fraction of TRU which enters the bloodstream from the pulmonary region (L5) is estimated as:

$\mathrm{L} 5=(\mathrm{AITT}) *(3.61 \mathrm{E}-03) *(\mathrm{Q})$,

and the fraction of nonpulmonary deposits $(\mathrm{C} 7)$ which enters the bloodstream via the gut for animal type (IN) and age category (II) is estimated as:

$\mathrm{C} 7=(\mathrm{Y}) *(\mathrm{AlTT}) *(0.35) *(0.001)$.

Again (HUMUS) is incremented:

HUMUS $=$ HUMUS $+(0.999) *(\mathrm{Y}) *(\mathrm{~A} 1 \mathrm{TT}) *(0.35) /(\mathrm{AA} 2 * \mathrm{M} 7)$.

The transport of TRU to the bloodstream via forage ingestion (C8) is estimated as:

$\mathrm{C} 9=(\mathrm{C} 3) *(0.001)$,

where (C3) is the weighted average specific activity of consumed forage described earlier; (HUMUS) is incremented:

HUMUS $=$ HUMUS $+(0.999) *(\mathrm{C} 3) /(\mathrm{AA} 2 * \mathrm{M} 7)$.

Also, (HUMUS) is estimated for the entire age group (II) as:

HUMUS $=$ HUMUS * $(\mathrm{Z}) *($ HERD $)$.

The total amount of TRU entering an animal unit (IN) from all sources (V) is estimated as:

$\mathrm{V}=\mathrm{V}+\mathrm{L} 4+\mathrm{L} 5+\mathrm{C} 7+\mathrm{C} 9+\mathrm{C} 6$ 
Finally, the concentration of TRU in milk (W(11,IN) for animal type (IN) and age category (II $\geq$ 3) is estimated as:

$W(11, \mathrm{IN})=(\mathrm{W}(11, \mathrm{IN})+\mathrm{V} * \mathrm{Z} / 0.9) * 0.001 / \mathrm{X}$

where $(\mathrm{X})$ is the blood weight for animal type (IN).

\section{SECTION 5: TRU Activity in Herd, Print Options, Resetting of Variables (Statements ANL: 438-498)}

(Statements ANL: 438-444)

At the end of each salendrical year (AIII $=365$ ) each age group animal unit weight $(W)$ and TRU activity content (V) are moved into the next age class.

(Statements ANL: 445-472)

The weight of a newborn of the first age class for a given animal type (IN) is estimated as:

$\mathrm{W}(1, \mathrm{IN})=(23) *(\mathrm{RR})$

other variables are initialized for use in the following subsection.

Variable array for all $(\mathrm{L}>8)$ is allocated to storage of specific TRU and biomass estimates and averages for a given animal type $(\mathrm{K})$. Some of the variables involve some preliminary calculations (X' $\mathrm{W} 10, \mathrm{~W} 12)$ before final estimates are made:

$W 9=(Z) *(V)+W 9$

$\mathrm{W} 10=\mathrm{W} 10+(\mathrm{Z}) *(\mathrm{~V})$

$W 12=W 12+(Z) *(W)$.

They either estimate the total TRU activity $\left(\mathrm{V}^{*} \mathrm{Z}\right)$ or total biomass $\left(\mathrm{W}^{*} \mathrm{Z}\right)$ for $3-8$ year old categories.

The TRU activity/cow/breeding herd ( $3-8$ year olds) is estimated as:

$W 9=W 9 /(1-Z(1, I N)-Z(2, I N))$.

The TRU activity/cow/total herd (L-8 year olds) is:

$\mathrm{W} 10=\mathrm{W} 10+\mathrm{Z}(1, \mathrm{IN}) * \mathrm{~V}(1, \mathrm{IN})+\mathrm{Z}(2, \mathrm{IN}) * \mathrm{~V}(2, \mathrm{IN})$

The average weight/cow of the total herd is:

$W 12=W(12, I N)+Z(1, I N) * W(1, I N)+Z(2, I N)+W(2, I N)$.

The mean TRU specific activity of muscle tissue in the herd is estimated as:

$\mathrm{W} 13=(0.11086) *\left(\mathrm{~W}_{10}\right) /\left(\mathrm{W}_{12}\right) ;$ in the liver as: 
$\mathrm{W} 14=(0.45) *(\mathrm{~W} 12) /\left((0.052) *(\mathrm{~W} 12)^{0.7}\right)$

$\mathrm{W} 16=(0.45) *(\mathrm{~W} 10) /(0.075 * \mathrm{~W} 12)$.

The total amount of muscle tissue available to a human population in the region is estimated at:

$\mathrm{W} 15=(0.46) *(\mathrm{~W} 12) *(\mathrm{~N} 5) *(\mathrm{~N} 5)$,

where (N5) and (P5) have been defined previously as number of animal units, and fraction of herd consumed in the study arta, respectively. Finally, the TRU activity in newborn calves is estimated at:

$\mathrm{V}(1, \mathrm{IN})=(\mathrm{W} 9) *(0.001)$

(Statements ANL: 473-484)

Output is provided here if ( $\mathrm{AIII}=365$ and $\mathrm{II}=8$ ). Animal Biomass and Activity from all Sources are the headings and variables that will be printed.

(Statements ANL:)

Other variables are reset to current values:

SPAP $=$ SPAS, SAAP $=$ SPAA, HRVSP $=$ HRVST,

while others art initialized to zero or an appropriate value. Output follows Statement ANL: only if $(A I I=X H E R B)$ in the next section.

(Statements ANL: 488-498)

Output is provided here including input values that have not been printed previously (RR, N5, AA2, FSUPF, P5) in addition to (V)(I,IN), $W(I, I N), I=1,8$, and $W(I, I N), I=1,16$.

The concentration of TRU in milk is initialized to zero and control of RMNANT is returned to PLANTS.

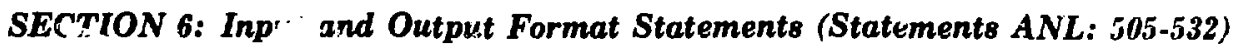

\section{E. Function Airac (SACH,YR,DAX)}

(Statements PLA: 1340-1343)

This function allows the user to substitute a function for estimating TRU air $\therefore$ : (AlRAC) using an initial concentration ( $\mathrm{SACH})$, and a relationship between such ac... the year number (YIAR), and/or day number (DAY):

AIRAC $=\mathrm{SACH}$

The relationship which is part of the program functions as a dummy $(\mathrm{SACH}=0)$ or as a continuous activity function ( $\mathrm{SACH}>0$ ). 


\section{APPENDIX H}

\section{INPUT REQUIREMENTS FTR TRU BIOLOGICAL TRANSPORT MODEL}

\section{INTRODUCTION}

This document includes input instructions for operating the TRU Biological Transport Model (BIOTRAN). In addition, a variable library (Appendix D) has been included for both input and operating variables in the program to facilitate a better understanding of model operation and the various options which can be selected for a particular run. More detailed information concerning input variables, their source, and use are included in other portions of this report.

\section{COMMENTS ON INPUT REQUIREMENTS}

The input requirements for operating the code includes a number of optional input depending on a user's needs. The code is divided into five sections:

1) Main Program

2) Function Airac

3) Forman Subroutine

4) Forcut Subroutine

5) Rmnant Subroutine

The Main Program can be accessed independently of other code components depending on user needs. All other subroutines can be accessed only in combination with the Main Program. Both Rmnant and Forman subroutines can be accessed c nly with the Main Program. However, subroutine Forcut can be accessed only if Forman is called; the function Airac can be accessed with any comkination of called subroutines. Finally, all subroutines and the Airac function can be accessed in a single run if desired. A brief description of subroutine capabilities will further clarify user options.

The Main Program contains the bulk of the transport model code, and with the exception of ruminant herd simulation, is a self-contained code. It contains all of the elements for climate simulation involving temperature, solar radiation, and precipitation. Also, all elements for simulating the growth of agricultural crops such as irrigation and harvesting schedules are included. All elements for simulating plant growth (Biomass density, $\mathrm{g} / \mathrm{m}^{2}$ ), and TRU uptake are included within this routine. Finally, the elements for simulating plant succession and interniche competition are included there.

Function Airac is used to simulate concentration of activity in air: $A=f(d, y)$, where:

$\mathrm{A}=$ activity in arbitrary units...cpm, $\mu \mathrm{Ci}$, ...etc.

d = day number

$\mathrm{y}=$ year number 
Any function of this type can be substituted in this section to tie into the Main Program.

The Forman subroutine contains the elements for determining the structure of a specific forest according to the following criteria:

1) by diameter class

2) by age group (not verified)

3) specific activity (not verified)

Specific input is required for a given type of forest. The subroutine can also be used in conjunction with grasslands and agricultural crops, although these options have not been verified or tested.

The Forcut subroutine contains all elements for forest management options based on diameter class. Any selected cutting option can be utilized including the simulation of a forest fire. The routine is interfaced with Forman and all other subroutines through the Main Program. Adjustments are made for removed activity and forest biomass as a result of the harvesting options employed.

The Rmnant subroutine contains all elements for simulating the growth of ruminant herds: beef cattle (also dairy), sheep, deer, and elk. The model utilizes biomass density estimates for native and agricultural forage crops (Main Program), and constructs a food preference and supplementary feeding scheme devised by the user. In addition to tracking TRU present in and on a given plant through selected tissues of the animal, the model also tracks inhaled TRU and TRU ingested with soil through the animal herd. Agricultural statistics on the number of animals grazing a given region, and the fraction of the area grazed by these animals during any month of the year are also available as input by the user. Output from this model consists of TRU concentrations in selected tissues for a given age group within the herd as well as averaged values such as that represented by marketed or consumed animal units. Also, the weight of each age category is available if desired by the user.

\section{COMMENTS ON PROGRAM LOADING}

Loading of the program deck depends on user requirements as noted in the input instructions. Some of the input is required for all runs independent of user needs. These include:

1) Insolation and monthly temperature characteristics for the region of interest

2) Precipitation and temperature characteristics of the site, and a reference site from which such information may be estimated

3) Edaphic and climatic features of the region in question such as elevation above sea level, frost-free period, soil holding capacity, wilting point, and field capacity

Some of this information can be obtained from field measurements, while other information can be obtained from Weather Bureau publications of the reference site. For example, Santa Fe, New Mexico, has been selected as the reference site for the study area (LASL) in question.

Some input is required for switching purposes to allow a variety of prograin capabilities as required, including which plants are to be simulated, and to allow choice of subroutines for a particular user objective. If agricultural crops are to be simulated, then a choice of irrigation information must be input if required. This includes irrigation schedules, harvests, and ariounts of water applied.

If certain plants are competitors for the same "niche," such as cold season and warm season grasses, then input information may be entered to produce weighted yield for the competitors. Input requirements in such a situation require matching of input numbers for the plants in question to be described. A run of this type can include plants which are not included in this category.

Similarly, a decision to utilize the succession module located in the Main Program is determined by card input. The succession module operates with plant types on a yearly basis in contrast to the previous module which operates on a daily basis. Generally, the latter is applied to 
perennial and annual grasses and herbs, while the succession module is applied to all perennials including grasses, shrubs, and trees. Finally, any combination of plants can be simulated with user objectives as to module use. Input options are also available for determining the fraction of the region grazed by animal herds, and for the growth of supplementary forage crops. These options are particularly important if the Rmnant subroutine is to be utilized.

Soil concentrations of TRU can be entered into the program as an option, as can soil depths, root fraction, and uptake coefficient for TRU. The model also provides for atmospheric contaminating events. One input capability is to provide measured air concentrations for a given period of time as described in the input instructions, or to substitute the Airac function with a chronic function required by the user. This instruction, however, requires program modification and caution should be used in function formulation. This function requires that either day number (1-365) or year number (or both) be included as part of the function. In addition, limits are provided for applications of such a function in terms of day and year number. Card input may also specify the year(s) of acute events as well as their duration; either or both options can be circumvented by card input.

Input specifications required for operation of the Forman subroutine if called are related to printed information or to management options if required. A user has the option of selecting up to 20 specific years of output of Forman information. The output can apply to daily as well as yearly output, depending on the type of plant simulated. Also, up to 32 different management events may be simulated through the use of Forcut subroutine as described in the input instructions on either a yearly or daily basis. Other input required for operation of this subroutine refers to plant characteristics such as the specific gravity of wood or plant bole diameter to crown diameter factor, and a relationship between plant biomass density and bole diameter for a given plant. Forman will operate on different plant types simultaneously, depending on user needs. Output from this subroutine gives stand structure by bole diameter, volume of timber by diameter class, the specific activity of the wood (averaged over total volume) for any diameter class, and the average age of a given diameter class. The latter three parameters are not verified by field observations at this time, and remain hypothetical.

Input specifications for operation of the Forcut subroutine relate specifically to timber management operations in forest ecosystems, although other plant types can be submitted for management. This subroutine allows the user a variety of management options through the simultaneous use of three plant clearing options. The user chooses diameter groups or ranges which are to be harvested or partially harvested and may simulate a heterogenous or uniform management operation. In addition, the "ser may specify a typical Forest Service "RV-J" option as a management operation in conjunction with other options if desired. Removed biomass and TRU activity are tracked back into the Main Program and into all subroutines being accessed by the user to provide for stand regeneration. A forest fire option is included as part of the operation, which integrates with local air concentrations of TRU during and after such an event: output is not provided in this subroutine.

Input into the Rmnant subroutine includes the type(s) of animal units being simulated as herds or by simulated cow-calf or sheep operations. In addition to the number of animal units being considered and the available area for grazing, other input requires a knowledge of time schedules and number of animal units grazing during any given year of activity; the fraction of harvested animal units within the region that is consumed by residents is also provided by card input. The latter is used to calculate the weighted average concentration of TRU in edible tissues of these animals in relation to total tissue consumption by region residents. Also, the fraction of supplementary feed and plants utilized by a specific animal herd are entered via card input. The user is thus allowed to enter a forage preference scheme for a specific animal unit. Output from this subroutine has been discussed. 


\section{INPUT INSTRUCTIONS FOR BIOLOGICAL TRANSPORT MODEL VIA CARD INPUT}

\section{Format for All Card Input (8F10.9) unless opecified}

\section{CARDS MUST BE IN SPECIFIED ORDER}

(required) 1 card: Insolation and Monthly Temperature Characteristics

\section{DESCRIPTION OF FIELD}

$\mathrm{T} 1=$ Minimum solar radiation, $\mathrm{Ly} /$ day received during the year

$\mathrm{T} 2$ = Maximum solar radiation, Ly/day received during the year

$\mathrm{L} 1$ = Lag period to maximum insolation, days

$\mathrm{T} 3=$ Minimum monthly daily temperature, ${ }^{\circ} \mathrm{F}$, average for site based on average daily temperature

$\mathrm{T} 4=$ Maximum monthly daily temperature, ${ }^{\circ} \mathrm{F}$, average for site based on average daily temperatures

L2 = Lag period to maximum temperature

(required) 1 card: Precipitation and Temperature Characteristics of Site

\section{DESCRIPTION OF FIELD}

TRE $=$ Total rainfall events/month totaling more than $0.1^{\prime \prime}$

TMXV = Maximurn temperature variation, on a monthly basis

$\mathrm{PRCV}=$ Coefficient of variation for precipitation/event of reference site

RELV = Elevation, feet above sea level of reference site

ALPSR $=$ Lapse rate,${ }^{\circ} \mathrm{F} / \mathrm{ft}$ from reference site

(required) 2 cards: Fraction of Precipitation Falling During a Given Month as a Function of Total Annual Precipitation

\section{DESCRIPTION OF FIELD}

$\mathrm{X}(1)=$ January fruction

$\mathrm{X}(2)=$ February fraction

$\mathrm{X}(3)=$ March fraction

$\mathrm{X}(4)=$ April fraction

$\mathrm{X}(5)$ = May fraction

$\mathrm{X}(6)=$ June fraction

$\mathrm{X}(7)=$ July fraction

$\mathrm{X}(8)$ = August fraction

$\mathrm{X}(9)=$ September fraction

$\mathrm{X}(10)=$ October fraction

$\mathrm{X}(11)=$ November fraction

$\mathrm{X}(12)=$ December fraction 
(required) 1 card: Climatic Features and Soil Characteristics

\section{DESCRIPTION OF FIELD}

$\mathrm{R}=$ Annual precipitation, inches

$\mathrm{G1}=$ Elevation of site, feet above sea level

$\mathrm{L} 3=\mathrm{Latitude}$ relative to reference site $(0.0,1.0,-1.0)$

$\mathrm{F} 1=$ Field capacity of soil, $\% \mathrm{H}_{2} \mathrm{O}$

W1 = Wilting point of soil, $\% \mathrm{H}_{2} \mathrm{O}$

$\mathrm{H}=$ Holding capacity of soil, $\% \mathrm{H}_{2} \mathrm{O}$

$\mathrm{Y} 1=$ Activity of soil, activity/gram dry weight in soil

$\mathrm{Y} 2$ = Simulation interval, years

(required) 1 card: Selectors for Subroutines and Output

\section{DESCRIPTION OF FIELD}

RSCDE $=(1.0$ or 0.0$)$ determines whether succession module is used(1) or ignored( 0$)$

RHERB $=(1.0$ or 0.0$)$ determines whether Rmnant Subroutine is called (1) or ignored(0)

RANDN = random number seed for a given run

FRMN $=(1.0$ or 0.0$)$ specifies whether Forman Subroutine is called. This value is only for output purposes. Call of Forman Subroutine is controlled by RGC

$\mathrm{ARIG}=(1.0$ or 0.0$)$ specifies whether irrigation will be part of input. This value is only for output purposes. Irrigation is specified by RIRRC(22)

$\mathrm{AICHE}=(1.0$ or 0.0$)$ specifies whether niche competition will occur. This value is only for output purposes. Niche competition is specified by RSS

FRCT $=(1.0$ or 0.0$)$ specifies whether Forcut Subroutine is called. This value is only for output purposes. Call of Forcut Subroutine is controlled by TBRM

(required) 3 cards: Selector for plants and calls of Forman Subroutine

\section{DESCRIPTION OF FIELD}

$\mathrm{RGC}(22)=$ selector for specific plants to be simulated. Plants are simulated by their position as they are listed on "Plant Number Positions for Biological Transport Model." Values for simulation are as follows:

$0.0=$ plant not simulated

$1.0=$ plant simulated, bypasses Forman Subroutine

$2.0=$ calls Forman subroutine on yearly basis

$3.0=$ calls Forman subroutine on daily basis

Yearly calls of Forman Subroutine are for structure determinations for forests and shrubs(2.0) on a yearly basis and for yerennial and annual grasses(3.0) and herbs on a daily basis.

As an example, if Pinon-Juniper and Cool Season Grasses are to be simulated, then a value of $1.0,2.0$, or 3.0 must appear in the 5 th and 18 th field positions when the cards for $\mathrm{RGC}(22)$ are 
punched. All of the rest of the fields must contain zeroes. Forman subroutine might be called on a yearly basis for Pinon-Juniper so that the value would be 2.0. For Cool Season Grasses a value of 1.0 would specify that the plant is being simulated but is not being routed through Forman.

(required) 3 cards: Irrigation

\section{DESCRIPTION OF FIELD}

RIRRC(22) = determinss whether irrigation schedule for plant type wil be part of input. Vaiues of RIRRC are as follows:

$0.0=$ no schedule

$1.0=$ card input for irrigation required

As an example, if Alfalfa were to be simulated, irrigation would be specified with a value of 1.0 in the 11th field position. All other fields would contain zeroes. Card input must follow in its position. CUTA $(22,8)$, AWATA $(22,16)$, and $\operatorname{DIRA}(22,16)$ are the input variables needed for irrigation schedules. Only one set of cards is needed per plant.

(required) 3 cards: Niche Competition

RSS $(22)=$ determines which plant groups are to be considered for niche competition for available land area. Values are as follows:

$0.0=$ bypasses niche competition module

1.0-12.0 = used to represent any plant group (same value for each) ( 2 or more plant types) which are to be adjusted fo niche competition

13-22 = values used to bypass succession module for agricultural crops

As an example, if Cool and Warm Season Grasses are to be simulated and adjusted for niche competition, a value of 3.0 might be chosen and punched in the 8 th and 18 th field positions. The value must be the same for both plants. All other fields must contain zeroes or an appropriate value.

(required) 3 cards: Acreage

\section{DESCRIPTION OF FIELD}

$\operatorname{ACRES}(22)=$ determines acreage of land associated with specific plant group. Values for acreage are as follows:

$0.0=$ assumes area of specific grid of study area

$\mathbf{x x x} . \mathbf{x x}=$ any othe value specifies acres available for growth

Example: If a specific acreage is to be associated with a plant, it must be punched in the field relative to the plant. For Alfalfa, acreage would be punched in the 11th field position of ACRES(22). 
(required) 3 cards: Soil Depth

\section{DESCRIPTION OF FIELD}

RCODE(22) = determines soil depth for specific plant types, mm. Values for soil depth are as follows:

0.0 = rooting depth via other input (SDPTH should be specified if RCODE is not utilized)

$\mathbf{x x x} . \mathbf{x x}=$ rooting depth, $\mathrm{mm}$

If a specific soil depth is simulated for Douglas Fir, for example, it would be punched in the 14th field position of RCODE(22). All other fields must contain zeroes or an appropriate value. The input value is calculated as the product of the number of soil levels desired times $250 \mathrm{~mm}$ : $\mathrm{RCODE}=5750$ for $23 \times 250 \mathrm{~mm}$ soil levels, or $24 \times 250 \mathrm{~mm}$ for some depth between 5751 to 6000 $\mathrm{mm}$.

(required) 1 card: Chronic and Contaminating Events

\section{DESCRIPTION OF FIELD}

CHRAC = initial activity in atmosphere $/ \mathrm{m}^{2}$ for chronic activity function $(0.0$ or a value greater than 0.0 required)

CYR = specifies the total number of years of an acute event $(0.0$ or value greater than 0.0 require i)

DURC $=$ duration of an acute event, days ( 0.0 or value greater than 0.0 required)

CHRSY = year of initial chronic contaminating event $(0.0$ or value greater than 0.0 required)

CHREY $=$ year of chronic contamination terminated ( 0.0 or value greater than 0.0 required)

CHRSD = day of initial acute contaminating event (day number: $1.0-365.0$ or 0.6 required)

CHRED = day of terminal contamination (day number: $1.0-365.0$ or 0.0 required)

(optional) 2 cards: Required only if CYR is greater than 0.0 , up to two cards input possible

\section{DESCRIPTION OF FIELD}

YRCNT(16) = determines specific years for application of an acute event. The years may be sequential or nonsequential, but equal to CYR. The value of CYR determines how many years are read so that if $C Y R=5$, then only one card is needed with 5 values.

(Optional) 2 cards: Required only if CYR is greater than 0.0 , up to two cards input possible

\section{DESCRIPTION OF FIELD}

DAIC (16) = determines initial day of acute event for all years involved. (1.0-365.0) The value of CYR determines how many days are read so that if $\mathrm{CYR}=8$, then only one card is needed with 8 values. 
(optional) 128 cards: Required only if CYR is greater than 0.0 and DURC is greater than 0.0 , up to 128 cards input possible

\section{DESCRIP'TION OF FIELD}

$\operatorname{AMAC}(16,64)=$ specifies amount of atmospheric activity $/ \mathrm{m}^{2}$ on a given day $(64$ day event possible) for a given year (16 separate or continuous years possible). One set of cards is necessary for each year of CYR, up to 2 cards possible. If CYR $=3$, DURC $=32$, then 4 cards are needed for each year, 12 cards in all.

(required) 1 card: Climatic Edaphic, and Subroutine Options

\section{DESCRIPTION OF FIELD}

FFP = average frost-free period of site, days

SDPTH $=$ soil depth, $\mathrm{mm}$ (equals 0.0 if input via other card input, $\mathrm{RCODE}(22)$ ) for site

UPTKE = uptake coefficient, fraction $/$ day $/ \mathrm{m} \ell$ transpiration $/ \mathrm{m}^{3}$, of TRU by plant

XPLNT = year for daily output of plant simulation

$\mathrm{XHERB}=$ controls output in Rmnant Subroutine on a daily basis. The value indicates the year(s) when such output will occur (modulus)

(optional) 88 cards: 4 cards per plant maximum

Format (8E10.3)

\section{DESCRIPTION OF FIELD}

$\mathrm{Z} 1(22,32)$ = soil activity, activity/gram dry weight in given soil horizon

(opíional) 88 cards: 4 cards per plant maximum

\section{DESCRIPTION OF FIELD}

$\mathrm{ZF}(22,32)=$ root fraction in given soil horizon

(optional) 88 cards: 4 cards per plant maximum

\section{DESCRIPTION OF FIELD}

$\mathrm{ZU}(22,32)=$ uptake coefficient for a radionuclide in a given soil horizon

(optional) 22 cards: Irrigation, Schedule required if RIRRC(22) is greater than 0.0 . One card per plant. 


\section{DESCRIPTION OF FIELD}

FORMAT (8F10.0)

CUTA $(22,8)=$ harvesting schedules, day of year. The first column is reserved for final harvesting date. All unused fields must contain zeroes.

(optional) 44 cards: Required if $\operatorname{RIRRC}(22)$ is greater than 0.0 . Two cards are needed for each plant irrigated.

\section{IJESCRIPTION OF FIELD}

AWATA $(22,16)=$ irrigation water applied, acres/inches, on specified date. All unused fields must contain zeroes.

(optional) 44 cards: Required if RIRRC(22) is greater than 0.0 . Two cards are needed for each plant irrigated.

\section{DESCRIPTION OF FIELD}

$\operatorname{DIRA}(22,16)=$ irrigation schedule, day of year, when water is applied. $\operatorname{DIRA}(\mathbf{k}, 1)$ is reserved for planting date. Two cards are needed for each plant irrigated.

\section{FORMAN SUBROUTINE}

(required) 1 card per plant

\section{DESCRIPTION OF FIELD}

TNWRT(22) = indicates how many print outs of tree structure are required

TBRM $(22)=$ indicates how many managed cuttings or events will be simulated. Maximum is 32 events (see TCUT $(22,32$ ) in FORCUT SUBROUTINE)

$\mathrm{XJ1}(11)=$ size of diameter class intervals $\left(\ldots\right.$ etc. $\left.2 "-0.2^{\prime \prime}\right)$

$\mathrm{VB}(22)=$ taper regression coefficient, relating stem diameter to above ground biomass

$\mathrm{C} 6(22)=$ crown diameter to bole diameter regression coefficient

$\mathrm{C} 9(22)=$ wood specific gravity

(required) 3 cards per plant maximum 


\section{DESCRIPTION OF FIELD}

WRSTR $(22,20)=$ stores the years (days of specific output. The number of cards read depends on the value of TNWRT. If TNWRT $=5$, then only one card is needed with only 5 values punched in the card. Filling in of zeroes is not needed.

(optional) 4 cards per plant maximum

\section{DESCRIPTION OF FIELD}

$\operatorname{DATCT}(22,32)=$ stores the years (days) of specific management generations: cutting, forest fire, etc. The number of cards read depends on the value of BRUM. If TBRM $=14,2$ cards are needed with only 14 values punched in the cards. Filling of zeroes is not needed.

\section{FORCUT SUBROUTINE}

(required) 4 cards per plant maximum

\section{DESCRIPTION OF FIELD}

TCUT $(22,32)=$ specifies the type of cut or fire event. The number of cards read depends on the value of TBRM. If TBRM $=21$, then 3 cards are needed with only 21 values punched in the cards. Filling of zeroes is not needed. TCUT is limited to 3 values:

1 = Choice Cut

2 = Reverse-J Cut

$3=$ Forest Fire

(required) 1 card per plant (for TCUT $=1,3$ )

\section{DESCRIPTION OF FIELD}

$\operatorname{ACUT}(22,1)=$ lower diameters selected for cutting " $A$ " class cut selection

$\operatorname{ACUT}(22,2)=$ upper diameters selected for cutting " $A$ " class cut selection

$\operatorname{BCUT}(22,1)=$ lower diameters selected for cutting " $B$ " class cut selection

BCUT $(22,2)=$ upper diameters selected for cutting "B" class cut selection

$\operatorname{CCUT}(22,1)=$ lower diameters selected for cutting "C" class cut selection

CCUT $(22,2)=$ upper diameters selected for cutting " $C$ " class cut selection

(required) 1 card per plant (for TCUT $=1,3$ ) 


\section{DESCRIPTION OF FIELD}

RACUT(22) = fraction of ACUT left standing

RBCUT $(22)=$ fraction of BCUT left standing

RCCUT $(22)=$ fraction of CCUT left standing

\section{RMNANT SUBROUTINE}

(required) 1 card

\section{DESCRIPTION OF FIELD}

$\mathrm{RR}(4)=$ indicates which of four animal types are simulated. Value = mature weight ratio to mature weight of beef cow, punched in col. 1-10 = beef cow, col. 11-20 = sheep, col. 21$30=$ deer, col. $31-40=$ elk. Value of 0.0 required if animal herd is not simulated.

(optional) 1 card per animal

\section{DESCRIPTION OF FIELD}

$\mathrm{P} 5(4)=$ fraction of muscle tissue and organs consumed in study area produced in that region

$\mathrm{N} 5(4)=$ number of animal units/herd

AA2(4) = fraction of region grazed by specific animal herd

FSUPF $(4)=$ fraction of supplementary feed supplied by study area

(optional) 4 cards per animal

\section{DESCRIPTION OF FIELD}

YRGRZ $(4,32)=$ Grazing intervals (years): Blank fields must be filled with zeroes: (10-20), (5090 ), etc.

(optional) 4 cards per animal unit

\section{DESCRIPTION OF FIELD}

ONRNG $(4,32)=$ days on range for day intervals $(100-290),(150-300)$, etc. Blank fields must be filled with zeroes

(optional) 4 cards per animal unit 
DESCRIPTION OF FIELD

ANMAL $(4,32)=$ number of animals grazing during each interval. Blank fields must be filled in with zeros.

(optional) 3 cards per animal unit

DESCRIPTION OF FIELD

PUTZD $(4,22)=$ plants utilized in feeding scheme of specific herbivore. Value range is from 0 to 4. A value of 0.0 implies nonutilization. A value of $1.0,2.0$, or 3.0 refers to preference of animal for given plant in descending order. Preference must be specified in the relative position of the plant. A value of 4.0 indicates plant is being used for supplementary feed. Blank fields must be filled in with zeroes. 